\title{
Spatial Nyquist Fidelity Method for Structural Models of Opto-Mechanical Systems
}

by

\section{Deborah Jane Howell}

B.S., Rensselaer Polytechnic Institute, 2001

S.M., Massachusetts Institute of Technology, 2004

Submitted to the Department of Aeronautics and Astronautics

in partial fulfillment of the requirements for the degree of

\section{Doctor of Philosophy in Aerospace Systems}

at the

\section{MASSACHUSETTS INSTITUTE OF TECHNOLOGY}

September 2007

(C) Massachusetts Institute of Technology 2007. All rights reserved.

Author

Department of Aeronautics and Astronautics

August 15, 2007

Certified by

Professor Olivier L. de Weck

Associate Professor, Department of Aeronautics and Astronautics and Engineering Systems Thesis Supervisor

Certified by

Professor David W. Miller

Professor, Department of Aeronautics and Astronautics

Thesis Co-advisor

Certified by

Professor Jeffrey Hoffman

Professor of the Practice, Department of Aeronautics and Astronautics

Certified by

George Z. Angeli, Ph.D.

Head of Systems Engineering, Thirty Meter Telescope Observatory

Accepted by

David L. Darmofal

Associate Professor of Aeronautics and Astronautics

Chair, Committee on Graduate Students 


\title{
Spatial Nyquist Fidelity Method for Structural Models of Opto-Mechanical Systems
}

\author{
by
}

\section{Deborah Jane Howell}

\author{
Submitted to the Department of Aeronautics and Astronautics \\ on August 15, 2007, in partial fulfillment of the \\ requirements for the degree of \\ Doctor of Philosophy in Aerospace Systems
}

\begin{abstract}
As technology employed in complex multidisciplinary systems such as high performance telescope systems becomes ever more sophisticated, simulation is becoming more important during the initial design phases. The method of building these simulation models is often based on engineering experience with older, potentially dissimilar systems. A method is needed to measure the fidelity of simulation models so early design decisions are made based on appropriate modeling techniques.

Previously, fidelity was a qualitative concept used to indicate a model's validity or accuracy. Here it is defined as the ability of a model to accurately predict chosen output figures of merit. In this thesis, a quantitative measure of fidelity, termed the Nyquist fidelity metric, is defined for commonly used structural model components. It expresses the ability of a finite element model to accurately predict structural eigenvalues based on the mesh size required by the spatial Nyquist criterion. The Nyquist fidelity method is developed which uses the fidelity metric to both assess the fidelity of existing complex models and to synthesize new multi-component models starting from architectural considerations such as geometric and material properties of the system. This method also estimates the error bound on the output figures of merit based on the fidelity levels and sensitivity analysis.

The Nyquist fidelity method is applied to simple sample problems to first demonstrate the methodology and it is then applied to complex telescope models to show the accuracy and computational benefits of this method compared to current methods such as model reduction. The three high performance telescope case studies are the Modular Optical Space Telescope (MOST), the Thirty Meter Telescope, and the Stratospheric Observatory for Infrared Astronomy. It is shown in the MOST example that the Nyquist fidelity method provides a $40 \%$ improvement in computational time while assuring less than 5\% modal frequency error, and less than $2.2 \%$ error in the output figure of merit.
\end{abstract}




\section{Acknowledgments}

This work was supported by the Jet Propulsion Laboratory Michelson Fellowship Program, the Association of Universities for Research in Astronomy (AURA) and the Department of Defense with SharonLeah Brown and Margaret Bryan serving as MIT fiscal officers.

This work was supervised by Prof. Olivier de Weck and Prof. David Miller. The support of my advisors, the SSL administrators, and support staff is gratefully acknowledged. A particular thanks to coworkers who provided their time and effort: The TMT modeling team of Scott Uebelhart, Soon-Jo Chung and Julien Lamamy and the MOST modeling team of Scott Uebelhart, Lucy Cohan, Elizabeth Jordan, Andrzej Stewart, Ryan Lim, and Tom Gray.

The author would also like to acknowledge the helpfulness of individuals with the SOFIA project: Prof. Dr. rer. nat. Ulrich Walter, Prof. Dr. rer. nat. Hans-Peter Röser, Eckhard Bremers, Martin Süss, Fransiska Harms, Ulrich Lampater, and Paul Keas. 


\section{Contents}

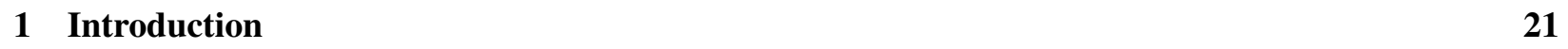

1.1 High Performance Telescopes $\ldots \ldots \ldots \ldots \ldots \ldots \ldots \ldots$

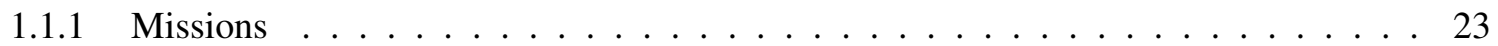

1.1 .2 Telescope design and development . . . . . . . . . . . . . . . . . . 24

1.1 .3 Role of modeling and analysis $\ldots \ldots \ldots \ldots \ldots$

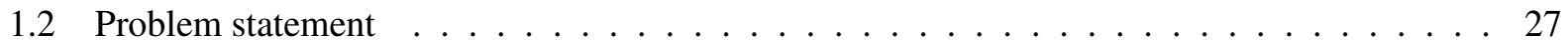

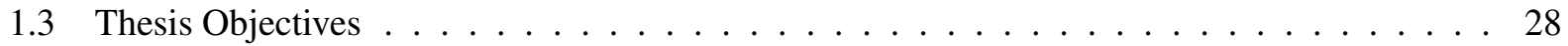

1.4 Previous Work $\ldots \ldots \ldots \ldots \ldots$

$1.4 .1 \quad$ Fidelity assessment $\ldots \ldots \ldots \ldots \ldots$. . . . . . . . . . . . . . . 29

1.4 .2 Finite element model adaptive mesh $\ldots \ldots \ldots \ldots$. . . . . . . . . 30

1.4 .3 Computationally efficient models $\ldots \ldots \ldots \ldots \ldots \ldots$

1.4 .4 Research gap analysis $\ldots \ldots \ldots \ldots \ldots \ldots$. . . . . . . . . . . . . . . . 34

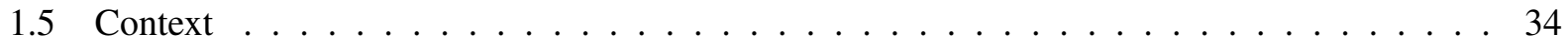

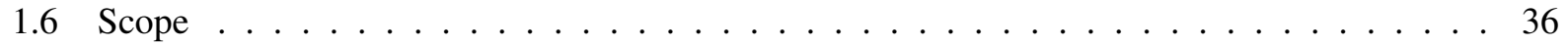

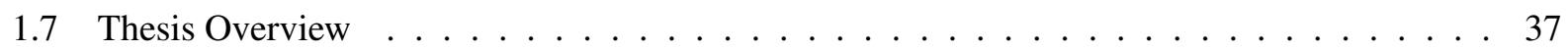

\begin{tabular}{|lll}
2 & Integrated Modeling and Analysis Framework & 39
\end{tabular}

$2.1 \quad$ Integrated Modeling . . . . . . . . . . . . . . . . . . . . . . 39

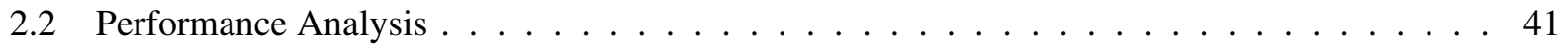

$2.2 .1 \quad$ Frequency domain analysis $\ldots \ldots \ldots \ldots \ldots \ldots$. . . . . . . . . . . 42

2.2 .2 Model reduction . . . . . . . . . . . . . . . . . . . . . . . . 44

2.2 .3 Sensitivity analysis $\ldots \ldots \ldots \ldots \ldots \ldots \ldots$

2.3 Modular Optical Space Telescope $\ldots \ldots \ldots$. . . . . . . . . . . . . . . . . . . . 48 


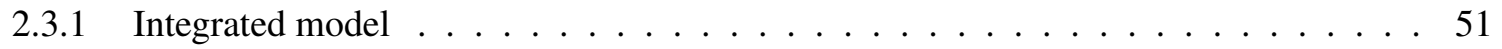

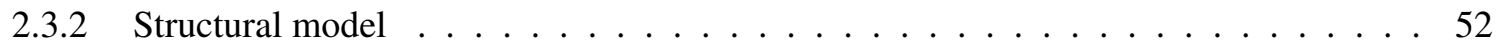

2.3 .3 Optical model . . . . . . . . . . . . . . . . . . . . 53

2.3 .4 Disturbance model . . . . . . . . . . . . . . . . . . . . . . 57

2.3 .5 Performance analysis $\ldots \ldots \ldots \ldots \ldots \ldots \ldots \ldots$

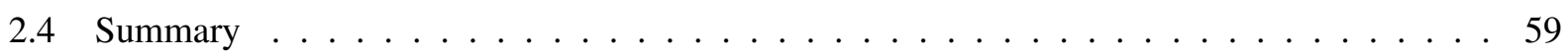

\begin{tabular}{|lll}
\hline & Nyquist Fidelity Quantification and Assessment & 63
\end{tabular}

3.1 Definitions. . . . . . . . . . . . . . . . . . . . . . . . . . 64

3.2 Fidelity Quantification using the Nyquist Theorem $\ldots \ldots \ldots \ldots$

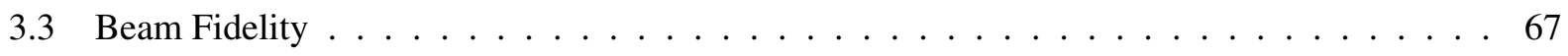

$3.3 .1 \quad$ Fidelity metric definition $\ldots \ldots \ldots \ldots \ldots \ldots$

3.3 .2 Modal frequency accuracy relationships $\ldots \ldots \ldots \ldots$

3.3 .3 Modeshape accuracy relationships $\ldots \ldots \ldots \ldots \ldots$

3.4 Plate Fidelity $\ldots \ldots \ldots \ldots \ldots$

$3.4 .1 \quad$ Circular monolithic plate . . . . . . . . . . . . . . . . . . . . . . 79

3.4 .2 General plate . . . . . . . . . . . . . . . . . . . 88

3.4 .3 Plate fidelity summary $\ldots \ldots \ldots \ldots \ldots$

3.5 Output Error Estimates $\ldots \ldots \ldots$

$3.6 \quad$ Fidelity Assessment Method $\ldots \ldots \ldots$. . . . . . . . . . . . . . . . . . 98

3.7 Fidelity Assessment of MOST Nominal Configuration $\ldots \ldots \ldots$

$3.7 .1 \quad$ Initialize model properties $\ldots \ldots \ldots$. . . . . . . . . . . . . . . . 101

3.7 .2 Critical component identification . . . . . . . . . . . . . . . . . . . . 101

3.7 .3 Fidelity metrics and modal frequency error estimation $\ldots \ldots \ldots$. . . . . 103

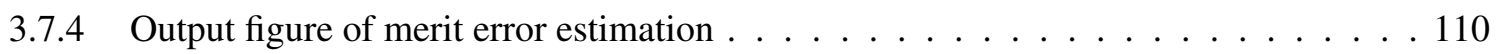

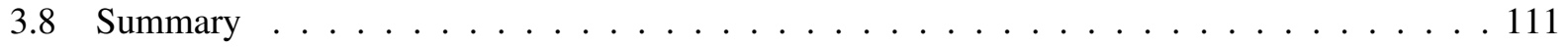

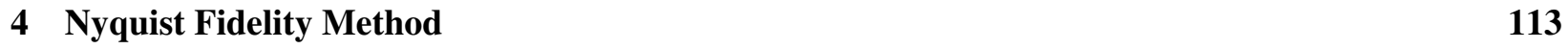

4.1 Model Synthesis Method $\ldots \ldots \ldots$. . . . . . . . . . . . . . . . . . . . . . 113

$4.2 \quad$ Beam Sample Problem $\ldots \ldots \ldots \ldots$

$4.2 .1 \quad$ Beam model synthesis using Nyquist fidelity method $\ldots \ldots \ldots \ldots$. . . . . . 117 
4.2 .2 Balanced reduction method . . . . . . . . . . . . . . . . . . . . . 123

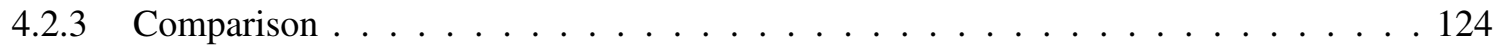

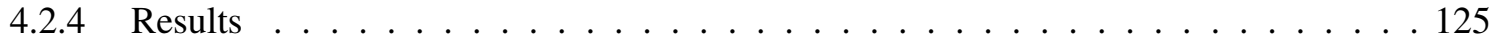

4.2 .5 Beam sample problem summary . . . . . . . . . . . . . . . . . . . . . 127

4.3 MOST Model Synthesis $\ldots \ldots \ldots \ldots \ldots$

$4.3 .1 \quad$ MOST Model Setup $\ldots \ldots \ldots \ldots$

4.3 .2 Mesh Sizing . . . . . . . . . . . . . . . . . . . . . . . . . . . . . . . 129

4.3 .3 Integrated Model and Analysis $\ldots \ldots \ldots$. . . . . . . . . . . . . 133

4.3 .4 Error Estimation . . . . . . . . . . . . . . . . . . . . . . 133

$4.3 .5 \quad$ Comparison and Results $\ldots \ldots \ldots$. . . . . . . . . . . . . . . . . . . 134

4.4 Nyquist Fidelity Method Summary . . . . . . . . . . . . . . . . . . . . . . 137

5 Application to Complex Telescopes 141

5.1 Thirty Meter Telescope . . . . . . . . . . . . . . . . . . . . . . . . . . . . . . . . . . 141

$5.1 .1 \quad$ TMT Model and Fidelity $\ldots \ldots \ldots$. . . . . . . . . . . . . . . . . . . 142

5.1 .2 TMT error estimates $\ldots \ldots \ldots \ldots \ldots \ldots$

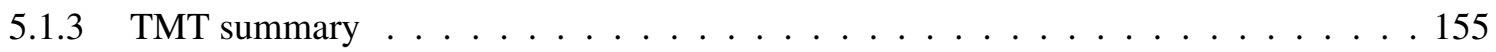

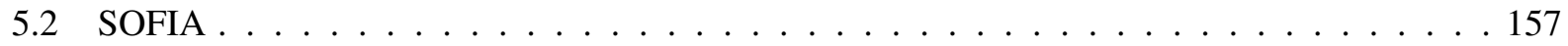

$5.2 .1 \quad$ SOFIA structural description $\ldots \ldots \ldots \ldots \ldots \ldots \ldots \ldots$

5.2 .2 Fidelity assessment $\ldots \ldots \ldots \ldots \ldots$

5.2 .3 SOFIA summary $\ldots \ldots \ldots \ldots \ldots$

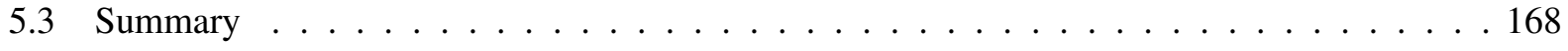

6 Conclusions and Recommendations 173

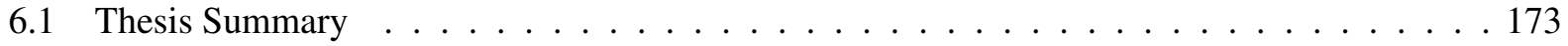

6.2 Contributions . . . . . . . . . . . . . . . . . . . . . . . 177

6.3 Best Practices for Incorporating Fidelity $\ldots \ldots \ldots \ldots$. . . . . . . . . . . . 177

6.4 Recommendations for Future Work . . . . . . . . . . . . . . . . . . . . . . . 178

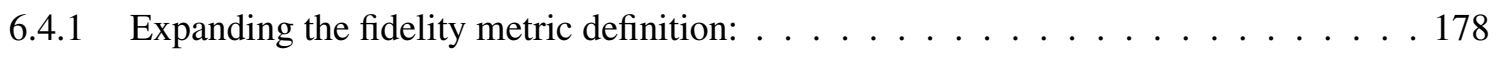

$6.4 .2 \quad$ Improving the Nyquist fidelity method $\ldots \ldots \ldots \ldots$. . . . . . . . . . 179 
\begin{tabular}{|l|l|}
\hline A Analytical Beam equations & 181
\end{tabular}

A.1 Beam Analytical Solution $\ldots \ldots \ldots \ldots$. . . . . . . . . . . . . . 181

A.2 Pinned-pinned Uniform Beam, no axial force $\ldots \ldots \ldots$. . . . . . . . . . . . . 182

A.3 Cantilever Uniform Beam $\ldots \ldots \ldots$. . . . . . . . . . . . . . . . . . . . 182

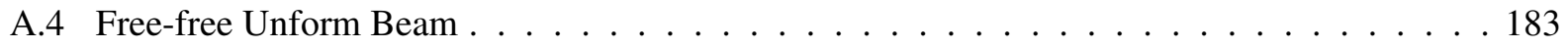

A.5 Beam Wave Number $\ldots \ldots \ldots \ldots$

\begin{tabular}{|lr}
\hline B Analytical Plate Equations & 185
\end{tabular}

B.1 Analytical Plate Equations $\ldots \ldots \ldots$. . . . . . . . . . . . . . . . . . . . . . . .

B.2 Circular Plate . . . . . . . . . . . . . . . . . . . . 186

B.3 MAC Plots for Circular Plate . . . . . . . . . . . . . . . . . . . . 187

\begin{tabular}{lr}
\hline Bibliography & 189
\end{tabular} 


\section{List of Figures}

1-1 Optical telescope assembly in Cassegrain configuration $\ldots \ldots \ldots \ldots$

1-2 Scientific telescope missions supporting the search for Earth-like planets . . . . . . . . . . 25

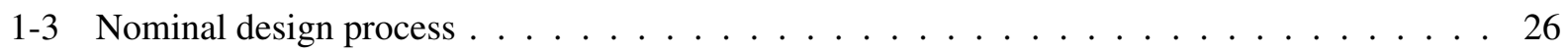

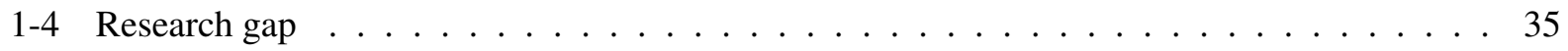

2-1 Example of an integrated model $\ldots \ldots \ldots \ldots \ldots$

2-2 Analysis process of parameterized models. Source: $[78] \ldots \ldots \ldots \ldots$

2-3 Augmented system diagram $\ldots \ldots \ldots \ldots \ldots$

$2-4$ Hankel singular values of MOST model $\ldots \ldots \ldots \ldots$

$2-5$ MOST nominal configuration $\ldots \ldots \ldots \ldots \ldots$. . . . . . . . . . . . . . 49

$2-6 \quad$ Alternate MOST configurations. Source: $[78] \ldots \ldots \ldots \ldots$

2-7 $\quad$ MOST integrated model block diagram $[78] \ldots \ldots \ldots \ldots \ldots \ldots$

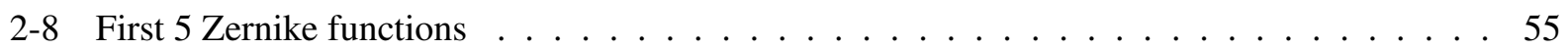

$2-9$ PSD of a RWA used on MOST $\ldots \ldots \ldots \ldots \ldots \ldots \ldots$

$2-10$ PSDs for MOST OFM $\ldots \ldots \ldots \ldots \ldots \ldots$

$3-1 \quad$ Time and spatial domain waves $\ldots \ldots \ldots \ldots \ldots$

3-2 Finite element models . . . . . . . . . . . . . . . . . . . . . . . . . . 69

3-3 Modal accuracy vs. fidelity for first 10 modes, varying properties $\ldots \ldots \ldots$. . . . . . 71

3-4 Modal accuracy vs. fidelity for first 10 modes, varying properties $\ldots \ldots \ldots$. . . . . . . 72

3-5 Power law curve fit to numerical data $\ldots \ldots \ldots \ldots \ldots$

$3-6$ Spatial aliasing in cantilever beam $\ldots \ldots \ldots \ldots \ldots$

3-7 $\quad$ Frequency folding in an 8 -element free-free beam $\ldots \ldots \ldots \ldots$. . . . . . . 77

$3-8$ MAC values for beams $\ldots \ldots \ldots \ldots \ldots \ldots$ 
3-9 $\quad$ First four circular plate modeshapes $\ldots \ldots \ldots \ldots$. . . . . . . . . . . 83

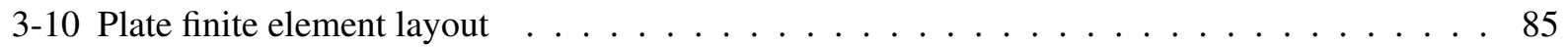

3-11 Fidelity relationships for circular plate modes $\ldots \ldots \ldots$. . . . . . . . 85

3-12 Fidelity relationships for circular plate combination modes $\ldots \ldots$. . . . . . . . 86

3-13 MAC values for two modes. Low: MAC $; 0.1$, High: MAC $i 0.9 . \ldots \ldots$. . . . . . . . 87

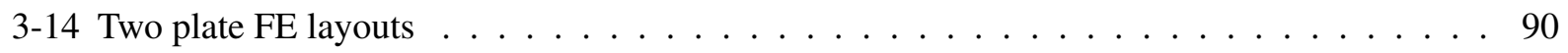

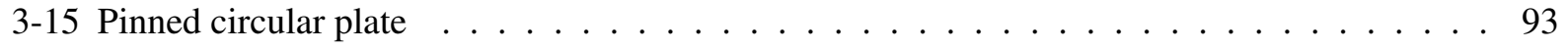

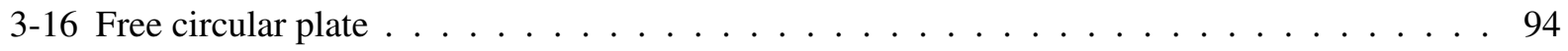

3-17 Pinned hex plate . . . . . . . . . . . . . . . . . . 95

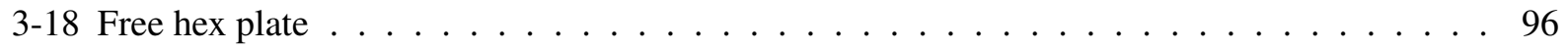

3-19 Comparison of circular and generalized plate relations $\ldots \ldots \ldots$. . . . . . . . 97

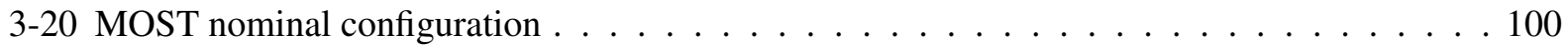

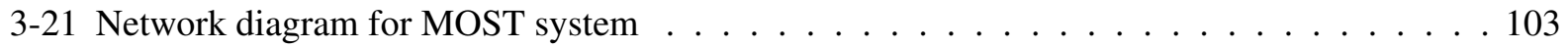

3-22 Secondary support tower of MOST $\ldots \ldots \ldots$. . . . . . . . . . . . . . . 104

$3-23$ FE meshes for plates in the MOST model $\ldots \ldots$. . . . . . . . . . . . . . . . . .

3-24 Fidelity summary for MOST components $\ldots \ldots \ldots \ldots 11$

4-1 $\quad$ Model synthesis using the Nyquist fidelity method $\ldots \ldots \ldots$. . . . . . . . . . . 115

4-2 Methods for developing structural models $\ldots \ldots \ldots \ldots$

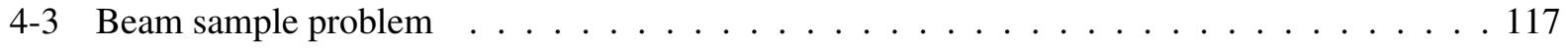

4-4 Disturbance power spectral density functions for beam sample problem . . . . . . . . . 118

$4-5$ Sensitivity of OFM with respect to the modal frequencies $\ldots \ldots \ldots$. . . . . . . . 123

$4-6$ Beam model system transfer functions $\ldots \ldots \ldots$. . . . . . . . . . . . . . 125

$4-7 \quad$ Tip displacement output PSDs $\ldots \ldots \ldots \ldots$

4-8 Calculation time comparison chart for beam sample problem . . . . . . . . . . . . . 128

$4-9$ Hankel singular values $\ldots \ldots \ldots \ldots$. . . . . . . . . . . . . . . 136

4-10 Calculation time comparison chart for MOST $\ldots \ldots \ldots \ldots$. . . . . . . . 138

4-11 Calculation time vs. percent error for MOST $\ldots \ldots \ldots \ldots$. . . . . . . . 138

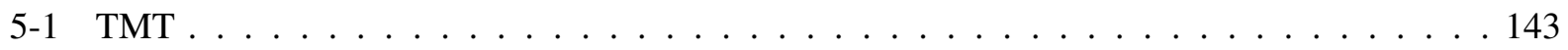

$5-2 \quad$ Integrated model diagram for TMT $\ldots \ldots \ldots \ldots \ldots$ 
$5-3 \quad$ TMT structural FEM $\ldots \ldots \ldots$. . . . . . . . . . . . . . . . . . . . . 147

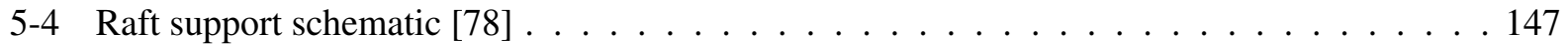

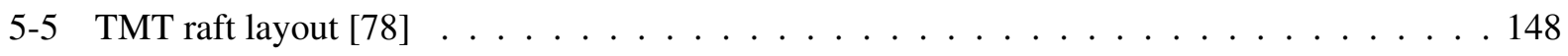

$5-6$ Wind disturbance PSDs . . . . . . . . . . . . . . . . . . . . . . . . . . . 148

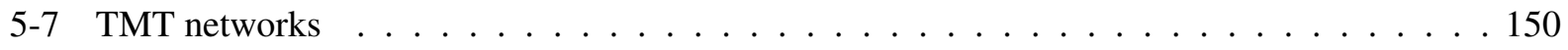

$5-8$ Quantity of bars at each fidelity level . . . . . . . . . . . . . . . . . 151

$5-9$ Quantity of lattice bars at each fidelity level $\ldots \ldots \ldots \ldots 151$

$5-10$ Four views of the lattice and elevation axis bars $\ldots \ldots \ldots$. . . . . . . . 152

5 -11 MFE vs. fidelity for TMT bars $\ldots \ldots \ldots 153$

$5-12$ Schematic of SOFIA. Source: Kärcher $[44] \ldots \ldots \ldots \ldots \ldots \ldots$

$5-13$ Schematic of SOFIA. Source: Krabbe and Kärcher $[45] \ldots \ldots \ldots \ldots \ldots$

$5-14$ Disturbance PSDs for SOFIA . . . . . . . . . . . . . . . . . . . . . . . . . . 161

$5-15$ Cumulative integrals of SOFIA disturbance PSDs $\ldots \ldots \ldots 2 \ldots \ldots$

5 -16 Network of SOFIA's structural model $\ldots \ldots$. . . . . . . . . . . . . . 163

$5-17$ SOFIA finite element model $\ldots \ldots \ldots$. . . . . . . . . . . . . . . 165

$5-18$ Quantity of plates at each fidelity level for SOFIA subsystems $\ldots \ldots \ldots 6$

$5-19$ Quantity of plates at each fidelity level . . . . . . . . . . . . . . . . 167

5 -20 Vibration Isolation System $\ldots \ldots \ldots$

$5-21$ Drive System … . . . . . . . . . . . . . . . . . . 168

$5-22$ Quantity of beams at each fidelity level $\ldots \ldots \ldots$. . . . . . . . . . . . . 169

$5-23$ Modal frequency error for SOFIA plates . . . . . . . . . . . . . . . . . . 170

5 -24 Modal frequency error for SOFIA beams . . . . . . . . . . . . . . . . . . . . . 170

B-1 MAC values for the first 10 modes of a circular monolithic free free plate . . . . . . . . 187

B-2 MAC values for the first 10 modes of a circular monolithic free free plate $\ldots \ldots$. . . 188 


\section{List of Tables}

2.1 MOST design parameters $\ldots \ldots \ldots \ldots \ldots \ldots$

2.2 Parameter values for MOST nominal model, Fig. $2-5 \mid \ldots \ldots \ldots \ldots$

2.3 OFM values for nominal MOST model, Fig. $2-5 . \ldots \ldots \ldots \ldots$. . . . . . . . 59

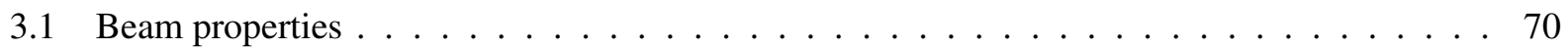

3.2 Curve fit coefficients for generalized beam relation in Eqn. $|3.8| \ldots \ldots \ldots$

3.3 MAC values for 8 -element free-free beam vs. the analytical modeshapes $\ldots \ldots \ldots$. . . . . 78

$3.4 \quad$ Number of nodal diameters and circles for a circular plate $\ldots \ldots \ldots$. . . . . . . . . . . 82

3.5 Material and geometric properties for MOST beams $\ldots \ldots \ldots 2$

3.6 Material and geometric properties for MOST plates $\ldots \ldots \ldots 2$

$3.7 \quad$ Fidelity assessment results for MOST beams $\ldots \ldots \ldots$. . . . . . . . . . . . . 105

3.8 Primary and secondary mirror sampling frequencies $\ldots \ldots \ldots$. . . . . . . 106

3.9 Estimated modal frequencies of the primary secondary and tertiary mirrors . . . . . . . . 107

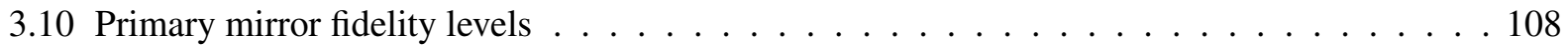

3.11 MOST plate mesh properties $\ldots \ldots \ldots \ldots$. . . . . . . . . . . . . . . . . .

3.12 Fidelity results for MOST plates $\ldots \ldots \ldots$. . . . . . . . . . . . . 109

4.1 Geometric and material properties for beam sample problem . . . . . . . . . . . 118

4.2 Sensitivity analysis values for beam sample problem with $0.1 \%$ desired modal frequency error 122

4.3 Disturbance analysis results for beam sample problem $\ldots \ldots \ldots$. . . . . . . . . . . . 124

4.4 Average calculation times for beam sample problem $\ldots \ldots \ldots$. . . . . . . . . . . 127

4.5 Mesh sizing for MOST beams for $5 \%$ and $1 \%$ MFE $\ldots \ldots \ldots$. . . . . . . . . . . 130

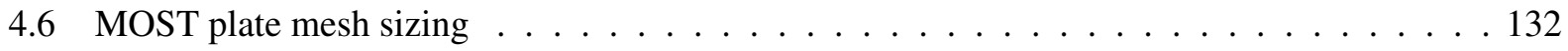

4.7 MOST mesh sizes for Nyquist fidelity method $\ldots \ldots \ldots$. . . . . . . . . 133 
$4.8 \quad$ MOST OFM values for Nyquist fidelity method $\ldots \ldots \ldots \ldots$

4.9 MOST mesh sizes for model reduction initial model and truth models $\ldots$. . . . . . . . . 135

4.10 MOST OFM values for model reduction and truth models . . . . . . . . . . . . . . . 135

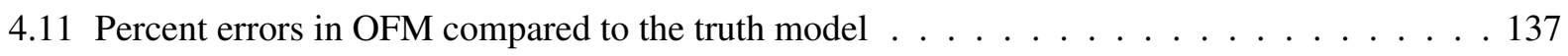

4.12 Computation times comparison for MOST $\ldots \ldots \ldots \ldots$. . . . . . . . . 137

5.1 TMT nominal design values $\ldots \ldots \ldots \ldots$. . . . . . . . . . . . . . . . . . . . . . 


\section{Nomenclature}

\begin{tabular}{ll} 
Abbreviations & \\
ACS & Attitude Control System \\
CFD & Computational fluid dynamics \\
CI & Confidence interval \\
CMS & Component Mode Synthesis \\
DS & Drive System (SOFIA) \\
FSM & Fast steering mirror \\
HSV & Hankel Singular Values \\
IM & Image motion \\
IM & Integrated model \\
IQ & Image quality \\
JWST & James Webb Space Telescope \\
LCC & Life Cycle Cost \\
LOS & Line-of-sight jitter \\
LPF & Low pass filter \\
LTI & Linear time invariant \\
MAC & Modal assurance criterion \\
MFE & Modal Frequency Error \\
MOST & Modular Optical Space Telescope \\
MS & Metering Structure (SOFIA) \\
NT & Nasmyth Tube (SOFIA) \\
OFM & Output Figure of Merit \\
OPD & Optical Pathlength Difference \\
OTA & Optical Telescope Assembly \\
PM & Primary mirror \\
PSD & Power spectral density \\
RMS & Root mean square \\
RWA & Reaction wheel assembly \\
SB & Spherical Bearing (SOFIA) \\
SIF & Scientific Instrument Flange (SOFIA) \\
SM & Secondary mirror \\
SOFIA & Stratospheric Observatory for Infrared Astronomy \\
TM & Tertiary mirror \\
TMT & Thirty Meter Telescope \\
TPF & Terrestrial Planet Finder \\
& \\
\hline
\end{tabular}


VIS

WFE

\section{Symbols}

$\delta_{S_{x}}, \delta_{S_{y}}, \delta_{S_{z}}$

$\alpha_{P_{x}}, \alpha_{P_{y}}, \alpha_{P_{z}}$

$\alpha_{S_{x}}, \alpha_{S_{y}}, \alpha_{S_{z}}$

$\alpha_{T_{x}}, \alpha_{T_{y}}, \alpha_{T_{z}}$

$\alpha_{i}$

$\delta \omega$

$\delta_{P_{x}}, \delta_{P_{y}}, \delta_{P_{z}}$

$\delta_{T_{x}}, \delta_{T_{y}}, \delta_{T_{z}}$

$\delta x$

$\delta_{i j}$

$\lambda$

$\lambda_{i}$

$\nu$

$\Omega$

$\omega$

$\omega_{k}$

$\omega_{k, \text { nom }}$

$\Phi$

$\phi$

$\phi_{i}$

$\phi_{X}$

$\rho$

$\sigma_{i}^{H}$

$\sigma_{M 1 s_{i}}$

$\sigma_{M 1 v_{i}}$

$\sigma_{M 2 s_{i}}$

$\sigma_{M S H_{i}}$

$\sigma_{z_{i}, n o m}$

$\sigma_{z_{i}}$

$\sigma_{z_{i}}^{2}$

$\Sigma_{z z}$

$\theta_{L O S}$

$\tilde{W}_{o}, \tilde{W}_{c}$

$\varpi$

$\varpi_{\theta}$

$\varpi_{r}$

$\varpi_{N y, \theta}$

$\varpi_{N y, r}$

$\varpi_{N y}$

Vibration Isolation System (SOFIA)

Wave front error

Secondary mirror displacements

Primary mirror rotations

Secondary mirror rotations

Tertiary mirror rotations

Weighting coefficient for $i^{\text {th }}$ Zernike coefficient

Percent change in frequency [\%]

Primary mirror displacements

Tertiary mirror displacements

Beam element mesh size (MOST)

Kronecker delta

Wavelength

$i^{\text {th }}$ Eigenvalue

Poisson's ratio

Modal frequency matrix

Time domain frequency [rad/sec]

Frequency of $k^{\text {th }}$ structural mode

Nominal frequency of $k^{\text {th }}$ structural mode

Modeshape matrix

True modeshape vector

$i^{\text {th }}$ Modeshape vector

Experimental modeshape vector

Volumetric mass density $\left[\mathrm{kg} / \mathrm{m}^{3}\right]$

Hankel singular values of augmented state space system

RMS value of $i^{\text {th }}$ Zernike due to the wind disturbance on the primary mirror through the dome slit (TMT)

RMS value of $i^{\text {th }}$ Zernike due to the wind disturbance on the primary mirror through the dome vents (TMT)

RMS value of $i^{\text {th }}$ Zernike due to the wind disturbance on the secondary mirror through the dome slit (TMT)

RMS value of $i^{\text {th }}$ Zernike due to the shear layer mode wind disturbance (TMT)

Nominal root mean square of output figure of merit

Root mean square of output figure of merit

Mean square of output figure of merit

State covariance matrix

LOS requirement

Balanced observability and controllability grammians

Spatial frequency $[\mathrm{rad} / \mathrm{m}]$

Spatial frequency in the azimuthal direction

Spatial frequency in the radial direction

Required spatial frequency in the azimuthal direction according to Nyquist

Required spatial frequency in the radial direction according to Nyquist

Required spatial frequency for generalized plates according to Nyquist 


\begin{tabular}{|c|c|}
\hline$\zeta$ & Modal damping ratio \\
\hline$A$ & Area $\left[\mathrm{m}^{2}\right]$ \\
\hline$A_{z d}, B_{z d}, C_{z d}, D_{z d}$ & Augmented integrated model state space matrices \\
\hline$A_{z w}, B_{z w}, C_{z w}, D_{z w}$ & Integrated model state space matrices \\
\hline$C_{L O S}$ & Optical sensitivity matrix for line-of-sight \\
\hline$D$ & Flexural rigidity $[\mathrm{Nm}]$ \\
\hline$d$ & Beam diameter $[\mathrm{m}]$ \\
\hline$d$ & White noise input \\
\hline$D_{0}$ & Nominal mirror diameter \\
\hline$E$ & Young's modulus [Pa] \\
\hline$f_{1}$ & Primary mirror focal ratio \\
\hline$F_{\lambda}$ & Fidelity metric \\
\hline$F_{\theta}$ & Plate fidelity in azimuthal direction \\
\hline$F_{r}$ & Plate fidelity in radial direction \\
\hline$G_{z w}$ & Integrated model transfer function \\
\hline$h$ & Plate thickness $[\mathrm{m}]$ \\
\hline$I$ & Area moment of inertia $\left[\mathrm{m}^{4}\right]$ \\
\hline$K$ & Stiffness matrix \\
\hline$k$ & Wavenumber \\
\hline$L$ & Beam length $[\mathrm{m}]$ \\
\hline$L_{i}$ & Lagrange multiplier matrix \\
\hline$M$ & Mass matrix \\
\hline$M$ & Secondary mirror magnification \\
\hline$n$ & Number of nodes per meter \\
\hline$n_{d}$ & Number of plate nodal diameters \\
\hline$n_{k}$ & Number of kept states \\
\hline$n_{s}$ & Total number of states \\
\hline$N_{z}$ & Number of Zernike coefficients \\
\hline$n_{\text {nodes }}$ & Number of nodes in plate model \\
\hline$n_{N y}$ & Number of nodes required by Nyquist \\
\hline$n_{\text {rings }}$ & Number of element rings in plate model \\
\hline$o$ & Number of outputs \\
\hline$p$ & Parameter variable \\
\hline$q$ & State variable for augmented integrated model \\
\hline$r$ & Radius of plate nodal circle \\
\hline$S_{w w}$ & Input (disturbance) power spectral density \\
\hline$S_{z z}$ & Output power spectral density \\
\hline$t$ & Time [seconds] \\
\hline$u$ & Input command forces vector \\
\hline$w$ & Input disturbance forces vector \\
\hline$w$ & Transverse plate displacement \\
\hline$W_{o}, W_{c}$ & Observability and controllability grammians \\
\hline$Z$ & Zernike matrix \\
\hline$z$ & Integrated model's output figure of merit \\
\hline$z_{i}$ & $i^{\text {th }}$ Zernike coefficient \\
\hline
\end{tabular}




\section{Chapter 1}

\section{Introduction}

New types of high performance telescopes operating in the optical, infrared, and other regimes are being designed to expand our knowledge about the origins of the universe and to determine whether or not life is unique on Earth [1]. These telescopes must be accurate, operable, and robust to uncertain conditions such as thermal loading, while meeting stringent performance requirements such as angular resolution, wavefront error and pointing stability. New technologies, structures and designs such as lightweight rib-stiffened mirrors, adaptive optics, and deployable structures are being implemented to meet these needs. The effect of incorporating these advancements must be thoroughly understood. Unfortunately, hardware tests can be prohibitively expensive or cannot properly simulate the operating environment. For example, to simulate the microgravity environment for a spacecraft telescope on the ground, the telescope is often suspended using wires that impart their own dynamic behavior. For both ground and airborne telescopes, wind conditions such as gusts, a major disturbance source, can be difficult to simulate in wind tunnels. Therefore, we rely ever more on simulation and modeling of the entire telescope system to give confidence that the system will meet requirements.

In this work, a quantitative fidelity metric based on the spatial Nyquist criterion is presented which ensures that known fidelity is contained in the telescope structural model for modeling analyses. The term "fidelity" is defined here as, "The ability of a simulation model to accurately predict a specific output figure of merit." The fidelity metric can be used to build a model with known fidelity from the bottom up from geometrical and material specifications, therefore more informed design decisions can be made earlier in the 
design cycle. Building a known fidelity model reduces development cost and computational time otherwise spent on a model with too much or too little fidelity. The fidelity metric can also be used to assess the fidelity of an already established structural model and refine the model if necessary. These methods will be applied to ground, air and space telescope models, each in three different phases of their design cycle.

\subsection{High Performance Telescopes}

Three different venues are being used for scientific telescope operations: ground, air and space. Since each type of telescope operates in a different environment, they are subject to different disturbances and have different advantages and disadvantages. Ground telescopes are subject to wind and seismic disturbances, and atmospheric effects degrade imaging, however instruments used on ground telescopes can be upgraded as soon as the new instrument or technology is available. Conversely, space telescopes cannot be upgraded nearly as frequently if at all, depending on orbit and resources, while space telescopes are not subject to wind or atmospheric seeing effects. The most significant disturbance onboard a spacecraft usually comes from imbalance in the reaction wheels [39]. Airborne telescopes are unique. They bypass a large portion of the atmosphere, yet are still subject to acoustic wind effects and onboard (aircraft) disturbances. They also can easily be upgraded with new instruments.

Even though these three types of telescopes operate in different environments, many have similar structural components and therefore similar modeling needs. These components consist of a primary mirror, a secondary mirror, a secondary support tower and a backstructure, called the optical telescope assembly (OTA) . The OTA is most commonly arranged in a Cassegrain or Gregorian configuration. In the Gregorian configuration, the light goes through a focus between the primary and secondary mirrors; In the Cassegrain configuration it does not. Figure 1-1 shows an OTA in the Cassegrain configuration.

Interferometers are a special class of telescopes where science light is collected at two or more apertures and the light is combined in order to increase angular resolution [16]. The individual apertures on interferometers are optical telescope assemblies, also arranged in a Cassegrain or Gregorian configuration. 


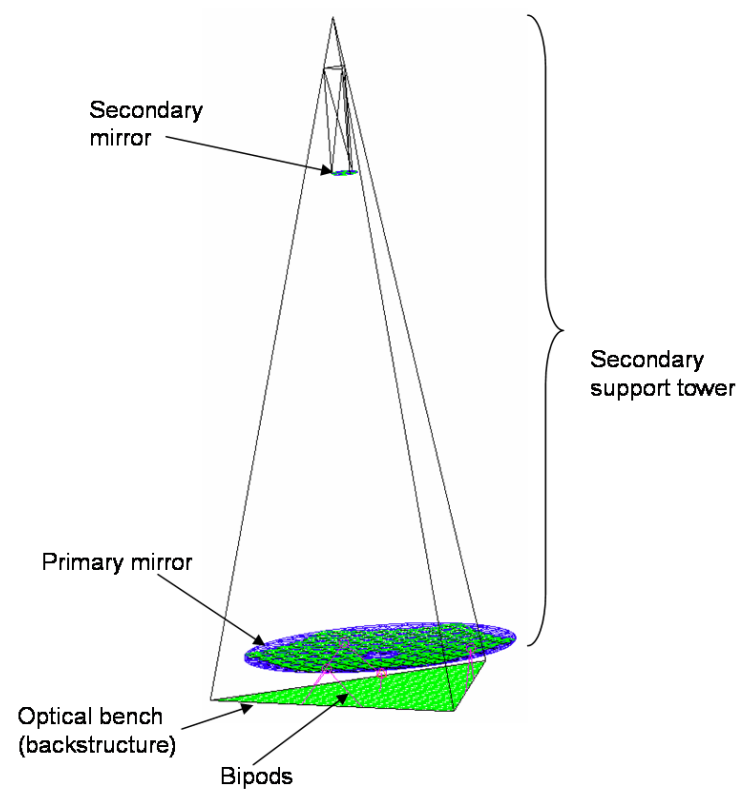

Figure 1-1. Optical telescope assembly in Cassegrain configuration

\subsubsection{Missions}

An important objective at NASA is to discover the origins of the universe and the abundance or rarity of Earth-like planets. There are many planned missions that are supporting, or will support the these objectives on the ground, in the air or in space. Two of the future spacecraft missions are the Terrestrial Planet Finder (TPF) and the James Webb Space Telescope (JWST) . There are two versions of TPF, TPF-Interferometer (TPF-I) and TPF-Coronagraph (TPF-C). TPF-I is a formation flying spacecraft infrared interferometer designed to discover planets and to investigate planet formation [47], depicted in Fig. 1-2 a). TPF-C, shown in Fig. 1-2 (b) is designed to meet the same goals, but in the visible light regime and using a coronagraph [20]. JWST, shown in Fig. 1-2 (c), is scheduled to launch in 2013 and orbit about the Sun-Earth L2 point, is a single 6.6 meter aperture infrared space telescope designed to investigate the early universe and star and planetary system creation [73].

The premier airborne telescope is the Stratospheric Observatory for Infrared Astronomy (SOFIA) [13]. SOFIA is a 2.8 meter diameter telescope mounted on a Boeing 747. The SOFIA telescope has been installed in the aircraft and is currently in the final testing stage before operation. It's optical telescope assembly is shown in Fig. 1-2 d). 
Two ground-based telescopes aiding in the search for Earth-like planets are Keck and the Thirty Meter Telescope (TMT). Keck is a set of two 10 meter diameter telescopes residing on of summit of Hawaii's Mauna Kea 85 meters apart [15] shown in Fig. 1-2 (e). The currently operational Keck operates in the visible

and infrared wavelengths. The Keck interferometer consists of the two Keck telescopes and combines the light gathered from each. TMT, shown in Fig.1-2(f), is a planned thirty meter diameter telescope operating in the ultraviolet to infrared wavelengths [54]. It is at the end of the design phase and will investigate planet, star and galaxy formation. Together with JWST, TMT will aid in the investigation of the formation of the first heavy elements and the first sources of light in the universe.

JWST, TPF-I, SOFIA, Keck, and TMT are a few examples of the current and future high-performance telescopes that are scheduled for operation in the first half of this century and will help to answer some of the unresolved questions about the universe. Despite their different roles, all incorporate the same type of optical assembly, that of a primary and secondary mirror in the Cassegrain or Gregorian configuration.

The case studies used in this thesis are the Modular Optical Space Telescope (MOST), TMT and SOFIA. MOST, currently in the conceptual design phase, is a simulation of a 3 meter diameter space telescope created by the MIT Space Systems Lab. The purpose of MOST is to investigate the trade-offs between modularity, architectural design, and telescope performance.

\subsubsection{Telescope design and development}

The design and development of high performance telescopes is a complex and multi-stage process throughout which simulation is key. NASA provides a description for the different phases in space science missions which are also followed when designing high performance telescope missions [34]. This section describes these stages and are shown in Figure 1-3. The next section details the role of modeling within the stages.

The stages defined by NASA are Pre-Phase A: Conceptual Design, Phase A: Preliminary Analysis, Phase B: Definition, Phase C/D: Design and Development, and Phase E: Operations. During Pre-Phase A, the mission needs are identified, the objectives and goals are defined and several candidate architectures are considered. In many cases engineering judgement and experience from academic, civil and private sectors drive the selection of these architectures. In Phase A, a preliminary design is selected from several candidate architectures. The instrumentation, testing plan and life cycle operations are also defined in this 


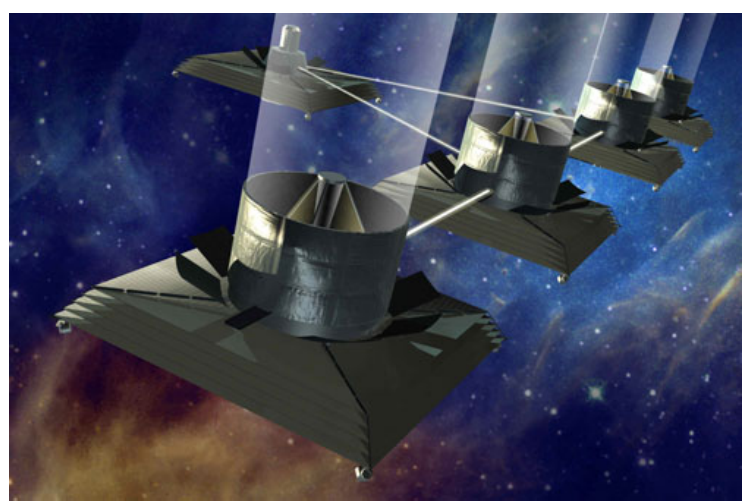

(a) TPF-I

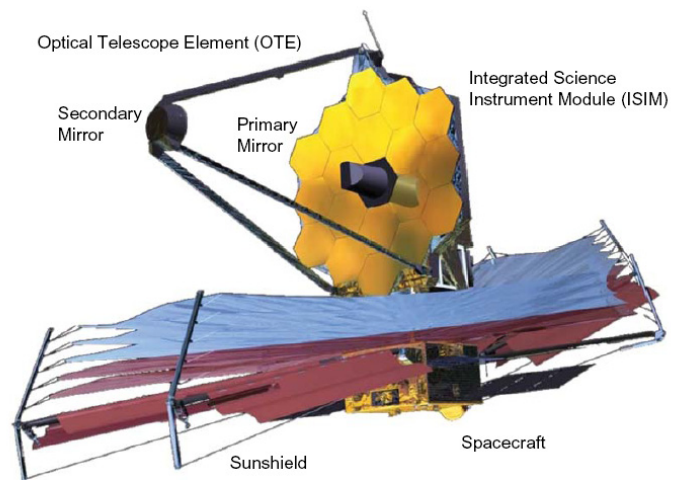

$\mathbf{J}(\mathbf{c}) \mathbf{W S T}{ }^{b}$

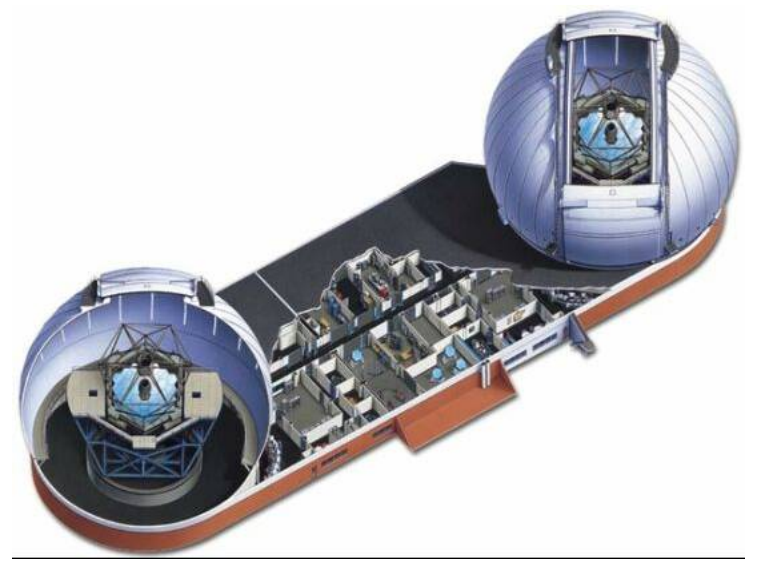

(e) $\mathrm{KECK}^{d}$

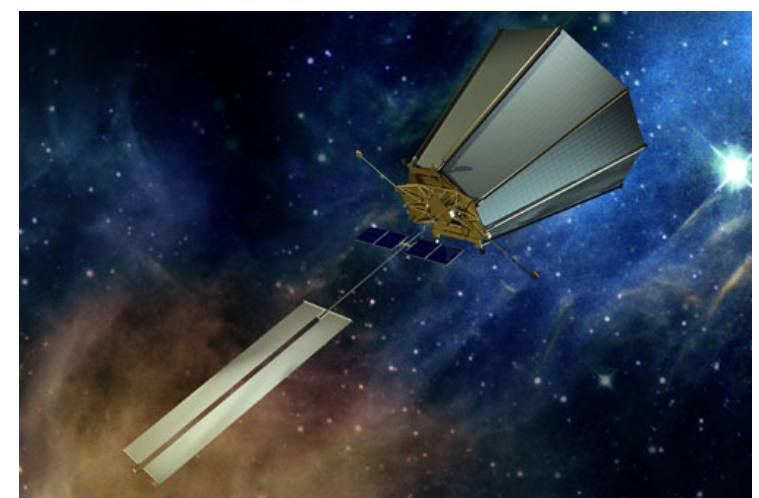

(b) TPF-C ${ }^{a}$

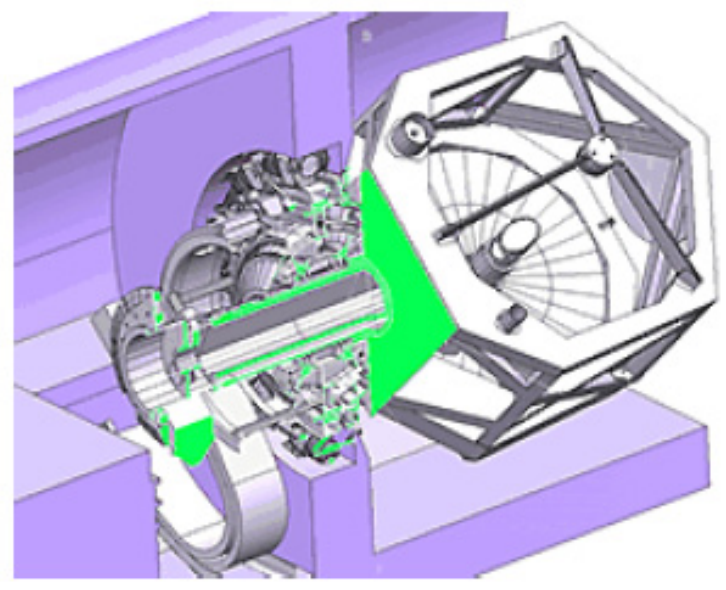

(d) SOFIA ${ }^{c}$

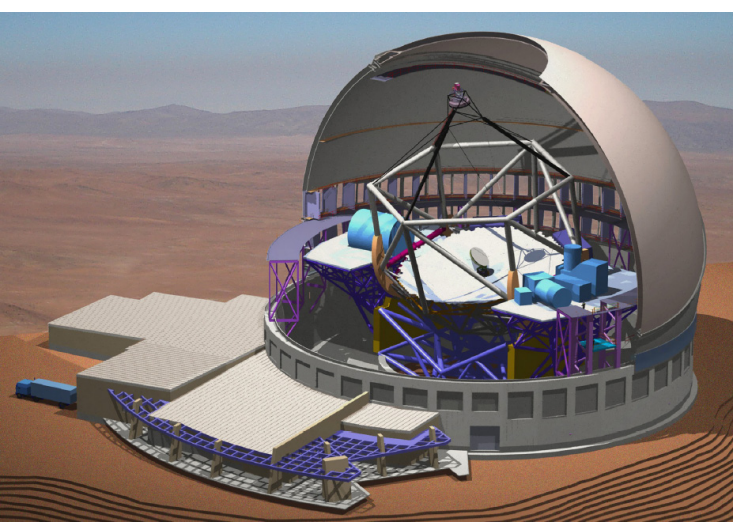

(f) $\mathbf{T M T} e^{e}$

Figure 1-2. Scientific telescope missions supporting the search for Earth-like planets

${ }^{a} \mathrm{TPF}$ images obtained from planetquest.jpl.nasa.gov.

${ }^{b}$ Image obtained from www.jwst.nasa.gov.

${ }^{c}$ Image obtained from http://www.sofia.usra.edu.

${ }^{d}$ Image obtained from www.keckobservatory.org.

${ }^{e}$ Image obtained from www.tmt.org. 


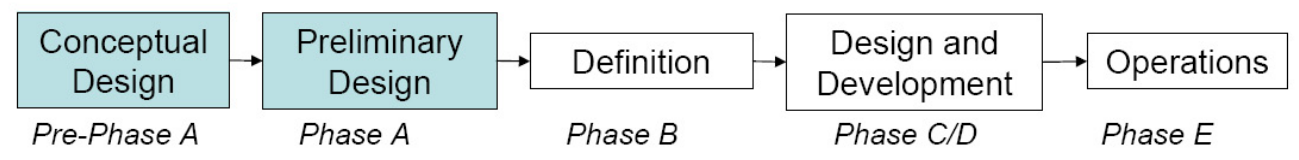

Figure 1-3. Nominal design process

phase. A baseline solution is created from the preliminary design in Phase B. Requirements, schedules, initial specifications, and the preliminary testing procedures are also carried out in Phase B. Final design, test and assembly of the flight system occurs in Phase C/D. Phase E includes the operations from launch through the time that the mission collects science data and retirement.

\subsubsection{Role of modeling and analysis}

As the design process advances, a progressively higher fidelity simulation model is usually developed to aid in design decisions. In the conceptual design phase (CDP), several candidate architectures are identified as possible solutions. Each may have different components and configurations or may incorporate new technologies. In order to produce accurate simulations that cover a wide design space, many models with differing levels of fidelity are produced. Building several "high," "low," and "mid" fidelity simulation models for each candidate design is impractical, therefore the structural simulation models created in the CDP are often low fidelity "stick" structural models based on a few chosen architectures. Some examples are the TPF-I stick model [50] and the SIM stick model [37], both used during conceptual design to explore design trades. The design decisions made in the CDP greatly impact mission life cycle cost (LCC) and performance. Unfortunately this occurs at a time when the least is known about the final design.

In preliminary analysis, a few candidate architectures are selected for further analysis and higher fidelity simulation models are constructed. During the Definition phase, simulation models are further refined. Subsystem hardware models are often available and simulation models are tested against these.

During design and development, simulation models are refined and tuned based upon the hardware tests, implicitly assuming that the hardware tests are a better predictor of operation phase behavior. The updated models are used during the operations phase to track system behavior, refine control algorithms, and predict behavior due to changes.

This thesis will concentrate on the simulation models used in the first three phases, Pre-Phase A, Phase 
A and Phase B. The reliability of simulation model results is extremely important, since they, along with hardware models, aid in the major design decisions used to create the final design. These simulation models are a major driver of design since they are exclusively relied upon in Pre-Phase A and Phase A for design decisions. Hardware models are extremely expensive and are usually confined to one configuration, therefore they are only created in Phase B, and/or C/D. In addition, hardware models can not completely simulate the operating environment, especially not in space. Simulation models can simulate the operating environment, include new technologies, and represent many different configurations and architectures. The main concern with simulation models in the absence of hardware data is whether the simulation results are valid and accurate.

The work presented in this thesis brings more sophisticated structural modeling to the conceptual and preliminary design phases where early yet crucial design decisions, a major factor in determining the total LCC, are made. It does this by providing a degree of confidence, measured by fidelity, to an otherwise un-validated simulation model.

\subsection{Problem statement}

Simulation is heavily relied upon for estimating performance of highly complex telescopes both in the conceptual and preliminary design phases. The models used must be appropriate, accurate and must have known fidelity in order to derive meaningful results. Appropriate models use mathematical representations of system components and component interfaces which behave in a similar manner as the actual system. For example, modeling a non-linear joint as a linear component is inappropriate. Accurate models produce results, such as output figures of merit, that approach the true values of the system.

The level of fidelity of these simulation models, or the ability of a simulation model to accurately predict model outputs, is often not assessed in a quantitative manner. Rather, the model is described qualitatively as being of either "low" or "high" fidelity, often progressing from lower to higher fidelity models as the candidate designs are down-selected [38]. This description of fidelity is based on engineering judgement and experience but does not ensure the accuracy of the output figure of merit (OFM). For well-known systems, this is acceptable, however unprecedented systems in the conceptual and preliminary design phases are not well-known and therefore judgement based on experience may be flawed. The resulting practice is usually 
one of two methods. First, the system is modeled using what is perceived to be a high fidelity model, then the model is reduced using a variety of methods in order to reduce computational cost. The second method applies engineering judgement from different but similar systems to create "low" and "high" fidelity models. Neither method ensures the accuracy of the output figure of merit if these methods are performed in the absence of hardware data. Furthermore, these methods can be costly in terms of computational and development time.

Design decisions made in the conceptual and preliminary design phases can have a large impact on the life cycle cost and performance, yet these decisions are made using simulation models, which in turn are largely based on engineering experience. For systems utilizing new architectures, instruments, or technologies, it is not immediately known that a system model contains a sufficient amount of fidelity to accurately predict system outputs. In order to ensure that a given model has the ability to accurately predict model outputs and to reduce computation and development time, model fidelity, which bounds the accuracy of a given output figure of merit for a given model under known disturbances, must be quantified.

\subsection{Thesis Objectives}

The main objective of this thesis is to incorporate what is known a priori about structural behavior of component models and sampling theory to create structural finite element models with known fidelity for new systems. It is assumed that the components of the finite element models are appropriate. The supporting objectives are as follows:

1. To define fidelity for component models. Using the Nyquist criterion from time-domain sampling theory, the ability of a given finite element mesh to represent spatial waveforms present in the structural model can be assessed. The component models examined here are beams and plates, two commonly used finite elements during the conceptual and preliminary design phases.

2. To define a relationship between fidelity and the accuracy of a given OFM. Several component models with different geometries, material properties, boundary conditions and mesh densities are created, and the change in the accuracy of the modal frequency estimate with respect to the change in fidelity is observed. To be useful in future projects, this relationship is generalized for beams and 
plates of different sizes and compositions.

3. To develop and demonstrate methods for applying the fidelity metric to a system model. Two methods of applying the fidelity metric are developed here. First, a method to create a model with known fidelity from geometric and material specifications is created. Second, a method which enables the user to gauge the fidelity of an existing model is created.

4. To demonstrate the usefulness of fidelity methods in numerical examples. A spacecraft telescope system is modeled using the Nyquist fidelity method and the current model reduction method and a comparison of accuracy and computation time is made. Also, the fidelity of three real-world telescope models is assessed.

\subsection{Previous Work}

This section contains a review of literature relevant to fidelity assessment, finite element adaptive mesh, and computationally efficient models. It also describes the context of fidelity analysis within the design process, integrated modeling and other types of analyses.

\subsubsection{Fidelity assessment}

Johsi, Melody and Neat, 1997 [43] compare the optical pathlength difference (OPD) predicted by low-, mid-, and high-fidelity integrated structural/optical models of a complex opto-mechanical system to experimental measurements of OPD. Engineering experience is used to assign each fidelity level. They demonstrate that the fidelity level is related to frequency response range; The low fidelity model predicts the low frequency component of the OPD well, the mid-fidelity model predicts mid-frequency ranges well. They also note, that whereas the high fidelity model is the best predictor, it also comes at a high computational cost. An important inference that can be made from this paper is that mesh density is related to accuracy of the model in specific frequency ranges.

In engineering analysis studies, fidelity has been assessed in qualitative terms, usually based on engineering judgement. Guruswamy, 2002 [38] presents the results of a survey given to aerospace industry experts who were asked to rank modeling methods in terms of complexity resulting in a fidelity index. The 
terms "complexity" and "fidelity" are used interchangeably in this paper. The methods are ranked from high to low fidelity in four different disciplines: fluids, structures, controls and propulsion. These rankings are a first cut attempt at fidelity quantification, yet they are described in qualitative terms.

The Structural Vibration Modeling and Verification Technology Exchange Forum [2] (SVMV) was a workshop convened in 2005 in which industry experts presented best practices for structural vibration modeling methods. A heuristic, relating to the fidelity presented in this work, was presented which suggested a finite element density of two nodes per spatial wavelength. However a formal measure of the fidelity was not presented.

In 2006, NASA published NASA-STD-[I]-7009 [67] whose purpose is to "ensure that the credibility of the results from models and simulations $(M \& S)$ is properly conveyed to those making critical decisions" and to "assure (sic) that the credibility of the results from M\&S meets the project requirements." A Summary Credibility Scale is presented which describes to decision makers the appropriateness of a model based on accuracy, uncertainty, the scope of its operations, etc. The scale has four levels of increasing credibility, 1 - Research 2 - Development 3 - Production 4 - Rigorous. Each level is described in qualitative terms. In the future, NASA plans to release discipline-specific credibility standards which may or may not include quantifiable metrics. The study of fidelity presented in this thesis is related to the credibility scale since both aim to provide important information to decision makers. However, in this thesis, the appropriateness of the model is measured quantitatively using fidelity metrics.

In the literature for fidelity assessment, fidelity has been described qualitatively. This thesis attempts to quantify fidelity.

\subsubsection{Finite element model adaptive mesh}

Adaptive meshing methods for finite element (FE) models is a rich field. Error estimates on the finite element model are used to refine a finite element mesh, and can be used in adaptive meshing programs, where the mesh is refined based on loading profiles. Bathe [11], Hughes [41], and Babuska [10] provide texts that introduce the FE method and address error estimates and bounding. Numerous studies have been performed which attempt to estimate the error of a given finite element mesh. Since this thesis addresses the dynamic analysis of structurally complex systems, a few references relevant to mesh densities of these FE models is 
presented.

Bathe, 1990 [12] describes the concept of hierarchical finite element models, a concept similar to fidelity. In this paper, it is emphasized that the simulation model is only a mathematical representation of the actual system. The mathematical representation cannot represent the physical system exactly; therefore the behavior of the physical system cannot be predicted exactly. One can only hope to gain insight into the physical problem, and therefore the selection of the mathematical model is key. In finite element analysis, the model can be refined by increasing the mesh density or by using a higher-order mathematical representation of the element. A set of successively refined models are called hierarchical models. Bathe compares the accuracy of the hierarchical models to a "very comprehensive mathematical model," in order to demonstrate how new behaviors and increased accuracy are gained as the model is refined. An important insight from this work is that by increasing the mesh density of the model, a model more closely matching the mathematical model is created.

In a comprehensive study, Grätsch and Bathe, 2004 [36] describe techniques for finite element a posteriori error estimation due to discretization error. Assuming that an appropriate mathematical model has been chosen, error estimates based on the global energy norm are presented. The major problem cited is that accurate error estimates are obtained at the computational cost of running a much higher fidelity model. Conversely, error estimates that have low computational cost also have no provable mathematical bounds.

Cook and Avrashi, 1992 [21] attempt to estimate finite element model errors specifically for dynamic models, such as the models used in the case studies in this thesis. Using the strain energy distributions, the error in the natural frequency calculated by the finite element model is estimated. An optimal mesh is produced by repositioning the nodes or remeshing the model. There is an optimal mesh calculated for each natural mode. The method described in this thesis provides a mesh which is accurate to a certain level for all frequencies up to a user-specified upper frequency.

Transient loading problems have been approached using direct integration methods and modal superposition analysis. Wiberg, 1994 [82] uses a posteriori error estimates to adapt both the time step and spatial mesh using the direct integration method. This is subject to interpolation errors between meshes and is a computationally expensive method since the mesh and time step can be changed at each integration approximation iteration. Dutta, 1999 [29] presents an adaptive mesh method for transient loading problems using 
modal superposition analysis. Modal superposition analysis is suitable for long time domain, structurally complex systems. Indeed, the loading profiles addressed in this thesis are considered to be long duration stochastic excitations with a known frequency content.

Many of the adaptive meshing methods discussed above are appropriate for models in the detailed design phase since in this phase one or a few designs are analyzed. The work presented in this thesis addresses the dynamic analysis of structurally complex systems, such as space telescopes in the conceptual design phase where any configurations and architectures are considered. Therefore, this thesis provides an alternative method of building finite element models to existing adaptive mesh schemes which is more focused on the conceptual design phase.

\subsubsection{Computationally efficient models}

The trade-off between model fidelity and computation time is the impetus for the creation of many alternative modeling methods which aim to minimize the computational cost while retaining accurate results. Like adaptive mesh, this is a very rich field. Since this thesis presents an alternative modeling method for conceptual design telescope systems, the method's computational efficiency is analyzed. Here, three types of modeling methods that are designed to reduce computational time are discussed.

Model reduction is a common modeling practice in which a computationally expensive, high fidelity and high complexity model is transformed into a computationally efficient model which retains the important parts of the high fidelity model, thereby retaining a level of accuracy. There are several types of model reduction methods, often tailored to specific problems of interest such as large order multidisciplinary problems, real-time applications in which computation time must be short and circuit design. Current state of the art model reduction methods include balanced truncation [66] [64](Antoulas and Sorenson [8] provide a survey), proper orthogonal decomposition (Lumley [51]), and Krylov methods (Gallivan [31]). In recent years, model reduction as been extended to large scale systems. Willcox and Peraire, 2002 [83] combine two popular methods, balanced order reduction (truncation) and proper orthogonal decomposition, in order to address extremely large order models (e.g. models with tens of thousands of states), such as those in computational fluid dynamics. Lieu et al, 2006 [49] demonstrate how model reduction can be applied to a multidisciplinary model of an entire aircraft. 
Surrogate based optimization methods are targeted towards system models requiring long computational times. Prominent among the specific problems, are computational fluid dynamics (CFD) models in which several models with slightly different designs are analyzed serially in the time domain in order to find an optimal design based on one or more performance criteria. This is called surrogate based optimization (SBO) and it is made computationally efficient by the use of surrogate models in combination with highfidelity models. Eldred, 2004 [30] divides the surrogate models into three categories, data-fits to highfidelity model points, reduced-order models and models with variable fidelity/complexity. Queipo 2005 [71] provides a survey of the numerous SBO methods that have been developed; The following are a few of these methods which specifically address high and low fidelity models. Chang 1993 [18] develops a method which updates a coarsely meshed low fidelity structural finite element model with a finely meshed model using a sensitivity based scaling factor. The updated low-fidelity model provides sufficient accuracy to be appropriate for optimization analysis. Zadeh, 2002 [84] similarly updates the low-fidelity model, but uses response-surface modeling to do so. Alexandrov, 2001 [4] describes a management framework which uses the low fidelity model for a large portion of the optimization iterations, and uses the high-fidelity model occasionally to correct the optimization direction. Robinson [72] builds on this by mapping the design vector of the high-fidelity models to the design vector of the low fidelity models, thereby enabling models of different complexities and with different parameterizations to be used in SBO methods. In these references, the high and low fidelity models are deemed qualitatively as such by the modeler. This thesis quantifies the fidelity of a structural model.

Component mode synthesis (CMS) is a method that provides computational advantages by modeling structural components as separate finite element models then assembling their modes to generate a system model [42]. This is a type of reduced-order modeling suitable for complex systems. This method has been applied to many different types of systems, such as damped and undamped systems. An overview of CMS methods was published by Craig in 2002 [22]. The Nyquist fidelity method presented in this paper is different from CMS since the Nyquist fidelity method suggests finite element mesh size for each system component, but creates only one integrated finite element model. 


\subsubsection{Research gap analysis}

The three main related areas of previous work, described in the last sections, are shown graphically in Fig. 14. They all provide methods by which a system progresses from the Conceptual to the Preliminary Design phases. They are summarized as follows:

1. In the first area of research there exist qualitative categorizations of models with differing levels of fidelity. This branch is incomplete and does not suggest ways of producing quantitative models. Also within this area of research is the SVMV method [2], whereby a heuristic rule of thumb is used to make an initial best guess. Then higher and lower meshed models are created. One of these is selected as the final design, depending on their performance. The lines are dashed in this case because a quantitative measure of fidelity is not used even though the concept of fidelity is used in the heuristic.

2. The model reduction techniques generally begin with a high fidelity model, which is complex and computationally expensive, then the model is reduced in order to reduce computation time. The reduced model (and sometimes the high-fidelity model) is used to determine the preliminary design.

3. The adaptive mesh techniques begin with a best guess model, then iterate on it, often refining the mesh until a satisfactory model is created.

The qualitative model fidelity methods (1) are incomplete and have only gone so far as categorizing the models. The research presented in this thesis bridges that gap and use a quantitative metric to create a model with known fidelity in order to predict output metrics of interest. This is shown as the gray area in Fig. 1-4.

\subsection{Context}

The research presented in this thesis provides an alternative method for structural model construction and assessment to the current state-of-the-art, presented in the previous sections. This work focuses on linear structural models of high performance telescopes in the conceptual and preliminary design phases. Three main areas are improved upon or expanded. First, fidelity is quantified using sampling theory in generalized structural component models. This is an advancement in fidelity assessment, which has been largely qualitative. 


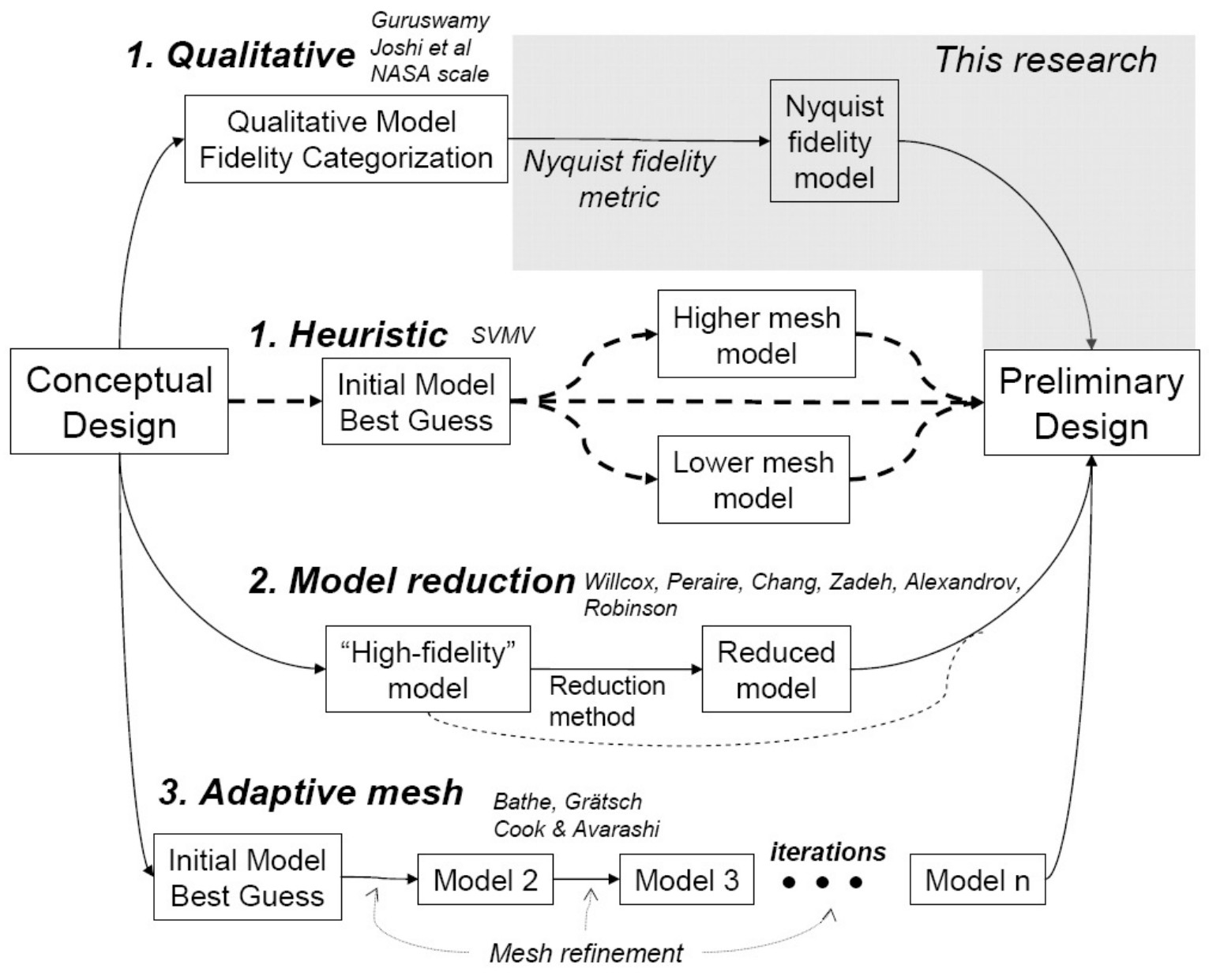

Figure 1-4. Research gap 
Second, the relationship between fidelity and model accuracy is defined for two common structural components in telescope conceptual design models, beams and plates. This relationship enables a rapid accuracy estimate of a given finite element mesh in a known disturbance environment. While there exists finite element error estimators, it has been shown for the examples in Grätsch [36] that error estimators can be expensive to compute (as expensive as analyzing the truth model), or do not always produce provable bounds. It is important to note that the relationship presented in this thesis is empirical based on numerical experiments, but is shown to be computationally efficient in estimating modal accuracy.

Third, the Nyquist fidelity method is developed whereby the fidelity metric is used to produce a finite element mesh a priori with a requested fidelity in order to exhibit the relevant behavior. The mesh fineness is calculated using previously derived numerical relations and therefore is inexpensive to develop, eliminating the need for finely meshed models or a series of different fidelity models against which to gauge the model's appropriateness. Furthermore, since the fidelity is based on sampling theory, the model mesh calculated is able to represent modes within a specified frequency range, not only individual modes.

\subsection{Scope}

The structural models examined in this work are created for inclusion within an integrated modeling framework. Within this framework, one multidisciplinary linear time invariant state space model is created which contains structural, optical, and controls subsystem models. The loading forces are generally modeled as random disturbance forces and torques with known frequency spectra and the disturbance analysis is performed using frequency-domain techniques. This process is further described in Chapter 2. Therefore, this work focuses on systems modeled as linear and for systems with quasi-steady state loading represented as an arbitrary, but known, frequency spectra.

Moreover, the fidelity method is designed to be most useful during the conceptual design phase where many configurations of a system are considered and where optimization, sensitivity, and uncertainty analyses are performed. In supporting the effort to move more analysis into the conceptual design phase, a Nyquist method is created whereby models of many configurations can be rapidly developed using the guidelines presented here. In lieu of hardware models, generally not present during the conceptual design phase, high-fidelity models are usually taken as the truth models. In order to reduce the development and 
computation time required for several high-fidelity models, the Nyquist fidelity method provides a rapid means of constructing sufficient fidelity models where a specified degree of model accuracy is assured.

Lastly, this thesis provides a guideline for mesh densities of common structural finite element components used for dynamic loading analysis. It does not provide a provable finite element error estimate, but rather a modeling method which ensures relevant flexural behavior is captured in the finite element model.

\subsection{Thesis Overview}

The layout of the thesis is as follows. Chapter 2 describes the integrated modeling environment, the nature of the disciplinary models including the structural model, and the disturbance analysis methods. The spacecraft telescope example, MOST, is also introduced here.

Chapter 3 describes fidelity and quantifies fidelity using the Nyquist criterion for plates and beams. Also presented is the fidelity assessment method, based on the fidelity metric.

The Nyquist fidelity method of constructing models from geometric and material considerations is detailed in Chapter 4. An example of this method on the MOST system is also shown.

Chapter 5 demonstrates how the fidelity method can be applied to two real world telescope systems, TMT and SOFIA.

Finally Chapter 6 lists recommendations for best practices for large-scale, multi-user, model management based on field observations, enumerates the unique and important contributions of this thesis, and suggests future work. 


\section{Chapter 2}

\section{Integrated Modeling and Analysis}

\section{Framework}

This chapter describes the modeling and analysis methods used in the integrated modeling framework. This framework is an end-to-end simulation designed to capture the interaction behavior between subsystems, model the effect of disturbance loading, and estimate and assess performance metrics. This is the modeling context in which the fidelity metric and methods are presented and demonstrated, therefore a brief overview of the integrated modeling framework is presented. First, the concept of an integrated model is introduced and the analysis process of a parameterized integrated model is shown. Next, different types of performance analyses are described. Finally, the MOST system is introduced and its integrated model is described in detail.

\subsection{Integrated Modeling}

The purpose of an integrated model is to capture in one model the interaction between relevant subsystems, to estimate performance metrics and to identify trends in multidisciplinary system designs. This model helps make design decisions (such as in optimization, uncertainty or sensitivity analyses) or to predict system behavior during operations. Gutierrez [39] provides an excellent description of the discipline models and application of the integrated modeling process. An example is given by de Weck and Miller [24], [60]. [61], [27]. 


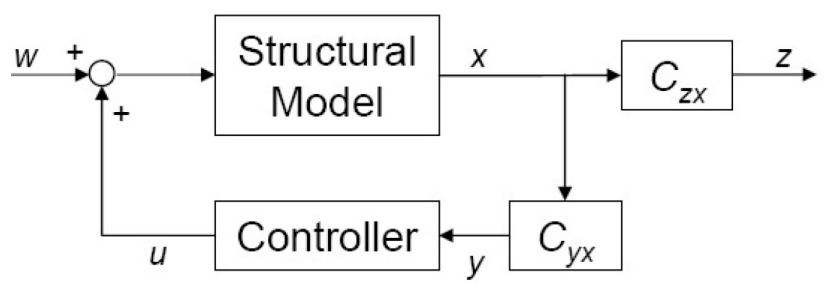

Figure 2-1. Example of an integrated model

who apply the integrated modeling and analysis procedure to the Next Generation Space Telescope and the Space Interferometry Mission. In this thesis, high performance telescope systems are analyzed, and the discipline models of structures, optics, and controls are generally included in such an integrated model. A thermal model can be included as well, depending on the surrounding environment.

Figure 2-1 is an example of an integrated model containing a structural model and a control model, and disturbance inputs, $w$. The model outputs, $z$, are calculated as a linear combination of the structural states, $x$, using the matrix $C_{z x}$. In a telescope system, the matrix $C_{z x}$ is often a linear sensitivity matrix representing the optical system. The matrix $C_{y x}$ converts system states into sensor measurements, $y$. The controller produces the the control inputs, $u$ from the sensor measurements, $y$. In state space form, the closed loop state space model in Fig. 2-1 is represented as

$$
\begin{aligned}
& \dot{q}=A_{z w} q+B_{z w} w \\
& z=C_{z w} q
\end{aligned}
$$

where $q$ are the system states and $A_{z w}, B_{z w}$, and $C_{z w}$ are the system state space matrices.

Furthermore, this integrated model can be parameterized such that configuration, geometry and material properties can be varied conveniently by changing parameter values in an input file. Using a parameterized model enables the rapid evaluation of many different designs. Uebelhart [78] describes the parameterization process in detail and provides the flow chart in Fig. 2-2. In the process, the parameters are first set in the main input file. Next, the finite element structural model input data is assembled in Matlab ${ }^{\circledR}$ according to the parameter values. Then, a finite element normal modes analysis is performed on the model and the results are used to assemble the structural plant within the integrated model. Finally, the DOCS ${ }^{\circledR}$ (Disturbance-OpticsControls-Structures) toolbox assists in a variety of performance analyses, such as optical performance and 


\section{Design variable inputs}

- Structural form, dimensions

- Material / geometry properties

- Sub-system parameters

\section{Output metrics}

- Optical performance

- Mass

- Uncertainty bounds

\begin{tabular}{|l|l|}
\multicolumn{1}{|c|}{ MATLAB } & DOCS \\
\hline $\begin{array}{l}\text { Integrated model } \\
\text { build \& assembly }\end{array}$ & $\longrightarrow \begin{array}{l}\text { Analysis } \\
\text { routines }\end{array}$ \\
\hline
\end{tabular}

Figure 2-2. Analysis process of parameterized models. Source: [78]

mass estimation. The DOCS toolbox is maintained by Nightsky Systems 1 and enables automatic generation and analysis of integrated models from within the Matlab programming environment as shown in Fig. 2-2 in addition to its analysis capabilities. Several telescope systems have been modeled and analyzed using the techniques available in DOCS. The models shown in this work are produced and analyzed using the DOCS toolbox. The fidelity techniques in this work are intended to add to the DOCS capability.

The parameterized model is particularly useful in iterative analyses, such as optimization, due to its automatic generation and analysis capability.

\subsection{Performance Analysis}

In this section, a set of performance analyses commonly used on integrated models is described. First, two types of frequency domain analyses are described. 2 Then, a common model reduction technique and a sensitivity analysis technique are presented. These are described in detail here since these analyses are used later in this thesis.

The set of analyses shown here is not an exhaustive list, but it does describe the techniques used in this thesis. The thesis by Gutierrez [39] provides the foundation of integrated modeling and of performance analysis techniques. Recent additions to integrated modeling techniques such as isoperformance, tailoring/tuning, and uncertainty analysis can be found in de Weck [25], [26], Masterson [56], [57], and Uebelhart [78], [80], respectively.

\footnotetext{
${ }^{1}$ www.nightsky-systems.com

${ }^{2}$ Integration techniques for time-domain analyzes are not presented here, the reader is referred to de Weck [25] and Gutierrez [39] for a discussion of time-domain analysis
} 


\subsubsection{Frequency domain analysis}

Frequency domain analysis methods are used since they provide a computationally efficient way to evaluate the behavior of a linear time-invariant (LTI) system. The telescope models addressed in this thesis are assumed to be LTI systems because they are in quasi-steady-state conditions during observations, meaning that there are small forces and torques indicating small displacements. For transient disturbances or highly non-linear systems, time-domain analysis methods are more appropriate. There are two types of frequency domain analysis techniques for estimating output figures of merit (OFM) used for integrated models. The first technique is the PSD integration method and the second is Lyapanov analysis [17].

In the PSD integration method, the root-mean-square (RMS) of the OFM is calculated. This quantity is labeled $\sigma_{z_{i}}$ and is the $i^{t h} \mathrm{OFM}$ in the case of multiple output metrics. First, the system transfer function is calculated from the state space integrated model matrices from Eqn. 2.1 and is expressed as a function of frequency, $\omega$,

$$
G_{z w}(j \omega)=C_{z w}\left(j \omega I-A_{z w}\right)^{-1} B_{z w}+D_{z w}
$$

These state space matrices include the closed loop dynamics of the controllers and relate the input disturbances, $w$ to the output figures of merit, $z$. Next, the power spectral density (PSD) of the output metrics, $S_{z z}$, is calculated from the transfer function, $G_{z w}$, and the PSD of the disturbance forces, $S_{w w}$.

$$
S_{z z}(j \omega)=G_{z w}(j \omega) S_{w w}(j \omega) G_{z w}^{H}(j \omega)
$$

The PSD of the disturbance forces is generally given and is discussed later in Section 2.3.4. The quantities $S_{z z}, G_{z w}$, and $S_{w w}$ are each a function of frequency and are multidimensional in the case of multiple input/multiple output system. Finally, $S_{z z}$ is integrated over all frequencies in order to calculate the state covariance matrix,

$$
\Sigma_{z z}=\frac{1}{2 \pi} \int_{-\infty}^{\infty} S_{z z}(j \omega) d \omega
$$

In practice, we only integrate over finite frequency bounds, corresponding to the frequencies over which the 
model and disturbances are defined. The mean-square values of the OFM are on the diagonal,

$$
\Sigma_{z z}=\left[\begin{array}{ccc}
\ddots & & 0 \\
& \sigma_{z_{i}}^{2} & \\
0 & & \ddots
\end{array}\right]
$$

The RMS is simply the root of these quantities. It should be noted that the units of $\omega \mathrm{are} \mathrm{rad} / \mathrm{s}$. The quantity $S_{z z}$ is also called the output PSD and it provides dynamic response of the state space model output across all frequencies defined. Since the RMS depends on the integral of this quantity over frequency, the frequency content of $S_{z z}$ provides valuable insight into what frequencies contribute most to the OFM, $\sigma_{z_{i}}$.

The Lyapanov analysis also calculates the RMS of the OFM. However, instead of modeling the disturbance as a PSD, the disturbance is approximated as a shaping filter on white noise, producing "colored" noise. The disturbance can then be modeled as a state space system and augmented to the closed loop plant model equations from Eqn. 2.1. The resulting augmented system can be seen in Fig. 2-3 and written as:

$$
\begin{aligned}
& \dot{q}=A_{z d} q+B_{z d} d \\
& z=C_{z d} q
\end{aligned}
$$

where the inputs, $d$ represent white noise and the outputs, $z$ are the familiar OFM. The state covariance matrix, $\Sigma_{z}$, is then calculated using the steady-state Lyapanov equation,

$$
A_{z d} \Sigma_{z z}+\Sigma_{z z} A_{z d}^{T}+B_{z d} B_{z d}^{T}=0
$$

Finally the mean-square of the OFM is found by taking the trace of the covariance matrix,

$$
\sigma_{z_{i}}^{2}=\operatorname{trace}\left[\Sigma_{z z}\right]
$$

The Lyapanov analysis provides an efficient way of estimating RMS performance. However, unlike the PSD integration method, no insight into the frequency content of the performance metric is gained. In addition, the PSD integration method is frequently used because the disturbance cannot always be modeled efficiently, i.e. using only a few states, using a white noise filter. 


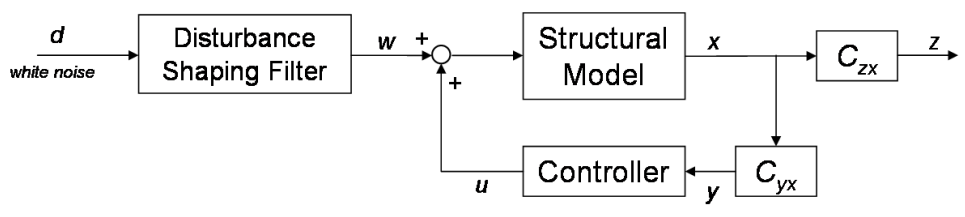

Figure 2-3. Augmented system diagram

\subsubsection{Model reduction}

Model reduction is performed on complex models such as telescopes since performance analysis of full models is computationally expensive. Many model reduction techniques are available. The reader is referred to Antoulas [8] for a survey of techniques. The model reduction used in this work is the Balanced Reduction method based on Hankel Singular Values (HSV), which is a very common method [66] [64].

Balanced Reduction is achieved by balancing the controllability and observability grammians of the transformed integrated model states. This is done by finding a similarity transform matrix, $T$, which, when applied to the integrated model, will produce equal controllability and observability grammians. Moore [64] and Laub [46] provide methods by which $T$ is found. It is important to note that the similarity transform does not alter the system's transfer functions. Furthermore, balanced reduction is used when the disturbance can be represented as a white noise shaping filter as in Fig. 2-3 and Eqn. 2.6. The transformed system can be written as

$$
\begin{aligned}
& \dot{\tilde{q}}=T A_{z d} T^{-1} \tilde{q}+T B_{z d} d \\
& z=C_{z d} T^{-1} \tilde{q}
\end{aligned}
$$

where the transformed state vector, $\tilde{q}$ is

$$
\tilde{q}=T q
$$

The balanced observability and controlability grammians, $\tilde{W}_{c}$ and $\tilde{W}_{o}$, are given as,

$$
\tilde{W}_{c}=T W_{c} T^{T}=\tilde{W}_{o}=\left(T^{-1}\right)^{T} W_{o} T^{-1}
$$

where $W_{c}$ and $W_{o}$ are the controlability and observability grammians of the untransformed system and are 


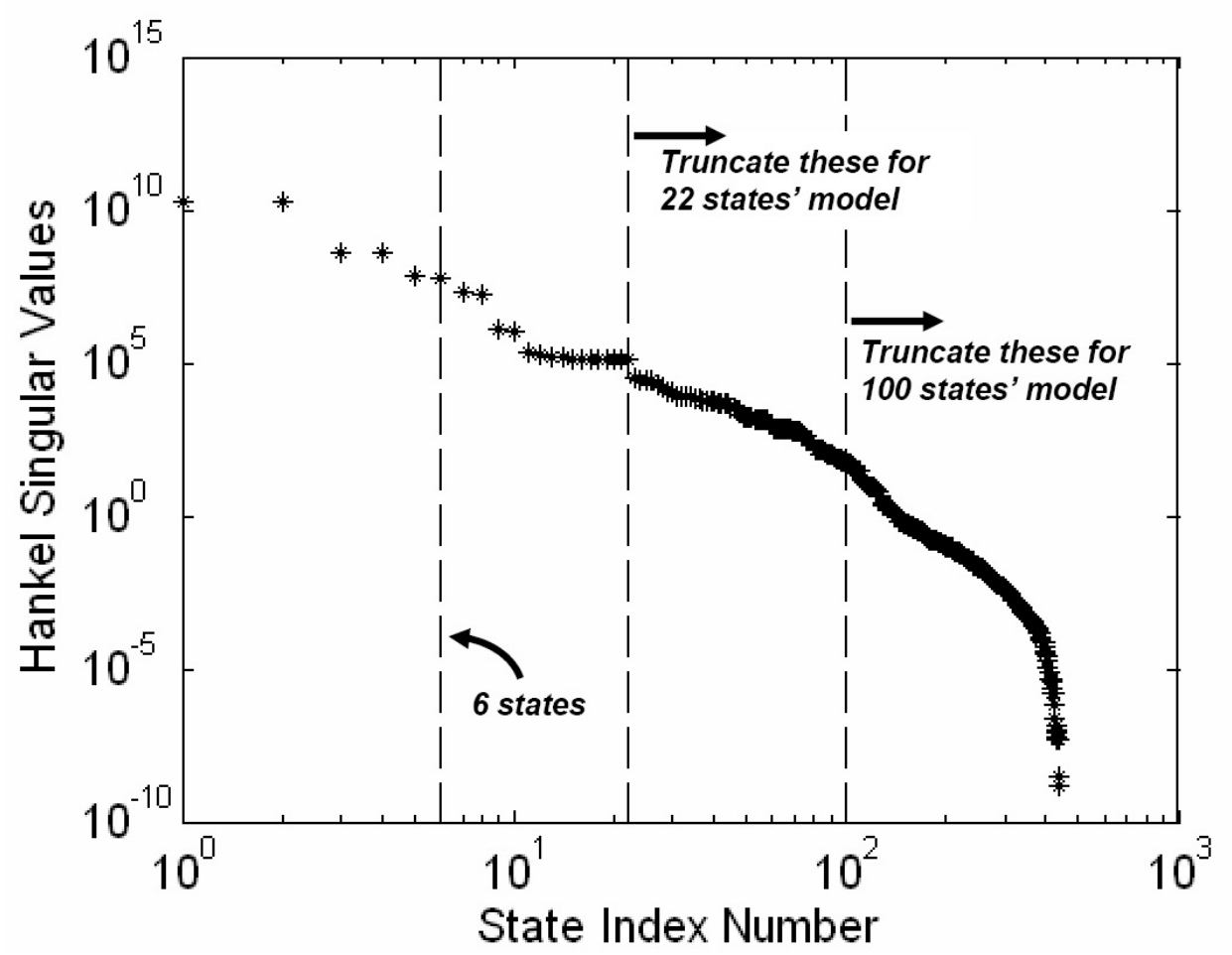

Figure 2-4. Hankel singular values of MOST model

calculated as

$$
\begin{aligned}
& A_{z d} W_{c}+W_{c} A_{z d}^{T}+B_{z d} B_{z d}^{T}=0 \\
& A_{z d}^{T} W_{o}+W_{o} A_{z d}+C_{z d} C_{z d}^{T}=0
\end{aligned}
$$

The transformed grammians rank the states in decreasing order of observability and controllability.

Next, the HSVs are calculated for each state by taking the diagonals of $\tilde{W}_{c}$ and $\tilde{W}_{o}$. Then, the states corresponding to low Hankel singular values, i.e. states with low observability and controlability, are truncated.

Figure 2-4 shows the HSVs for the MOST model. The first 6 states correspond to the rigid body modes. Two example truncations are shown at 22 modes and 100 modes. The truncated states are reflected in truncated state space matrices. More information on balanced state reduction can be found in Gawronski [33].

The decision of where to truncate the states can also be made by specifying the RMS error incurred by 
truncating the states. de Weck [25] derives the RMS error bound for truncation,

$$
\frac{\Delta \sigma_{z_{i}}}{\bar{\sigma}_{z_{i}}} \leq \frac{\sum_{i=n_{k}+1}^{n_{s}} \sigma_{i}^{H}}{\sum_{i=1}^{n_{k}} \sigma_{i}^{H}}
$$

where the left hand side is the relative RMS performance error, $n_{k}$ is the number of states retained, $n_{s}$ is the total number of states, and $\sigma_{i}^{H}$ is the $i^{\text {th }} \mathrm{HSV}$. The value $n_{k}$ is chosen so that the RMS error is bounded.

\subsubsection{Sensitivity analysis}

This section describes methods used to find the sensitivity of output figures of merit with respect to modal parameters. These techniques are used to relate the modal frequency error predicted by fidelity to the system's output figure of merit. Two sensitivity analyses are used, one for each of the frequency domain performance analyses.

Uebelhart [78] derives the sensitivity of the RMS of the OFM for the PSD integration method with respect to the modal parameters. Using the chain rule the RMS OFM sensitivities can be written as

$$
\frac{\partial \sigma_{z_{i}}}{\partial p}=\frac{1}{2 \sigma_{z_{i}}} \frac{\partial \sigma_{z_{i}}^{2}}{\partial p}
$$

where $\sigma_{z_{i}}$ is the RMS OFM and $p$ is a modal parameter. The mean square sensitivities are on the diagonals of the derivative of the $\Sigma_{z z}$ matrix,

$$
\frac{\partial \Sigma_{z z}}{\partial p}=\left[\begin{array}{ccc}
\ddots & & 0 \\
& \frac{\partial \sigma_{z_{i}}^{2}}{\partial p} & \\
0 & & \ddots
\end{array}\right]
$$

This is the $\Sigma_{z z}$ matrix from Eqn. 2.4 Its derivative is given by

$$
\frac{\partial \Sigma_{z z}}{\partial p}=\frac{1}{\pi} \int_{0}^{+\infty}\left(\frac{\partial G_{z w}}{\partial p} S_{w w} G_{z w}^{H}+G_{z w} S_{w w} \frac{\partial G_{z w}^{H}}{\partial p}\right) d \omega
$$

The sensitivity of the transfer function matrix with respect to the modal parameters, $\frac{\partial G_{z w}}{\partial p}$, is calculated 
using a linear fractional transformation and is derived in Uebelhart [78].

The modal parameter sensitivities when using the Lyapanov analysis are derived in Gutierrez [39]. The sensitivity of the $i^{\text {th }}$ RMS OFM with respect to a modal parameter, $p$, is given by

$$
\frac{\partial \sigma_{z_{i}}}{\partial p}=\frac{1}{2 \sigma_{z_{i}}} \operatorname{trace}\left[L_{i}\left(\frac{\partial A_{z d}}{\partial p} \Sigma_{q}+\Sigma_{q} \frac{\partial A_{z d}^{T}}{\partial p}\right)\right]
$$

where $A_{z d}$ is the matrix in Eqn. 2.6, $\Sigma_{q}$ is the solution to the Lyapanov equation 2.7 and $L_{i}$ is a symmetric Lagrange multiplier matrix. The Lagrange matrix is found by solving another Lyapanov equation,

$$
L_{i} A_{z d}+A_{z d}^{T} L_{i}+C_{i}^{T} C_{i}=0
$$

where $C_{i}$ is the $i^{t h}$ row of the $C_{z d}$ matrix. When $p$ is chosen as the $i^{t h}$ modal frequency,

$$
p=\omega_{i}
$$

the derivative of the matrix is determined by inspection of Eqn. 4.41 in Gutierrez [39] and is

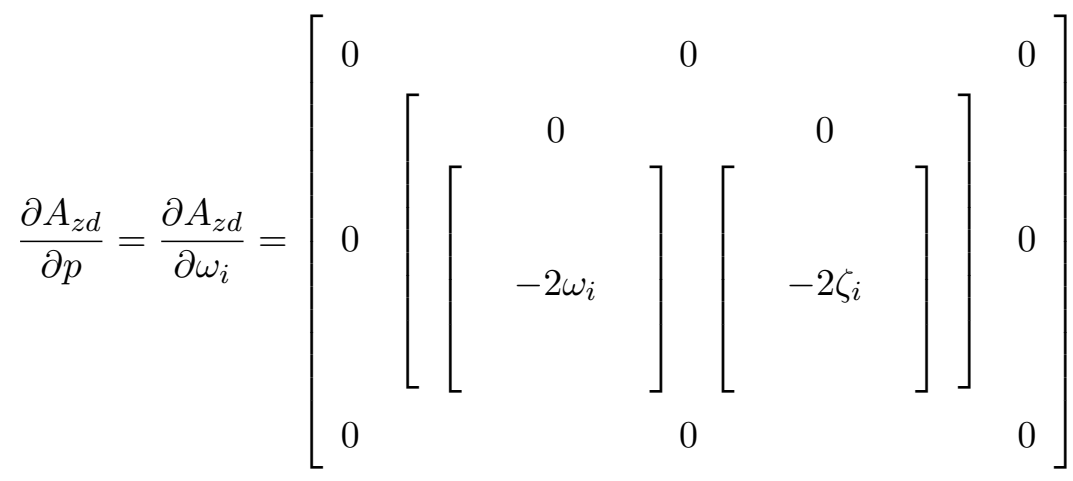

In Chapter 3, the modal frequency error of a system due to the spatial discretization of the FE mesh is estimated. The sensitivity analysis presented in this section relates the change in modal frequency error to the change in the output figure of merit. In this way, the error in an OFM estimate due to the size of the FE is determined. The procedure is described in Section 3.5 


\subsection{Modular Optical Space Telescope}

The Modular Optical Space Telescope (MOST) is the main case study in this thesis and will be introduced in this section. MOST is a spacecraft telescope designed to image targets in the optical and infrared wavelengths. The nominal configuration of MOST is a Cassegrain telescope with a 3 meter diameter primary mirror and a tripod support for the secondary mirror support tower, as shown in Fig. 2-5. The solar panels provide power and the bus provides stationkeeping and communication functions and houses the focal plane instruments. There are two isolators not shown in this figure, one between the reaction wheel assembly (RWA) and the bus and one between the bus and the OTA.

MOST is currently in the conceptual design phase and a parametric model has been developed to investigate tradeoffs between several design parameters and performance. This is part of an effort to move design analysis from the preliminary design phase to the conceptual design phase. The major design parameters are listed in Table 2.1. The ranges of the parameter values shown are based on mission specific requirements or are best guess estimates, such as modal damping. Note that the first six parameters change the configuration of the telescope. For example, changing the optical prescription changes the distance between the primary and secondary mirrors and therefore changes the size of the secondary tower. Some of these alternate designs are shown in Uebelhart [78] and are reproduced in Fig 2-6. In addition to these parameters, any material property or dimension is also a parameter. For example parameters in the model are the stiffness and volumetric mass density of the secondary support tower beams. A more in depth description of the MOST parametric model and its use in performing trade studies can be found in Uebelhart [78].

Table 2.1. MOST design parameters

\begin{tabular}{lc}
\hline \hline Parameters & Values \\
\hline Mirror Configuration & Monolithic,Segmented \\
Mirror Type & Flat or Rib-stiffened \\
SST Type & Tripod with or without crossbars \\
Primary mirror diameter & $1-5$ meters \\
$f$-number & $1.0-2.0$ \\
Final focal ratio & $15-22$ \\
Modal damping & $0.5-1.0 \%$ \\
RWA isolator corner frequency & $10 \mathrm{~Hz}$ \\
Bus isolator corner frequency & $2 \mathrm{~Hz}$ \\
\hline \hline
\end{tabular}




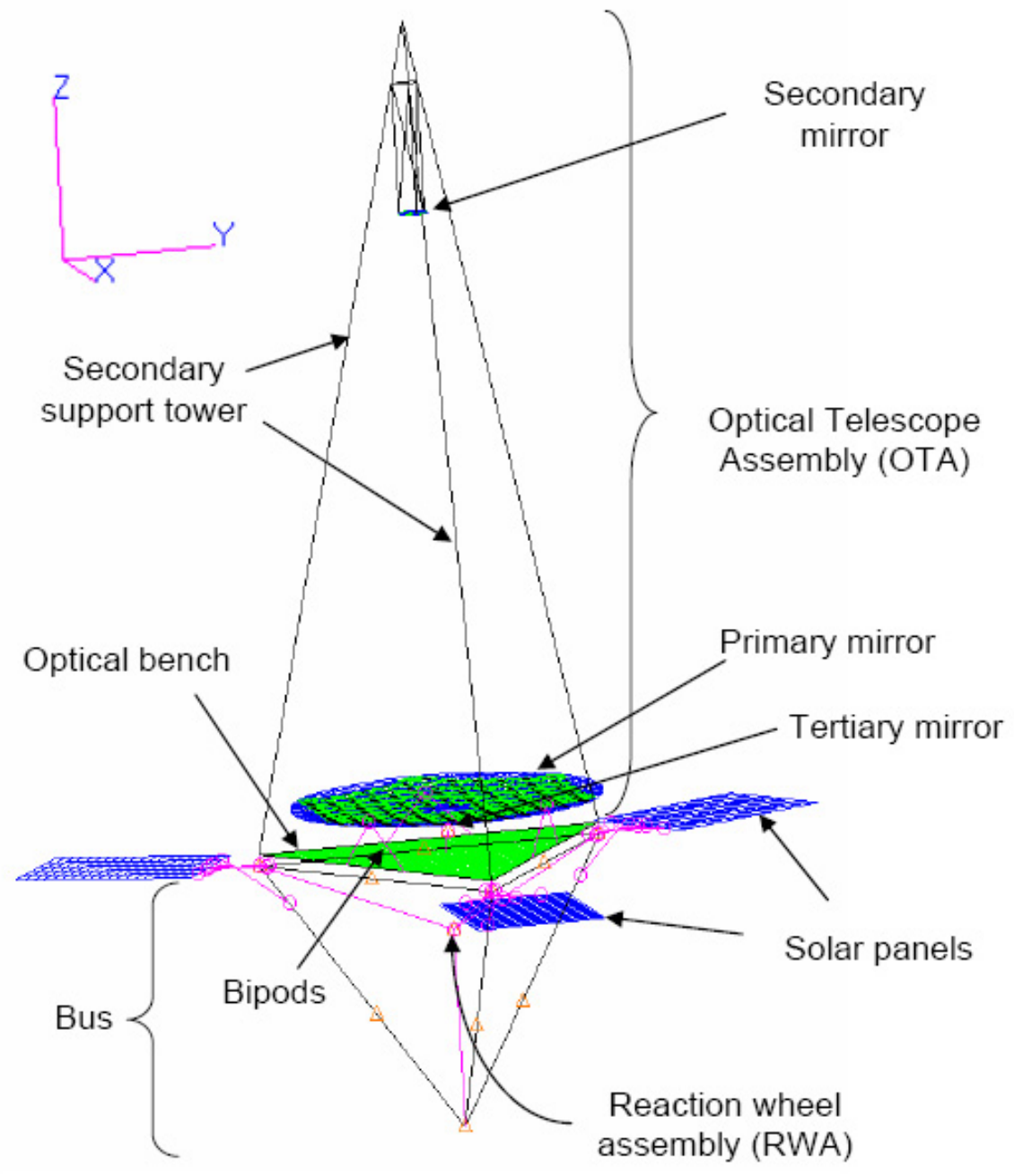

Figure 2-5. MOST nominal configuration 


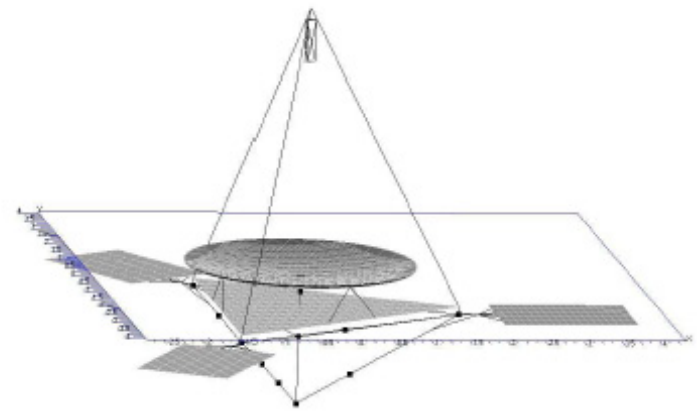

(a) 3 meter $f / 1.0$ monolithic mirror

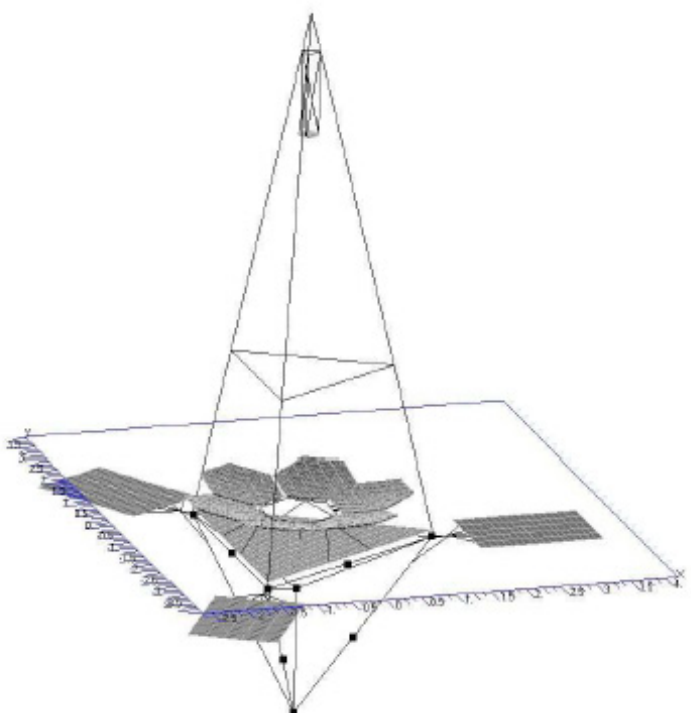

(b) 3 meter $f / 2.0$ segmented mirror with crossbars on the SST

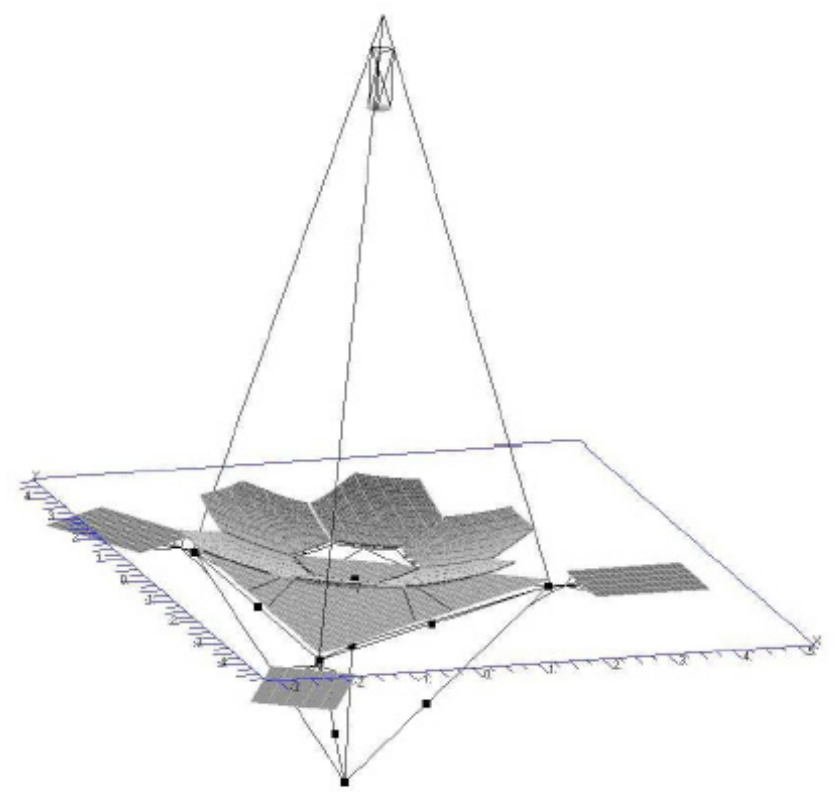

(c) 5 meter $f / 1.5$ segmented mirror

Figure 2-6. Alternate MOST configurations. Source: [78] 


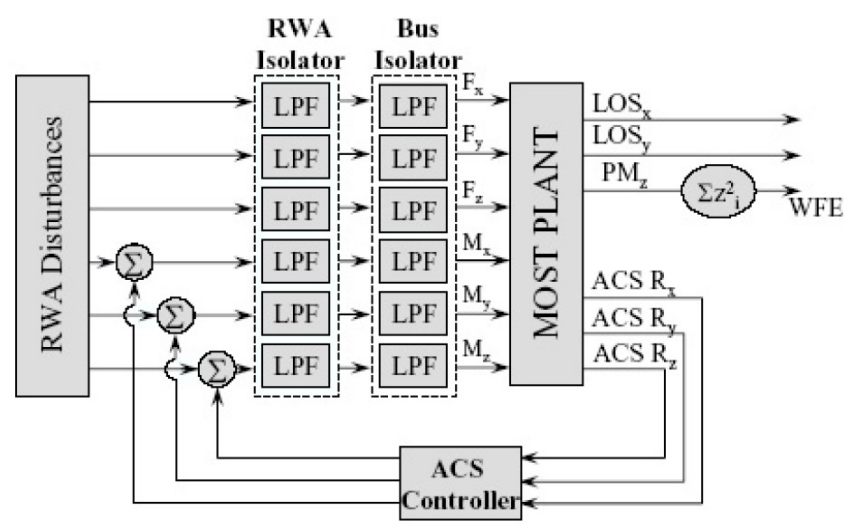

Figure 2-7. MOST integrated model block diagram [78]

The MOST model is a multidisciplinary integrated model and also contains optical, controls, and disturbance models in addition to the structural model. The integrated model is discussed in the next section.

\subsubsection{Integrated model}

A schematic of the MOST integrated model can be seen in Fig. 2-7, reproduced from Uebelhart [78]. This is similar to the general diagram in Fig. 2-1. The MOST plant consists of the combined optical and structural models, further discussed in the next sections. The outputs of the plant are an optical metric (Line of sight jitter in the x- and y-directions, $L O S_{x}, L O S_{y}$ ), the primary mirror displacements, $P M_{z}$, and the rotations of the spacecraft in three directions used for the attitude control system $\left(A C S_{R x}, A C S_{R y}, A C S_{R z}\right)$. Another optical metric, the wavefront error (WFE), can be calculated by summing the squares of the Zernike coefficients, $z_{i}$, which are calculated from the primary mirror displacements. The attitude controller commands the reaction wheels to impart torque to the spacecraft for slewing maneuvers. The source of the reaction wheel disturbance is imbalance in the reaction wheels which causes forces and torques on the system through the RWA [39]. These disturbances are attenuated via the RWA and bus isolators. In this model both isolators are modeled using low pass filters (LPF). Also present, but not shown in this diagram are the deformable mirror controller and the fast steering mirror (FSM) controller. For more details on the control design of MOST, the reader is referred to Cohan [19]. The structural, optical and disturbance models are described in the next sections. 


\subsubsection{Structural model}

As discussed above, the structural MOST model is formed using the first three blocks of the flow diagram in Fig. 2-2 by setting the design parameters, running the automatic finite element input file and analyzing the finite element model using the normal modes analysis, which represents the homogenous solution. During the "DOCS Analysis routines" block, the natural frequencies and modeshapes ( $\Omega$ and $\Phi$, respectively) are brought back into Matlab using DOCS. The structural model is then assembled using the techniques below and analyses are performed.

The structural state space system model can be made in many different ways. The second order modal form shown in Gawronski [32] is used to build the state space model which relates force and torque input to displacement and velocity outputs,

$$
\begin{aligned}
& \dot{\mathrm{x}}=\mathbf{A x}+\mathbf{B}_{\mathbf{u}} \mathrm{u}+\mathbf{B}_{\mathbf{w}} \mathrm{w} \\
& \mathrm{y}=\mathbf{C x}+\mathbf{D}_{\mathbf{u}} \mathrm{u}+\mathbf{D}_{\mathbf{w}} \mathrm{w}
\end{aligned}
$$

where $x$ is the $2 m \times 1$ modal state vector containing the displacements and velocities of each mode, and $m$ is the number of natural modes extracted from the FEA. The vectors $u$ and $w$ represent the input command forces and disturbance forces, respectively.

The displacements and velocities of each node, $q$ and $\dot{q}$ respectively, can be recovered from the states by using the $n \times m$ modeshape matrix $\Phi$.

$$
\left\{\begin{array}{c}
q \\
\dot{q}
\end{array}\right\}=\Phi \mathrm{x}
$$

The input forces enter into the system via the input forcing vector $u$ which is of size $n \times 1$ where $n$ is the number of degrees of freedom in the structure. Disturbance forces enter the system through the $n \times 1$ vector, $w$. The vector $y$ is the output vector and is of size $o \times 1$ where $o$ is the number of outputs. The system matrices, $\mathbf{A}, \mathbf{B}$, and $\mathbf{C}$ relate the input forces to the outputs. They are

$$
\begin{aligned}
\underset{2 m \times 2 m}{\mathbf{A}} & =\left[\begin{array}{cc}
0 & I \\
-\omega^{2} & -2 \zeta \omega
\end{array}\right] \underset{2 m \times n}{\mathbf{B}_{\mathbf{u}}}=\left[\begin{array}{c}
0 \\
\Phi^{\mathrm{T}} B_{n u}
\end{array}\right] \underset{2 m \times n}{\mathbf{B}_{\mathbf{w}}}=\left[\begin{array}{c}
0 \\
\Phi^{\mathrm{T}} B_{n w}
\end{array}\right] \\
\underset{o \times 2 m}{\mathbf{C}} & =\left[\begin{array}{ll}
C_{n q} \Phi & C_{n \dot{q}} \Phi
\end{array}\right]
\end{aligned}
$$


The A matrix contains the square matrices $-\omega^{2}$ and $-2 \zeta \omega$ which contain the individual modal frequencies and damping ratios, $\omega_{i}$ and $\zeta_{i}$ on the diagonals. Adding modal damping in this manner is a common way to approximate the damping in the system. The matrices $B_{n u}$ and $B_{n w}$ map the input degrees of freedom to the input vectors $u$ and $w$ respectively, and are transformed from nodal coordinates to modal coordinates using the $\Phi$ matrix. The $\mathbf{C}$ matrix relates the modal states to the outputs and is comprised of the matrices $C_{n q}$ and $C_{n \dot{q}}$ which contain pointing vectors to the appropriate modal displacement and velocity degrees of freedom, respectively. They are transformed into nodal coordinates using the $\Phi$ matrix. The feed-forward matrices $\mathbf{D}_{\mathbf{u}}$ and $\mathbf{D}_{\mathbf{w}}$ are usually zero and are ignored for the analyses in this thesis. Therefore, the outputs, $y$, in Eqn. 2.21 contain the nodal displacements and velocities.

\subsubsection{Optical model}

The optical model describes how the movements of the optical structures affect the optical performance metrics. Some common optical performance metrics are optical pathlength difference (OPD), wave front error (WFE) and line-of-sight jitter (LOS) . The optical pathlength difference is used in interferometry and in optical systems. In interferometric systems it measures the difference in length the science light travels through two different apertures. This is a key performance metric in interferometry because in order to achieve interferometric nulling, the OPD must be on the order of a fraction of a wavelength. For the optical/infrared wavelengths, this translates to a few nanometers. In optical systems, OPD measures the deviation of the image from the expected perfect spherical wave at the exit pupil. For the MOST system, LOS and WFE are calculated.

The LOS metric measures how off-center the image is due to disturbance effects and is calculated directly from the translational and rotational displacements of the primary, secondary, and tertiary (FSM) mirrors. The calculations for LOS in the $\mathrm{x}$ - and $\mathrm{y}$-directions in the image plane can be found in a memo by Perrygo and Burg [70] and are,

$$
\begin{aligned}
& L O S_{x}=-\frac{1}{f_{1}} \delta_{P_{y}}+\frac{(M-1)}{M f_{1}} \delta_{S_{y}}+\frac{1}{M f_{1}} \delta_{T_{y}}+2 \alpha_{P_{x}}-\frac{2}{M+1} \alpha_{S_{x}}-\frac{2}{M+1} \alpha_{T_{x}} \\
& L O S_{y}=\frac{1}{f_{1}} \delta_{P_{x}}-\frac{(M-1)}{M f_{1}} \delta_{S_{x}}-\frac{1}{M f_{1}} \delta_{T_{x}}+2 \alpha_{P_{y}}-\frac{2}{M+1} \alpha_{S_{y}}-\frac{2}{M+1} \alpha_{T_{y}}
\end{aligned}
$$

where $\delta$ and $\alpha$ are the displacements and rotations, respectively, of the primary (P), secondary (S), and 
tertiary $(\mathrm{T})$ mirror center points in or about the $\mathrm{x}$ - and $\mathrm{y}$-directions in the image plane. The optical parameters included are the focal length of the primary mirror, $f_{1}$, and the secondary mirror magnification, $M$. LOS is an angular measurement.

Since these equations represent operations directly on the structural model outputs, the LOS jitter calculation can be incorporated directly into the structural model in order to make the outputs in $z$ reflect the LOS metrics. First, a matrix, $C_{L O S}$, is created which transforms the relevant translational and rotational nodal displacements to the LOS metrics,

$$
\begin{aligned}
C_{L O S}= & {\left[\begin{array}{ccccccccccccc}
0 & -1 / f_{1} & 0 & 2 & 0 & 0 & 0 & \frac{(M-1)}{M f_{1}} & 0 & -\frac{2}{M+1} & 0 & 0 & \ldots \\
1 / f & 0 & 0 & 0 & 2 & 0 & -\frac{(M-1)}{M f_{1}} & 0 & 0 & 0 & -\frac{2}{M+1} & 0 & \\
0 & \frac{1}{M f_{1}} & 0 & -\frac{2}{M+1} & 0 & 0 \\
& -\frac{1}{M f_{1}} & 0 & 0 & 0 & 0 & -\frac{2}{M+1}
\end{array}\right] }
\end{aligned}
$$

where

$$
\left[\begin{array}{c}
\operatorname{LOS}_{x} \\
\operatorname{LOS}_{y}
\end{array}\right]=C_{L O S}\left[q_{\text {subset }}\right]
$$

and

$$
\left[q_{\text {subset }}\right]=\left[\delta_{P_{x}}, \delta_{P_{y}}, \delta_{P_{z}}, \alpha_{P_{x}}, \alpha_{P_{y}}, \alpha_{P_{z}}, \delta_{S_{x}}, \delta_{S_{y}}, \delta_{S_{z}}, \alpha_{S_{x}}, \alpha_{S_{y}}, \alpha_{S_{z}}, \delta_{T_{x}}, \delta_{T_{y}}, \delta_{T_{z}}, \alpha_{T_{x}}, \alpha_{T_{y}}, \alpha_{T_{z}}\right]^{\mathrm{T}}
$$

This is a linear sensitivity matrix that usually contains acceptable accuracy and is calculated using finite differencing optical modeling code (ray tracing).

Next, the $\mathbf{C}$ matrix, shown in Equations 2.21 and 2.23, is constructed such that $C_{n q}$ points to the nodal degrees of freedom in Eqn. 2.27. Next, $\mathbf{C}$ is premultiplied by the matrix $C_{L O S}$ which gives the LOS outputs, $z$. The augmented structural system can be written as

$$
\begin{aligned}
& \dot{\mathrm{x}}=\mathrm{Ax}+\mathrm{B}_{\mathrm{u}} \mathrm{u}+\mathrm{B}_{\mathrm{w}} \mathrm{w} \\
& \mathrm{y}=\mathrm{Cx} \\
& \mathrm{z}=C_{L O S} \mathrm{Cx}
\end{aligned}
$$


WFE measures the deviation of the wavefront from planar after it has traveled through the telescope (i.e. at the detector). This is possible due to the assumption that the wavefront of the science light entering the telescope is planar. WFE is created from distortions in the optical surfaces due to thermal or mechanical disturbance effects.

The WFE is measured using Zernike polynomials [16], which are a set of two dimensional basis functions orthogonal over the unit circle which describe deformations in the wavefront. The first few Zernike functions and their shapes are shown in Fig. 2-8. where $\rho$ and $\varphi$ are the radial and circumferential compo-
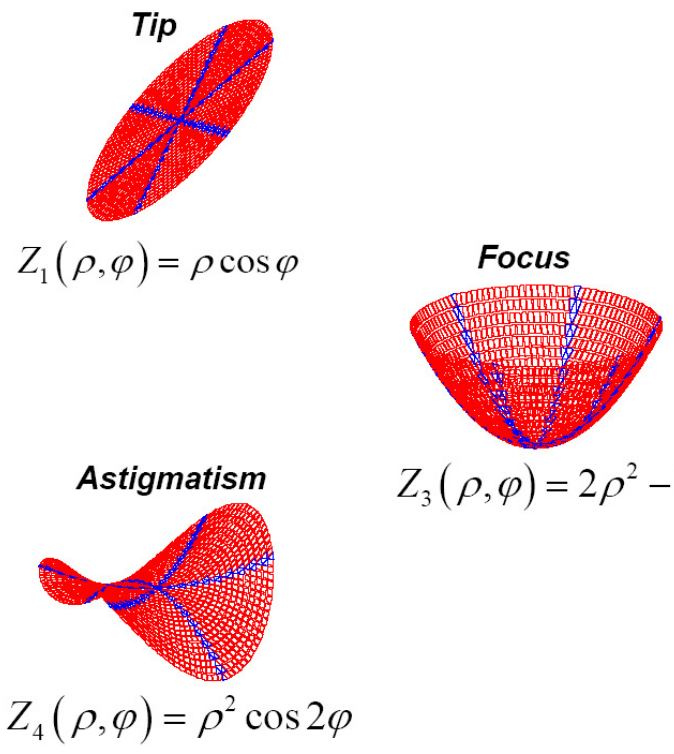

$$
Z_{3}(\rho, \varphi)=2 \rho^{2}-1
$$

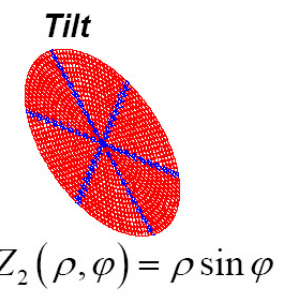

Figure 2-8. First 5 Zernike functions

nents, respectively. The numbering of the Zernikes can be arbitrary. However, according to convention, the numbering follows either the "fringe," used at the University of Arizona [6] or the Noll numbering system. The list above uses the fringe numbering. While there are an infinite number of Zernike functions, the first 36 Zernikes are of low $\rho$ and $\varphi$ orders and are usually considered sufficient for most deformations encountered in mirror dynamics. The deviation from a flat circular plate can be described as a linear combination of Zernike functions. The complete set of Zernike functions is found in Born and Wolf [16].

The coefficients of the linear combination of Zernike functions describing a deformed circular surface 
are labeled $z_{i}$ in Fig. 2-7 and determine the root mean square (RMS) of the WFE,

$$
\mathrm{WFE}_{\mathrm{RMS}} \simeq \sqrt{\sum_{i=1}^{N_{Z}} \frac{z_{i}^{2}}{\alpha_{i}}}
$$

where $N_{Z}$ are the number of included Zernike coefficients, $z_{i}$ is the $i^{\text {th }}$ Zernike coefficient and $\alpha_{i}$ is the corresponding weight. The weights are assigned to each Zernike functional in order to normalize the RMS. More information on Zernike formulation and constant values of the RMS-normalized weights can be found in Angeli and Gregory [6].

In a similar way to the LOS calculation, the WFE calculation involves creating a matrix, $C_{Z e r n}$ which premultiplies the $C$ matrix in equations 2.21 and 2.23 . The $C_{Z e r n}$ matrix transforms the z-direction translational displacements of the primary mirror into Zernike coefficients and is called a linear sensitivity matrix.

$$
z_{Z e r n}=C_{Z e r n} \mathbf{C x}
$$

where $z_{Z e r n}$ is the $N_{z} \times 1$ vector of Zernike coefficients. $C_{Z e r n}$ is the inverse of the Zernike matrix, Z,

$$
C_{Z e r n}=\mathrm{Z}^{-1}
$$

where the Zernike matrix is defined as,

$$
\underset{n \times N_{z}}{\mathrm{Z}}=\left[\begin{array}{cccc}
\vdots & \vdots & \vdots & \vdots \\
\operatorname{Zern}_{1}\left(\rho_{i}, \phi_{i}\right) & \operatorname{Zern}_{2}\left(\rho_{i}, \phi_{i}\right) & \cdots & \operatorname{Zern}_{N_{Z}}\left(\rho_{i}, \phi_{i}\right) \\
\vdots & \vdots & \vdots & \vdots
\end{array}\right]
$$

and $n$ is the number of primary mirror nodes. 3 Zern denotes the Zernike functions shown in Fig. 2-8. The radial and azimuthal stations of the $i^{\text {th }}$ primary mirror node are $\rho_{i}$ and $\phi_{i}$, respectively. This can be validated by recognizing that the primary mirror displacements can be recovered by multiplying the Zernike functions by their coefficients,

$$
\mathrm{Zz}_{\mathrm{i}}=\mathrm{Cx}
$$

\footnotetext{
${ }^{3}$ If the $\mathrm{Z}$ matrix is singular, then a pseudo inverse is used
} 


\subsubsection{Disturbance model}

The primary disturbance source in the MOST system is assumed to be imbalance of the reaction wheel assembly. The model of the imbalanced reaction wheels is represented with harmonic components. Davis et al. [23] show how the harmonic frequencies are obtained from experiments on the Hubble reaction wheels. The amplitude of the imbalance disturbance is proportional to the square of the wheel speed and is represented as

$$
m_{i j k}(t)=C_{j k} f_{i}^{2} \sin \left(2 \pi h_{j k} f_{i} t+\phi_{i j k}\right)
$$

where $m$ is the force or torque, $i$ is the wheel number, $j$ is the direction number ( 3 forces, 3 torques), $k$ is the

harmonic number, $C_{j k}$ is the amplitude coefficient, $f_{i}$ is the wheel speed of the $i^{\text {th }}$ wheel, $h_{j k}$ is the ratio of frequency of the $k^{t h}$ harmonic of the $j^{\text {th }}$ disturbance to the frequency of the wheel rotation and $\phi_{i j k}$ is the phase angle of the $k^{t h}$ harmonic of the $j^{\text {th }}$ disturbance of the $i^{\text {th }}$ wheel. To get the total disturbance in the $j^{\text {th }}$ direction for the $i^{\text {th }}$ wheel, $m$ is summed over the harmonics,

$$
\left.m_{i j}(t)=\sum_{k=1}^{n_{j}} m_{i j k}(t)\right)
$$

where $n_{j}$ is the number of harmonics for the $j^{\text {th }}$ disturbance. There are 5 wheels in the MOST model.

Gutierrez [39] shows how the local power spectral density of each wheel is derived from Eqn. 2.35] and how the PSD of each wheel can be combined together to get a $6 \times 6$ PSD matrix of the forces that a set of reaction wheels at certain orientations impart on the spacecraft. The PSDs on the diagonal of the $6 \times 6$ PSD matrix can be seen in Fig. 2-9. Each line represents a force or moment in the x-, y-, and z-directions in the spacecraft coordinate frame.

\subsubsection{Performance analysis}

The performance analysis method used in MOST is the PSD integration method. The design parameter values for the nominal case are listed in Table 2.2. The RWA PSD functions are shown in Fig. 2-9 and they represent input the disturbance forces and torques at the RWA node in Fig. 2-5. The OFM are chosen to be the RSS WFE and RMS LOS jitter. Their nominal values and the performance requirements are listed in 


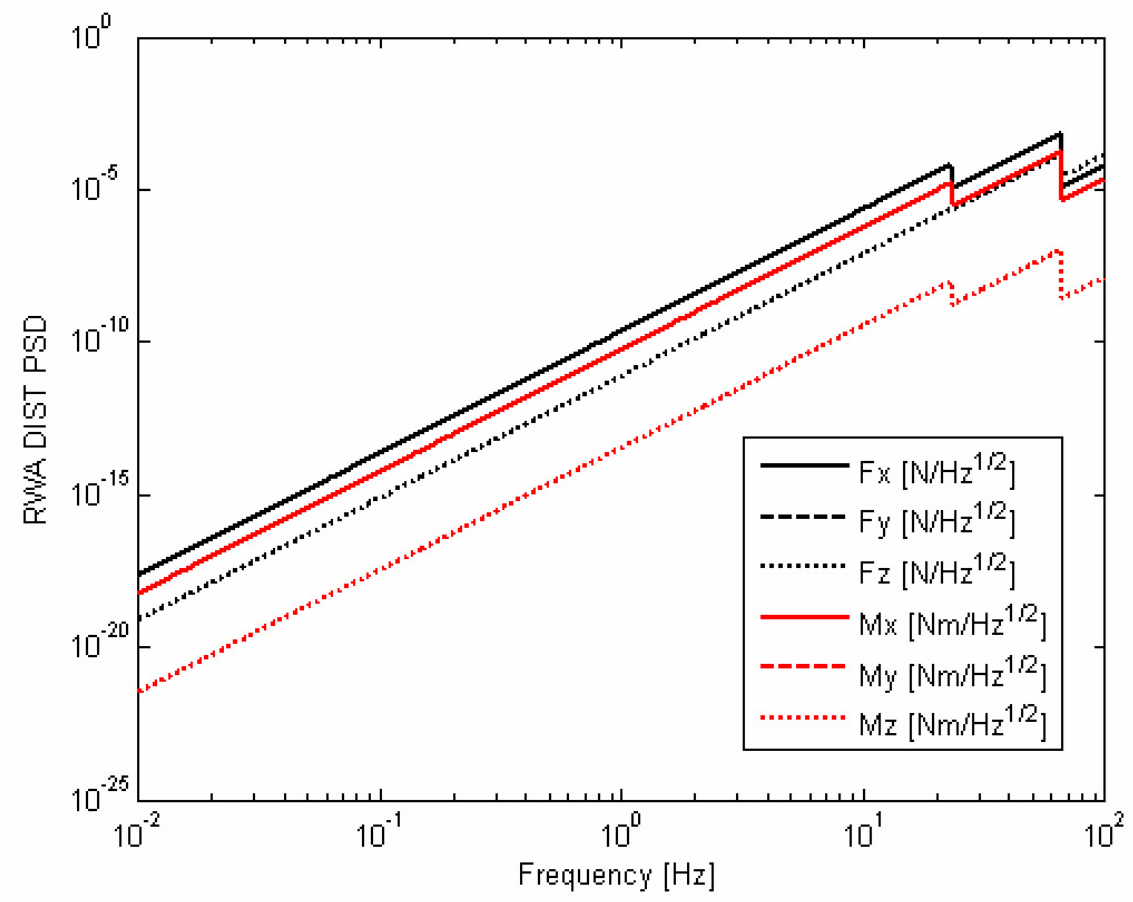

Figure 2-9. PSD of a RWA used on MOST

Table 2.3. The LOS requirement is based on diffraction-limited performance,

$$
\theta_{L O S} \leq 1.22 \frac{\lambda}{D}
$$

where $\lambda$ is the light wavelength and $D$ is the primary mirror diameter. This value is used as a $3 \sigma$ bound on LOS; dividing this value by 3 gives the $1 \sigma$ error bound [27]. The $1 \sigma$ error bound is further divided by 10 , since only $10 \%$ of the LOS error budget is allocated to the dynamic disturbances analyzed here. For a 3 $\mathrm{m}$ diameter primary mirror and $600 \mathrm{~nm}$ light wavelength, the LOS error requirement is 1.7 mas. The WFE requirement is defined as

$$
W F E \leq \frac{\lambda}{20}
$$

By treating this as a $3 \sigma$ bound and allocating $10 \%$ to the dynamic disturbance budget, the WFE requirement for MOST is $1 \mathrm{~nm}$. It is apparent that the nominal values do not meet requirements and that design changes are needed in this case. Uebelhart [77] shows how the MOST parameterized model is leveraged to find families of designs that meet requirements. 
Table 2.2. Parameter values for MOST nominal model, Fig. 2-5

\begin{tabular}{cc}
\hline \hline Parameters & Nominal values \\
\hline Mirror configuration & Monolithic \\
Mirror type & Flat \\
SST type & Tripod w/out crossbars \\
Primary mirror diameter $[\mathrm{m}]$ & 3 \\
f-number & 2 \\
Final focal ratio & 20 \\
Modal damping [\%] & 0.5 \\
RWA isolator corner frequency $[\mathrm{Hz}]$ & 10 \\
BUS isolator corner frequency $[\mathrm{Hz}]$ & 2 \\
\hline \hline
\end{tabular}

Table 2.3. OFM values for nominal MOST model, Fig. 2-5

\begin{tabular}{ccc}
\hline \hline OFM & Values & Requirements \\
\hline$L O S x[\mathrm{mas}]$ & 1.5051 & $\leq 1.7 \mathrm{mas}$ \\
$L O S y[\mathrm{mas}]$ & 3.173 & $\leq 1.7 \mathrm{mas}$ \\
$W F E_{R M S}[\mathrm{~nm}]$ & 2.9533 & $\leq 1 \mathrm{~nm}$ \\
$W F E_{R S S}[\mathrm{~nm}]$ & 14.8905 & $\leq 1 \mathrm{~nm}$ \\
\hline \hline
\end{tabular}

Figure 2-10 (a) shows both the PSD of the RSS WFE on the bottom plot and the cumulative RSS WFE on the top plot as a function of frequency. The PSD is integrated with respect to frequency to the get the cumulative RSS WFE. It can be seen that the cumulative RSS WFE converges to the nominal value of 14.8905 shown in Table 2.3. The same plot is shown in Fig. 2-10 (b) for the RMS LOS jitter OFM. It is clear that the major contribution to LOS jitter occurs between 1 and $10 \mathrm{~Hz}$ as evidenced by the peaks in the PSDs and by the steep increase in the cumulative metrics at these frequencies. This information can be used during design to change the resonant behavior of the structure or to modify the disturbance sources.

\subsection{Summary}

In this chapter the integrated modeling paradigm in which the Nyquist fidelity method will be developed is described. It is shown that the disturbance frequency bandwidth affects the system output frequency content. Furthermore, the structural model is based on the modal frequencies and modeshapes. Also, the output figures of merit are based on the frequency domain analysis and therefore reflect steady state operation performance estimates. This type of analysis relies on a stochastic model and is not appropriate for transient 

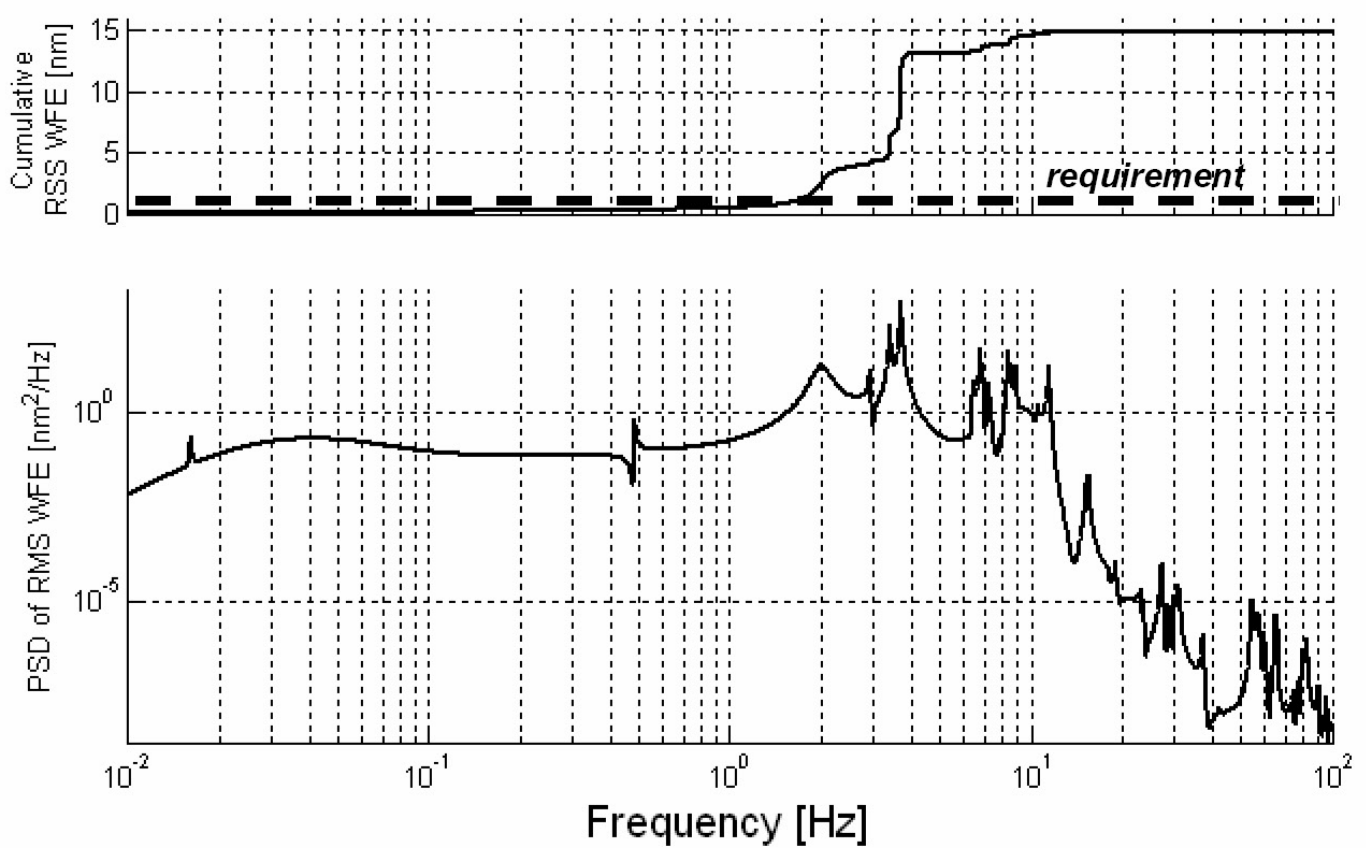

(a) Cumulative RSS WFE PSD plot
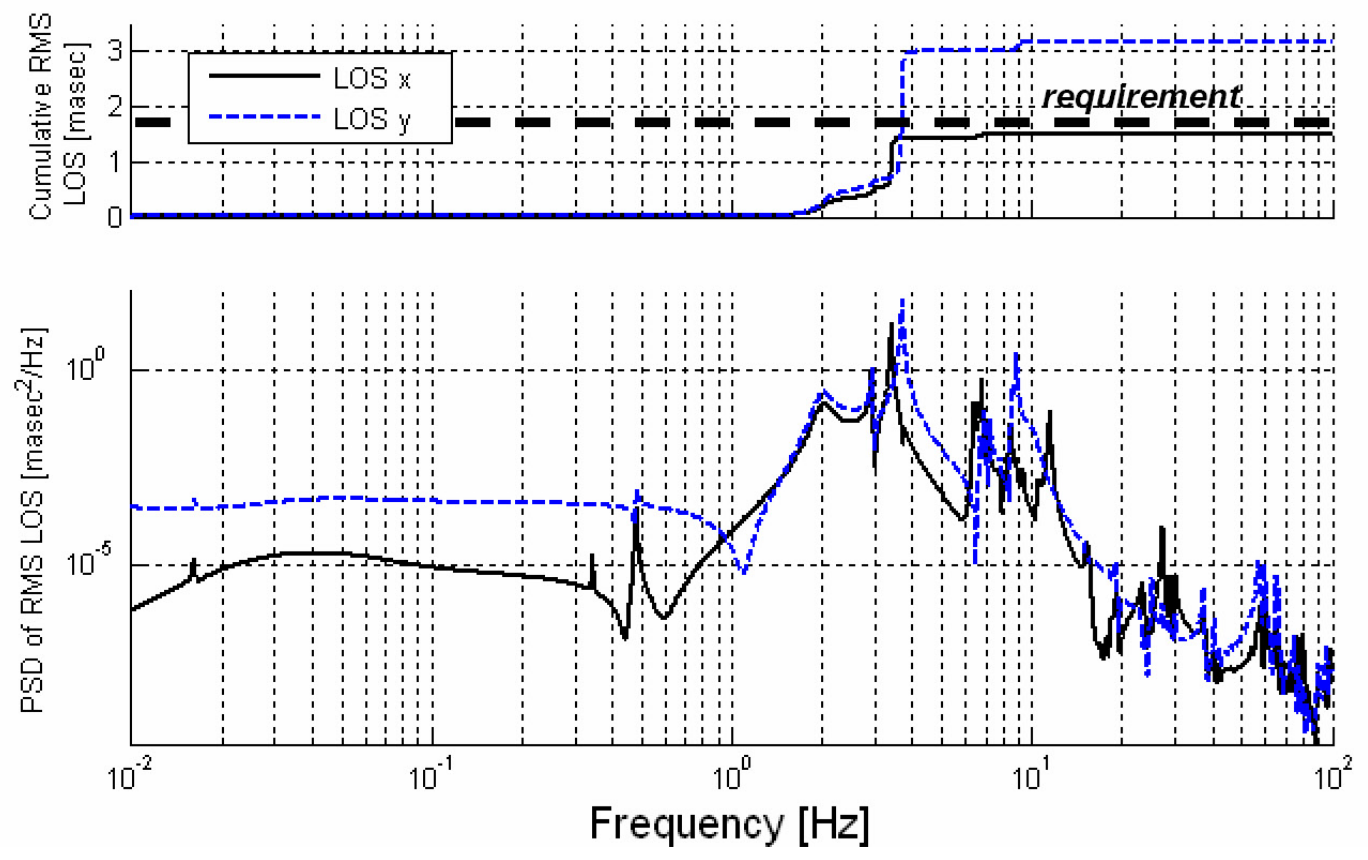

(b) Cumulative RMS LOS PSD plot

Figure 2-10. PSDs for MOST OFM 
analyses. The central case study of this thesis, MOST, is also introduced in this chapter. 


\section{Chapter 3}

\section{Nyquist Fidelity Quantification and}

\section{Assessment}

In the absence of hardware models and in the presence of new systems, fidelity quantification provides some assurance that the model, upon which important design decisions are made, contains known fidelity such that the output figures of merit have a specified degree of accuracy. Accuracy measures how close the calculation approaches the true value. In complex system modeling, fidelity has previously been defined qualitatively based on engineering judgement. In this section, fidelity is defined and quantified for a certain set of models. This quantification supplements engineering judgement during model creation, when the initial model is being built, when judging model quality, and when making design decisions.

The five main discipline models relevant to high-performance telescopes systems are structures, optics, controls, thermal, and disturbance models. Each discipline model can be modeled using a variety of techniques. Some of these are described in Chapter 2. In this thesis, the focus is on the fidelity of structural elements. In the future, these techniques can be applied to assess the fidelity of other subsystems.

In this chapter, the term fidelity is first defined. Then, the implementation method for fidelity assessment is presented. Next, fidelity is quantified for beam and plate models and the relationships between fidelity and model accuracy are established. The fidelity of the MOST nominal configuration model is then assessed. Finally, a summary of the fidelity quantification contributions is shown. 


\subsection{Definitions}

In system modeling, the term fidelity can describe how accurately a model represents the real system or the quality of an estimate produced by that model. Several other definitions of fidelity also exist. Two related terms, also heavily used in modeling and sometimes used interchangeably, are accuracy and precision. The McGraw-Hill Dictionary of Scientific and Technical Terms [69] describes theses terms as follows:

accuracy - [SCI TECH] The extent to which the results of a calculation or the readings of an instrument approach the true values of the calculated or measured quantities, and are free from error.

fidelity - [COMMUN] The degree to which a system accurately reproduces at its output the essential characteristics of the signal impressed on its input.

precision - $[\mathrm{MATH}]$ The number of digits in a decimal fraction to the right of the decimal point.

In communications, "fidelity" can indicate how faithfully a signal is reproduced. In this thesis, fidelity is defined as:

fidelity - The ability of a simulation model to accurately predict a specific output figure of merit.

Here, the term accuracy is used in the fidelity definition and is defined as above. In stochastic analysis, accuracy can describe how close the average value is to the true value. Accuracy measures the closeness of the calculated output figure of merit (OFM) value to the true value. For example, if the true value of an OFM is $10 \mathrm{~nm}$ and the calculated value, from a model, is $9 \mathrm{~nm}$, then the accuracy of the calculated value is

$$
\text { Accuracy }[\%]=100 *\left|\frac{x_{\text {calc }}-x_{\text {true }}}{x_{\text {true }}}\right|=100 *\left|\frac{9-10}{10}\right|
$$

In this example, accuracy is $10 \%$. The term precision is important as well, since computation processes used in the integrated modeling method, such as FEA, provide solutions with a limited number of significant 
figures. In evaluating the accuracy of a quantity, it is important to track how many significant figures are present in numerical calculations. Precision is discussed further in Sections 3.3 and 3.4 when evaluating modal frequency error.

This definition of fidelity will be used in this chapter to quantify fidelity using the Nyquist theorem and to describe the implementation method of assessing fidelity.

\subsection{Fidelity Quantification using the Nyquist Theorem}

In signal processing, identification of a time-domain signal is based on sampling the signal at certain discrete-time points along the waveform. A discrete Fourier transform is then performed on the sampled data to compute the spectrum (spectral components) of the waveform. The Nyquist theorem [68] states that if the sample rate captures more than two samples per wave of the highest spectral component of the signal, then the original signal can be uniquely reproduced from the sampled set using Fourier methods. This minimum sample rate is called the Nyquist rate,

$$
f_{\min }=2\left(\frac{\omega_{\max }}{2 \pi}\right)=\frac{\omega_{\max }}{\pi}
$$

where $f_{\min }$ is the Nyquist rate in samples per second and $\omega_{\max }$ is the frequency of the highest spectral component of the signal in radians per second. For a continuous signal, the Nyquist rate is defined by the continuous Fourier transform.

Another related concept is aliasing [68]. When a time-domain sample set is aliased, the original signal cannot be reconstructed properly. If the waveform contains spectral energy above half of the chosen sample rate, then those high frequencies are identified as lower frequencies in the Fourier transform. This phenomenon is called aliasing. The "aliasing frequency" is half of the sampling frequency. To avoid aliasing and reproduce the signal using sampled data, one must either sample at a rate above the Nyquist rate to capture all of the signal's spectral components or low-pass filter the signal before sampling to eliminate the high frequency components. In fact, the aliased frequencies "fold" about the aliasing frequency, so that frequencies a certain amount above the aliasing frequency will appear (after a Fourier transform) to be an equal amount below the aliasing frequency. This is called frequency folding. The concept of aliasing is used 


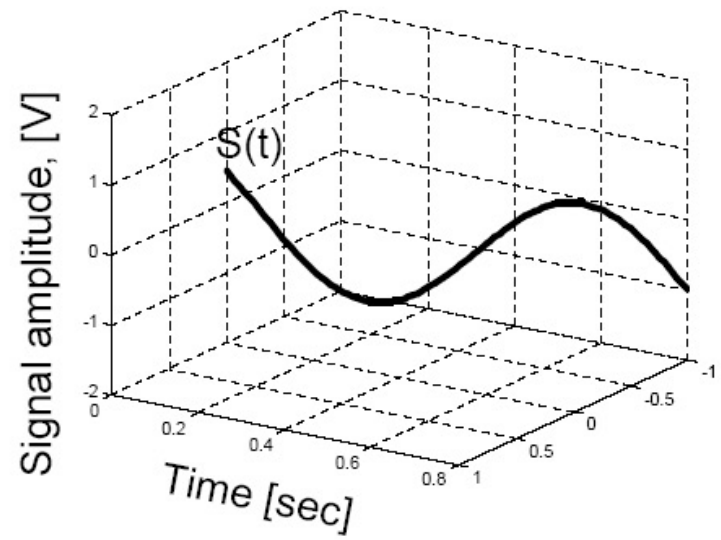

(a) Time domain signal

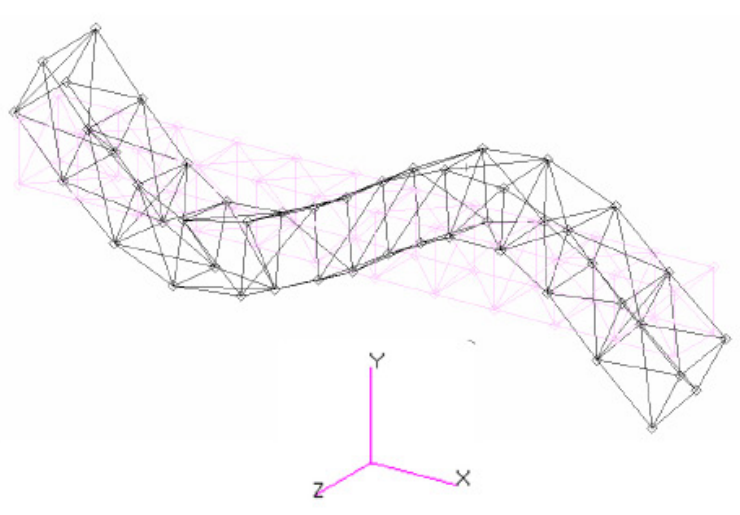

(b) Spatial wave (displacements amplified)

Figure 3-1. Time and spatial domain waves

later in this chapter when modeshapes are examined. More detailed information on signal processing theory can be found in Oppenheim, Schafer and Buck [68].

The fidelity metric for structural models is based on the Nyquist principle. The transverse deformation of a structure can be likened to a time domain signal. Figure 3-1 shows a side by side comparison of a time domain waveform, $S(t)$, and a wireframe representation of a truss structure, comprised of beams. The truss shown exhibits deflections of a resonant modeshape, which are amplified for demonstration purposes. The small square at each vertex represents a node and the lines are beam finite elements. The nodes can be likened to signal sampling points. A rule-of-thumb in modeling such structures is to apply the Nyquist theorem and to make sure that there are enough sample points (nodes) along the finite element structure in order to capture a sufficiently high spatial frequency in the model [2]. This work formalizes this rule and quantifies fidelity of structural component models based on the satisfaction of the Nyquist requirement. The fidelity metric is as follows,

$$
F_{\lambda}=\frac{n}{n_{N y}}
$$

where $n$ is the number of nodes per unit length along the dimension of a structural component and $n_{N y}$ is the number of nodes per unit length required by the Nyquist criterion ${ }^{1}$. The term, $n_{N y}$, is calculated as twice

\footnotetext{
${ }^{1}$ The subscript, $\lambda$, in Eqn. 3.2 refers to the spatial wavelength.
} 
the highest spatial frequency of interest,

$$
n_{N y}=2 \varpi_{\max }
$$

These equations show the basis of the fidelity metric. Usually in performance analyses, the highest frequency of interest is specified in the time domain with units of Hertz or radians per second. Wave propagation theory [28], [3] is used to translate this frequency into spatial frequency with units like meters per second. The definition of fidelity in Section 3.1 coincides with the quantification of fidelity here, since it captures the model's ability to accurately predict output figures if merit according to the spatial frequency of the finite element nodes. In Sections 3.3 and 3.4 the fidelity metric is explained in more detail for the beam and plate elements and the relationship between fidelity and modal frequency error is shown. The error present in the modal frequency estimates is important because the modal frequencies and modeshapes are used to construct the model of the structural system, as shown in Equations 2.21 and 2.23. The error in the model's output figure of merit, such as line-of-sight jitter, can then be estimated using sensitivity methods shown in Section 2.2.3 to relate the modal frequency error to OFM error. These techniques are further developed and described in Section 3.5 .

\subsection{Beam Fidelity}

In this section the fidelity metric for beam models is defined. The relationship between fidelity and modal frequency error in beam models is also shown. The beam models used here are finite element models of slender beams with an effectively infinite shear modulus [65]. Therefore, there is no shear deformation in the solution and the elastic axis, gravity axis, and shear center coincide.

\subsubsection{Fidelity metric definition}

A fidelity metric for a beam component is proposed which measures satisfaction of the Nyquist requirement. This metric was previously defined in Eqns. 3.2 and 3.3, where $n$ is the number of nodes per unit length along the beam length and $n_{N y}$ is the number of nodes per unit length required by the Nyquist criterion. The highest spatial frequency of interest, $\varpi_{\max }$, is related to the highest time-domain frequency, $\omega$, using 
the wave propagation equations for beams [28]

$$
\varpi=\frac{k}{2 \pi}
$$

where $\varpi$ is a spatial frequency and $k$ is the wavenumber,

$$
k= \pm\left[\frac{\omega^{2} \rho A}{E I}\right]^{1 / 4}
$$

where $\rho$ is the volumetric mass density, $A$ is the beam's cross-sectional area, $E$ is the Young's modulus and $I$ is the area moment of inertia. These equations describe the relationship between modal frequencies in the time and spatial domains. They can also provide a spatial frequency estimate based on an arbitrary time-domain frequency. It should be noted that these equations are only valid for undamped, homogenous, isotropic beams with constant cross sections. The beams in this thesis conform to these assumptions, however when these assumptions are not met, Blevins [14] provides an excellent reference for resonant behavior of various types of beams.

Substituting the time-domain frequency of interest into $\omega$ in Eqn. 3.5, the spatial frequency, $\varpi$ and the Nyquist requirement, $n_{N y}$, can be calculated using Eqns. 3.3 and 3.4 . The time-domain frequency of interest, $\omega_{\max }$, is the highest frequency component in the input disturbance frequency spectra, $S_{w w}$. Finally, the fidelity of an instantiated beam model is calculated by dividing the number of nodes per length present in the beam model, $n$, by $n_{N y}$. The value $n$ is the sampling frequency of the beam model. If the fidelity is greater than or equal to one, then the resonant beam modes are free from aliasing up to the maximum disturbance frequency, $\omega_{\max }$.

It is important to note that as in signal processing, the Nyquist theorem only holds for equally spaced nodes. Therefore, this fidelity metric also requires this assumption. Also, the sampling of the structure is only performed at the nodes. FEA, however, uses nodal interpolation functions to describe the displacements in between FE nodes. The type and order of the interpolation function depends on the finite element formulation. Therefore, the fidelity metric defined here implicitly describes the appropriateness of the FE nodal interpolation function. The beams used here contain second order displacement interpolation functions [65]. 


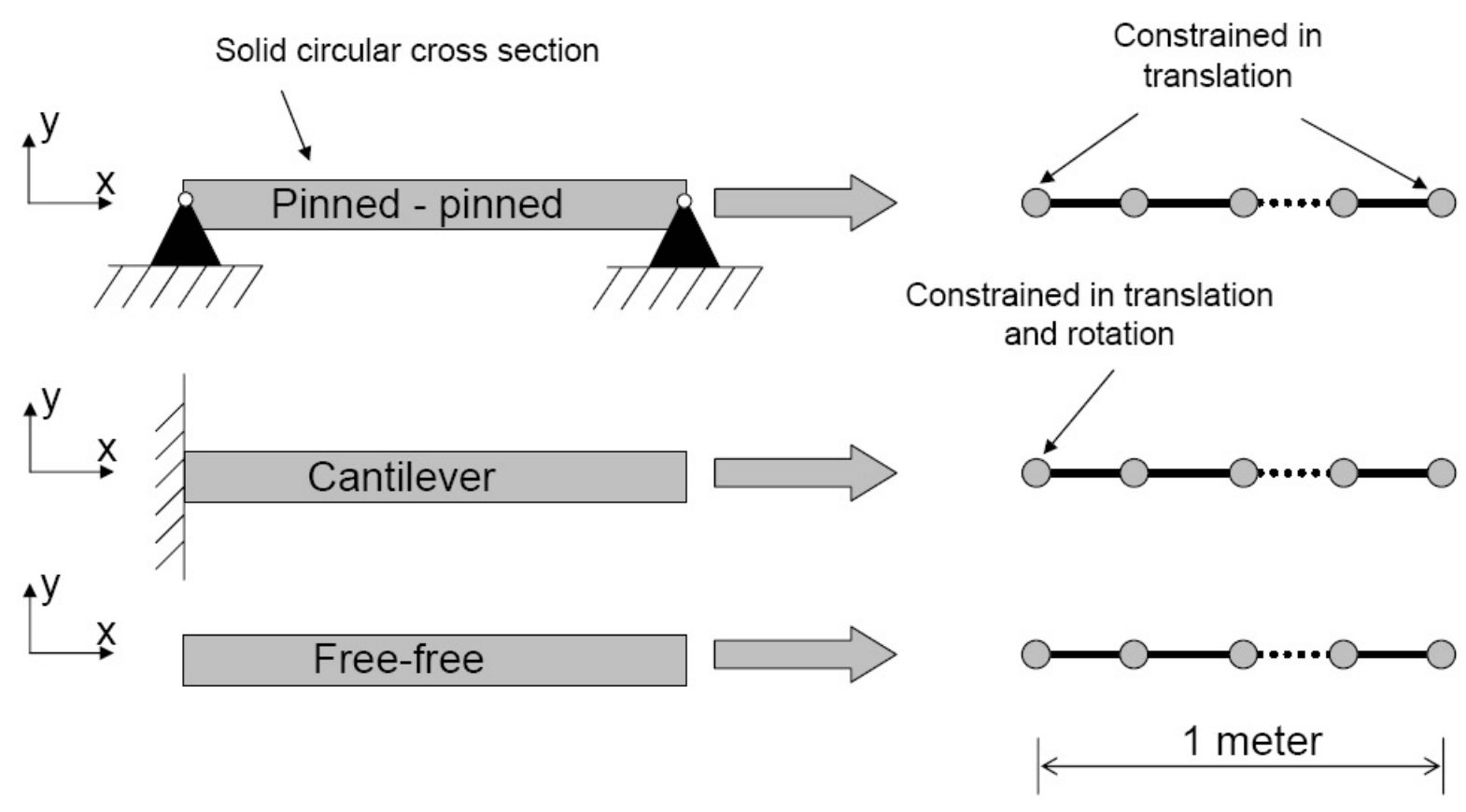

Figure 3-2. Finite element models

\subsubsection{Modal frequency accuracy relationships}

To define the relationship between fidelity and modal accuracy, several finite element beam models are created and their modal accuracy as a function of fidelity is observed.

A 2-D pinned beam, a 2-D cantilever beam, and a 2-D free-free beam, each of unit length 1, are modeled as aluminum with a solid, circular, 10 centimeter diameter cross section using simple beam elements, as shown in Fig. 3-2, In Fig. 3-2, the circles are finite element nodes, the solid lines represent beam elements, and the dotted line represents the varying number of elements in each instantiated model. Twenty models are constructed for each boundary condition, each containing the corresponding number of evenly spaced beam elements (one through twenty).

In addition to varying the number of nodes for each beam, the material and geometric properties are varied one at a time. The nominal material properties and the varied properties are shown in Table 3.1 .

First, the fidelity of the first ten modes in each beam model is calculated. The numerator of the fidelity metric, $n$, is the number of nodes per unit length present in each model. The denominator, $n_{N y}$, is twice the spatial frequency of each transverse flexural mode (of which the first ten are examined in each beam model). 
Table 3.1. Beam properties

\begin{tabular}{lcc||ccc}
\hline \hline Property & Symbol & Units & Nominal value & Variation 1 & Variation 2 \\
\hline Young's modulus & $\mathrm{E}$ & {$[\mathrm{Pa}]$} & $7 \times 10^{10}$ & $7 \times 10^{9}$ & $7 \times 10^{11}$ \\
Length & $\mathrm{L}$ & {$[\mathrm{m}]$} & 1.0 & 0.5 & 10.0 \\
Density & $\rho$ & {$\left[\mathrm{kg} / \mathrm{m}^{3}\right]$} & 2700 & 1350 & 5400 \\
Diameter & $\mathrm{d}$ & {$[\mathrm{m}]$} & 0.1 & 0.2 & 0.15 \\
\hline \hline
\end{tabular}

The spatial frequencies are obtained by first calculating the analytical time-domain frequency using the solution to the Bernoulli-Euler beam equation, shown in Appendix A Then, the time-domain frequencies are translated into spatial frequencies using Eqns. 3.5 and 3.4, yielding $\varpi_{t r u e}$.

After the fidelities are calculated, the first ten transverse flexural modes are estimated using a finite element analysis (FEA). The modal accuracy of the estimate provided by the FEM is represented as modal frequency error (MFE) in percent for each mode in each beam model and is calculated as,

$$
\mathrm{MFE}=\frac{\omega_{F E M}-\omega_{\text {true }}}{\omega_{\text {true }}} \times 100
$$

where $\omega_{\text {true }}$ is the solution to the analytical model and $\omega_{F E M}$ is the estimate of that modal frequency provided by the FEM. The analytical beam solution is shown in Appendix A. The results of this numerical experiment are shown graphically in Figs. 3-3 and 3-4. These plots contain data points for the first 10 transverse flexural modes of beam models with the varying fidelity and varying properties shown in Table 3.1 . These plots agree with previous theory; Section 4.3 in Bathe [11] shows that the finite element solution converges to the true solution of the model from above with increasing number of elements (decreasing mesh size) and a consistent mass matrix. The convergence equation derived in Bathe [11] can be written as,

$$
\left\|\mathbf{u}-\mathbf{u}_{h}\right\|_{1} \leq c h^{k}
$$

where $c$ is a constant, $h$ is the characteristic size of the finite element and $k$ is the complete polynomial order of the element's interpolation function. The terms $\mathbf{u}$ and $\mathbf{u}_{h}$ represent the true and finite element solution displacement values, respectively. The left hand side, $\left\|\mathbf{u}-\mathbf{u}_{h}\right\|_{1}$ represents the error estimate for the strains and stresses (displacement gradient), since the 1-norm is essentially the energy norm. Therefore, the order 


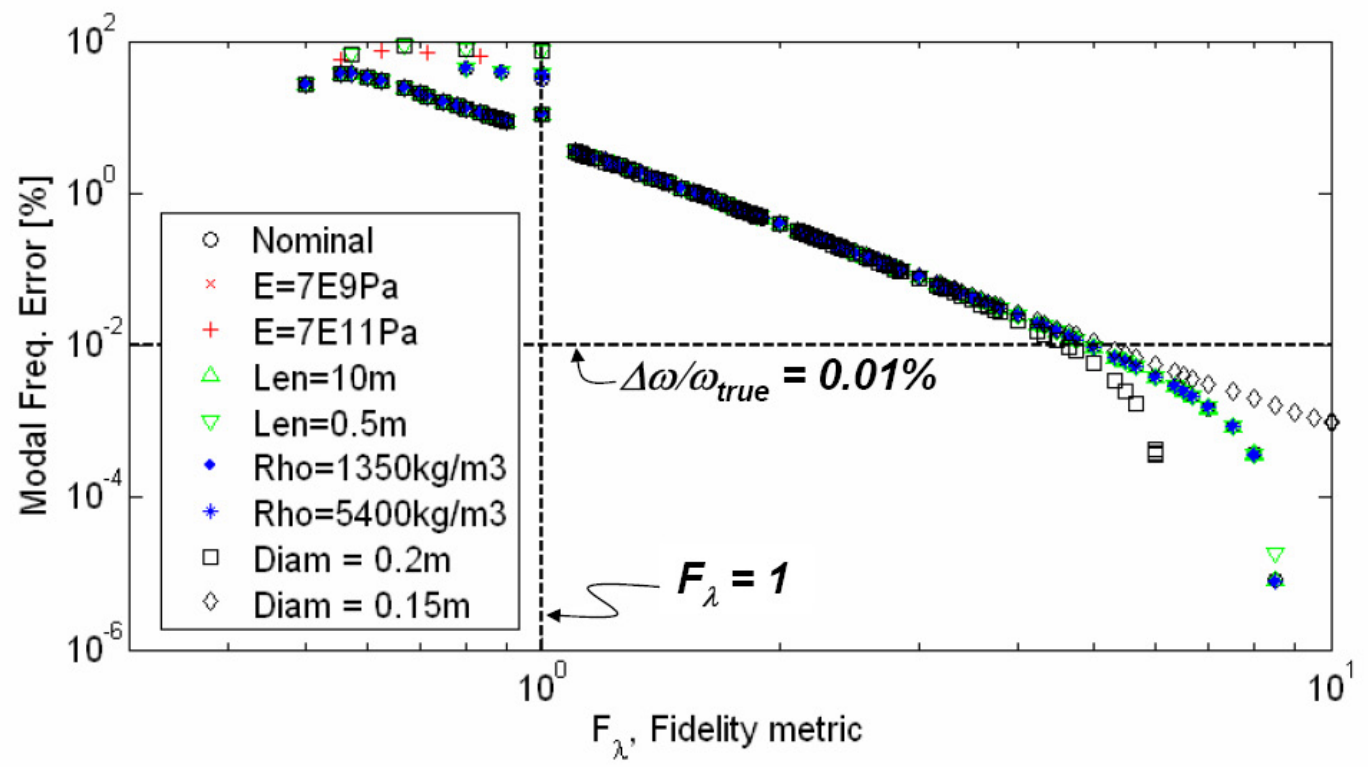

(a) Pinned beam

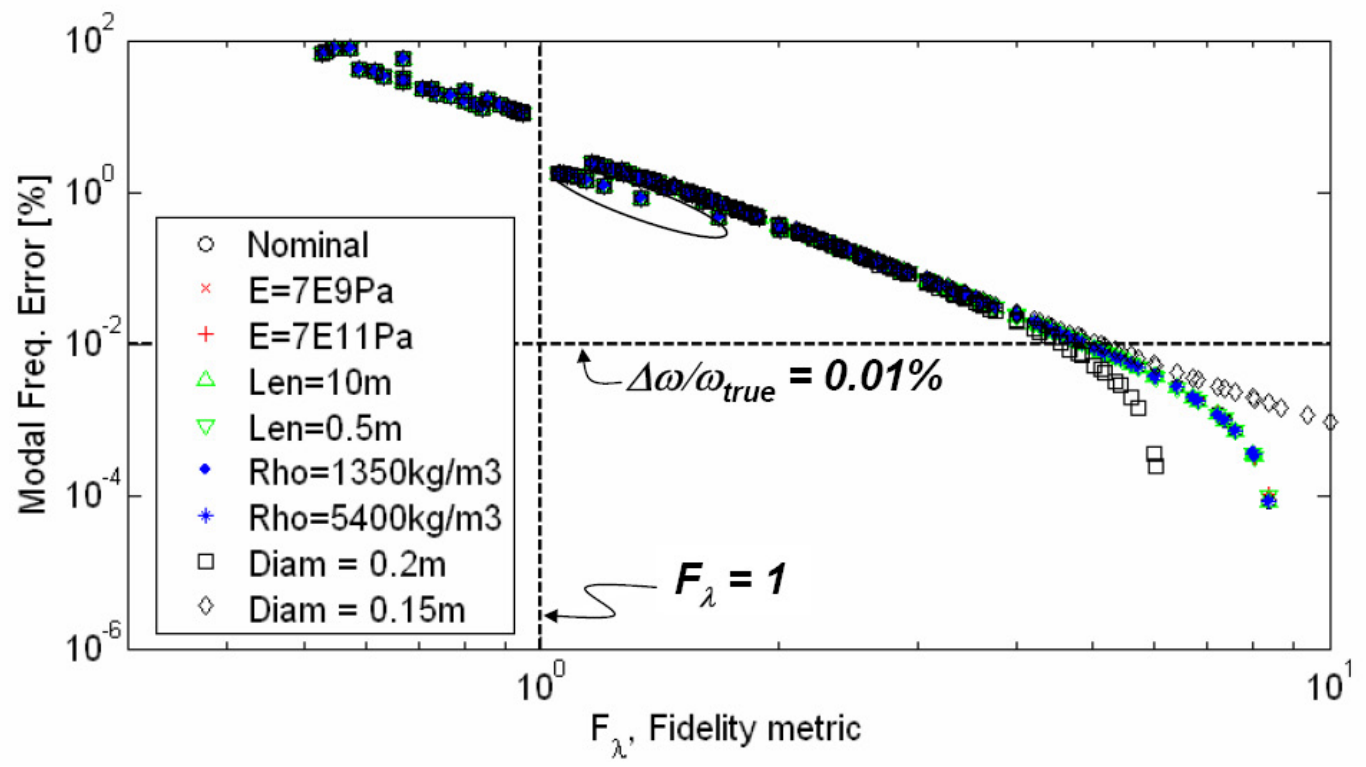

(b) Cantilever beam

Figure 3-3. Modal accuracy vs. fidelity for first 10 modes, varying properties 


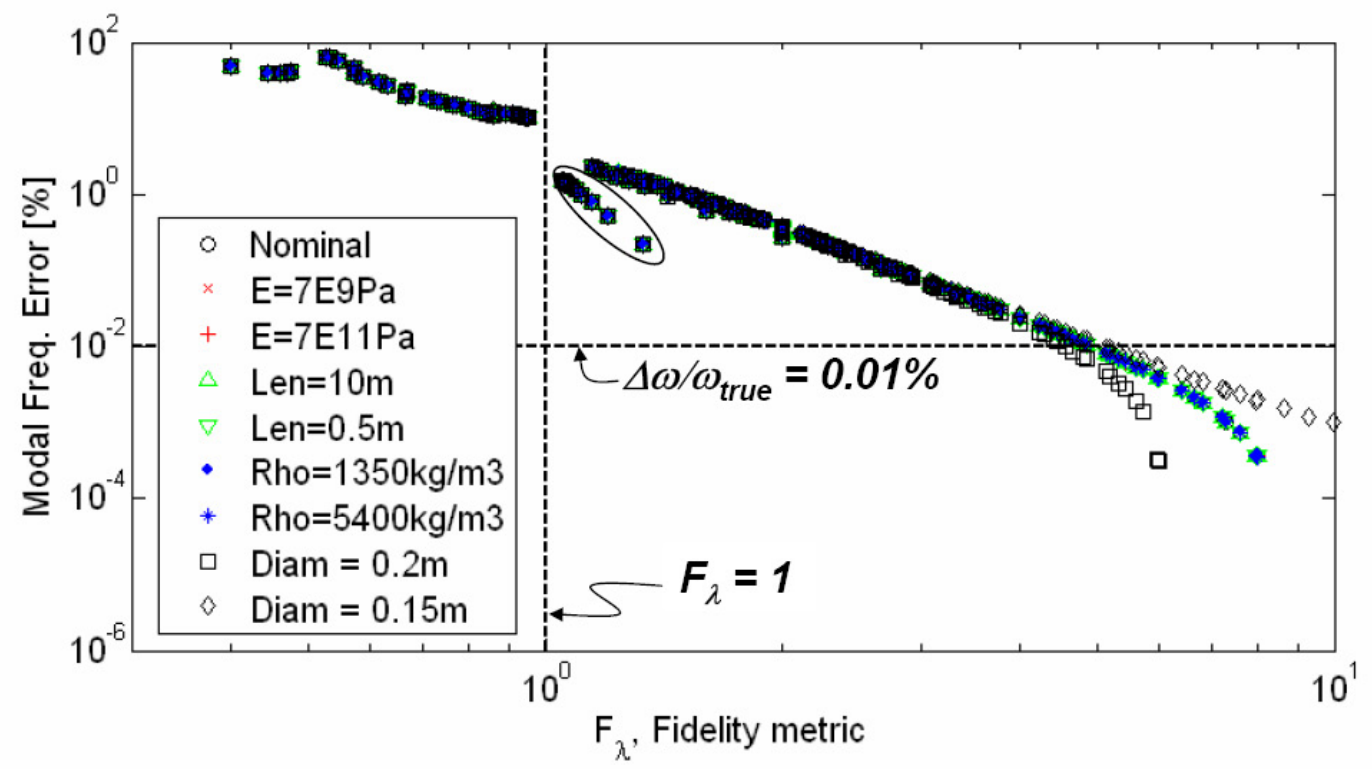

(c) Free-free beam

Figure 3-4. Modal accuracy vs. fidelity for first 10 modes, varying properties

of convergence of displacement errors is determined by $k$. Since $h$ and $c$ are positive numbers, the finite element solution converges monotonically with decreasing mesh size.

The plots in Figs. 3-3 and 3-4 show a remarkable result: the fidelity metric defined in Section 3.2 is strongly related to modal frequency error across varying properties and across modes. Therefore, the modal frequency error can be specified a priori and the corresponding fidelity level would suggest a mesh density that would achieve the desired error for all modes up to a certain frequency.

An exception to the high correlation shown by the data in these plots are the data points encircled in the cantilever and free beam cases, shown in Figs. 3-3 (b) and 3-4, respectively. These points deviate from the other data due to the evanescent wave behavior present in the cantilever and free cases. Evanescence has the effect of "stretching" the effective spatial wavelength of a mode, thereby decreasing the effective spatial frequency of the mode. This has the effect of decreasing the effective Nyquist-required spatial frequency, found in the denominator of the fidelity metric (Eqn. 3.2), and thereby increasing the effective fidelity. Therefore some modes, where the effect of evanescence is great, contain higher effective fidelities, thereby enabling lower modal frequency error. This behavior is seen in the data points that are encircled in Figs. 3-3 (b) and 3-4, which fall below the general trend. Thus the curve represented by the pinned-beam data points 
acts as a conservative bound.

Below a fidelity level of 1.0 (vertical line marked in plots), the Nyquist theorem states that the models do not contain sufficient fidelity to capture the frequencies of each mode. Below a modal frequency error of $0.01 \%$ (horizontal line in plots), there are not enough significant figures in the modal frequency estimates to know the error to this precision. This is due to the number of significant figures used in the analytical and FE solution sequences. In the FE solution, only 4 significant figures are used for the cross sectional area and moment of inertia properties. Therefore, the modal frequency error metric is precise only to the fourth significant digit, or $0.01 \%$.

A curve fit is applied to the data in Figs. 3-3 and 3-4 for each boundary condition. A power law, often present in physical systems, is fit to the data within the bounds described above. The curve fit is called the Generalized Beam Relation gives the modal frequency error, $\Delta \omega$ as a function of fidelity. It is of the form,

$$
\operatorname{MFE}[\%]=\delta \omega[\%]=10^{b}\left(\mathrm{~F}_{\lambda}\right)^{a}
$$

which can also be written as,

$$
\log _{10} \delta \omega=b+a \log _{10} \mathrm{~F}_{\lambda}
$$

Note that this relationship is only valid for fidelity levels greater than 1.0.

The results can be seen graphically in Fig. 3-5 and Table 3.2 contains the curve fit properties. It should be noted that the correlation coefficient squared, $R^{2}$, is greater than 0.99 in each case. Furthermore, the slope of the this relation, $a$, is the order of convergence and is approximately -3.86 .

This is similar to the result in Bathe [11], Section 4.3.4, which plots finite element mesh size versus error in the strain energy for a plate element. The slope in that plot was 3.91, which approximates the theoretical order of convergence of 4 according to Eqn. 3.7. In that case, the polynomial order of the element was two,

$$
k=2
$$

and the strain energy is square of the energy norm. $2^{2}$ Therefore the theoretical upper bound of the convergence order on the strain energy according to Eqn. 3.7 was 4.

\footnotetext{
${ }^{2}$ The left hand side of Eqn. 3.7 is the energy norm.
} 
Table 3.2. Curve fit coefficients for generalized beam relation in Eqn. 3.8

\begin{tabular}{c||c|c|c|c|c}
\hline \hline & $\mathrm{a}$ & 95\% Conf. Int. & $\mathrm{b}$ & 95\% Conf. Int. & $R^{2}$ \\
\hline Pinned & -3.907 & $(-3.914,-3.899)$ & 0.7579 & $(0.755,0.7608)$ & 0.9990 \\
Cantilever & -3.835 & $(-3.846,-3.823)$ & 0.7099 & $(0.7053,0.7144)$ & 0.9979 \\
Free & -3.841 & $(-3.853,-3.829)$ & 0.6987 & $(0.6938,0.7037)$ & 0.9977 \\
\hline \hline
\end{tabular}

In this plot, Fig. 3-5, fidelity is plotted against modal frequency error. The modal frequency error converges as strain energy since for a mass normalized system, the eigensolution is given as

$$
\phi_{i}^{T} K \phi_{j}=\lambda_{i} \delta_{i j}
$$

where $\phi$ is a modeshape vector, $K$ is the stiffness matrix, $\lambda_{i}$ is the $i^{\text {th }}$ modal frequency and $\delta_{i j}$ is the Kronecker delta. The left hand side represents strain energy, therefore the modal frequencies converge at the same rate as the strain energy.

The fidelity is an inverse measure of mesh size, therefore the theoretical order of convergence is multiplied by -1 . Since the interpolation order, $k$, is also 2 for the beams studied here, then the theoretical order of convergence for the data shown in Fig. 3-5 is -4 , similar to the fitted order of convergence, -3.85 . What the relation shown in Fig. 3-5 adds to the convergence analysis in Bathe is a normalization of the mesh size in the form of the fidelity metric, which is an analog to $h$ in Eqn. 3.7. This enables the value for the constant $c$, in Eqn. 3.7 to be determined for a wide range of beam properties. Now, instead of knowing only the order of convergence for beams, the error convergence relationship is defined for a general beam.

The curve fit for the pinned-pinned case is the most conservative relationship because for a specified modal frequency error, the highest level of fidelity over the three boundary conditions is indicated. Therefore these coefficients are used to define the Generalized Beam Equation as

$$
\operatorname{MFE}[\%]=\Delta \omega[\%]=10^{0.7579}\left(\mathrm{~F}_{\lambda}\right)^{-3.907}
$$




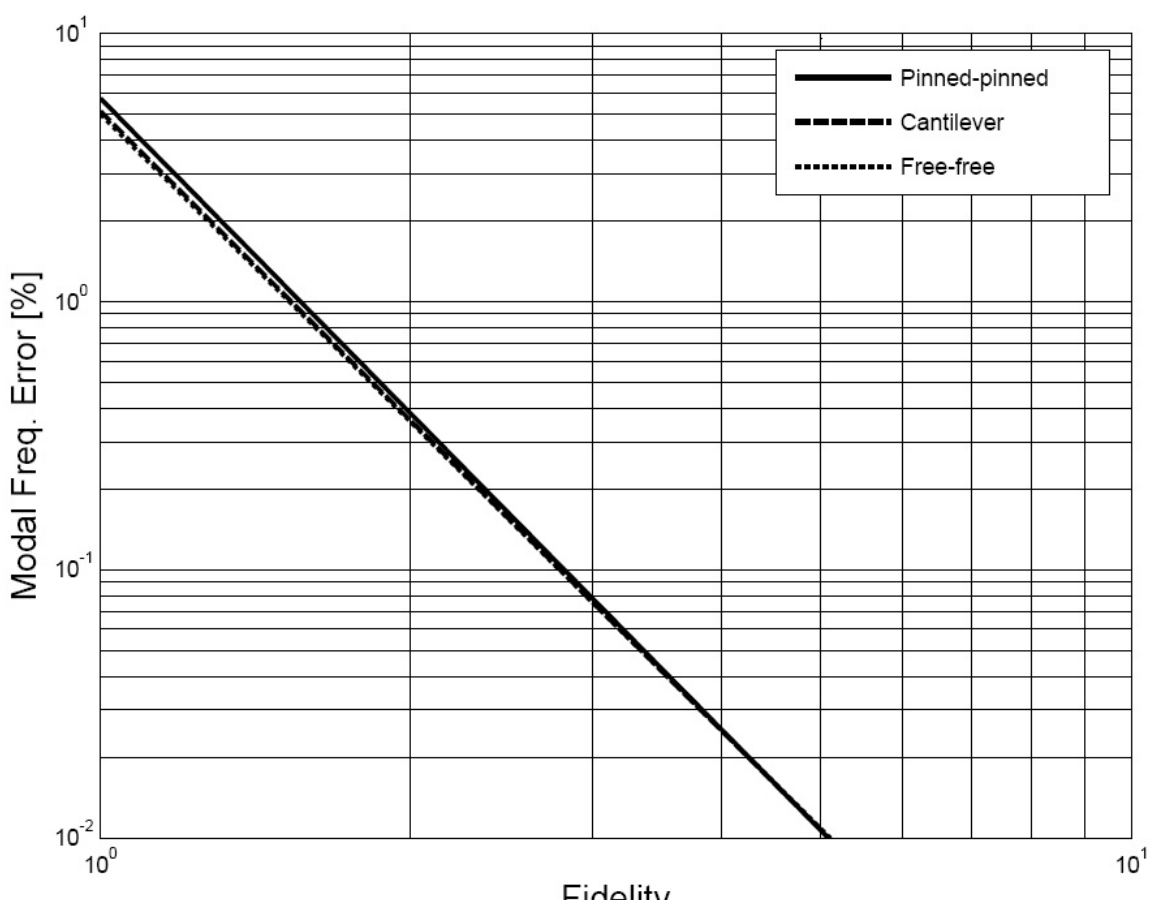

Fidelity

Figure 3-5. Power law curve fit to numerical data 


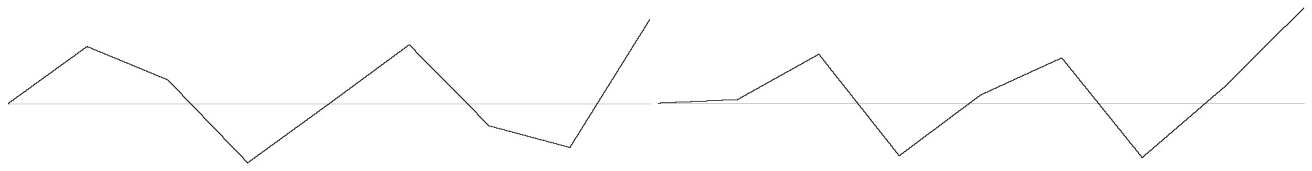

(a) 8-element cantilever beam 5th bending(b) 8-element cantilever beam 11th bending mode

mode, aliased to 5 th bending mode

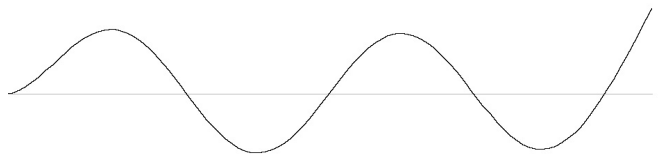

(c) 100-element cantilever beam 5th bending mode

Figure 3-6. Spatial aliasing in cantilever beam

\subsubsection{Modeshape accuracy relationships}

If there are not enough nodes in the FEM, some higher order flexural modes cannot be represented in the FEM solution, resulting in aliasing. Figures 3-6 (a) and (c) show the fifth transverse bending mode of the cantilever beam for the 8- and 100-element FEMs, respectively. Figure 3-6 (b) shows the 11th transverse bending mode. The straight horizontal line is the undeformed shape. Since there are not enough elements in the 8 -element model to represent the 11th bending mode, it is spatially aliased to the fifth bending mode.

This frequency-folding property also appears here. Figure 3-7 shows two spatially aliased modes for the free-free beam. The fifth bending mode is shown in Fig. 3-7 (a) in the 8-element free free beam. As in the cantilever case, a higher spatial frequency mode is aliased to the fifth bending mode, as shown in Fig. 3-7 (a) and (c). The 11th bending mode is also aliased to the 3rd bending mode as shown in Fig. 3-7 (b) and (d). In this model, the spatial frequencies are folded about the 7th bending mode. Higher aliased bending modes calculated in the model will alias to lower bending modes, and lower aliased bending modes will alias to higher bending modes. For example, the 8th bending mode will alias to the 6th bending mode and the 12th bending mode will alias to the 2 nd bending mode.

In addition to visual inspection, the modeshapes can be quantitatively compared using the Modal Assur- 


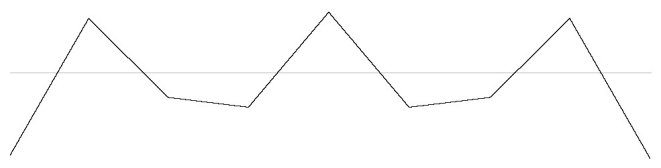

(a) 5th bending mode

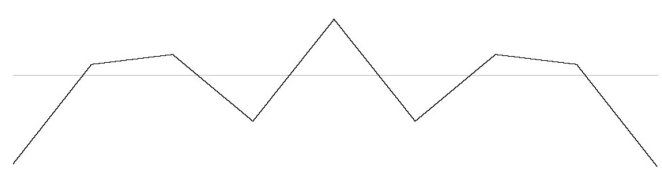

(c) 9th bending mode, aliased to 5th bending mode

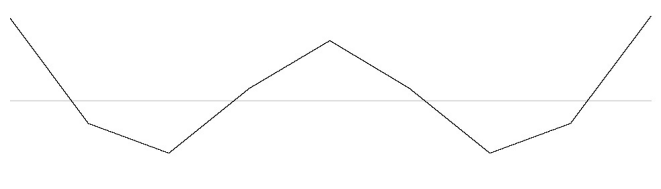

(b) 3rd bending mode

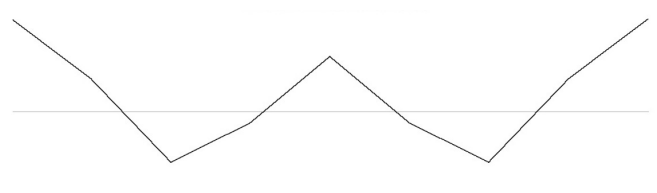

(d) 11th bending mode, aliased to 3rd bending mode

Figure 3-7. Frequency folding in an 8-element free-free beam

ance Criterion (MAC) [5],

$$
\operatorname{MAC}\left(\left[\phi_{X}\right]_{i}[\phi]_{j}\right)=\frac{\left|\left[\phi_{X}\right]_{i}^{\mathrm{T}}[\phi]_{j}\right|^{2}}{\left(\left[\phi_{X}\right]_{i}^{\mathrm{T}}\left[\phi_{X}\right]_{i}\right)\left([\phi]_{j}^{\mathrm{T}}[\phi]_{j}\right)}
$$

where the $\left[\phi_{X}\right]_{i}$ identifies the modeshape being tested and $[\phi]_{j}$ are the modeshapes of the "truth" model. This metric is usually used to compare experimental modeshapes to modeled or analytical modeshapes. The MAC can take on values between zero and one. MAC values near unity identify highly matched modes, where the modeshape vectors are co-linear. Here the MAC is used to measure the deviation of the flexural modeshapes of the one- through twenty-element FEMs compared to the 100-element truth model. Since there are an unequal number of degrees of freedom in the modeshapes being compared, these modeshape vectors are of unequal length, and Eqn. 3.13 cannot be directly applied. First, the modeshapes for the onethrough twenty-element beams are linearly interpolated, so that they are of equal length to the 100 element beam modeshapes.

In the spatial aliasing examples shown in Figs. 3-6 and 3-7, the aliased modeshapes have MAC values closer to their aliased modeshapes. Table 3.3 shows these values for the 8 -element free-free beam. The MAC values are rounded to 2 nd decimal point and the empty cells correspond to MAC values less than 1E-9. Note that the high MAC values fall either along the diagonal (matched modeshapes), or are in bold 
Table 3.3. MAC values for 8-element free-free beam vs. the analytical modeshapes

\begin{tabular}{|c||c|c|c|c|c|c|c|c|c|c|}
\hline \hline \multicolumn{1}{|c||}{$\begin{array}{c}\text { Finite element model } \\
\text { bending mode \# }\end{array}$} & \multicolumn{10}{c|}{ Analytical bending modeshapes } \\
\hline 1 & 1.00 & - & 0.00 & - & 0.00 & - & 0.00 & - & 0.00 & 0.00 \\
\hline 2 & - & 1.00 & - & 0.00 & - & 0.00 & - & 0.00 & - & 0.00 \\
\hline 3 & 0.01 & - & 0.98 & - & 0.00 & - & 0.00 & - & 0.00 & 0.00 \\
\hline 4 & - & 0.02 & - & 0.95 & - & 0.01 & - & 0.01 & - & 0.02 \\
\hline 5 & 0.05 & - & 0.02 & - & 0.88 & - & 0.01 & - & 0.04 & 0.00 \\
\hline 6 & - & 0.05 & - & 0.03 & - & 0.78 & - & 0.08 & - & 0.02 \\
\hline 7 & 0.07 & - & 0.04 & - & 0.03 & - & 0.79 & - & 0.04 & 0.00 \\
\hline 8 & - & 0.21 & - & 0.18 & - & $\mathbf{0 . 4 3}$ & - & 0.06 & - & 0.00 \\
\hline 9 & 0.23 & - & 0.20 & - & $\mathbf{0 . 4 2}$ & - & 0.06 & - & 0.07 & 0.00 \\
\hline 10 & - & 0.25 & - & $\mathbf{0 . 4 8}$ & - & 0.06 & - & 0.00 & - & 0.04 \\
\hline \hline
\end{tabular}

(aliased modeshapes). For example, the 9th bending mode in the 8-element beam is aliased to the 5th bending mode in the analytical beam, and the MAC value is 0.42 . For the non-aliased modes, the high MAC values lie along the diagonal and are matched to their correct modeshapes in the truth models. Frequency folding is also apparent here, the aliased modes are folded about the 7th bending mode in the 8-element beam.

The MAC can be evaluated for each bending mode and for each nominal FEM presented in the last section (1-20 beam elements). The plots in Fig. 3-8 show these MAC values plotted against the fidelity metric. Here, each bending mode is compared only to the corresponding bending mode in the analytical model, corresponding only to the values on the diagonal in Table 3.3 . For example, the MAC value for the 11 th bending mode in the 8 -element beam model will compare that mode to the 11th bending mode in the truth model. Therefore the aliased modes will have low values of MAC. In these figures it is clear that below the Nyquist requirement of two nodes per spatial wavelength, where the fidelity metric is unity, the MAC value is low and the modeshapes are therefore not matched. Above the Nyquist requirement, however, the MAC values are high, above 0.7 , and the modeshapes in the model match to the modeshapes in the truth model. This provides quantitative evidence that the spatial Nyquist criterion expressed in the fidelity metric is fundamental.

The presence of aliasing, frequency folding, and the clear jump in the MAC at a fidelity level of one 
further demonstrate that applying time-domain signal theory principles, such as Nyquist, to the spatial domain is a useful analogy. Furthermore, the relationship between fidelity and modal frequency error can be generalized as a power law valid across material property parameters and across three standard boundary conditions. Here only the first ten modes are shown, but the relationship has proven consistent for higher modes.

\subsection{Plate Fidelity}

The plate FE model is another component commonly used in structural conceptual design models. Plates are used in telescope system models to represent the reflective surfaces, the primary mirror, the secondary mirror, the tertiary mirror, and the solar arrays. In monolithic systems, a circular primary mirror is usually used. In segmented systems, hexagonal mirror segments are usually used. The plate fidelity will be assessed in much the same way the beam elements were analyzed, but in two dimensions.

In this section, the fidelity metrics for plates are defined two ways. First circular monolithic plates are examined, and the fidelity of these plates are defined in two directions. Next, a general plate fidelity is defined which can be applied to arbitrarily shaped plates.

\subsubsection{Circular monolithic plate}

The transverse displacement of a circular monolithic plate can be described using the plate wave equation which is given by,

$$
D \nabla^{4} w+\rho \frac{\partial^{2} w}{\partial t^{2}}=0
$$

where $D$ is the flexural rigidity, $w$ is the transverse displacement, $\rho$ is the mass density, and $t$ is time. In the homogenous (unforced) case, the resonant modes and frequencies are given by the equations in Appendix $B$. This is called classical plate theory and ignores shear deformation and the effects of rotary inertia [59]. The resonance equations consist of Bessel and trigonometric functions. Closed form solutions to the plate equation are only available for a limited number of plate shapes and boundary conditions [76].

Leissa [48] presents a numerical solution to the classical plate equation for homogenous plates. Given the material properties of the plate, the resonant frequencies and modeshapes can be calculated using values 


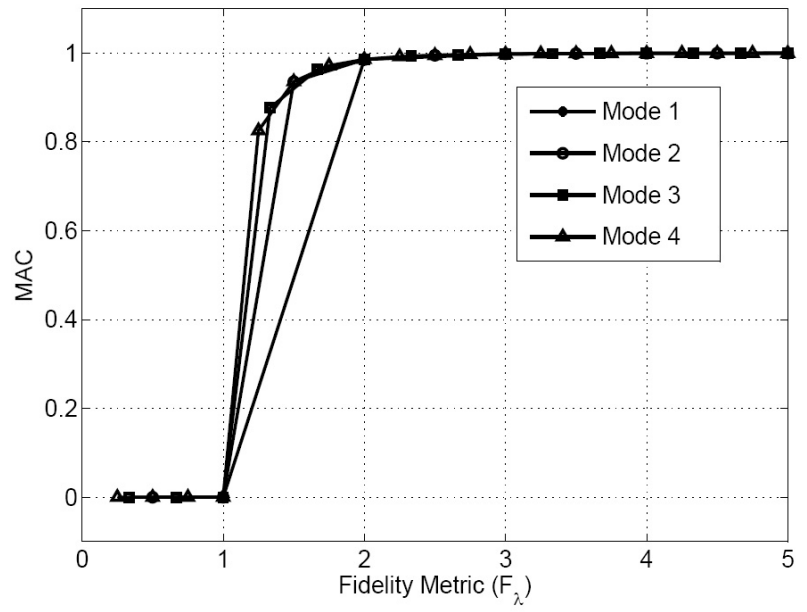

(a) Pinned beam

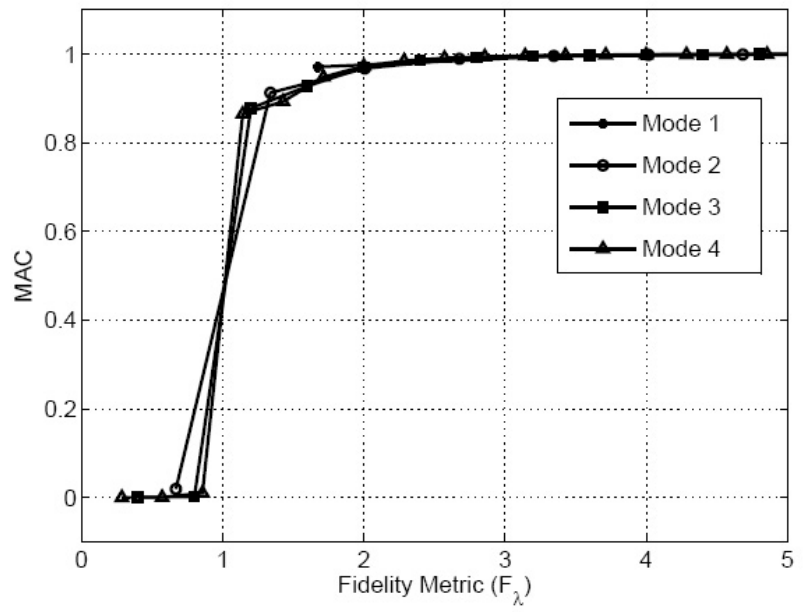

(b) Cantilever beam

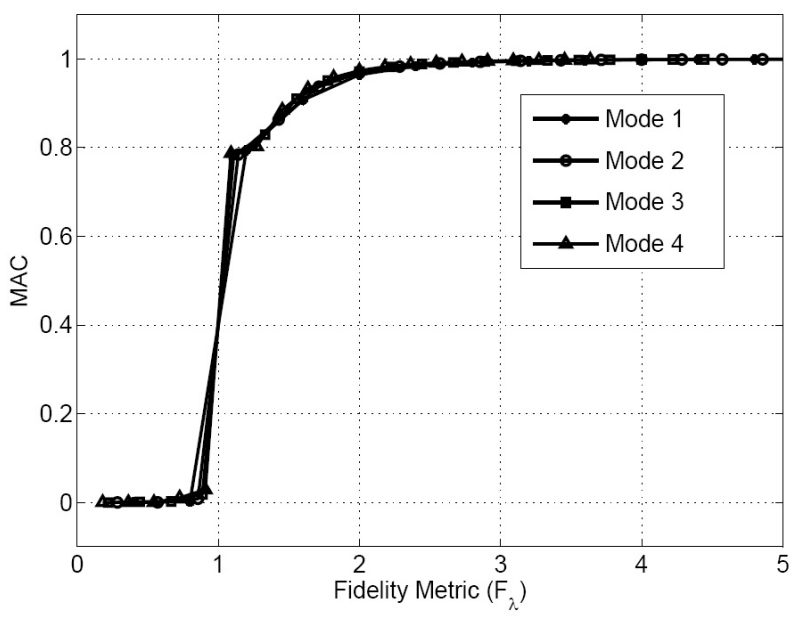

(c) Free-free beam

Figure 3-8. MAC values for beams 
tabulated in Leissa. These solutions are the "truth" models in this section.

Circular plate modeshapes contain spatial frequencies in the azimuthal and radial directions. The first four resonant modeshapes are shown in Fig. 3-9. It can be seen that modes 1 and 3 contain purely azimuthal behavior since there is no radial behavior in these modes. The lines where the deformed plate shapes intersect the undeformed shapes (shown in gray) in purely azimuthal modes are called nodal diameters. Modes 1 and 3 have two and three nodal diameters, respectively, and the corresponding azimuthal spatial frequencies, $\varpi_{\theta}$, are calculated using

$$
\varpi_{\theta}=n_{d}[\mathrm{rad} / \mathrm{rad}]
$$

where $n_{d}$ is the number of nodal diameters. This is verified by visual inspection of Fig. 3-9 (a) and (c).

By contrast, mode 2 contains purely radial behavior. Similar to the purely azimuthal modes, the purely radial modes have nodal circles which are lines where the undeformed and deformed shapes intersect. Its radial spatial frequency depends on the radius of the nodal circle, $r$, and can be estimated as

$$
\varpi_{r}=\frac{\pi}{2 r}[\mathrm{rad} / \mathrm{m}]
$$

Since this is a free-free plate, evanescent behavior is present in the radial modeshapes, shown as a hyperbolic function in the modeshape equation in Appendix B. Therefore, the radial spatial frequency shown above, $\varpi_{r}$ is an estimate. Furthermore, for higher order modes with radial frequencies, the number of nodal circles, $n_{c}$, increases, and the nodal circles lie at different, non-uniform radii. If $r$ in Eqn 3.16 is taken to be either the distance between the center and the first nodal ring or the distances between nodal circles, several frequency estimates are possible. Since the Nyquist requirement, $\varpi$ is based on the highest frequency component present in the signal, the radial spatial modeshape frequency used here is the highest of these frequency estimates. Mode 4 is a combination mode consisting of one nodal diameter and one nodal circle. Table 3.4 shows the number of nodal diameters and circles for the first 10 modes.

\section{Circular plate fidelity metrics}

Since the plate is two-dimensional, the Nyquist theorem is simply applied along two dimensions. This has an analogy in 2-D signal processing whereby an image is sampled in orthogonal directions via pixels. The 
Table 3.4. Number of nodal diameters and circles for a circular plate

\begin{tabular}{|r||c|c|c|c|c|c|c|c|c|c|}
\hline \hline Mode No. & 1 & 2 & 3 & 4 & 5 & 6 & 7 & 8 & 9 & 10 \\
\hline No. nodal diameters & 2 & 0 & 3 & 1 & 4 & 5 & 2 & 0 & 6 & 3 \\
\hline No. nodal circles & 0 & 1 & 0 & 1 & 0 & 0 & 1 & 2 & 0 & 1 \\
\hline \hline
\end{tabular}

Whitaker-Shannon sampling theorem states that the sampling frequency in two dimensions should be at least twice that of the highest frequency content in the signal in both dimensions in order to faithfully reconstruct the sampled signal [35],

$$
X \leq \frac{1}{2 B_{X}} \quad \text { and } \quad Y \leq \frac{1}{2 B_{Y}}
$$

where $X$ and $Y$ are the sampling intervals in the $x$ and $y$ directions, respectively, and $B_{X}$ and $B_{Y}$ are the maximum frequencies of the original signal in each direction. This is essentially a two-dimensional version of the Nyquist theorem and requires that the sampling be done in a regular rectangular fashion. The application of Whitaker-Shannon is ideal for image analysis or other rectangular two-dimensional forms where the gridding is rectangular. However, it is not suitable for circular plates due to the non-rectangular gridding shown in Fig. 3-10 or 3-14.

For the circular plate, the 2-D surface manifests behavior in the azimuthal and radial directions, therefore the fidelity metrics measure the satisfaction of the Nyquist requirement each of in those directions. The fidelity metrics are,

$$
F_{\theta}=\frac{n_{\theta}}{\varpi_{N y, \theta}}
$$

and

$$
F_{r}=\frac{n_{r}}{\varpi_{N y, r}}
$$

As in the beam fidelity metric, $n_{\theta}$ and $n_{r}$ are the sampling frequencies of the model being investigated. However, unlike the beam fidelity, these are in the azimuthal and radial directions, respectively. The variables $\varpi_{N y, \theta}$ and $\varpi_{N y, r}$ represent the required Nyquist spatial frequency, which is twice the highest spatial frequency present in the mode being investigated. 


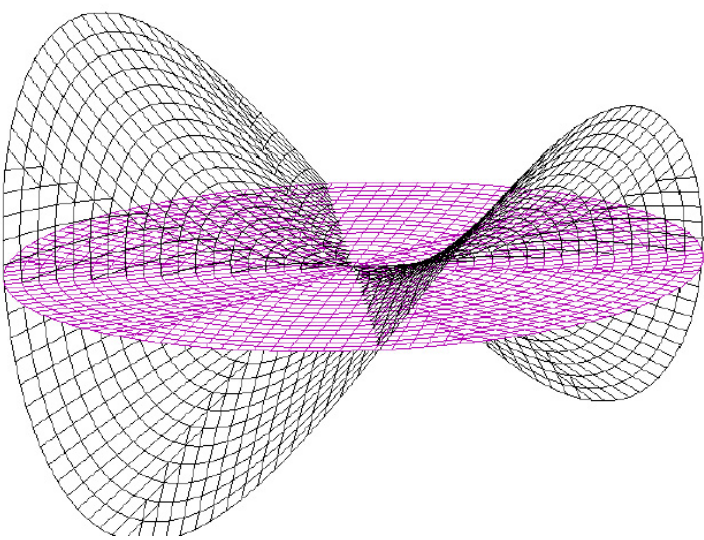

(a) Mode 1: $n_{d}=2$

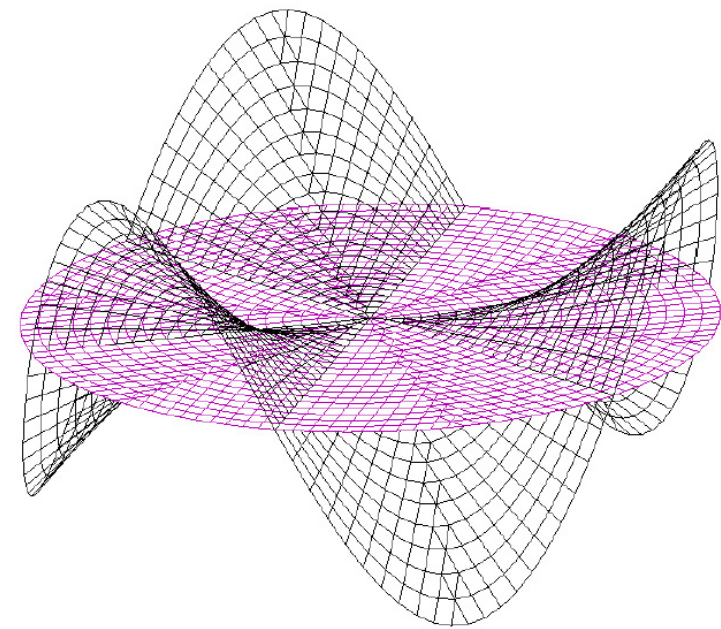

(c) Mode 3: $n_{d}=2$

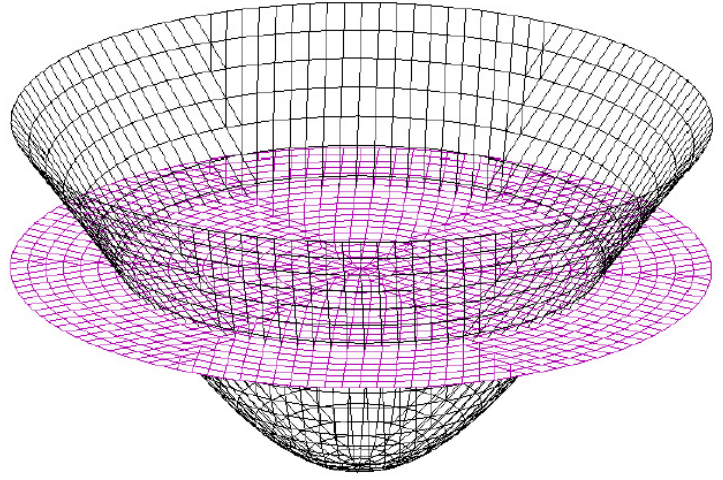

(b) Mode 2: $n_{c}=1$

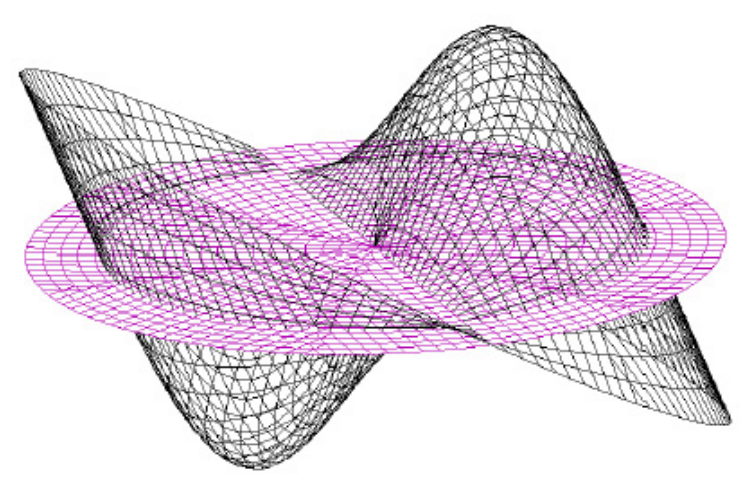

(d) Mode 4: $n_{d}=1, n_{c}=1$

Figure 3-9. First four circular plate modeshapes 


\section{Modal frequency accuracy relationships}

Similar to the method in the beam fidelity section, the relationships between the plate fidelities and the modal frequency errors are identified using numerical experiments. A one-meter diameter circular plate model is meshed using the FE layout shown in Fig. 3-10. The mesh is created using simple triangular and quadrilateral thin-plate finite elements. The mesh density can be varied by adjusting the number of element rings and the number of diameters. Figure 3-10 contains 5 rings and 5 diameters. Meshes from 1 to 20 rings and from 2 to 20 diameters are investigated. The modal frequency error is calculated by comparing the modal frequency of each model to the modal frequency given by Leissa. The solution to the classical plate equation is used since the shear effects and rotary inertias are ignored in this thin plate finite element. The fidelity metric is calculated for each of the first ten modes in each model in the azimuthal and radial directions.

The fidelity results for the purely azimuthal and purely radial modes can be seen in Fig. 3-11. Modes 1 , 3, 5, and 9 are purely azimuthal, and in Fig. 3-11 a), it can be seen that their fidelity relationships have the same shape and lie roughly along the same line. The line for mode 1 provides a conservative boundary. The purely radial modes, modes 2 and 8, shown in Fig. 3-11(b), also fall roughly along the same line.

Since the combination modes, modes 4,7 , and 10, contain both azimuthal and radial frequencies, their fidelity relationships are presented as the contour plots in Fig. 3-12(a)-(c). Each contour line represents the modal frequency percent error and is plotted against the azimuthal and radial fidelity metrics. Modal frequency error percent levels of $10 \%, 5 \%, 3 \%, 2 \%, 1 \%$, and $0.5 \%$ are shown. Each plot is cut off at a certain fidelity level where modeshapes become aliased. This level is 0.9 and is determined using the MAC and is discussed in the next section. Also shown on the combination mode fidelity plots are the "knee" points, shown as circles on each contour line. The knee points represent the minimum fidelity effort required to achieve that modal frequency error. This assumes that the modeling effort required to increase the fidelity by a certain amount is the same in the azimuthal and radial directions. On the plots, the knee points can be found by finding the point on each contour that is closest to the origin. Fig. 3-12 d) connects those knee points for each combination mode (solid lines) and for each frequency percent error (dashed lines). Using the plots in Figs. 3-11 and 3-12, the required fidelity levels in the azimuthal and/or radial directions can be determined given a desired modal frequency error. 


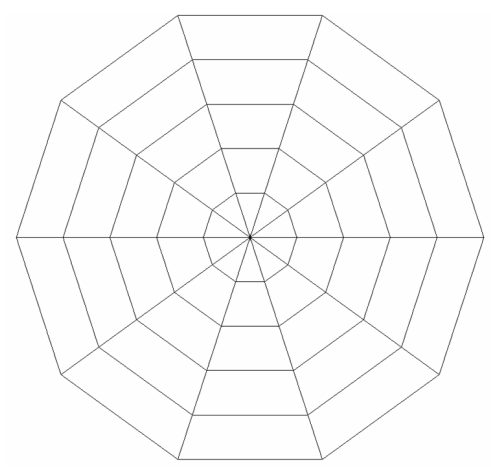

Figure 3-10. Plate finite element layout

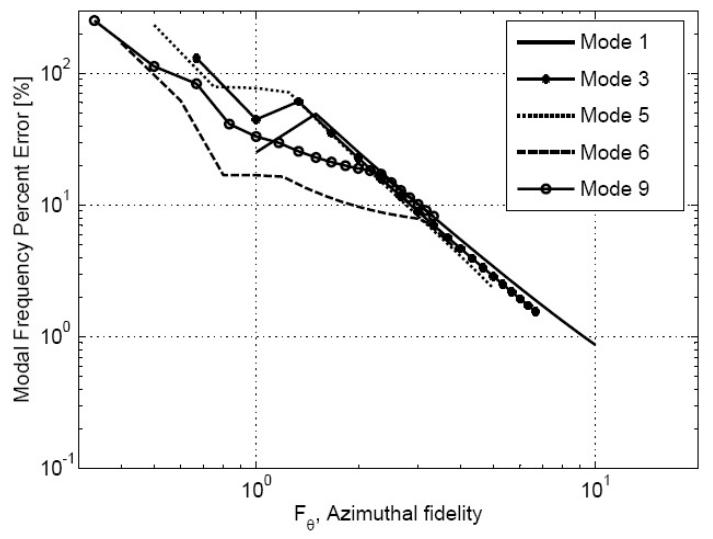

(a) Azimuthal modes

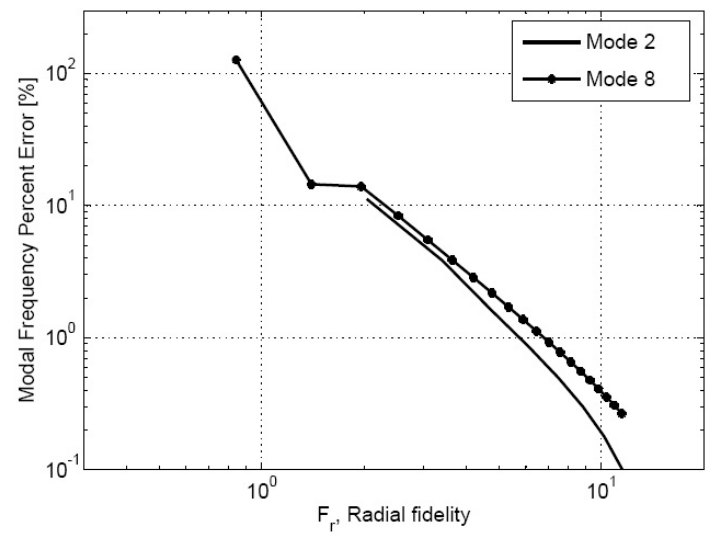

(b) Radial modes

Figure 3-11. Fidelity relationships for circular plate modes 


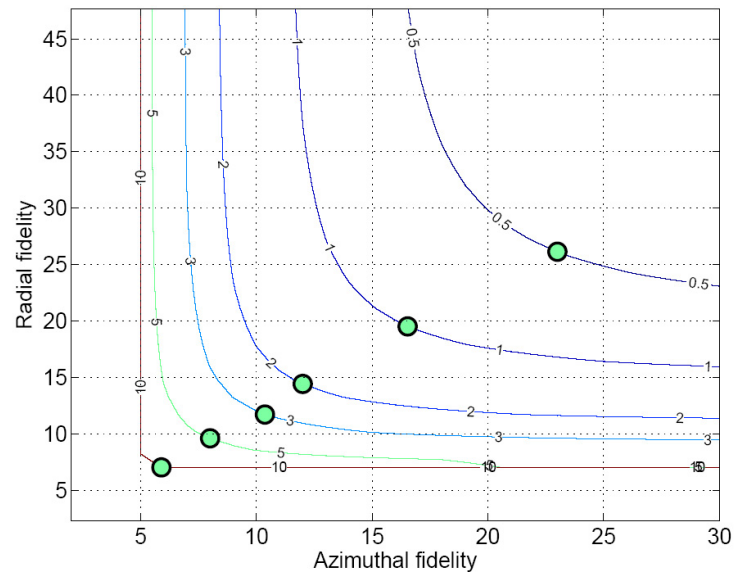

(a) Mode 4

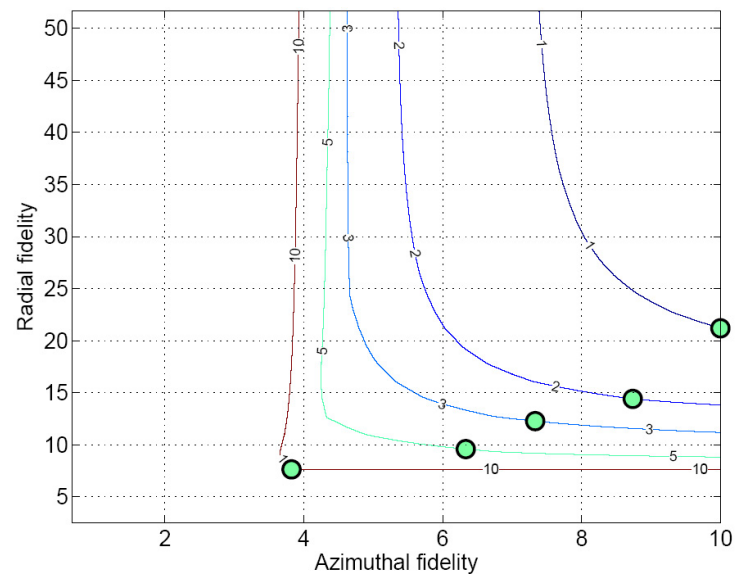

(c) Mode 10

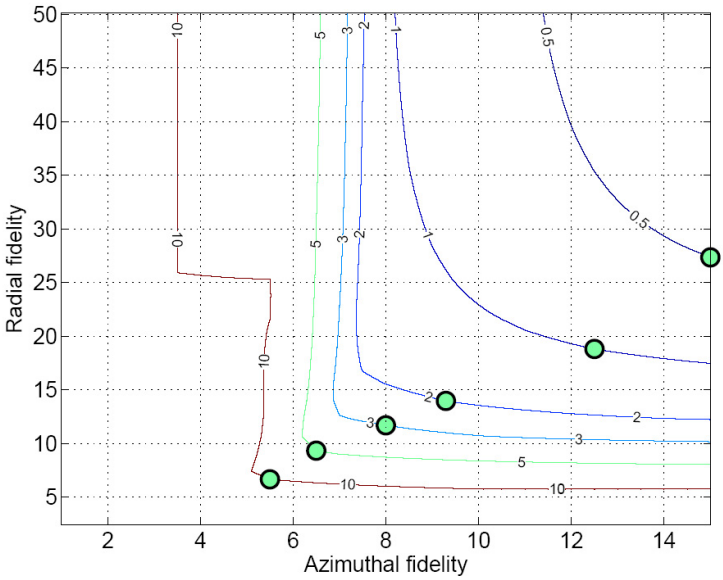

(b) Mode 7

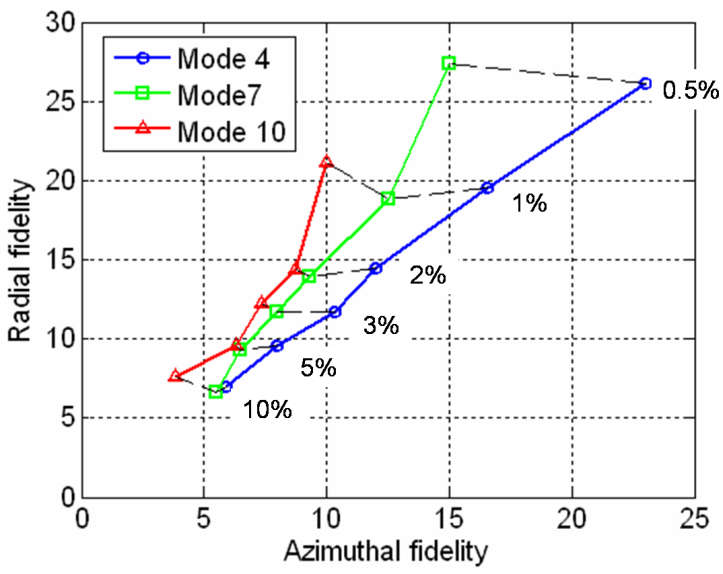

(d) Contour plot

Figure 3-12. Fidelity relationships for circular plate combination modes 


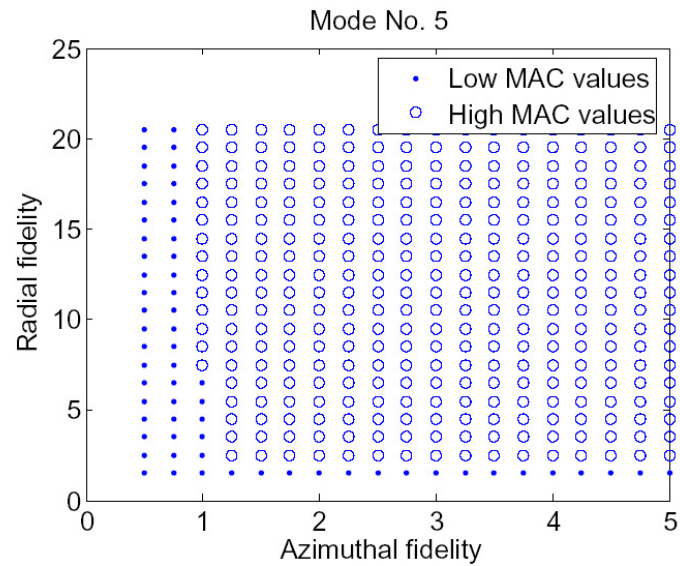

(a) Mode 5

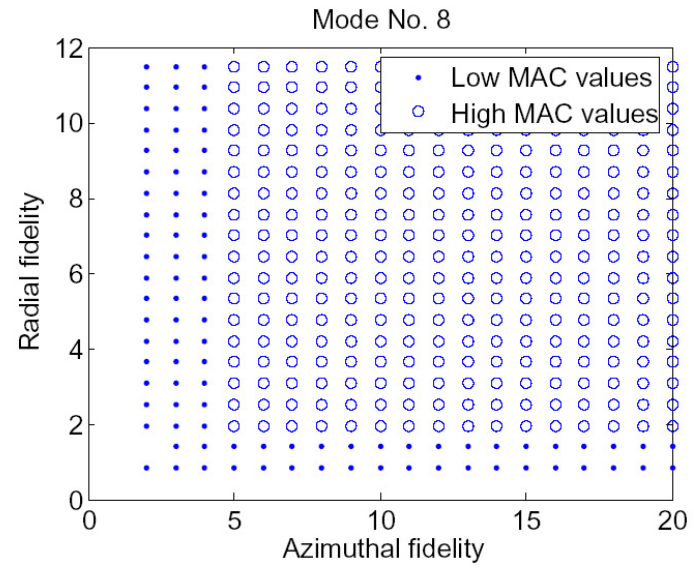

(b) Mode 8

Figure 3-13. MAC values for two modes. Low: MAC ; 0.1, High: MAC i 0.9.

\section{Modeshape accuracy relationships}

As in the beam case, there is a minimum fidelity required in each direction for all modes in order to avoid aliasing. In the beam case, this minimum fidelity level is found to be unity using the MAC to match modeshapes. This is confirmed via the Nyquist analogy which indicates aliasing would occur below a fidelity level of one. In the case of a 2D plate shown in Fig 3-10, however, the sizes of the finite elements are not uniform. By evaluating the MAC for each mode and model, the minimum fidelity required in the azimuthal and radial directions can be determined experimentally for each mode. The modeshapes that are compared using the MAC metric, Eqn. 3.13, are of unequal length, therefore the shorter modeshape, i.e. the modeshape being tested, is interpolated using the flat plate interpolation function from Matlab [58] to be of equal length to the longer, or "true" modeshape. Fig. 3-13 shows the MAC plots of Modes 5 and 8 . The dots represent MAC values less than 0.1 and circles are MAC values greater than 0.9. Empty spots indicate MAC values in between 0.1 and 0.9 . For mode 5, the minimum fidelity level is approximately 1.25 and 2.0 for the azimuthal and radial fidelities, respectively. While for mode 8, the minimum fidelities are 5.0 azimuthal and 2.0 radial. The full set of modal MAC plots can be seen in Appendix B. Fig. B-1 


\subsubsection{General plate}

The plates used in FE models of telescope systems are not always circular, nor can they always be approximated with the free-free boundary condition assumed in the last section. This section addresses plates that have an arbitrary shape. A generalized fidelity metric is presented and a fidelity relation with respect to modal frequency error is characterized for circular hexagonal plates.

\section{Generalized plate fidelity metric}

The fidelity metric for the general plate is based on the propagation of a transverse flexural wave through a thin plate. While wave propagation methods can be used to measure the speed and the phase of the wave through a structure undergoing a force, they can also be used for spectral analysis of the wave [28]. The spatial frequency of a transverse wave can be represented using the wavenumber, $k$ as,

$$
\varpi=\frac{k}{2 \pi}[\text { cycles } / m]
$$

For undamped plates, the wave motion can be described using a wavenumber of,

$$
k=\sqrt{\omega}\left[\frac{\rho h}{D}\right]^{1 / 4}
$$

where $\omega$ is the time-domain frequency, $\rho$ is the volumetric mass density, $h$ is the plate thickness and $D$ is the flexural rigidity

$$
D=\frac{E h^{3}}{12\left(1-\nu^{2}\right)}
$$

where $E$ is the Young's modulus and $\nu$ is the Poisson's ratio. In this way, an estimate of the spatial frequency present in each mode, $\varpi$, is obtained for a given modal frequency, $\omega$, geometry, and material properties. The reader is referred to Doyle [28] for a structural wave propagation reference.

Once this characteristic spatial frequency is calculated for each mode, the required Nyquist spatial frequency is simply

$$
\varpi_{N y}=2 \varpi
$$


This is used as the denominator of the generalized plate fidelity metric.

The generalized plate fidelity metric is defined as

$$
F_{\lambda}=\frac{\left(\sqrt{\# \text { nodes } / m^{2}}\right)_{F E M}}{\varpi_{N y}}
$$

As in the previous fidelity metrics, the numerator represents the nodal spatial frequency of the FEM investigated. In this case, since the nodal density is $2 \mathrm{D}$ and the denominator is $1 \mathrm{D}$, the square root of the nodal density is used as an approximation to the 1D nodal density. This assumes that the FE mesh is uniform and all the elements are the same (or close to the same) size, as in Fig. 3-14.

\section{Modal frequency and modeshape accuracy relationships}

Two different plates with two FE meshes are examined in this section. They can be seen in Fig. 3-14 A convenient way to mesh a circular plate is with a combination of quadrilateral and triangular plate elements in concentric rings as shown in Fig. 3-14 (a) which consists of 5 rings of similarly-sized elements. A hexagonal plate can be meshed with uniform sized triangular elements as in Fig. 3-14 (b), where there are 6 element rings. In the same way as the beam and plate fidelity metrics, the mesh densities of these plates are varied, thereby varying the fidelity, and the modal frequency error is observed. Both plates are evaluated with free and pinned boundary conditions. In addition, the accuracy of the modeshapes are also evaluated in this section.

In the circular plate case, the number of element rings is varied from 1 to 20 , with the modal frequency error versus fidelity results shown in Figs. 3-15 (a) and Fig. 3-16 (a) for both the pinned and free boundary conditions. The modal frequency error is calculated by taking a highly meshed model (50 element rings) as the "truth" model. The first ten modes are shown and the data points belonging to each mode are connected with a line. Since there are double modes present, there are more than 10 lines in this plot. The boundary lines at fidelity of 1 and a frequency error of $0.01 \%$ are also shown. The data in this plot seem to fall roughly along the same line and have the same slope within these boundary lines. A least squares linear 


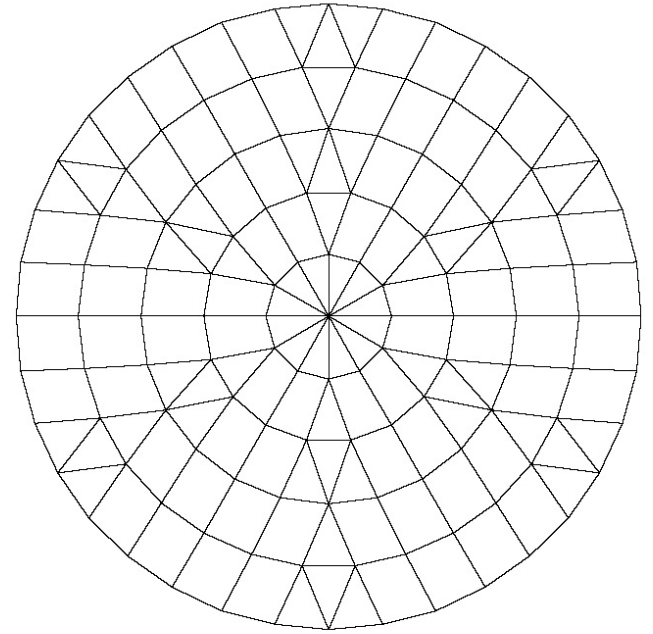

(a) Circular plate

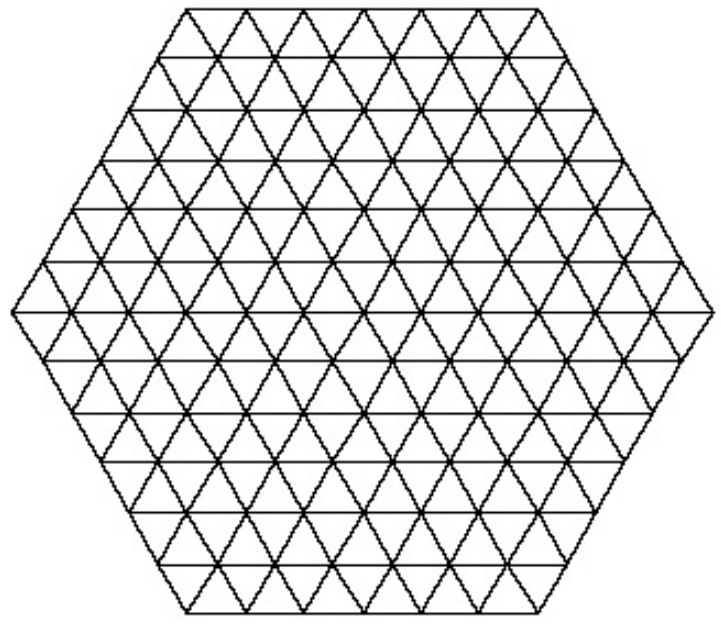

(b) Hexagonal plate

Figure 3-14. Two plate FE layouts

approximation of the data is also shown on these plots and is

$$
\Delta \omega[\%]=175\left(F_{\lambda}^{-2.2808}\right)
$$

It can be seen in the free case, Fig. 3-16 (a), that the lines have a slightly different slope. This is most likely due, as in the beam case, to evanescent behavior in the plate with free boundary conditions. The evanescence lengthens the effective spatial wavelength of the mode, decreases the effective spatial frequency and increases the effective fidelity. Therefore a smaller amount of fidelity is required to achieve a given level of modal frequency error. The effect of evanescence is more prominent in the lower modeshapes, because higher modeshapes have a greater area in the center of the plate over which the evanescence has a much lower effect on the modeshape.

Also in Fig. 3-16 (a), two lines fall significantly below Eqn. 3.25 and are encircled. This is due to the FE layout shown in Fig. 3-14 (a). The modeshapes that these lines represent happen to have relatively high strain along the triangular elements and relatively low strain in the quadrilateral elements. Since the triangular elements are smaller than the quadrilateral elements, the effective stiffness along the triangular elements is smaller than along the quadrilateral elements. In general, as a finite element mesh becomes finer, 
the stiffness and the modal frequencies of the system decrease [11]. The two modes indicated here have an artificially lowered stiffness due to the FE layout, therefore their modal frequencies are closer to that of the true modes.

The MAC is calculated for both boundary conditions and is shown in Figs. 3-15 (b) and Fig. 3-16 (b). The MAC is defined in Eqn. 3.13, and it measures how closely the modeshape of each model, $\phi_{X}$ is to the true modeshape, $\phi$. The MAC can vary between 0 and 1 , with 1 indicating perfect correlation to the true modeshape. The true modeshape in this case is taken to be the modeshape of a highly meshed model. 3 In these plate cases, the models switch from low to high MAC values at fidelities greater than one. In the pinned case, this switch happens at a fidelity level of about 3, in the free case, 4 . These lines are shown in both plots in Figs. 3-15 and Fig. 3-16. Since the FE layout of the circular plate is irregular, fidelity greater than the Nyquist requirement of 1.0 is needed to represent each modeshape.

For the circular plate, the fidelity relationship shown in Eqn. 3.25 is valid for fidelity levels between 1.0 and 72.5 , corresponding to the Nyquist minimum requirement and the $0.01 \%$ precision level, respectively. It is recommended, that for accurate modeshape representation on the circular plate, a fidelity level greater than 4.0 is used.

In the hexagonal plate case, the number of triangular element rings is varied from 2 to 49 . The fidelity is calculated as in the circular plate case, using Eqn. 3.24. The modal frequency error is calculated by taking the "truth" model as a highly meshed plate. ${ }^{4}$ The results can be seen in Figs. 3-17 and 3-18. Figs. 3-17 (a) shows the fidelity relationship for the pinned case and Fig. 3-18 (a) shows the free-free boundary condition. There is a data point for each of the 48 beam models and for each of the ten modes, totaling 480 points on each plot. In addition, boundary lines are drawn at a fidelity level of 1.0 and a modal frequency error of $0.01 \%$, defining the minimum fidelity required and the precision of the calculation, respectively. While the data points do not fall roughly along a straight line, they do seem to have a upper boundary on the maximum fidelity required for a given modal frequency error. In both plots the relation in Eqn. 3.25 is also plotted. This relation provides a mostly conservative boundary on this data. An exception is in Fig. 3-17 a) where the first mode violates Eqn. 3.25 for high frequencies. It is not clear what causes this "pop-up" region.

In addition, the MAC values for each pinned and free hexagonal plates are shown in Figs. 3-17 (b)

\footnotetext{
${ }^{3}$ Each plate in the truth model contains 50 element rings.

${ }^{4}$ The truth model for hex plate contains 50 element rings
} 
and 3-18 (b). In the same way as the circular plates, fidelity levels greater than 1.0 are needed to faithfully represent the modeshapes. In the pinned case, this value is about 1.5 and in the free case, it is 2.2.

\subsubsection{Plate fidelity summary}

For circular plates, the Figs. 3-11 and 3-12 show the relationships between fidelity and modal frequency error for the first ten resonant modes. The fidelity relation shown in Eqn. 3.25 is called the Generalized Plate Relation and is used for plates of any shape. It is valid for fidelity levels greater than 1.0 and less than 72.5 (this corresponds to a modal frequency error of $0.01 \%$ ). A recommended minimum fidelity for plates, though, is 4.0 to ensure that the displacements associated with the modeshapes are represented accurately. It is important to accurately represent displacements, since these displacements are in many cases used to calculate the output figures of merit. Figure 3-19 compares the fidelity relationships for the circular and generalized plate. Three lines show circular azimuthal, circular radial and generalized plate fidelity relationships. It is important to note that these lines are plotted against the azimuthal fidelity, radial fidelity and generalized fidelity respectively. Therefore, the Fidelity axis is not only generalized fidelity. Data points representing the "knee" points of the combinations modes 4, 7, and 10, shown in Fig. 3-12, are also plotted in Fig. 3-19. These points are plotted against the azimuthal and radial fidelities for each mode. It can be seen that the combination mode knee points require more fidelity in the azimuthal and radial directions to achieve the same level of modal frequency error compared to the generalized case. It can be seen that the azimuthal and radial fidelity relationships for the circular plate approximately agree with the generalized plate, but that they violate the Generalized Plate Relation. This is primarily due to the different finite element (FE) layouts. In the generalized plate case, the finite elements are approximately the same size. In the circular plate case, the finite element layout is such that some elements are much larger than others.

In summary, the fidelity relationships shown in this section are used to assess fidelity of existing plate models and to create new known fidelity plate models. These methods are described in Section 3.6 and Chapter 4, respectively. 


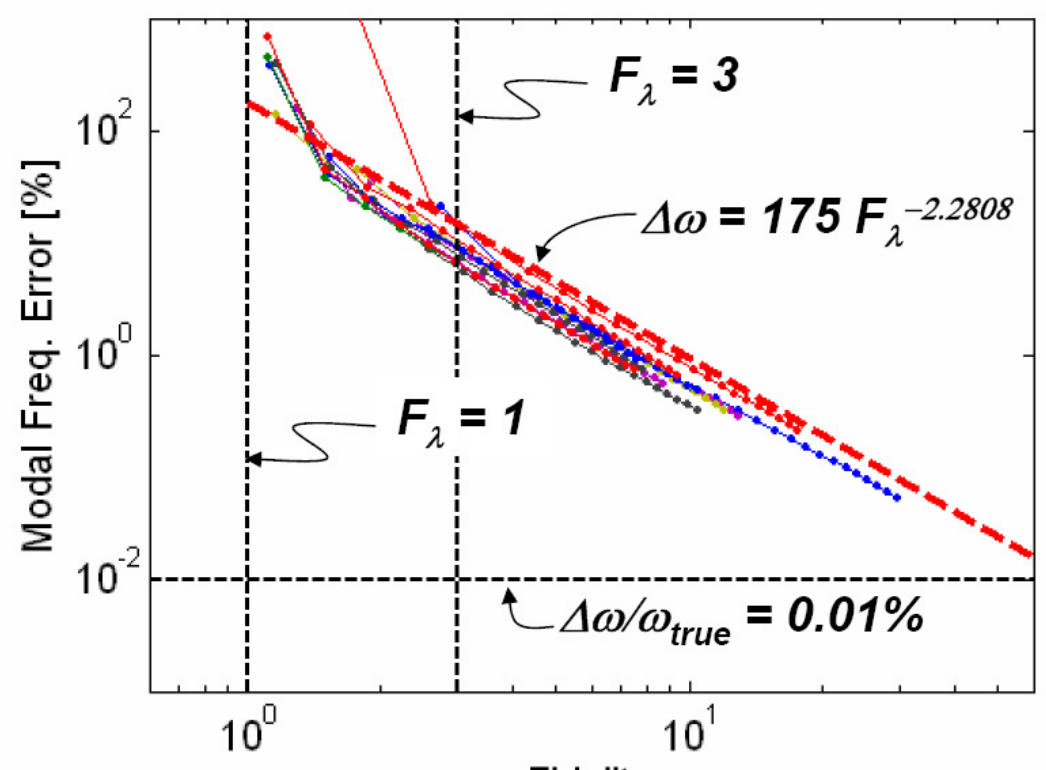

Fidelity

(a) Fidelity relationship for first 10 modes

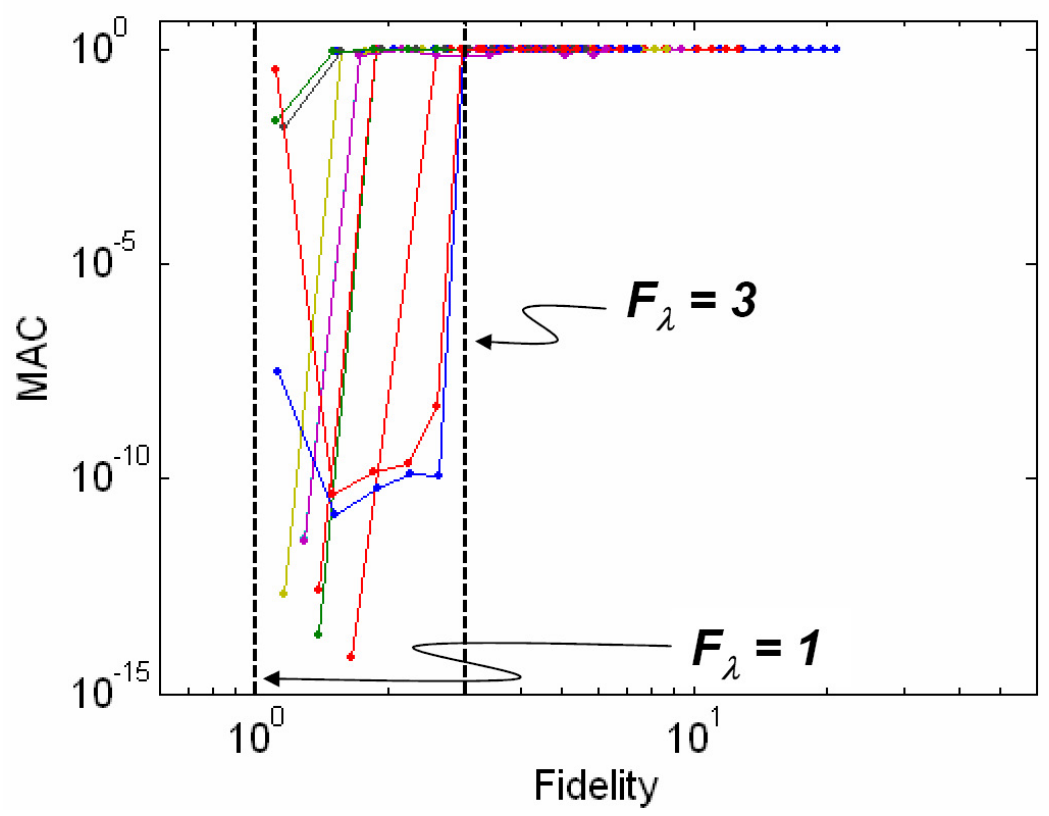

(b) MAC values for first 10 modes

Figure 3-15. Pinned circular plate 


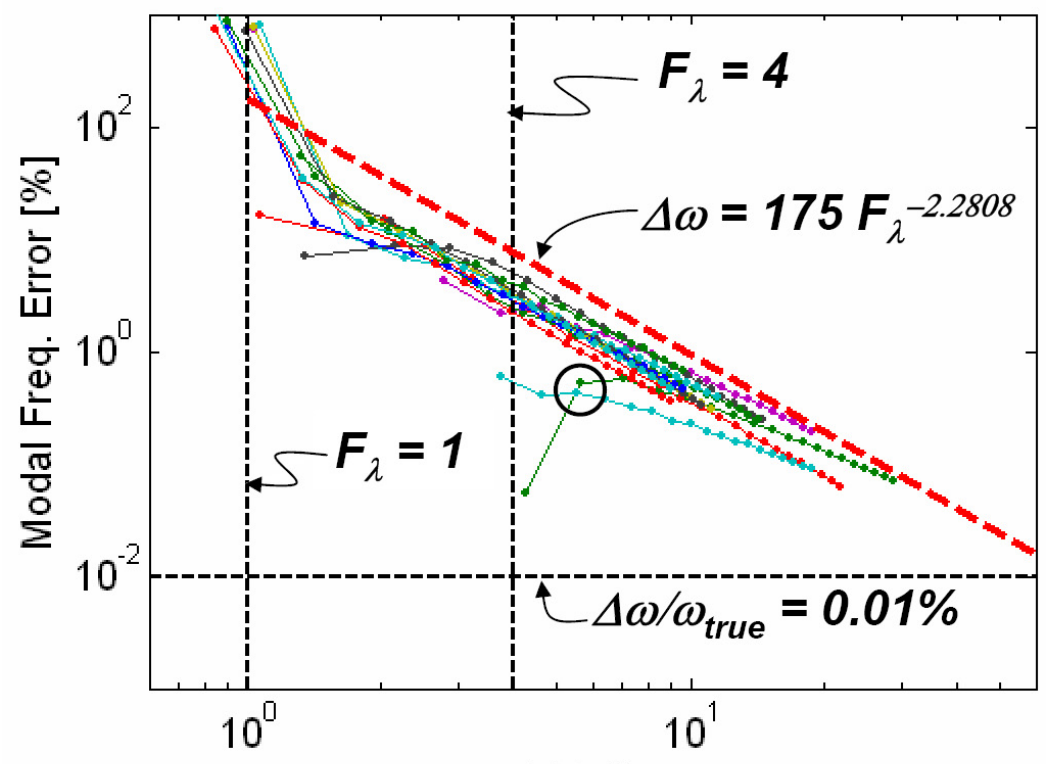

Fidelity

(a) Fidelity relationship for first 10 modes

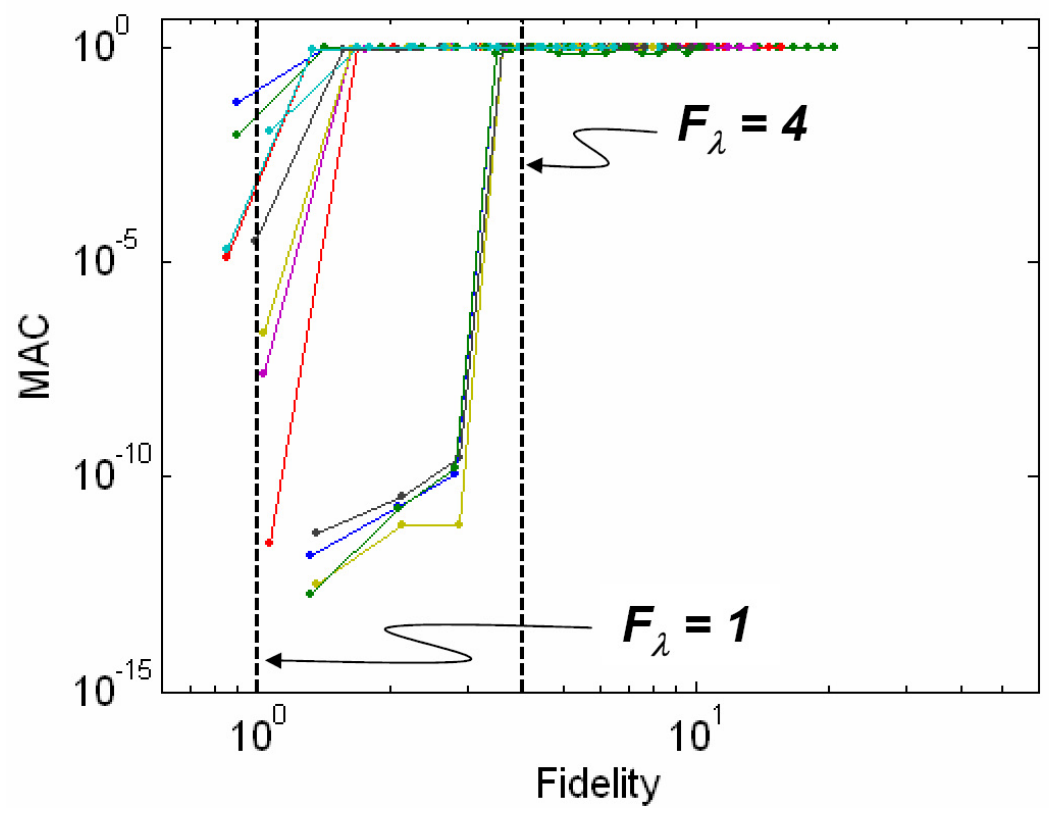

(b) MAC values for first 10 modes

Figure 3-16. Free circular plate 


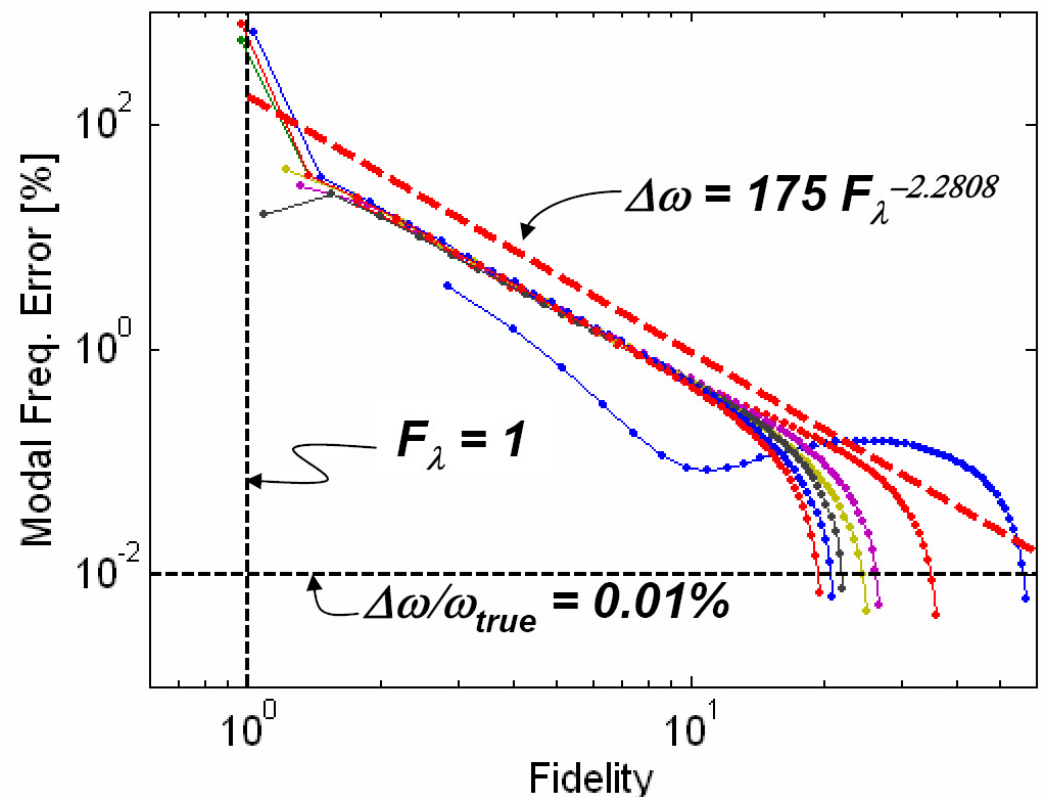

(a) Fidelity relationship for first 10 modes

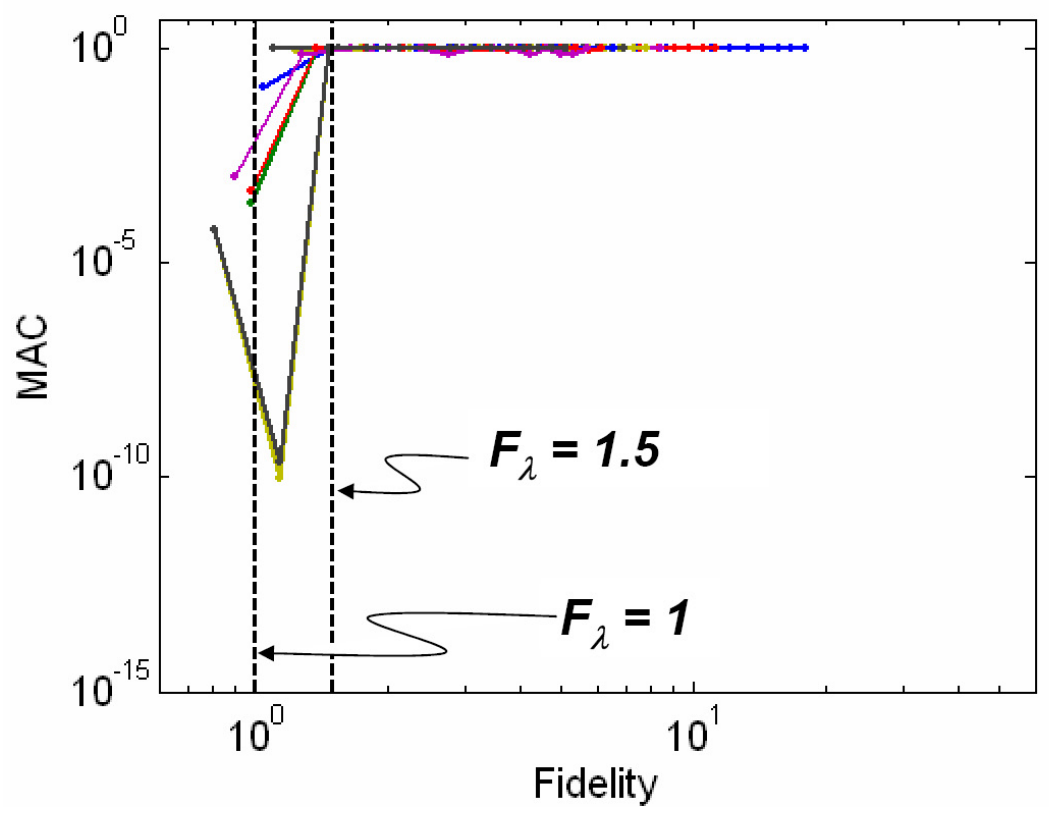

(b) MAC values for first 10 modes

Figure 3-17. Pinned hex plate 


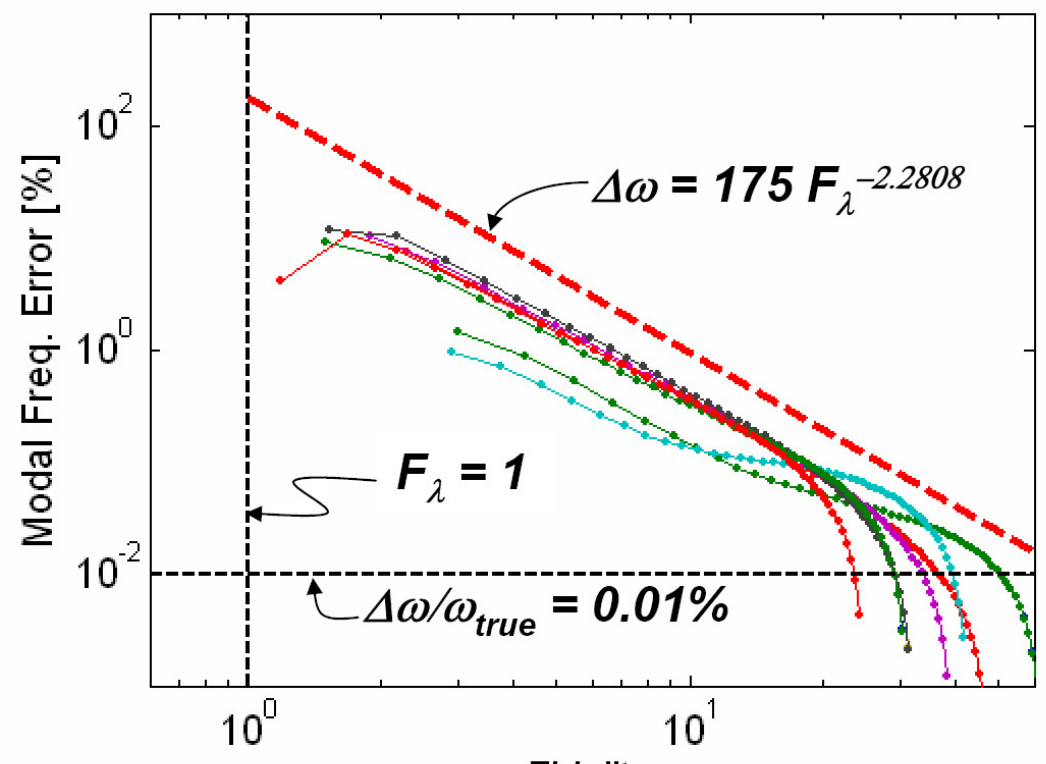

Fidelity

(a) Fidelity relationship for first 10 modes

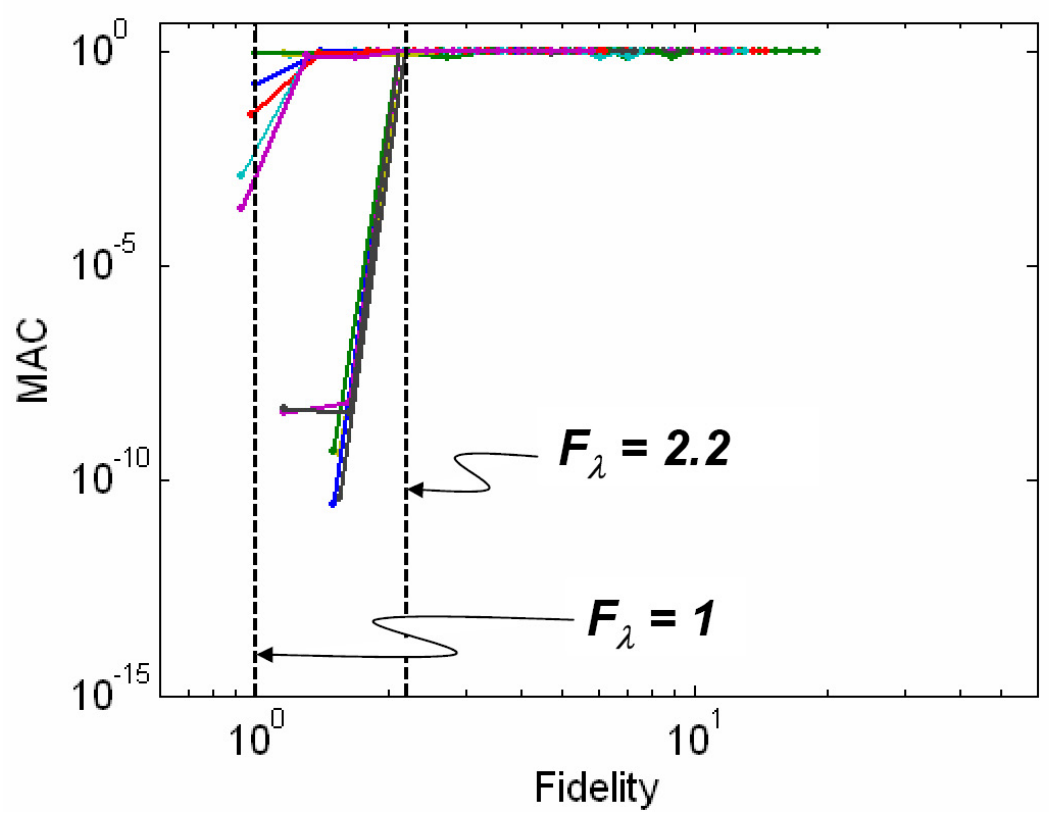

(b) MAC values for first 10 modes

Figure 3-18. Free hex plate 


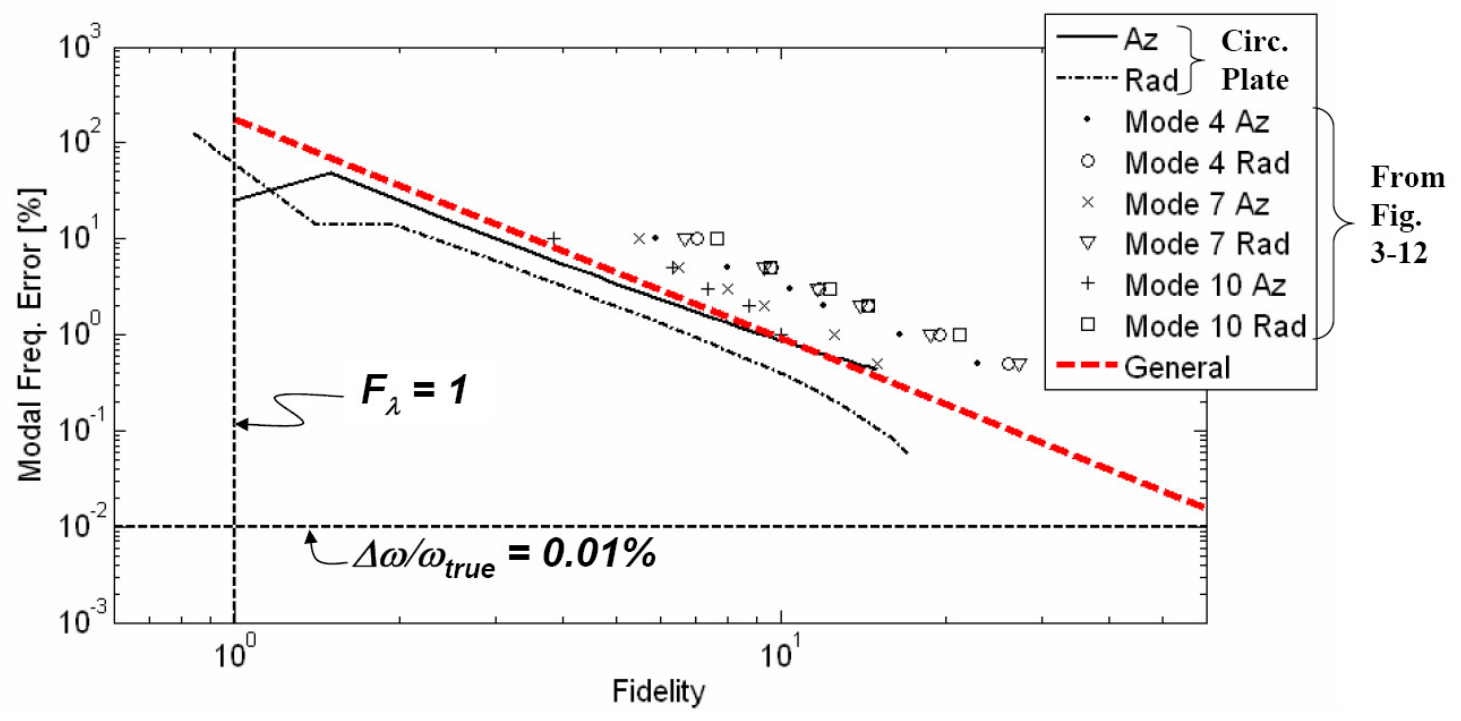

Figure 3-19. Comparison of circular and generalized plate relations

\subsection{Output Error Estimates}

In addition to the modal frequency error estimates, sensitivity techniques discussed in Section 2.2 .3 can be used to calculate an a posteriori estimate of the error bound on the output figure of merit (OFM) of the integrated model. For this analysis, the OFM must be a linear combination of the outputs of the system state space integrated model.

Once the system integrated model matrices are assembled, a sensitivity analysis is performed on the system. The result is an estimate of the change in the OFM with respect to changes in the modal frequencies,

$$
\frac{\partial \sigma_{z_{i}}}{\partial \omega_{k}} \approx \frac{\Delta \sigma_{z_{i}}}{\Delta \omega_{k}}
$$

where

$$
\begin{aligned}
i & =1 \ldots n \\
k & =1 \ldots m
\end{aligned}
$$

Here, $n$ is the number of OFM and $m$ is the number of system modal frequencies included in the estimate. The percent change of the OFM with respect to the percent change in the modal frequencies can be estimated 
as,

$$
\frac{\% \text { change in } \sigma_{z_{i}}}{\% \text { change in } \omega_{k}} \approx \frac{\delta \sigma_{z_{i}} / \sigma_{z_{i}, \text { nom }}}{\delta \omega_{k} / \omega_{k, n o m}}=\frac{\delta \sigma_{z_{i}}}{\delta \omega_{k}} \frac{\omega_{k, n o m}}{\sigma_{z_{i}, \text { nom }}}
$$

where $\sigma_{z_{i}, \text { nom }}$ and $\omega_{k, \text { nom }}$ are the nominal structural modal frequencies and the OFMs, respectively.

Finally the error bound of the OFM can be estimated by multiplying the quantity in Eqn. 3.28 by the modal frequency error specified, MFE, and then summing over the modal frequencies,

$$
\frac{\Delta \sigma_{z_{i}}}{\sigma_{z_{i}, \text { nom }}}=\sum_{k=1}^{m}\left|\frac{\% \text { changein } \sigma_{z_{i}}}{\% \text { changein } \omega_{k}}\right|(\mathrm{MFE})
$$

The modal frequency error, MFE, is estimated using the fidelity relations shown in the previous sections. In the Nyquist fidelity method for assessment, described next in Section 3.6. MFE is taken to be the maximum modal frequency error across the components. In the Nyquist fidelity method for synthesis, described in Chapter 4, MFE is the modal error specified.

Equation 3.29 is not an absolute measurement of OFM error, since the quantity in Eqn. 3.28 represents a change in the OFM of the model analyzed, not a change in the OFM of the true system. But, if the model is assumed to be close to the true system, this method is a useful tool in estimating the OFM error in the absence of a truth model because in some cases it incurs a lower computational cost than running a high fidelity model. Also, this is a error bound estimate because it assumes that the maximum modal frequency error of the full system coupled modes is equal to or less than the modal frequency error present in the component models. This is a key assumption that allows the fidelity metric, defined on system components, to be applied to the full system.

\subsection{Fidelity Assessment Method}

The Nyquist method can be used to generate new models or to assess existing models. The Nyquist fidelity assessment procedure of structural component models consists of five steps enumerated below.

1. Identify model properties, including model geometry, upper disturbance frequencies and OFM.

2. Identify important component models using network diagrams. 
3. Calculate fidelity metric based on mesh density and upper disturbance frequency for each component. System fidelity is the minimum fidelity present in the components.

4. Estimate modal frequency error based on relationships between fidelity and modal accuracy. Modal frequency error estimate is the maximum modal frequency error estimate across components.

5. Estimate OFM error based on system modal frequency error.

First, the model properties are identified. This includes the model configuration, OFM and the disturbances imparted to the model. It is important to identify the upper disturbance frequency because that frequency is used in the fidelity metric.

Next, components that lie along the disturbance path from the disturbance source to the location of the OFM measurement are identified. These are called important components because it is likely that they contribute the most to the OFM. The important components are identified using network diagrams as in Fig. 3-21. This is further discussed in the next section.

Then the actual fidelities are calculated for each component based on the upper disturbance frequency and its mesh size. After the fidelities are calculated, the modal frequency error in each component is estimated using the previously derived relationships. The fidelity of the system is taken to be the minimum fidelity across the components. In a similar way, the system modal frequency error is taken to be the maximum modal frequency error over the components.

Finally the OFM error is calculated using sensitivity methods. This step is useful in the assessment of any model, since it provides a indication of the utility of the model. This method is demonstrated in detail using the MOST system as an example in the next section.

\subsection{Fidelity Assessment of MOST Nominal Configuration}

The method of fidelity assessment is applied to the MOST system, which was introduced in Section 2.3 . The MOST configuration is shown in Fig. 3-20. This model was created a priori without using the Nyquist fidelity method. 


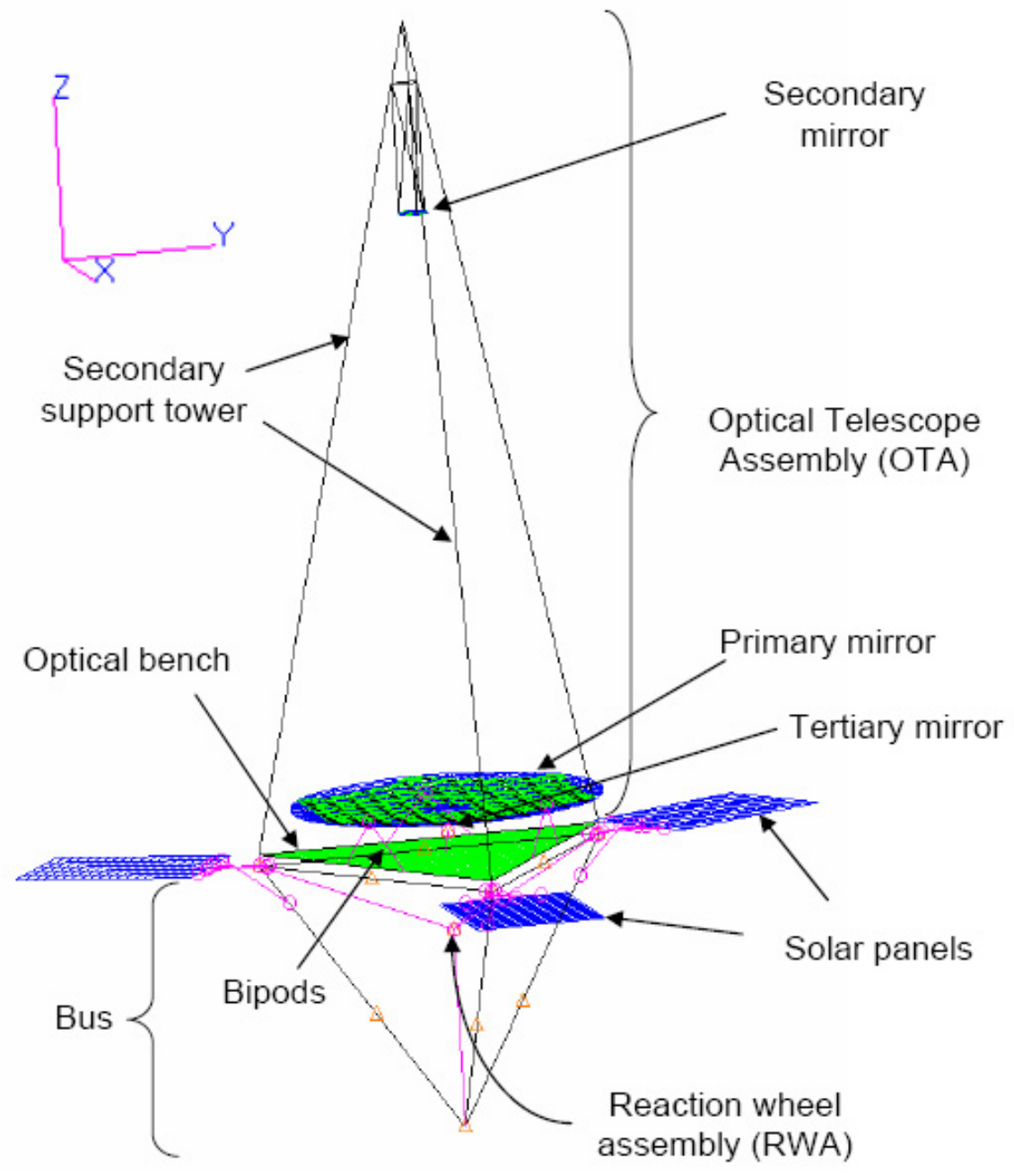

Figure 3-20. MOST nominal configuration 


\subsubsection{Initialize model properties}

The first step in fidelity assessment is to define the model to be analyzed. Also defined in this step are the MOST nominal parameters, shown in Table 2.2 and the geometry, connectivity, and the material properties. The nominal configuration of MOST can be seen in Fig. 2-5. The nominal parameter values can be seen in Table 2.2, the material and geometric properties are listed in Table 3.5 and 3.6 . The MOST finite element model is made up of beam, plate, rigid body, and concentrated mass elements. The secondary support tower (SST) contains three main support legs and nine smaller beams that support the secondary mirror. These are modeled using beam elements. In Table 3.5, SST beams are categorized by length. The three main support beams are each $7.81 \mathrm{~m}$ long. The smaller secondary mirror support beams are $1.21 \mathrm{~m}, 1.18 \mathrm{~m}$ and $0.25 \mathrm{~m}$ long. There are three beams of each length. Each beam also has a hollow cross section, with the geometric properties shown in Table 3.5 .

The primary mirror, secondary mirror, optical bench and solar panels are made up of plate elements. The primary and secondary mirrors are circular plates, the optical bench is a triangular plate and the solar panels are rectangular plates. The material and geometric properties of all MOST plates are listed in Table 3.6 showing flexural rigidity, $D$, which is calculated using Eqn. 3.22 .

The remaining sections of the model are the bus and the tertiary mirror. The bus is made up of rigid body and concentrated mass elements. The tertiary mirror is modeled as a concentrated mass.

The disturbance spectra are shown in Fig. 2-9. The upper frequency is shown to be $100 \mathrm{~Hz}$. This is important to define because this frequency is involved in the fidelity metric calculation. Next, the output figures of merit (OFM) are defined. In this case, the OFM are LOS jitter and WFE. These are optical performance metrics that measure the degradation of the science light. They are calculated based on the displacements of the optical surfaces (mirrors), see Section 2.3.3.

\subsubsection{Critical component identification}

The next step is to identify critical or important components. The important component models are identified through the subsystem decomposition, shown in Fig. 3-21. MOST consists of eight main structures depicted in the boxes. The main disturbances, shown as a solid arrow, are the forces due to the imbalance in the reaction wheels. They originate from within the bus. The science light, shown as the dashed arrow, enters 
Table 3.5. Material and geometric properties for MOST beams

\begin{tabular}{|c|c|c|}
\hline Beam lengths [m] & 7.81 & 1.18 \\
\hline$\rho\left[\mathrm{kg} / \mathrm{m}^{3}\right]$ & 1550 & 1550 \\
\hline$E[P a]$ & $5.17 \mathrm{E}+10$ & $5.17 \mathrm{E}+10$ \\
\hline$I\left[m^{4}\right]$ & $4.31 \mathrm{E}-07$ & $1.47 \mathrm{E}-09$ \\
\hline Major axis diameter $[\mathrm{m}]$ & 0.06 & 0.03 \\
\hline Minor axis diameter $[\mathrm{cm}]$ & 0.74 & 0.01 \\
\hline Wall thickness $[m]$ & $1.22 \mathrm{E}-03$ & 0.01 \\
\hline Cross sectional Area $\left[\mathrm{m}^{2}\right]$ & $1.22 \mathrm{E}-03$ & $2.36 \mathrm{E}-04$ \\
\hline
\end{tabular}

Table 3.6. Material and geometric properties for MOST plates

\begin{tabular}{rcccc}
\hline \hline & Primary & Secondary & Optical & Solar \\
& Mirror & Mirror & Bench & Panel \\
\hline Young's modulus (E) $[\mathrm{Pa}]$ & $3.75 \mathrm{e} 11$ & $3.75 \mathrm{e} 11$ & $8.24 \mathrm{e} 10$ & $7.194 \mathrm{e} 10$ \\
Poisson's ratio $\nu$ & 0.17 & 0.17 & 0.45 & 0.29 \\
$\rho\left[\mathrm{kg} / \mathrm{m}^{3}\right]$ & 3200 & 3200 & 201.77 & 2916 \\
Flexural Rigidity $[\mathrm{Nm}]$ & $3.22 \mathrm{E} 4$ & $3.22 \mathrm{E} 4$ & $8.61 \mathrm{E} 3$ & 43.1583 \\
\hline Area $\left[\mathrm{m}^{2}\right]$ & 7.069 & 0.066 & 2.133 & 2.195 \\
$\mathrm{~h}[\mathrm{~m}]$ & 0.01 & 0.01 & 0.01 & 0.0019 \\
\hline \hline
\end{tabular}

the system and reflects off the primary mirror (PM), then the secondary mirror (SM) then the tertiary mirror (TM) before entering the image plane on the science instruments.

By visual inspection of this network, it can be seen that the mirrors are along the critical path for manipulating the science light. It is the degradation of the science light, measured as LOS jitter and WFE, that affect the OFM. The optical bench and the secondary tower structure (SST) provide support to the optical surfaces (mirrors). It is through the optical bench and SST that the reaction wheel disturbance flows to the optical surfaces. Therefore the optical bench and the SST in addition to the mirrors are the critical components. The solar array system is an appendage whose behavior can influence the system-level coupled modes and therefore the quality of the science light. An appendage such as this, however, will not be treated in the same manner as the critical components since it does not lie in the disturbance path. The fidelity calculation of the appendages will be based on the frequency of its fundamental mode. Further research into the effects of appendage modes on coupled modes in this context would be useful.

The bus is modeled as a rigid body. Even though the disturbance originates there, since it is a rigid body, no modal content is present, therefore it is not considered an important component from a structural 

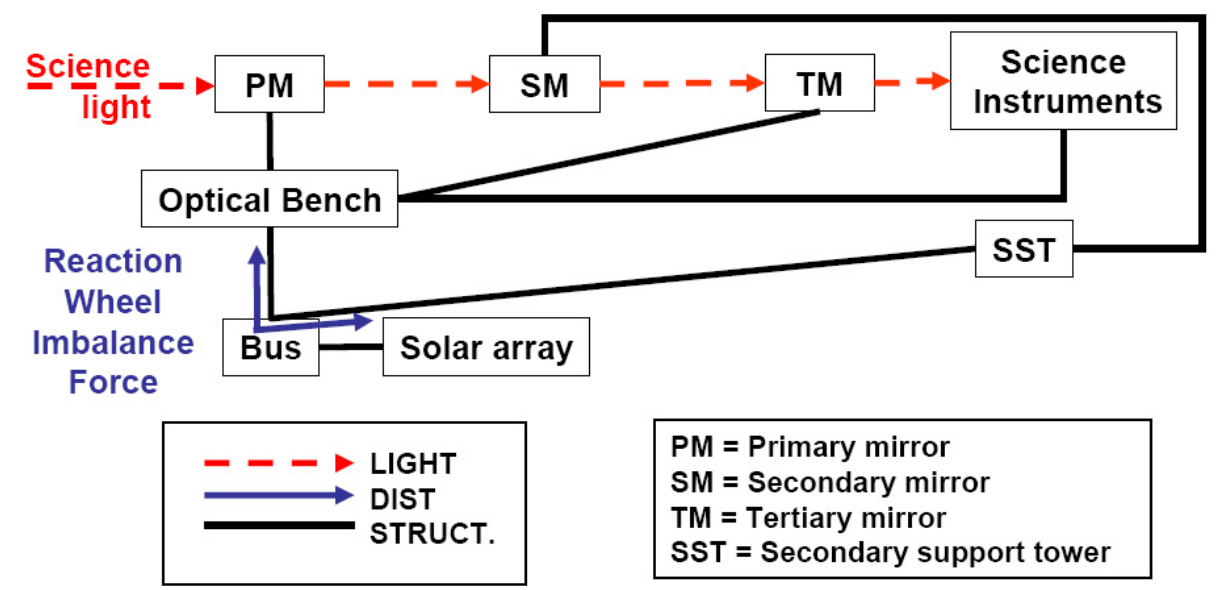

Figure 3-21. Network diagram for MOST system

dynamics standpoint. However, the bus mass and inertia are important.

The primary and secondary mirrors, the optical bench and the solar panels are all modeled as plates. The SST is composed of beams of 4 different sizes and geometries as shown in Fig. 3-22, It is appropriate to model the tertiary mirror as a concentrated mass due to its relatively small size (discussed below). These plates and the SST beams will be examined in this section.

\subsubsection{Fidelity metrics and modal frequency error estimation}

The second step is to calculate the fidelity levels based on the $100 \mathrm{~Hz}$ upper disturbance frequency and the nominal mesh densities. The generalized beam and plate fidelity metrics, Eqns. 3.12 and 3.25 , are used for all the components identified. In addition, azimuthal and radial fidelities using the circular plate fidelity metrics are assessed for the primary and secondary mirrors. A discussion on calculating the fidelities is given below.

\section{Generalized beam fidelities}

The generalized beam relation in Eqn. 3.12, repeated here for convenience,

$$
\operatorname{MFE}[\%]=\Delta \omega[\%]=10^{0.7579}\left(\mathrm{~F}_{\lambda}\right)^{-3.907}
$$




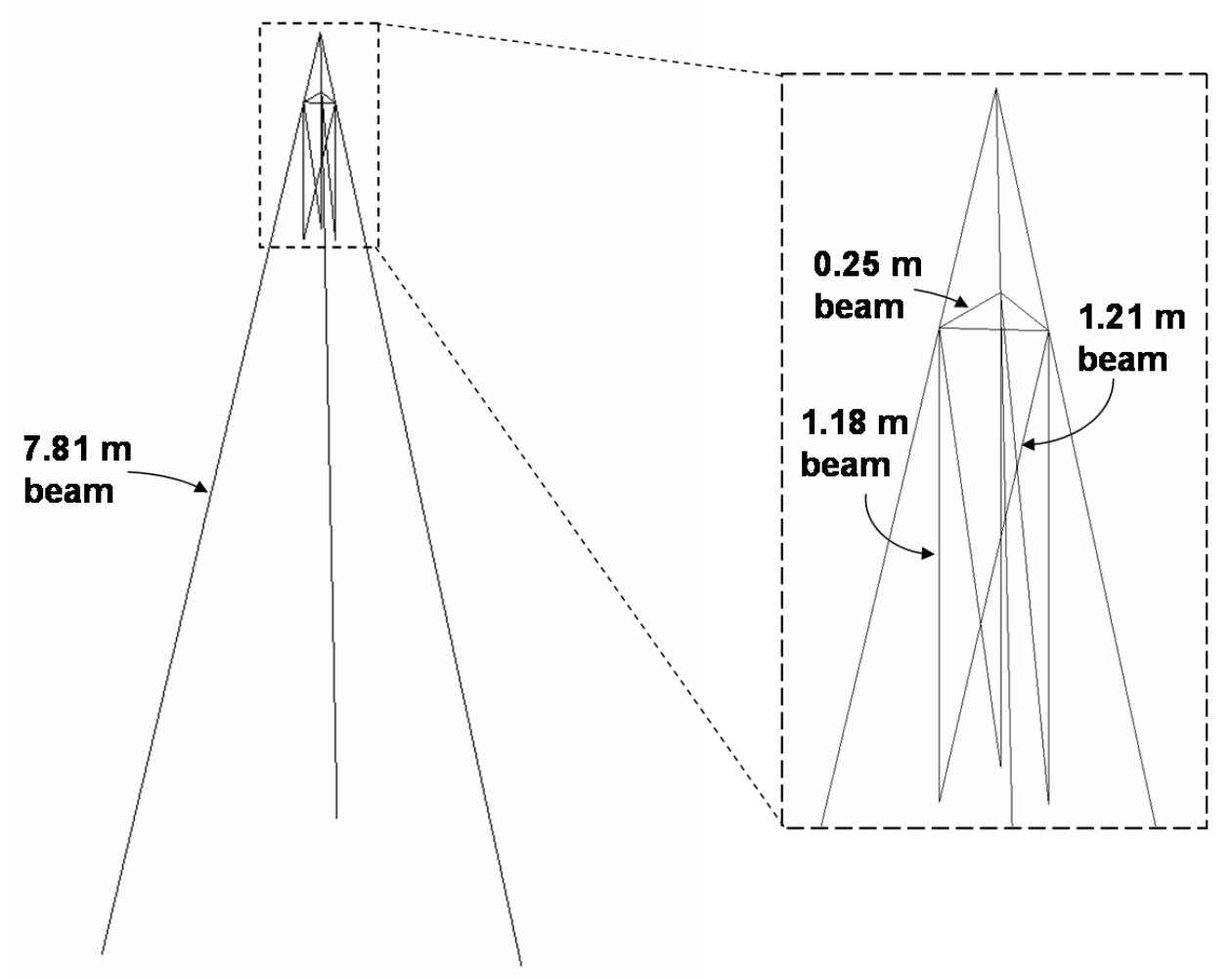

Figure 3-22. Secondary support tower of MOST

calculates the fidelity of a beam with a given mesh. The SST is shown in Fig. 3-22 and is composed of three long trip leg beams in a tetrahedral tower configuration, three short beams in the mirror plane that connect the long beams at the top, and six short beams which connect the tower to the secondary mirror. The long leg beams are meshed such that there is a node every $0.4 \mathrm{~m}$. The three shorter beams are modeled as one element each. Therefore they contain 2 nodes per beam. The sampling frequencies, the numerator of Eqn. 3.2, are simply the number of nodes in the beam mesh divided by its length. The denominator of Eqn. 3.2 is twice the spatial frequency. The spatial frequency is obtained using the wave propagation equations given in Section 3.3.1, where $\omega$ is the upper disturbance frequency of $100 \mathrm{~Hz}$. Since these beams have the same material and cross sectional properties, their spatial frequencies are the same. Once the fidelity is obtained, the modal frequency error is estimated using the generalized beam relation given in Eqn. 3.12. These results are shown in Table 3.7. The $1.21 \mathrm{~m}$ and $1.18 \mathrm{~m}$ beams both have a fidelity level of less than 1.0. This indicates that these beam models contain insufficient fidelity to represent modes up 
to $100 \mathrm{~Hz}$. Furthermore, some or all of the modes that are calculated using these beam models are aliased. Therefore, these beams cannot reliably represent frequencies below $100 \mathrm{~Hz}$. Their corresponding modal frequency errors are not shown here because the generalized beam relation is not valid for fidelity levels less than 1.0, however the modal frequency errors are likely to exceed $10 \%$. Since these beams transmit the disturbance forces to the secondary mirror (see Fig. 3-21), the response of the SM to the disturbances cannot be accurately calculated when using frequency domain analyses. This is because the frequency domain analyses use modal frequency and modeshape estimates to estimate performance. If these modes or modeshapes are aliased, then the performance estimate is inaccurate. The longest and the shortest beams, $7.81 \mathrm{~m}$ and $0.25 \mathrm{~m}$, both exceed the fidelity requirement of 1.0. Their corresponding modal frequency errors are both less than $0.06 \%$. This indicates that the FE mesh sizes are sufficiently small such that a modal frequency error of the system due to the undersampling of the beam models is at most $0.06 \%$.

Table 3.7. Fidelity assessment results for MOST beams

\begin{tabular}{r|ccccc}
\hline \hline & \multicolumn{5}{|c}{ Sampling freq } \\
Beam Lengths [m] & \# nodes & n [nodes/m] & $\varpi$ for 100Hz & $F_{\lambda}$ & MFE [\%] \\
\hline 7.81 & 20 & 2.56 & 0.3831 & 3.2632 & 0.0564 \\
1.21 & 2 & 1.66 & 1.0499 & 0.7886 & $>10 \%$ \\
1.18 & 2 & 1.69 & 1.0499 & 0.8062 & $>10 \%$ \\
0.25 & 2 & 7.96 & 1.0499 & 3.7895 & 0.0314 \\
\hline \hline
\end{tabular}

\section{Circular Plate Fidelities (primary and secondary mirrors)}

Next, the fidelities of the primary and secondary mirrors are assessed using the circular plate fidelity metrics. The radial and azimuthal plate fidelities shown in Eqn. 3.18 are applied to the primary, secondary and tertiary mirrors. The layout of the primary and secondary mirrors can be seen in Fig. 3-14(a). The tertiary mirror is modeled as a concentrated mass, but its diameter and material properties are used to evaluate its fidelity.

First, the numerators of the metrics shown in Eqn. 3.18, $\left(n_{r}\right.$ and $\left.n_{\theta}\right)$ are obtained using the nominal mesh. The primary mirror is meshed with 10 rings and is 3 meters in diameter in the nominal case. The secondary mirror has 3 element rings and is 0.29 meters in diameter. These are the sampling frequencies. The radial sampling frequency is the number of nodes across the diameter divided by the diameter. In the 
case of element rings as in Fig. 3-14(a), the number of nodes, $n_{\text {nodes }}$, across the diameter is

$$
n_{\text {nodes }}=2 n_{\text {rings }}+1
$$

where $n_{\text {rings }}$ is the number of rings. And the radial sampling frequency is

$$
n_{r}=\frac{2 n_{\text {rings }}+1}{D_{0}}[\text { cycles } / m]
$$

where $D_{0}$ is the diameter. Because of the mesh layout in Fig. 3-14 (a), there is no constant azimuthal frequency. The azimuthal sampling frequency is approximated as the azimuthal spatial frequency of the element ring located at one-half radius. The azimuthal spatial frequency of this "center" ring is the number of nodes in the center ring divided by $2 \pi$ radians,

$$
n_{\theta}=\frac{6+6\left(n_{\text {rings }} / 2\right)}{2 \pi}
$$

The numerator of Eqn. 3.31 is found by inspection of the FE layout in Fig. 3-14(a). In the case of an odd $n_{\text {rings }}$, the quantity $\left(n_{\text {rings }} / 2\right)$ is rounded down to the next lower integer, effectively selecting the smaller of the two center rings. The sampling frequencies can be seen in Table 3.8

Table 3.8. Primary and secondary mirror sampling frequencies

\begin{tabular}{r|cc|cc}
\hline \hline & & & \multicolumn{2}{|c}{ Sampling Frequency } \\
& $D_{0}[\mathrm{~m}]$ & \# element rings & $n_{r}$ & $n_{\theta}$ \\
\hline PM [nodes $/ \mathrm{m}]$ & 3 & 10 & 7.00 & 5.73 \\
SM [nodes $/ \mathrm{rad}]$ & 0.29 & 3 & 24.12 & 1.91 \\
\hline \hline
\end{tabular}

Next, the denominators of Eqn. 3.18, the Nyquist sampling requirements, are calculated. In the circular plate fidelity case, this is obtained by first estimating the modal frequencies of the plate, then calculating the spatial frequency of the mode just beyond the disturbance frequency bound. In the generalized plate fidelity case, in the next section, the Nyquist requirement is obtained differently. Table 3.9 shows the first 10 modal frequencies of the primary and secondary mirrors. This is from the Leissa [48] calculation with free-free boundary conditions. This is only an estimate because these plates have different boundary conditions than those used by Leissa. Also the Leissa numbers are for a Poisson ratio of 0.33 , whereas these plates have a 
Poisson ratio of 0.17 .

Table 3.9. Estimated modal frequencies of the primary secondary and tertiary mirrors

\begin{tabular}{r|ccccc}
\hline \hline Mode No. & 1 & 2 & 3 & 4 & 5 \\
\hline PM [Hz] & 11.8 & 20.4 & 27.4 & 46.0 & 48.5 \\
SM [Hz] & 1259 & 2177.2 & 2931.2 & 4918.1 & 5177 \\
TM [Hz] & 16570 & 28655 & 38578 & 64729 & 68135 \\
& & & & & \\
\hline Mode No. & 6 & 7 & 8 & 9 & 10 \\
\hline PM [Hz] & 74.2 & 79.1 & 86.5 & 103.6 & 118.7 \\
SM [Hz] & 7933.2 & 8448.5 & 9239.4 & 11073 & 12681 \\
TM [Hz] & $1.0 \mathrm{E} 5$ & $1.1 \mathrm{E} 5$ & $1.2 \mathrm{E} 5$ & $1.5 \mathrm{E} 5$ & $1.7 \mathrm{E} 5$ \\
\hline \hline
\end{tabular}

It can be seen that the 9th mode of the primary mirror is just past the $100 \mathrm{~Hz}$ disturbance frequency boundary. The cases of the secondary and tertiary mirrors are different. Their first frequencies are significantly above the $100 \mathrm{~Hz}$ boundary. Therefore, the SM and TM modes do not contribute to the system dynamics and the fineness of their meshes do not incur modal frequency error penalties.

Next, the corresponding radial and azimuthal spatial frequencies of Mode 9 of the PM are calculated, again using Leissa [48]. Since mode 9 is a purely azimuthal mode, it only has azimuthal frequency, which is

$$
\varpi_{\theta, 9}=0.955[\text { cycles/rad }]
$$

We also look into the spatial frequencies of the next lowest modes that are combination and radial modes: modes 7 and 8 , respectively. Their spatial frequencies are

$$
\begin{aligned}
& \varpi_{r, 7}=0.2028[\text { cycles } / \mathrm{m}] \\
& \varpi_{\theta, 7}=0.3183[\text { cycles } / \text { rad }] \\
& \varpi_{r, 8}=0.5945[\text { cycles } / \mathrm{m}]
\end{aligned}
$$

Note that since mode 8 is a purely radial mode, it has no associated azimuthal spatial frequency. From these numbers we can calculate the azimuthal and radial fidelities based on the mesh density of the PM and the spatial frequencies of modes 7, 8, and 9. These are shown in Table 3.10. Using the plots in Figs. 3-11 and 3-12, the modal frequency estimates can be obtained; these are also shown in Table 3.10 .

The fidelity levels of the primary mirror model in each direction are taken as the minimum in each 
Table 3.10. Primary mirror fidelity levels

\begin{tabular}{r|ccc}
\hline \hline Mode No. & $F_{r}$ & $F_{\theta}$ & MFE [\%] \\
\hline 7 & 17.262 & 9.0 & 0.15 \\
8 & 5.887 & - & 1.75 \\
9 & - & 3.0 & 10 \\
\hline \hline
\end{tabular}

direction over the modes examined, which are

$$
\begin{aligned}
& F_{\theta}=3.0 \\
& F_{r}=5.887 .
\end{aligned}
$$

The recommended minimum fidelity level discussed in Section 3.4 was 4.0. The primary mirror, therefore does not meet minimum recommended fidelity in the azimuthal direction.

\section{Generalized Plate Fidelities}

An alternative to the fidelity calculation above is the generalized plate fidelity (Eqn. 3.24) which makes use of the wave propagation equation for plates. First, the numerator of Eqn. 3.24 is calculated, which is the sampling frequencies of each mesh. Since it was shown that the modal frequency estimates of the secondary and tertiary mirrors significantly exceed the disturbance upper frequency bound, their meshes are not analyzed here. The meshes and sampling frequencies of the primary mirror, the optical bench and the solar panels are shown in Table 3.11. The optical bench is the triangular bench below the primary mirror. The solar panels are rectangular and there are three of them. The FE meshes for the optical bench and solar panels are shown in Fig. 3-23.

Table 3.11. MOST plate mesh properties

\begin{tabular}{r|ccc}
\hline \hline & \# nodes & Area $\left[m^{2}\right]$ & $\left(\sqrt{\# \text { nodes } / m^{2}}\right)_{\text {FEM }}$ \\
\hline PM & 390 & 7.07 & 7.43 \\
Optical bench & 561 & 2.13 & 16.22 \\
Solar panels & 120 & 2.19 & 7.39 \\
\hline \hline
\end{tabular}

The numerator in Eqn. 3.24 is based on the wave propagation equations which relate time domain frequencies to spatial frequencies for plates, $\varpi$. These equations can be seen in Section 3.4.2. The time domain 


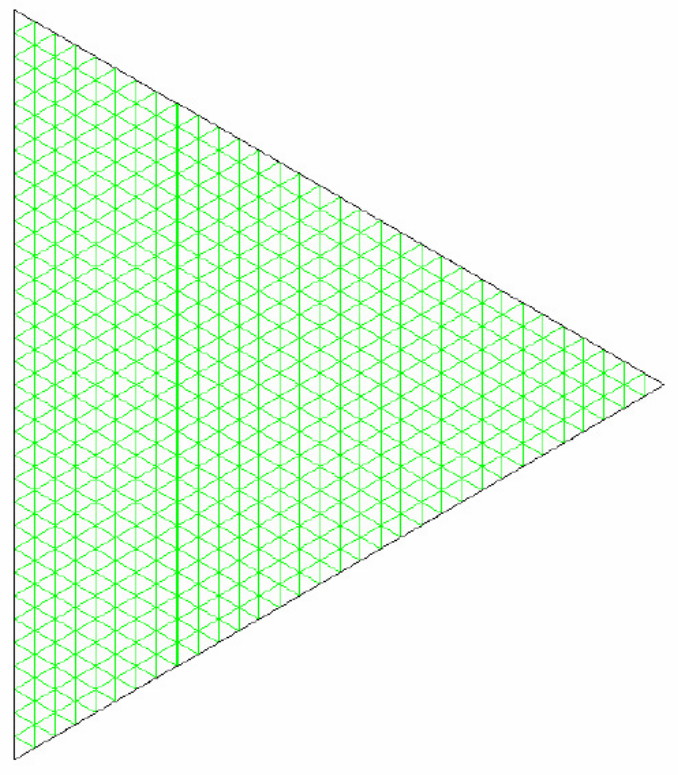

(a) Optical bench

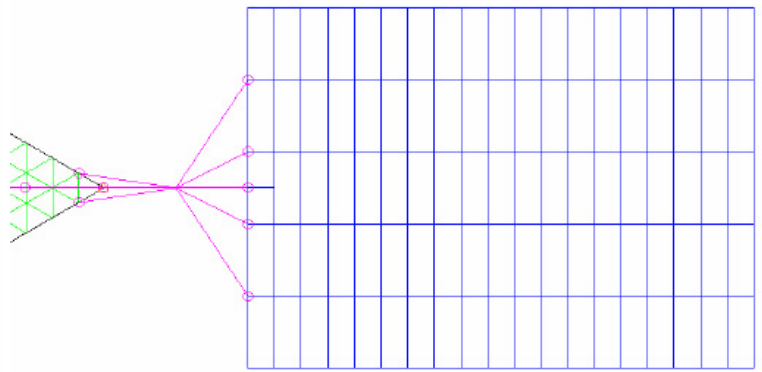

(b) Solar panel

Figure 3-23. FE meshes for plates in the MOST model

frequency used for the primary mirror and the optical bench, $\omega$, is the $100 \mathrm{~Hz}$ upper disturbance. Since the solar panels are appendages, it is important that the fundamental mode is captured, therefore the spatial frequency corresponds to the first modal frequency of the plate. This is estimated using the modal frequency equations for a rectangular plate in Appendix B. Then the fidelities are simply the sampling frequencies divided by twice the spatial frequencies, as in Eqn. 3.24. Finally, the modal frequency errors are calculated using the Generalized Plate Equation (Eqn. 3.25). The plate spatial frequencies, the fidelities, and the modal frequency error estimates are shown in Table 3.12 .

Table 3.12. Fidelity results for MOST plates

\begin{tabular}{r|cccc}
\hline \hline & $\omega[\mathrm{Hz}]$ & $\varpi[$ nodes $/ \mathrm{m}]$ & $F_{\lambda}$ & MFE [\%] \\
\hline PM & 100 & 0.224 & 16.5781 & 0.2894 \\
Optical bench & 100 & 0.1218 & 16.4288 & 0.2955 \\
Solar panels & 0.0417 & 0.0486 & 76.0689 & 0.0090 \\
\hline \hline
\end{tabular}

Note that the generalized plate relation seems to suggest sufficient fidelity for the primary mirror while the circular plate fidelities seem to indicate that it is insufficient $\left(F_{\theta}<4.0\right)$. Since the primary mirror in MOST is meshed in the same layout as the plate used to define the generalized fidelity relation, the 
generalized plate relation and fidelity results, shown in Table 3-23 is preferred.

\section{MOST Fidelity Summary}

The fidelity and modal frequency values of each of the components described in this sections is summarized in Fig. 3-24. The modal frequency errors for the $1.21 \mathrm{~m}$ and $1.18 \mathrm{~m}$ beams are not shown because their models do not meet the minimum fidelity requirement for beams of 1.0. The modal frequency error for the solar panels is rounded up to $0.01 \%$ because this is the minimum precision level for the modal frequency error.

The fidelity of the system is estimated as the minimum fidelity across components. In this case, the SM support beams of length 1.21 and $1.18 \mathrm{~m}$ do not meet minimum fidelity requirements, therefore the fidelity of the system is zero. If these components did not exist, the system fidelity would be approximated using the next lowest fidelity component which is the $7.81 \mathrm{~m}$ beam with a fidelity of approximately 3.3 .

The modal frequency error of the system is estimated as the maximum frequency error present in the components. Again, since the $1.21 \mathrm{~m}$ and the $1.18 \mathrm{~m}$ beams do not meet the minimum fidelity requirement, a system modal frequency error is not calculable. The recommendation based on the fidelity results is to refine the finite element models for the $1.21 \mathrm{~m}$ and $1.18 \mathrm{~m}$ beams. If these beams weren't in the model, the system modal frequency error would be the next highest modal frequency error which is approximately $0.3 \%$ due to the optical bench. This value will be used in the next section to estimate the error in the output figures of merit.

\subsubsection{Output figure of merit error estimation}

Finally, the error in the output figures of merit is estimated using the techniques described in Section 3.5 and the modal frequency error of the system. In this section, the error in the RMS line-of-sight jitter (LOS) in the $\mathrm{x}$ - and $\mathrm{y}$-directions, is chosen as the OFM. The LOS jitter describes how off-center the image is in the image plane and is estimated using the displacements of the three mirrors and is described in Section 2.3.3.

Using sensitivity analysis, the relative change in the LOS with respect to each system modal frequency, shown in Eqn. 3.28 is calculated. The modal frequency error of $0.3 \%$ calculated in the last section is then 


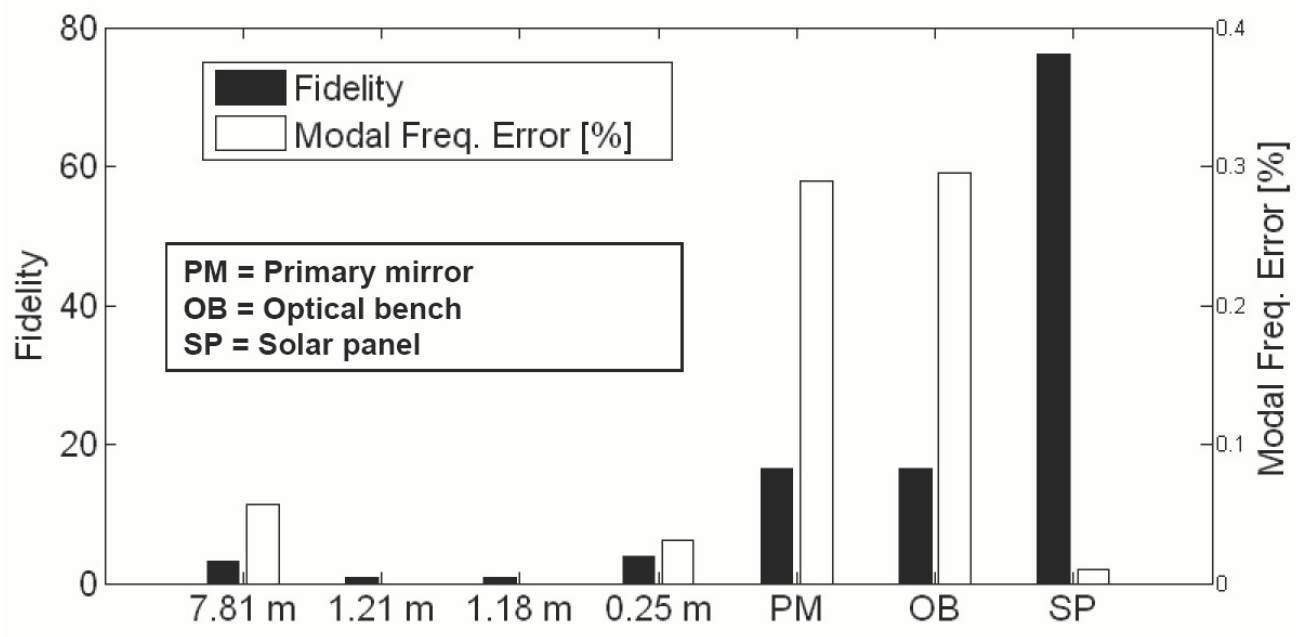

Figure 3-24. Fidelity summary for MOST components

used in Eqn. 3.29 to estimate the error in the OFM.

$$
\delta \omega=0.3 \%
$$

Since the LOS OFM is measured directly as the RMS of the system outputs, the error in the LOS jitter can be estimated directly using Eqn. 3.29. Using this technique, the error in LOS estimate is

$$
\begin{aligned}
& \delta \operatorname{LOS}_{\mathrm{x}}=0.3030 \% \\
& \delta \mathrm{LOS}_{\mathrm{y}}=0.3273 \%
\end{aligned}
$$

\subsection{Summary}

In this chapter, the structural fidelity metrics are defined for general beams and plates. Furthermore the generalized relationships between modal frequency error and fidelity are defined for beams and plates. These generalized relationships are useful since they do not dependent on specific types of loading or shapes, therefore they are applicable to a range of systems.

The fidelity assessment method, which quantifies the appropriateness of an existing complex model using the Nyquist theorem, is also described in this chapter. This method is applied to a complex telescope structural model. 
The fidelity metrics developed in this chapter can also be used to appropriately size FE meshes for model synthesis. This is described in the next chapter. 


\section{Chapter 4}

\section{Nyquist Fidelity Method}

In this chapter, the Nyquist fidelity method for model synthesis is described and implemented. The two case studies shown in this chapter are a simple cantilever beam problem and the MOST system.

\subsection{Model Synthesis Method}

The method for model synthesis based on the Nyquist fidelity method is shown graphically in Fig. 4-1. There are eight major steps and an option for iteration at the end. These eight steps are:

1. Model initialization: In this step, the geometric properties, material properties, and interface locations of the subsystem are defined. These decisions can be part of the design process or stipulated according to specifications. It is assumed that these properties are set prior to model assembly. Also defined in this step are the disturbance spectra. Since frequency domain analysis is assumed, disturbance spectra are required for analysis. These can be estimated from experiments or taken directly from disturbance models. The disturbance is assumed given for this process. The output figures of merit (OFM) are also chosen in this step.

2. Important subsystem identification: The influential subsystems are identified in this step. This is accomplished by decomposing the system into subsystems and then observing the flow of the disturbance throughout the system. Similar to the fidelity assessment method, subsystems in the direct path between the disturbance and the location of the OFM measurement are deemed influential and 
therefore are considered important.

3. Choice of modal frequency error (MFE): In this step, a MFE between $0.01 \%$ and $10 \%$ is chosen by the modeler. This corresponds to the maximum amount of modal frequency error tolerated in each subsystem component due to under-sampling of the FEM.

4. Calculate fidelities: Using the MFE and the previously derived relationships for beams and plates, required fidelity levels for model components in the important subsystems are obtained.

5. Calculate mesh size: Using the required fidelities for each component and the highest frequency in the disturbance spectra, the mesh size for each component is calculated. This mesh size is sufficiently small such that the error in the modal frequency of each component is equal to or less than the value chosen in Step 3.

6. Assemble IM model: In this step, the state space integrated model is created from the FEM of the structure with sufficient mesh size and the control systems, if any. This model defines a relationship between the input disturbance and the OFM.

7. Run analyses: The frequency domain disturbance analysis is performed in this step on the IM, giving an OFM estimate.

8. Estimate OFM error: By running a sensitivity analysis on the IM, an a posteriori error estimate of the OFM is obtained.

The eighth step is not necessary for model synthesis, but it does estimate the OFM error based on the expected modal frequency error.

After the eighth step, if the OFM estimated error is too high, a lower MFE can be chosen, and steps 3 through 8 can be repeated until an adequate MFE is obtained.

The beam problem, described in the next section, demonstrates this process in detail and serves as an in-depth description of the method. The MOST system application, presented in Section 4.3, shows how this method is applied to more complex systems. In both case studies, the computational time required for this method is tracked. Time savings and accuracy afforded by the Nyquist fidelity method compared to the model reduction method are shown in both cases. A graphical representation of the differences 


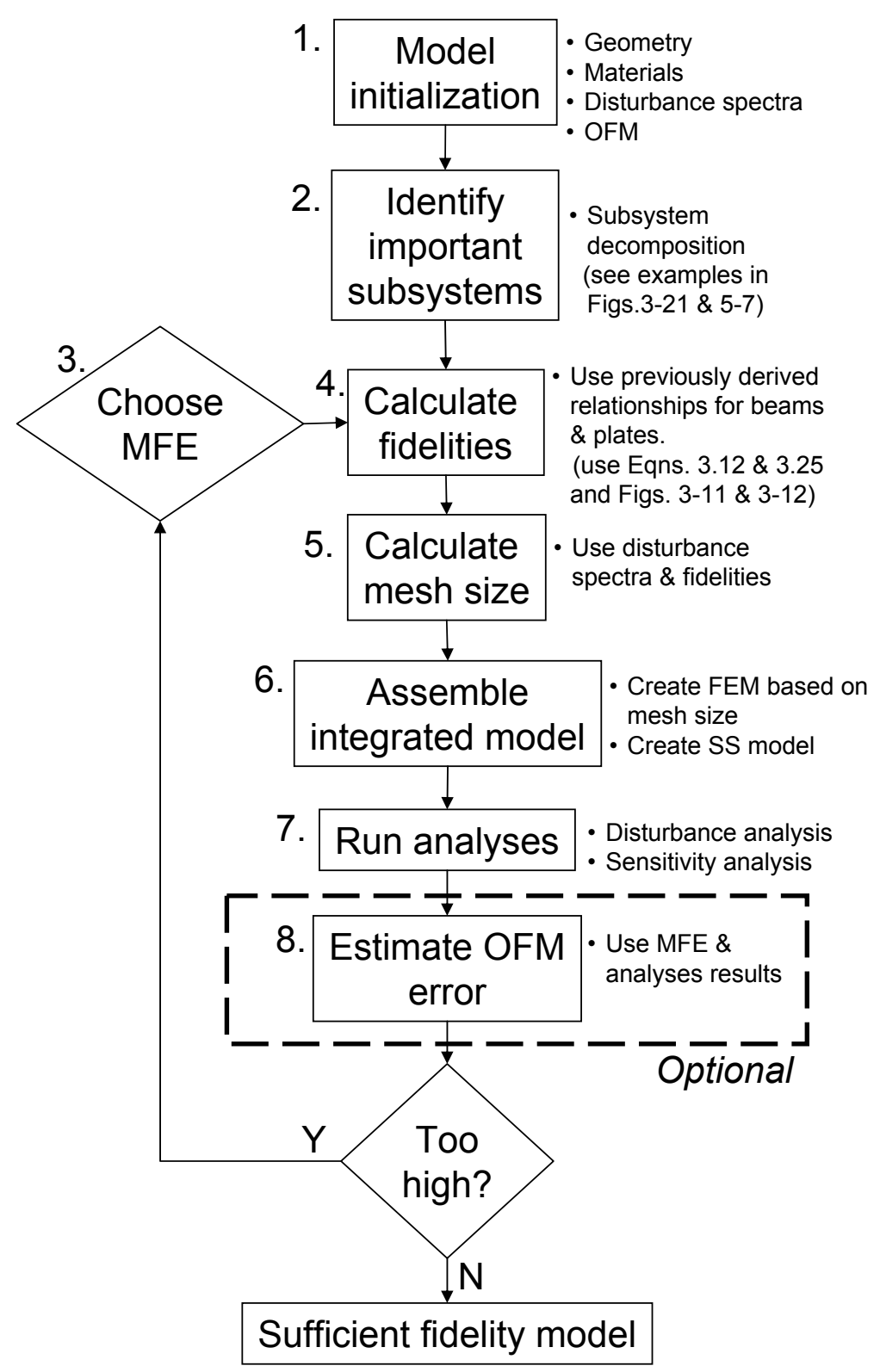

Figure 4-1. Model synthesis using the Nyquist fidelity method 


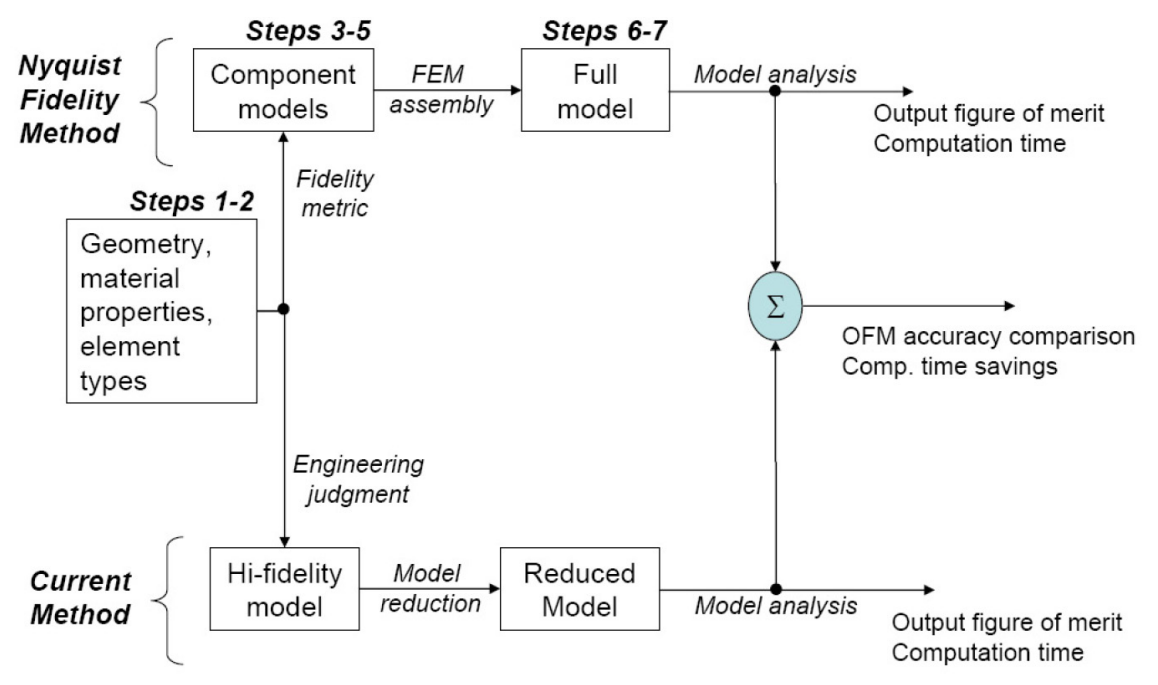

Figure 4-2. Methods for developing structural models

of these two methods can be seen in Fig. 4-2. The major difference between these methods is that in the model reduction method, the initial high-fidelity model is created and meshed based on engineering judgement. In the Nyquist method, while engineering judgement is used, the measurement of fidelity is also used as a sufficiency guideline to suggest minimum mesh sizes. Another difference is that in the model reduction method, the initial model, which is usually computationally expensive due to fine meshing, is reduced modally. In the Nyquist method, the model contains sufficiently small mesh sizes to achieve a specified modal frequency error and is therefore generally not as computationally expensive as the highfidelity model. Although the Nyquist model can be modally reduced to further reduce the time required for disturbance analysis, it is shown in this chapter without the model reduction step to highlight the difference.

Comparisons between the Nyquist fidelity method and adaptive mesh methods are not made here since current mesh refinement techniques are used often in the detailed design phase where the configuration is set and very high accuracies are needed. This thesis focuses on systems in the conceptual design phase.

\subsection{Beam Sample Problem}

In order to demonstrate the implementation of the Nyquist fidelity method, a simple sample problem is shown in this section. The sample problem consists of an isotropic, homogenous cantilever beam with a constant cross section and a loading force at the tip, shown in Fig. 4-3. This system is constructed using the 


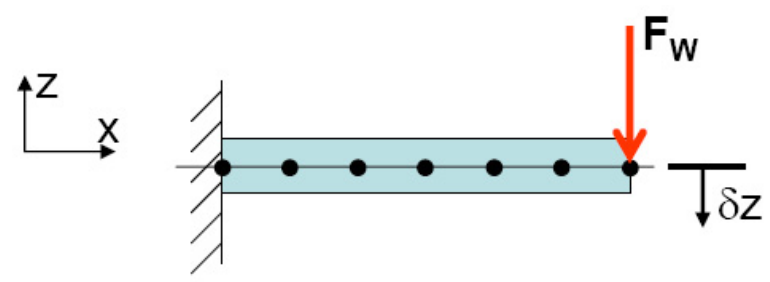

Figure 4-3. Beam sample problem

Nyquist fidelity method and using the balanced reduction method described in Section 2.2.2. The accuracies and computation times of each method are compared.

\subsubsection{Beam model synthesis using Nyquist fidelity method}

The first step in the Nyquist fidelity method is to initialize the model. The system geometry can be seen in Fig. 4-3 and the material and geometric properties are listed in Table 4.1. These properties are similar to a 6000 series aluminum alloy. The disturbance spectra must also be identified in this step. Two different band-limited disturbances are used in this example, both with an upper frequency of $1000 \mathrm{~Hz}$. Their spectra, shown as power spectral densities (PSDs), can be seen in Fig 4-4 The first disturbance is a band-limited white noise, with a forcing magnitude of $1000 \mathrm{~N}$ over a bandwidth between $0.1 \mathrm{~Hz}$ and $1000 \mathrm{~Hz}$. The second disturbance is termed "colored white noise" and is defined over the same bandwidth but it contains non-uniform forcing magnitudes. This spectra is created by passing the bandlimited white noise through a second order low pass filter. The corner frequency for this filter is $10 \mathrm{~Hz}$. The output figures of merit are also chosen in this step. In this case, the mean-square value of the tip displacement is the chosen output figure of merit. The tip displacement is labeled as $\delta z$ in Fig. 4-3 and the mean square tip displacement is denoted as $\sigma_{z}^{2}$

The next two steps are to identify the important subsystems and to choose a modal frequency error. In this case, there is only one subsystem, the beam. The modal frequency error is chosen to be $0.1 \%$. Using this MFE, the required fidelity of this beam is calculated using Eqn. 3.12, Rearranging Eqn. 3.12, the required 
Table 4.1. Geometric and material properties for beam sample problem

\begin{tabular}{rrr}
\hline \hline Geometric properties & Units & Values \\
\hline length & {$[\mathrm{m}]$} & 1 \\
diameter & {$[\mathrm{m}]$} & 0.1 \\
& & \\
\hline \hline Material properties & Units & Values \\
\hline Young's modulus & {$[\mathrm{Pa}]$} & $7.00 \mathrm{E}+10$ \\
Poisson's ratio & {$[-]$} & 0.3 \\
Density & {$\left[\mathrm{kg} / \mathrm{m}^{3}\right]$} & 2700 \\
\hline \hline
\end{tabular}

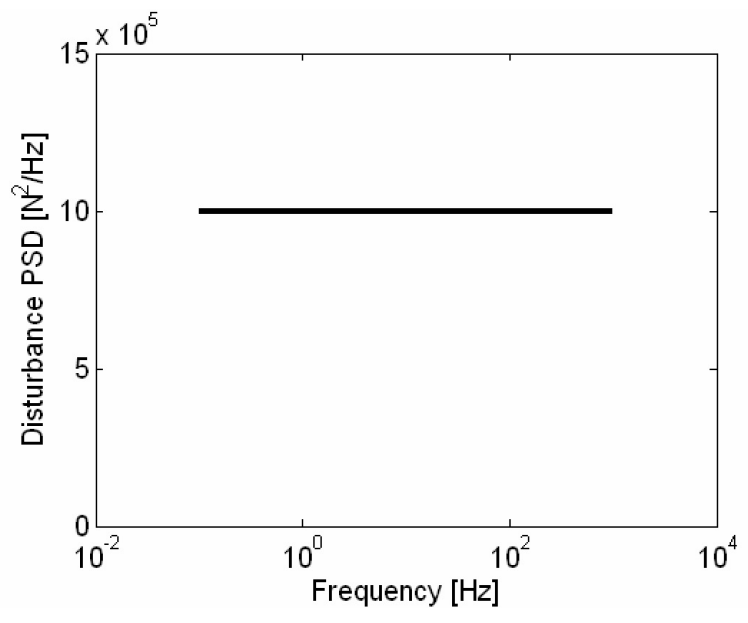

Band-limited white noise PSD

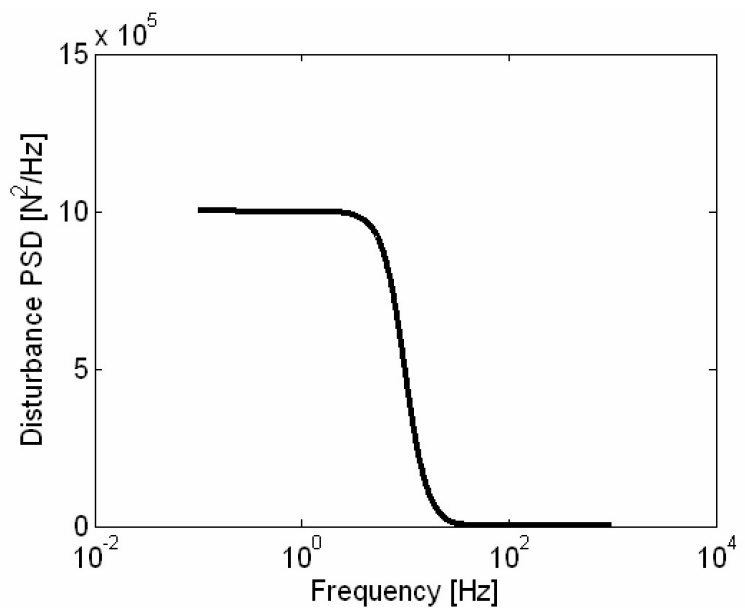

Colored white noise PSD

Figure 4-4. Disturbance power spectral density functions for beam sample problem 
fidelity is calculated as:

$$
\begin{aligned}
\mathrm{F}_{\lambda} & =\left[10^{-0.7579} \Delta \omega\right]^{-1 / 3.907} \\
& =\left[10^{-0.7579}(0.1)\right]^{-1 / 3.907} \\
& =2.82
\end{aligned}
$$

The fifth step is to calculate the mesh size. Recall that the fidelity metric is the mesh spatial frequency, $n$, divided by the Nyquist required frequency $n_{N y}$,

$$
F_{\lambda}=\frac{n}{n_{N y}}
$$

Given the material properties shown in Table 4.1 and the wave equation given in Eqns. 3.4 and 3.5 , the spatial frequency corresponding to the upper disturbance frequency of $1000 \mathrm{~Hz}$ can be calculated as,

$$
\varpi=\frac{1}{2 \pi}\left[\frac{\omega_{\max }^{2} \rho A}{E I}\right]^{1 / 4}=1.12[\text { nodes } / \mathrm{m}]
$$

where the upper disturbance frequency is,

$$
\omega_{\max }=2 \pi(1000) \mathrm{rad} / \mathrm{s}
$$

Multiplying this spatial frequency by two gives the Nyquist frequency.

$$
n_{N y}=2 \varpi=2.24[\text { nodes } / \mathrm{m}]
$$

The required mesh spatial frequency is calculated by rounding up to the next integer. This yields,

$$
n=\left\lceil F_{\lambda}\left(n_{N y}\right)\right\rceil=7.00[\text { nodes } / \mathrm{m}]
$$

Therefore, a $1 \mathrm{~m}$ beam with sufficient fidelity to produce a $0.1 \%$ MFE has 7 equally spaced nodes. This beam model, therefore, contains 6 beam elements and the mesh size, $\delta x$, is the length divided by the number of elements,

$$
\delta x=\frac{1}{n-1}=\frac{1}{6}[\mathrm{~m}]
$$


The nodes are spaced at $0.1667 \mathrm{~m}$ intervals.

The next step is to assemble the state space integrated model. In this case, there are no controllers and the performance metric is the displacement of the node at the tip. Therefore, the state space model is the second order modal form of the structural model, constructed from the modal frequencies, $\Omega$, and modeshapes, $\Phi$,

$$
\begin{aligned}
& \dot{x}=A x+B u \\
& z=C x
\end{aligned}
$$

where

$$
\mathrm{A}=\left[\begin{array}{cc}
0 & \mathrm{I} \\
-\Omega^{2} & -2 \zeta \Omega
\end{array}\right], \mathrm{B}=\left[\begin{array}{c}
0 \\
\Phi^{\mathrm{T}} \mathrm{B}_{0}
\end{array}\right], \mathrm{C}=\left[\mathrm{C}_{0} \Phi\right]
$$

By selecting input and output nodes, the state space input and output matrices, $B_{0}$ and $C_{0}$, are created. The input and output nodes in this case are collocated at the tip of the beam. The disturbance enters the system at the tip and the performance (mean-square tip displacement) is measured at the tip.

Next, the disturbance analysis is performed. Since the disturbances are band-limited, the PSD integration method, described in Section 2.2.1 is used. If the disturbances are defined over all frequencies, the Lyapanov method would be used since it is usually less computationally expensive than the PSD integration method. In the case of band-limited disturbance, the Lyapanov analysis still could be used. However, the white noise shaping filter required to represent a band-limited disturbance accurately over a large bandwidth generally is prohibitively large to avoid ripple (in the Chebyshev cases), or to represent steep descent to zero at the upper frequency (Butterworth filters). The PSD integration method is therefore more appropriate in this case. The disturbance analysis calculates the mean square of the tip displacement, $\sigma_{z}^{2}$. The mean-square tip displacements calculated using this model are $2.0007 \mathrm{E}-3 \mathrm{~m}$ and $2.2468 \mathrm{E}-5 \mathrm{~m}$ for the white noise and colored noise cases, respectively. These values are also listed in Table 4.3 .

Finally, the error in the OFM due to under-sampling is estimated using the form of Eqn. 3.29 . In this case, however, the OFM is the mean-square displacement value and not the root-mean-square displacement. The estimated error bound of the mean-square tip displacement is calculated as,

$$
\delta \sigma_{z}^{2}=\sum_{k=1}^{m}\left|\frac{\% \text { change in } \sigma_{z}^{2}}{\% \text { change in } \omega_{k}}\right|(\delta \omega)
$$


where $m$ is the number of modes in the system below $1000 \mathrm{~Hz}$ and $\delta \omega$ is the modal frequency error chosen in step 3 of $0.1 \%$. Table 4.2 shows that there are two modes below $1000 \mathrm{~Hz}$, therefore $m$ is two.

In order to estimate this error, the relative sensitivities must first be calculated. The sensitivity analysis presented in Section 2.2.3 calculates the change in the RMS tip displacement with respect to the change in the system modes, $\frac{\partial \sigma_{z}}{\partial \omega_{k}}$, where $k$ is the index to each system mode. Therefore, the sensitivity of the OFM (mean-square tip displacement) with respect to the system modes is

$$
\frac{\partial \sigma_{z}^{2}}{\partial \omega_{k}}=\frac{\partial \sigma_{z}}{\partial \omega_{k}}\left(2 \sigma_{z}\right)
$$

Using Eqn. 3.28, the relative sensitivities for each mode are calculated as

$$
\frac{\% \text { change in } \sigma_{z}^{2}}{\% \text { change in } \omega_{k}}=\frac{\partial \sigma_{z}^{2}}{\partial \omega_{k}} \frac{\omega_{k, n o m}}{\sigma_{z, n o m}^{2}}=\frac{\partial \sigma_{z}}{\partial \omega_{k}}\left(2 \sigma_{z}\right) \frac{\omega_{k, n o m}}{\sigma_{z, n o m}^{2}}
$$

Listed in Table 4.2 are the relative sensitivity values associated with each system mode, $\omega_{k}$, for the first ten modes. The blank entries correspond to modes with zero sensitivity to the calculable precision of less than 1E-30. The relative sensitivities for the white noise and colored white noise cases are also shown graphically in in Fig. 4-5. A steep drop off in OFM sensitivities for the white noise case can be seen around $1000 \mathrm{~Hz}$ because only the system modes below $1000 \mathrm{~Hz}$ are the major modal contributors to the OFM. In the colored white noise case, there is no steep drop-off. The sensitivities above $1000 \mathrm{~Hz}$ are non-zero because a change in their frequencies could either (1) enter the bandwidth under $1000 \mathrm{~Hz}$ or (2) influence the shape of the transfer function within the bandwidth.

Also listed in Table 4.2 are the modal frequency errors (MFE[\%]) associated with each mode. These are not individual contributions to the overall modal frequency error, rather they show the modal frequency error present in the system if all modes up to that mode were within the bandwidth of interest. So, for example, if mode 3 were included in the bandwidth of interest, the system modal frequency error would be about $0.16847 \%$. Since only modes 1 and 2 are within the bandwidth of interest, the MFE present in the system is $0.02387 \%$. This meets the modal frequency error bound of $0.1 \%$ chosen earlier.

The estimated mean-square displacement errors are calculated using the values in Table 4.2 and Eqn. 4.2 . These values are $0.300 \%$ and $0.415 \%$ for the white and colored noise cases respectively, and are also listed 
Table 4.2. Sensitivity analysis values for beam sample problem with $0.1 \%$ desired modal frequency error

\begin{tabular}{lcc|ccc}
\hline \hline Mode No. & 1 & 2 & 3 & 4 & 5 \\
\hline Freq. [Hz] & 70.967 & 435.39 & 1183.9 & 1276.6 & 2240.2 \\
White noise relative sensitivity & $-2.99 \mathrm{E}+00$ & $-1.31 \mathrm{E}-02$ & $-8.80 \mathrm{E}-05$ & - & $-5.48 \mathrm{E}-05$ \\
Colored noise relative sensitivity & $-4.05 \mathrm{E}+00$ & $-9.60 \mathrm{E}-02$ & $\mathbf{- 1 . 2 8 E - 0 2}$ & - & $-3.60 \mathrm{E}-03$ \\
Fidelity & 10.07 & 4.07 & 2.47 & 2.37 & 1.79 \\
MFE [\%] & $\mathbf{0 . 0 0 0 6 9}$ & $\mathbf{0 . 0 2 3 8 7}$ & 0.16847 & 0.19519 & 0.58558 \\
& & & & & \\
\hline \hline Mode No. & 6 & 7 & 8 & 9 & 10 \\
\hline Freq. [Hz] & 3556.5 & 3917.6 & 4926.9 & 6822.4 & 8995.6 \\
White noise relative sensitivity & $-2.33 \mathrm{E}-05$ & - & $-8.95 \mathrm{E}-06$ & - & $-2.25 \mathrm{E}-06$ \\
Colored noise relative sensitivity & $-1.43 \mathrm{E}-03$ & - & $-5.40 \mathrm{E}-04$ & - & $-1.35 \mathrm{E}-04$ \\
Fidelity & 1.42 & 1.36 & 1.21 & 1.03 & $\mathbf{0 . 8 9}$ \\
MFE [\%] & 1.4445 & 1.7449 & 2.7305 & 5.157 & $\mathbf{8 . 8 5 1 2}$ \\
\hline \hline
\end{tabular}

in parentheses in Table 4.3 .

As a comparison, if all of the system modes 1 are included in the summation in Eqn. 4.2 , then

$$
m=18
$$

and the mean-square displacement error values are then $0.300 \%$ and $0.417 \%$. This increase from $0.415 \%$ to $0.417 \%$ for the colored white noise case is due primarily to the relatively large sensitivity value of the third mode (boldface in Table 4.2). This mode is $1184 \mathrm{~Hz}$ and is close to the upper disturbance frequency of 1000 Hz.

For demonstration purposes, the fidelity levels and corresponding modal frequency errors for each mode are also shown in Table 4.2. Since this system consists entirely of a beam, the fidelity and modal frequency error can be calculated for each mode. The fidelity levels are calculated for each mode by using twice each mode's spatial frequency as the denominator of the fidelity metric in Eqn 3.2 .

$$
F_{\lambda, \omega_{k}}=\frac{n}{n_{N y}}=\frac{n}{2 \varpi_{F E M, \omega_{k}}}
$$

where $\omega_{k}$ is the $k^{t h}$ modal frequency, and $\varpi_{F E M, \omega_{k}}$ is the spatial frequency based on the FEM-calculated time-domain modal frequency, $\omega_{k}$. The expected modal frequency errors for each mode are calculated using

\footnotetext{
${ }^{1}$ There are a total of 18 system modes, since 6 nodes in the beam model each have 3 degrees of freedom.
} 


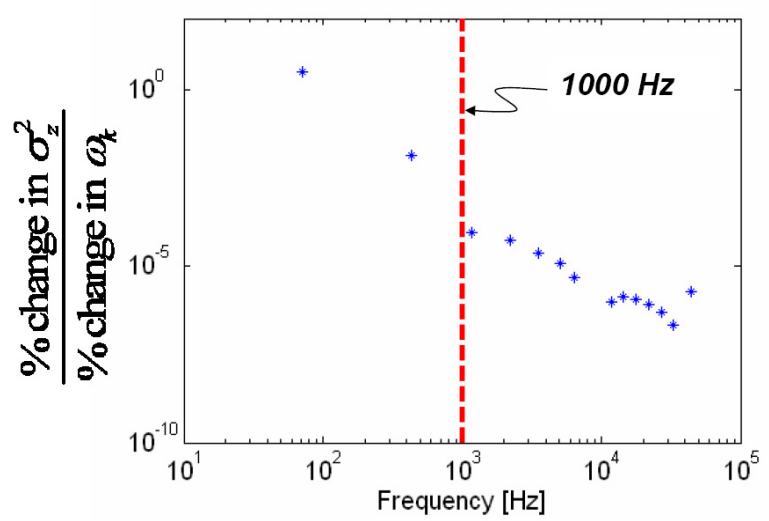

(a) Band-limited white noise PSD

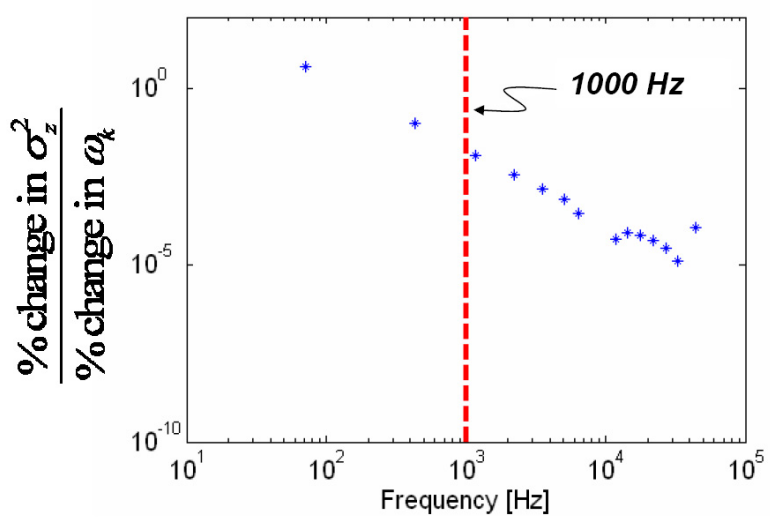

(b) Colored white noise PSD

Figure 4-5. Sensitivity of OFM with respect to the modal frequencies

the Generalized Beam Eqn. 3.12 and the fidelity level for each mode. The tenth mode is of particular interest, since its fidelity is less than the minimum required level of 1.0. This indicates that above the ninth mode, the modeshapes are aliased. Furthermore, this indicates that displacement values derived from modeshapes ten and higher do not approach the true value and are therefore inaccurate.

\subsubsection{Balanced reduction method}

The balanced reduction method is one of the current state of the art methods for frequency domain analyses of large complex systems. The procedure for this method is described in Section 2.2.2. The beam sample problem is modeled using this method and the accuracy of the OFM and the computation times are compared to that of a model created using the Nyquist fidelity method.

In the balanced reduction method, the beam is modeled using 100 elements per meter. Once the finite element model is created, the finite element analysis is performed, giving the natural frequencies, $\Omega$, and the modeshape matrix, $\Phi$. The modes and modeshapes are then assembled into a state space model, the same way as in the Nyquist fidelity method. As shown in Section 2.2.2, the RMS error between the full and reduced models can be chosen by the user in order to truncate the appropriate number of modal states (Eqn. 2.13). This is a guaranteed error only when using the Lyapanov analysis. Since PSD integration analysis is used here, this chosen error is an estimate. An RMS tip displacement error of $0.15 \%$ is chosen, 
giving a mean-square tip displacement error of $0.3 \%$ since

$$
\partial \sigma_{z}^{2}=2 \partial \sigma_{z}
$$

This mean-square tip displacement error is equal to the error calculated in the Nyquist fidelity method for the white noise case. It was chosen in order to compare the two methods by setting the accuracy estimates equal to each other. The model is truncated accordingly and the disturbance analysis is performed again using the PSD integration method. The results of both the balanced reduction and Nyquist fidelity methods can be seen in Table 4.3 ,

Table 4.3. Disturbance analysis results for beam sample problem

\begin{tabular}{rccc}
\hline \hline & Full Model & Reduced Model & Nyquist Fidelity Method \\
\hline \# elements & 100 & 100 & 6 \\
\# states & 720 & 6 & 18 \\
\hline \multicolumn{5}{c}{ Band-limited White Noise Disturbance } \\
\hline$\sigma_{z}^{2}$ & $2.0004 \mathrm{E}-03$ & $2.0004 \mathrm{E}-03$ & $2.0007 \mathrm{E}-03$ \\
$\sigma_{z}^{2}$ error [\%] & - & 0.0025 & 0.0107 \\
Estimated error bound [\%] & - & $0.3 \%$ & $0.3 \%$ \\
\hline \hline \multicolumn{5}{c}{ Band-limited Colored White Noise Disturbance } \\
\hline$\sigma_{z}^{2}$ error [\%] & $2.2467 \mathrm{E}-05$ & $2.2397 \mathrm{E}-05$ & $2.2468 \mathrm{E}-05$ \\
Estimated error bound [\%] & - & 0.3077 & 0.0085 \\
\hline \hline
\end{tabular}

\subsubsection{Comparison}

A comparison of the system transfer functions can be seen in Figure 4-6. The 100-element beam is generated in the same way as the balanced reduction model, but no reduction step is taken. It is shown here for comparison purposes. The first three modes match well for all three models. It can be seen that above the third mode at $1183.9 \mathrm{~Hz}$, the reduced model has no more modal content and a steep roll-off. The Nyquist fidelity model tracks the full model well until about $3.2 \mathrm{kHz}$. At about $45 \mathrm{kHz}$, the Nyquist fidelity model seems to pop-up above the 100-element model. It is not clear why this happens. Less effort is required to create the Nyquist fidelity model of 6 beam elements than the reduced model which requires the 100-element beam model to be created and then reduced. However, the Nyquist fidelity method produces a model that 


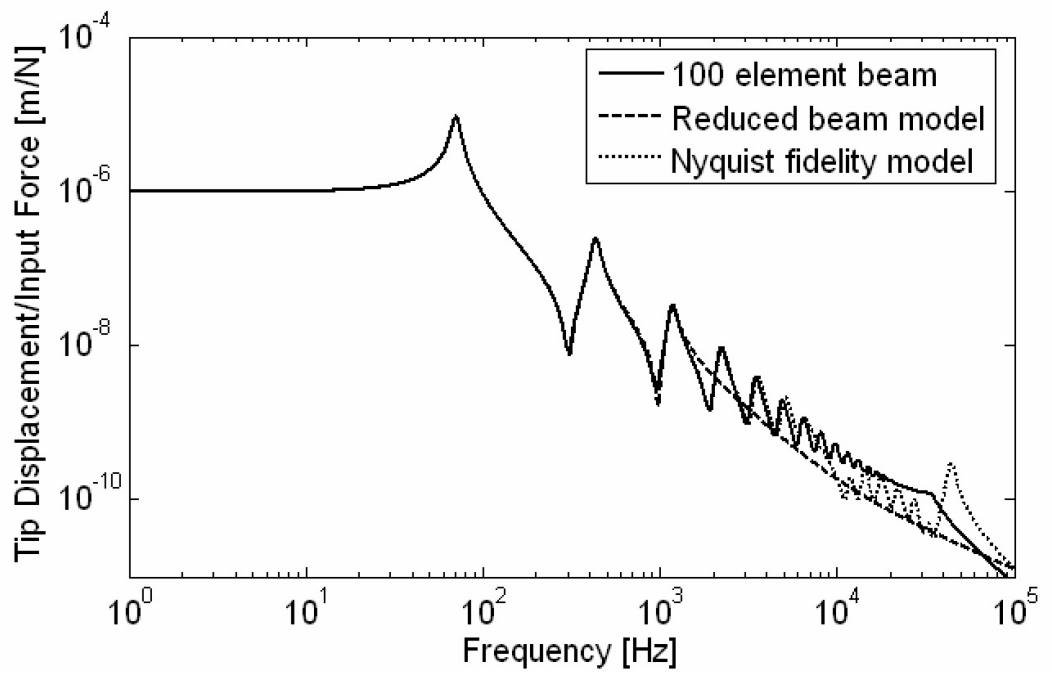

Figure 4-6. Beam model system transfer functions

tracks the full 100-element model over a greater bandwidth.

A comparison of the output PSDs can also be made. Using the PSD disturbance analysis, the output PSD for both disturbances can be generated for comparison purposes. These output PSDs are shown in Fig. 4-7. It can be seen that within the disturbance bandwidth of 0.1 to $1000 \mathrm{~Hz}$, the output PSD of the tip displacements overlap almost exactly. This indicates that all three models calculate approximately the same value for the OFM, since the OFM is calculated by integrating these PSDs over the disturbance bandwidth. If the 100-element beam model is taken to be the "truth" model, the overlap of the output PSDs indicate that there is very little OFM error in both methods. The next section examines these errors.

\subsubsection{Results}

The disturbance analysis results can be seen in Table 4.3 for the white noise and colored white noise cases. The accuracies of the output metric estimate, $\sigma_{z}^{2}$, are measured as the percent error compared against the full 100-element unreduced model. These accuracies are labeled " $\sigma_{z}^{2}$ error". Also listed in Table 4.3 are the estimated error bounds for each modeling method and each disturbance case. It can be seen that the percent errors fall below the error bounds in all cases except for the model reduction case with colored white noise disturbance. In this case, it only exceeds the bound by a small amount. $2^{2}$

\footnotetext{
${ }^{2}$ This is because the error chosen in the model reduction method, here $0.3 \%$ mean-square displacement error, is guaranteed only for the Lyapanov analysis. Since this disturbance is bandlimited, the PSD integration analysis is used, and the actual error can be
} 


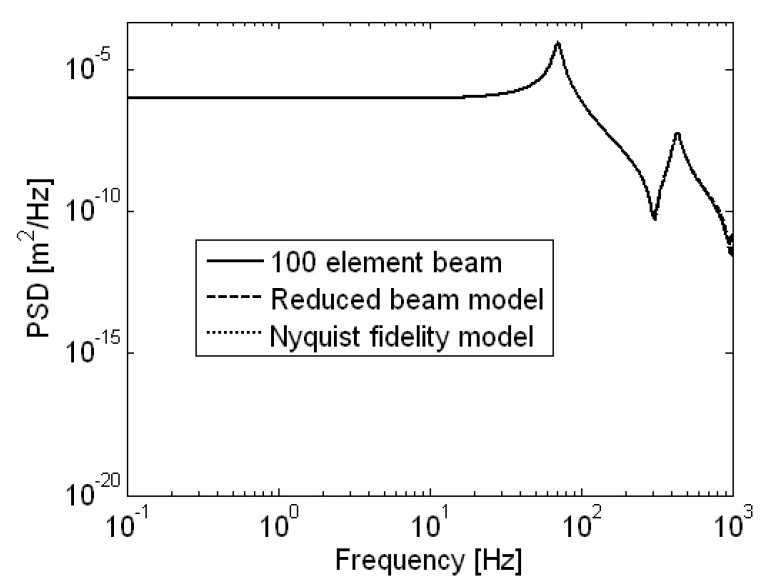

Input: band-limited white noise

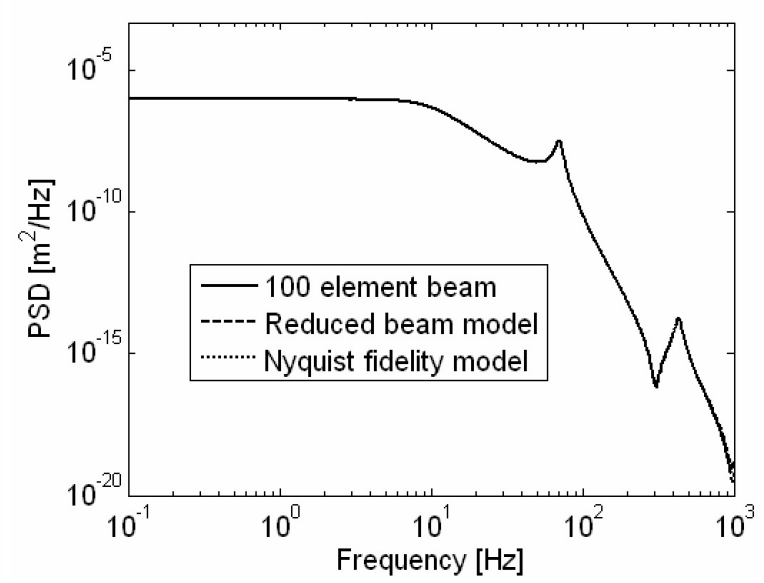

Input: colored white noise

Figure 4-7. Tip displacement output PSDs

Listed in Table 4.4 are the average calculation times for both methods broken out by the different steps needed in each method. Each analysis is performed ten times and the averages are calculated. Also shown in Table 4.4 are the $95 \%$ confidence intervals for each calculation time. These indicate with $95 \%$ confidence that the calculation times are random samples of a normal distribution and that the true mean the distribution falls within that interval [63]. Since all the average times fall within their respective intervals, we have confidence that the average times are good indicators of computational expense. The average times are also displayed graphically in Fig. 4-8. Not including the optional error estimation calculation, the Nyquist fidelity method provides approximately a $40 \%$ reduction in calculation time over the model reduction method for the white noise disturbance and colored noise cases for this example. The calculation time of the model reduction method includes the construction of the 100-element finite element model (assembly of stiffness and mass matrices), the finite element normal modes analysis, the model reduction and the disturbance analysis on the reduced model. The calculation time for the Nyquist fidelity method includes the construction of the 6-element finite element model, the finite element normal modes analysis, and the the disturbance analysis. Including the optional error estimation calculation, the Nyquist fidelity method still provides roughly a $25 \%$ reduction in computation time for both disturbance analyses with no loss in accuracy relative to the required error bound.

different from the set error. 
Table 4.4. Average calculation times for beam sample problem

\begin{tabular}{|c|c|c|c|c|}
\hline \multicolumn{5}{|c|}{ Band-limited White Noise Disturbance } \\
\hline & Mod Red & 95\% Confidence Interval & Nyquist & 95\% Confidence Interval \\
\hline Build & 4.2231 & $(4.0728,4.3734)$ & 2.6622 & $(2.5840,2.7404)$ \\
\hline Reduce & 0.3215 & $(0.3156,0.3274)$ & - & - \\
\hline Dist & 0.1588 & $(0.1540,0.1636)$ & 0.1628 & $(0.1568,0.1688)$ \\
\hline Error & - & - & 0.7313 & $(0.7259,0.7367)$ \\
\hline Total & 4.7034 & $(4.5517,4.8551)$ & 3.5563 & $(3.4769,3.6357)$ \\
\hline \multicolumn{5}{|c|}{ Band-limited Colored White Noise Disturbance } \\
\hline & Mod Red & 95\% Conf. Int. & Nyquist & 95\% Conf. Int. \\
\hline Build & 4.2160 & $(4.1054,4.3266)$ & 2.67 & $(2.6006,2.7394)$ \\
\hline Reduce & 0.4741 & $(0.4663,0.4819)$ & - & - \\
\hline Dist & 0.1981 & $(0.1926,0.2036)$ & 0.2819 & $(0.2817,0.2821)$ \\
\hline Error & - & - & 0.689 & $(0.6890,0.6890)$ \\
\hline Total & 4.8882 & $(4.7763,5.0001)$ & 3.6409 & $(3.5714,3.7104)$ \\
\hline
\end{tabular}

It is important to note that the percent improvement in the computation time of Nyquist relative tot he model reduction method depends on the initial mesh size chose by "engineering judgement" (100-element beam).

\subsubsection{Beam sample problem summary}

For this example, the Nyquist fidelity method is shown to require $25 \%$ less computation time than the model reduction method, on average, while providing OFM accuracy within the estimated error bound. The model reduction method also provides an error bound, but it is only valid when using the Lyapanov disturbance analysis.

A major difference between the OFM errors of the two methods is that the Nyquist method bases its error estimate on analytical and numerical data gathered a priori, whereas the model reduction method bases the error as a the difference between the highly-meshed and reduced models. However, the mesh-size for a "highly meshed" model is based on engineering judgement and could in some cases not be "highly meshed" but in fact violate the Nyquist sampling criterion. 


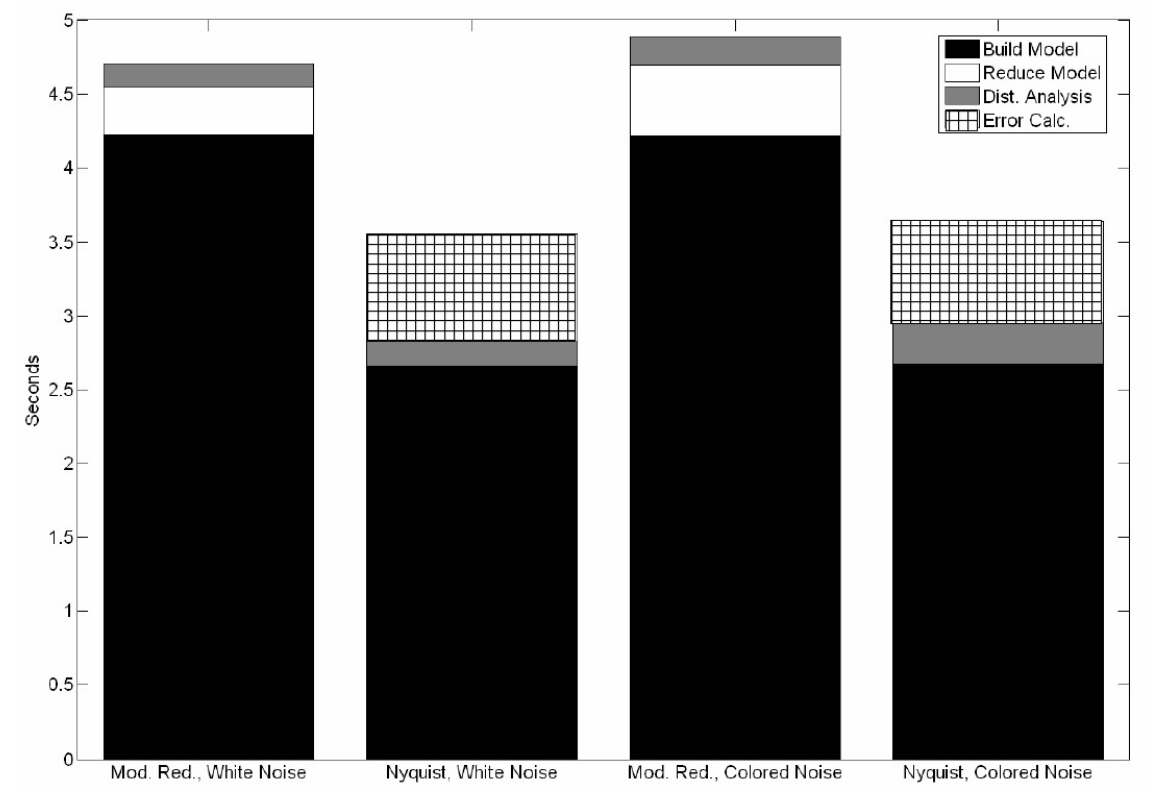

Figure 4-8. Calculation time comparison chart for beam sample problem

\subsection{MOST Model Synthesis}

In this section, the Nyquist fidelity method is applied to the MOST system, introduced in the previous chapters. The steps in the Nyquist Fidelity method are broken up into the four following subsections: Section 4.3.1 discusses steps 1-3, Section 4.3.2 shows steps 4 and 5, Section 4.3.3 shows steps 6 and 7, and Section 4.3 .4 demonstrates the 8th step.

\subsubsection{MOST Model Setup}

The first two steps in the Nyquist Fidelity method, shown in Fig. 4-1, are presented in Sections 3.7.2 and 3.7.1. Figure. 2-5 shows the nominal configuration of MOST and Table 2.2 lists the MOST nominal parameter values. The disturbance spectrum is shown in Fig. 2-9 with an upper frequency of $100 \mathrm{~Hz}$. The output figures of merit are defined as the line-of-sight (LOS) jitter and wave front error (WFE). Through the network analysis, the important components are identified as the secondary support tower (SST), the primary and secondary mirrors, the optical bench and the solar panels. For comparison purposes, two modal frequency error levels of $1.0 \%$ and $5.0 \%$ are chosen. Therefore, two models, based on these two MFE values, are developed in this Section in parallel. 


\subsubsection{Mesh Sizing}

In this section, the mesh sizes for the beams in the SST and the plates are calculated. First, the required fidelity of each component is calculated using the Generalized Beam Equation and the Generalized Plate Equation. Then, the mesh sizes are calculated based on the $100 \mathrm{~Hz}$ upper disturbance frequency.

\section{Sizing SST Beams}

First, the beams in the SST are sized. As shown in Fig. 3-22, there are beams of four different lengths in the SST: $7.18 \mathrm{~m}, 1.21 \mathrm{~m}, 1.18 \mathrm{~m}$, and $0.25 \mathrm{~m}$. Using the Generalized Beam equation, the fidelity level for all the beams for the $\delta \omega=1.0 \%$ case is

$$
\begin{aligned}
\mathrm{F}_{\lambda} & =\left[10^{-0.7579} \delta \omega\right]^{-1 / 3.907} \\
& =\left[10^{-0.7579}(1.0)\right]^{-1 / 3.907} \\
& =1.56
\end{aligned}
$$

and for the $\delta \omega=5.0 \%$ case is

$$
\begin{aligned}
\mathrm{F}_{\lambda} & =\left[10^{-0.7579} \delta \omega\right]^{-1 / 3.907} \\
& =\left[10^{-0.7579}(5.0)\right]^{-1 / 3.907} \\
& =1.04
\end{aligned}
$$

Next, the Nyquist spatial frequencies are calculated as twice the associated spatial frequency, $\varpi$, as,

$$
n_{N y}=2 \varpi
$$

and $\varpi$ is calculated using the wave equation, given in Eqn. 3.5 ,

$$
\varpi=\frac{1}{2 \pi}\left[\frac{\omega_{\max }^{2} \rho A}{E I}\right]^{1 / 4}
$$

where the upper disturbance frequency is

$$
\omega_{\max }=2 \pi(100) \mathrm{rad} / \mathrm{s}
$$


Table 4.5. Mesh sizing for MOST beams for $5 \%$ and $1 \%$ MFE

\begin{tabular}{|c|c|c|c|c|c|c|}
\hline \multicolumn{3}{|c|}{ Beam lengths [m] } & 7.81 & 1.21 & 1.18 & 0.25 \\
\hline & & $\varpi$ & 0.383 & \multicolumn{3}{|c|}{1.050} \\
\hline & & $n_{N y}$ & 0.766 & \multicolumn{3}{|c|}{2.100} \\
\hline \multirow{3}{*}{$\begin{array}{c}\text { Fidelity }= \\
\text { MFE }=\end{array}$} & 1.56 & $\mathrm{n}$ & 1.195 & \multicolumn{3}{|c|}{3.276} \\
\hline & $1.0 \%$ & nodes & 10 & 4 & 4 & 1 \\
\hline & & $\delta x[m]$ & 0.868 & 0.403 & 0.393 & 0.250 \\
\hline \multirow{3}{*}{$\begin{array}{r}\text { Fidelity }= \\
\text { MFE }=\end{array}$} & 1.04 & $\mathrm{n}$ & 0.797 & \multicolumn{3}{|c|}{2.184} \\
\hline & $5.0 \%$ & nodes & 7 & 3 & 3 & 1 \\
\hline & & $\delta x[m]$ & 1.302 & 0.605 & 0.590 & 0.250 \\
\hline
\end{tabular}

This value is different for beams with different material and cross sectional properties. These Nyquist spatial frequencies are multiplied by the fidelities to calculate the mesh spatial frequency,

$$
n=F_{\lambda}\left(n_{N y}\right)
$$

The number of nodes in each beam is

$$
\text { nodes }=\lceil n L\rceil
$$

where $L$ is the length of the beam. Recall that in the Nyquist theorem, the sample points are spaced at equal intervals. Therefore if the number of nodes is a non-integer, it is rounded up so that there is an integer number of nodes spaced at sufficiently small intervals. The size between intervals, $\delta x$, is then the length divided by of the number of elements,

$$
\delta x=\frac{L}{\text { nodes }-1}[\mathrm{~m}]
$$

These values are shown in Table 4.5 for each beam and each of the fidelity levels shown in Eqns. 4.6 and 4.6

The mesh sizes for each beam and each fidelity level are shown in Table 4.5 in the $\delta x$ rows. Note that for the shortest beam, only one node is required for both fidelity levels. Since at least two nodes are required to define a beam, the $\delta x$ length is simply the length of the beam, $0.25 \mathrm{~m}$. 


\section{Sizing Plates}

The plates sized in this section are the primary and secondary mirrors, the optical bench and the solar panels. Rearranging the Generalized Plate Equation (Eqn. 3.25),

$$
\mathrm{F}_{\lambda}=\left[\frac{\Delta \omega}{175}\right]^{-1 / 2.2808}
$$

the fidelities can be calculated for each plate and for both modal frequency error levels of $1.0 \%$ and 5.0\%. For the $1.0 \%$ error level, the fidelity for all plates is

$$
\begin{aligned}
& \mathrm{F}_{\lambda}=\left[\frac{1.0}{175}\right]^{-1 / 2.2808} \\
& =9.63
\end{aligned}
$$

and for the $5.0 \%$ level, the fidelity is

$$
\begin{aligned}
& \mathrm{F}_{\lambda}=\left[\frac{5.0}{175}\right]^{-1 / 2.2808} \\
& =4.75
\end{aligned}
$$

in Section 3.4.2, it was suggested that the minimum fidelity level for plates is 4.0 . The fidelity levels shown in Eqns. 4.8 and 4.9 meet this minimum fidelity requirement.

As in the MOST beams, the Nyquist spatial frequencies are calculated as twice the associated spatial frequency, $\varpi$, as

$$
\varpi_{N y}=2 \varpi
$$

and $\varpi$ is calculated using the wave equations 3.20 and 3.21 .

$$
\varpi=\frac{\sqrt{\omega}}{2 \pi}\left[\frac{\rho h}{D}\right]^{1 / 4}
$$

where $\omega$ is the upper disturbance frequency of $200 \pi \mathrm{rad} / \mathrm{sec}, \rho$ is the volumetric mass density, $h$ is the plate thickness and $D$ is the flexural rigidity from Eqn. 3.22 . In the solar panel case, $\omega$ is the estimated first bending frequency of the panel $(\mathrm{Hz})$ since it is an appendage. 
Using the fidelity levels and the Nyquist spatial frequencies, the nodal density is calculated as

$$
\# \text { nodes } / m^{2}=\left(\varpi_{N y} F_{\lambda}\right)^{2}
$$

and the number of nodes in each plate is calculated as

$$
\# \text { nodes }=\left\lceil\left(\# \text { nodes } / m^{2}\right) A\right\rceil
$$

where $A$ is the plate surface area. The number of nodes is rounded up to the next highest integer. These values are shown in Table 4.6

Table 4.6. MOST plate mesh sizing

\begin{tabular}{rrcccc}
\hline \hline & & $\begin{array}{c}\text { Primary } \\
\text { Mirror }\end{array}$ & $\begin{array}{c}\text { Secondary } \\
\text { Mirror }\end{array}$ & $\begin{array}{c}\text { Optical } \\
\text { Bench }\end{array}$ & $\begin{array}{c}\text { Solar } \\
\text { Panel }\end{array}$ \\
\hline & $\omega[\mathrm{rad} / \mathrm{sec}]$ & $6.28 \mathrm{E} 2$ & $6.28 \mathrm{E} 2$ & $6.28 \mathrm{E} 2$ & 0.262 \\
& $\varpi[$ cycles $/ \mathrm{m}]$ & 0.708 & 0.708 & 0.494 & 0.049 \\
& $\varpi_{N y}[$ cycles $/ \mathrm{m}]$ & 1.417 & 1.417 & 0.987 & 0.098 \\
\hline Fidelity $\mathbf{= 9 . 6 3}$ & nodes $/ \mathrm{m}^{2}$ & 186.02 & 186.02 & 90.30 & 0.89 \\
MFE $=\mathbf{1 \%}$ & nodes & 1315 & 13 & 193 & 2 \\
\hline Fidelity $=\mathbf{4 . 7 5}$ & nodes $/ \mathrm{m}^{2}$ & 45.36 & 45.36 & 22.02 & 0.22 \\
MFE $=\mathbf{5 \%}$ & nodes & 321 & 3 & 47 & 1 \\
\hline \hline
\end{tabular}

\section{MOST Sizing Summary}

The mesh sizes for the MOST model sized according to the Nyquist fidelity method are summarized in Table 4.7. These mesh sizes are used as guidelines in complex systems. Practical considerations such as connectivity and element topology are taken into consideration when sizing the final mesh. The calculated mesh sizes and the final mesh sizes for the plates, therefore, are given in Table 4.7. In the beam cases, the element size can be implemented as is since beams are one-dimensional. In the plate cases, the FE layout determines the actual number of nodes. For example, the primary mirror is divided into finite elements arranged in rings of elements as in Fig. 3-14 (a). In order to include at least 1315 nodes on the PM, at least 20 element rings are needed, which contain 1380 nodes. For 321 nodes on the PM, at least 9 element rings are needed, which contain 324 nodes. In the case of the solar panel, four connection points are needed on 
Table 4.7. MOST mesh sizes for Nyquist fidelity method

\begin{tabular}{rrcrcc}
\hline \hline & \multicolumn{3}{c}{ Mesh Size [m] } & \multicolumn{3}{c}{ Nodes } \\
\hline MFE $=1 \%$ & SST beams & Calculated & Plates & Calculated & Final \\
& $7.81 \mathrm{~m}$ & 0.868 & PM & 1315 & 1380 \\
& $1.21 \mathrm{~m}$ & 0.403 & SM & 13 & 30 \\
& $1.18 \mathrm{~m}$ & 0.393 & OB & 193 & 231 \\
& $0.25 \mathrm{~m}$ & 0.250 & SP & 2 & 36 \\
\hline MFE $=5 \%$ & SST beams & Mesh Size [m] & Plates & Calculated & Final \\
& $7.81 \mathrm{~m}$ & 1.302 & PM & 321 & 324 \\
& $1.21 \mathrm{~m}$ & 0.605 & SM & 3 & 30 \\
& $1.18 \mathrm{~m}$ & 0.590 & OB & 47 & 66 \\
& $0.25 \mathrm{~m}$ & 0.250 & SP & 1 & 36 \\
\hline \hline
\end{tabular}

the short side with one extra connection point in the middle, as can be seen in Fig. 3-23 (b). Therefore, each solar panel is meshed in the final model with six nodes on a side, giving 36 nodes total per panel. An alternate method of modeling the solar panels would be to model each as a flexible beam appendage, since the number of nodes required for the solar panel are either 1 or 2 nodes.

\subsubsection{Integrated Model and Analysis}

After the mesh is appropriately sized in the components, the next step is to build the single FEM that contains every component. The same procedure for constructing the integrated model (IM) is followed as in the fidelity assessment method. The OFM results for both MFE levels are listed in Table 4.8

Table 4.8. MOST OFM values for Nyquist fidelity method

\begin{tabular}{lccc}
\hline \hline & LOSx [mas] & LOSy [mas] & RMS WFE [nm] \\
\hline $\mathrm{MFE}=1 \%$ & 1.6491 & 3.5363 & 3.278 \\
$\mathrm{MFE}=5 \%$ & 1.7376 & 3.5146 & 3.2969 \\
\hline \hline
\end{tabular}

\subsubsection{Error Estimation}

The error estimation procedure is the same as in the beam sample problem. Sensitivity analysis is performed to estimate the the change in the OFM with respect to the change in modal frequencies. Then, the specified MFE is used to calculate the expected error bound in the OFM. For a 1\% MFE, the error bound estimates 
are

$$
\mathrm{LOS}_{\mathrm{x}} \text { error bound }=2.16 \%, \quad \mathrm{LOS}_{\mathrm{y}} \text { error bound }=2.10 \%
$$

For a MFE of $5 \%$, the error bound estimates are

$$
\mathrm{LOS}_{\mathrm{x}} \text { error bound }=10.78 \%, \quad \mathrm{LOS}_{\mathrm{x}} \text { error bound }=10.05 \%
$$

These values are also shown in parentheses in Table 4.11. The error for the WFE could not be calculated due to insufficient computer memory. This can happen in sensitivity analyses, since very large matrices are manipulated during computation.

\subsubsection{Comparison and Results}

For comparison purposes, a model of the MOST system is generated using the model reduction method, which first constructs an initial model. A highly meshed model is also generated and is treated as the "truth model." Shown in Table 4.9 are the mesh sizes of this initial model and the "truth model." The mesh sizes for the initial model reduction model are based on the nominal MOST model, containing mesh sizes based on engineering judgement. Since the truth model is meshed finer than the model reduction method's initial model and the Nyquist fidelity model, it generates the most accurate OFM values. ${ }^{3}$ It is constructed to benchmark the OFM accuracy of the Nyquist fidelity and the model reduction method.

The next step in model reduction is to truncate the model. The Hankel singular values for this model are shown graphically in Fig. $4-9$ and they indicate the controllability and observability of each state. $4^{4}$ Four different truncations are made at 10, 22, 140 and 400 states, shown as vertical lines in Fig. 4-9. As more states are included, the OFMs approach their nominal values. The number of truncation states are based on the states where there appears to be a drop-off in HSVs. This is a common approach for model reduction truncation. In Lyapanov analysis, the truncation point can be determined by setting an RMS OFM error threshold. In this case of PSD integration disturbance analysis, these four truncations are made as a substitute for comparison purposes. The OFM values for the model reduction and truth models are shown in Table 4.10. The nominal model is shown here as well; it is the unreduced initial model used in the model

\footnotetext{
${ }^{3}$ An exception to this is the optical bench which is meshed in the truth model somewhat coarser than the nominal model.

${ }^{4}$ Since this is a modal model, the states are modes.
} 
Table 4.9. MOST mesh sizes for model reduction initial model and truth models

\begin{tabular}{ccccc}
\hline \hline & \multicolumn{2}{c}{ Mesh Size [m] } & Nodes \\
\hline Model Reduction Method & SST beams & \multicolumn{3}{c}{ Plates } \\
& $7.81 \mathrm{~m}$ & 0.400 & PM & 390 \\
& $1.21 \mathrm{~m}$ & 1.210 & SM & 54 \\
& $1.18 \mathrm{~m}$ & 1.180 & OB & 153 \\
& $0.25 \mathrm{~m}$ & 0.250 & SP & 60 \\
\hline "Truth" model & SST beams & & Plates \\
& $7.81 \mathrm{~m}$ & 0.100 & PM & 1380 \\
& $1.21 \mathrm{~m}$ & 0.121 & SM & 390 \\
& $1.18 \mathrm{~m}$ & 0.118 & OB & 66 \\
& $0.25 \mathrm{~m}$ & 0.250 & SP & 100 \\
\hline \hline
\end{tabular}

Table 4.10. MOST OFM values for model reduction and truth models

\begin{tabular}{rrccc}
\hline \hline & & LOSx [mas] & LOSy [mas] & RMS WFE [nm] \\
\hline Truth model & 436 states & 1.7722 & 3.5851 & 3.5552 \\
\hline Model reduction & 10 kept states & 0.0577 & 0.1907 & 4.8367 \\
& 22 kept states & 2.0248 & 3.8852 & 2.9261 \\
& 140 kept states & 1.5282 & 3.2525 & 3.0099 \\
& 400 kept states & 1.5282 & 3.2525 & 3.0099 \\
\hline Nominal Model & unreduced & 1.5282 & 3.2525 & 3.0099 \\
\hline \hline
\end{tabular}

reduction method.

The percent errors in the OFM compared the the truth model are shown in Table 4.11 for all models. It can be seen that overall the Nyquist fidelity method provides an OFM estimate that is closer to the highly meshed truth model than the Model Reduction models. Even the unreduced Nominal model is not as accurate as the Nyquist fidelity models. This is because the initially chosen mesh size of the the nominal model used in the model reduction is insufficient.

For convenience, the estimated error bounds for the Nyquist fidelity method are also shown in Table 4.11 in parentheses. It can be seen that all the OFM accuracies for the Nyquist method fall within their respective error bound estimates, with the exception of $L O S_{x}$ for the $1 \%$ MFE case. This exception is most likely due to the ill-conditioning of the MOST integrated model. Its condition number approaches the machine epsilon, and therefore the sensitivity estimates are influenced by roundoff error. This exception could also be due to the fact that the error bound shown is not absolute but an estimate. Details on the error bound estimate can be found in Section 3.5 


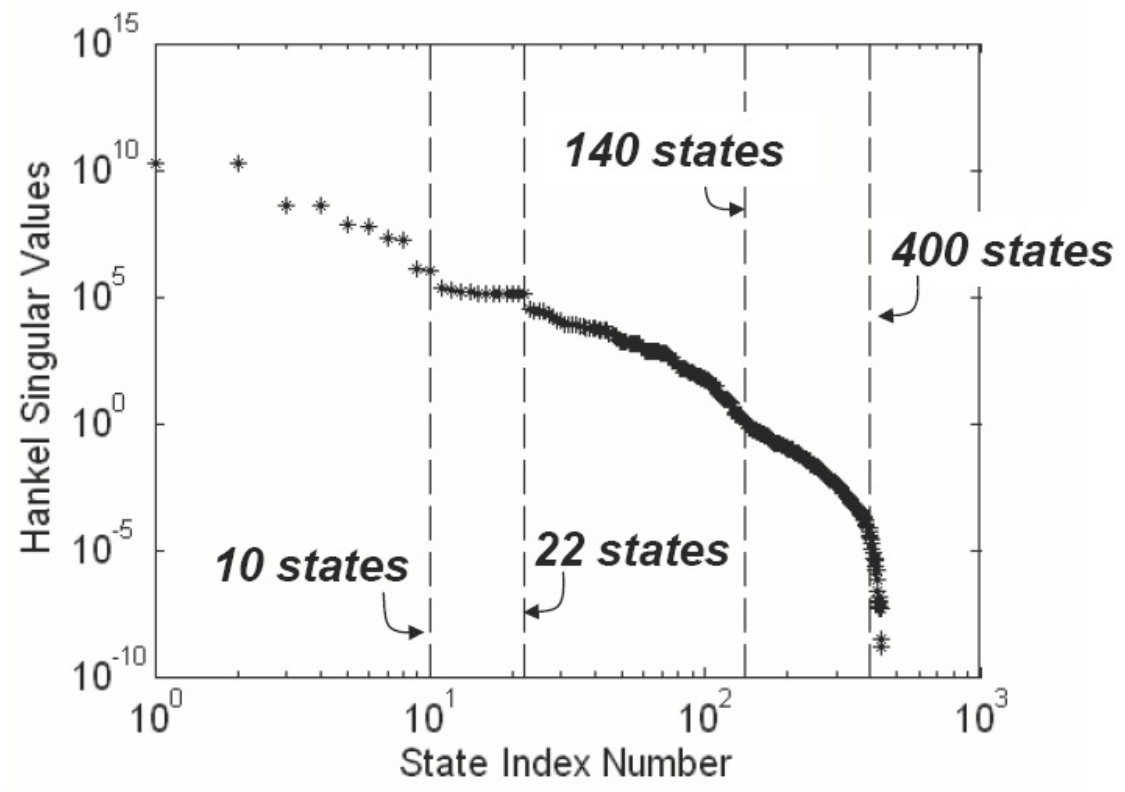

Figure 4-9. Hankel singular values

The computation times are listed in Table 4.12 and shown graphically in Fig. 4-10. It can be seen that the Nyquist fidelity method takes about $80 \%$ longer for the MFE $=1 \%$ case than the model reduction method. This is driven by the high fidelity requirements of the primary mirror and optical bench. However, the accuracy of the Nyquist method is greater. In the MFE $=5 \%$ case, about $40 \%$ less computational time is required for the Nyquist method than the model reduction method. Furthermore, since the accuracies in the Nyquist method are better than model reduction method, the model created using the Nyquist fidelity method is a more appropriate model overall.

The plot in Fig. 4-10 also decomposes the computation time according to each process. It can be seen that the disturbance analysis for some of the model reduction method cases takes less time than for the Nyquist fidelity method. However, for the finite element model (FEM) construction and system assembly take longer in the model reduction cases than in the Nyquist fidelity MFE $=5 \%$ case. Therefore, if the analysis calls for several disturbance analyses ("load cases") to be performed on one instantiation (configuration) of the system, balanced model reduction might be a better choice for a modeling method. However, if it is the case that several FEMs need to be constructed for analysis, as in the conceptual design phase where several different configurations are being investigated, than this shows that the Nyquist fidelity method can provide time savings. 
Table 4.11. Percent errors in OFM compared to the truth model

\begin{tabular}{rrccc}
\hline \hline & & LOSx [mas] & LOSy [mas] & RMS WFE [nm] \\
\hline Nyquist fidelity & MFE $=1$ & $6.95(2.16)$ & $1.36(2.10)$ & 7.80 \\
(436 states) & MFE = 5 & $1.95(10.78)$ & $1.97(10.05)$ & 7.27 \\
\hline Model reduction & 10 kept states & 96.74 & 94.68 & 36.05 \\
& 22 kept states & 14.25 & 8.37 & 17.70 \\
& 140 kept states & 13.77 & 9.28 & 15.34 \\
& 400 kept states & 13.77 & 9.28 & 15.34 \\
\hline Nominal Model & 436 states & 13.77 & 9.28 & 15.34 \\
\hline \hline
\end{tabular}

Table 4.12. Computation times comparison for MOST

\begin{tabular}{rccccc}
\hline \hline & \multicolumn{4}{c}{ Nyquist Fidelity Method } & \\
& FEM & System Assembly & Disturbance Analysis & Total \\
\hline MFE $=1$ & 139.9 & 6.9 & 14.0 & 153.7 \\
MFE =5 & 41.2 & 4.9 & 14.2 & 51.1 \\
\hline & \multicolumn{5}{c}{ Model Reduction Method } \\
& FEM & System Assembly & Model reduction & Disturbance Analysis & Total \\
\hline 10 kept states & 56.1 & 4.8 & 13.4 & 10.2 & 84.6 \\
22 kept states & 56.4 & 4.8 & 13.5 & 10.1 & 84.8 \\
140 kept states & 56.8 & 4.8 & 13.6 & 10.8 & 86.0 \\
400 kept states & 56.6 & 4.9 & 13.6 & 13.9 & 88.9 \\
\hline \hline
\end{tabular}

The computation times are plotted against the OFM percent errors in Fig. 4-11 for all models. This plot illustrates that the Nyquist fidelity method provides better accuracy over model reduction and with lower computational costs in some cases. It also shows graphically the violation of the estimated error bound for the $L O S_{x}$ OFM for the MFE $=1 \%$ case. Note also that despite a lower MFE for this data point, the MFE $=$ $5 \%$ case outperforms it for the $L O S_{x}$ OFM. This is most likely due to, again, the numerical ill-conditioning of the integrated model.

\subsection{Nyquist Fidelity Method Summary}

In this chapter, the Nyquist fidelity method for synthesizing structural models is described. Two examples of model synthesis are shown: a simple beam model and a more complex telescope model. The computational time required and the OFM accuracy of the Nyquist fidelity method are compared to that of the model reduction method for both examples. 


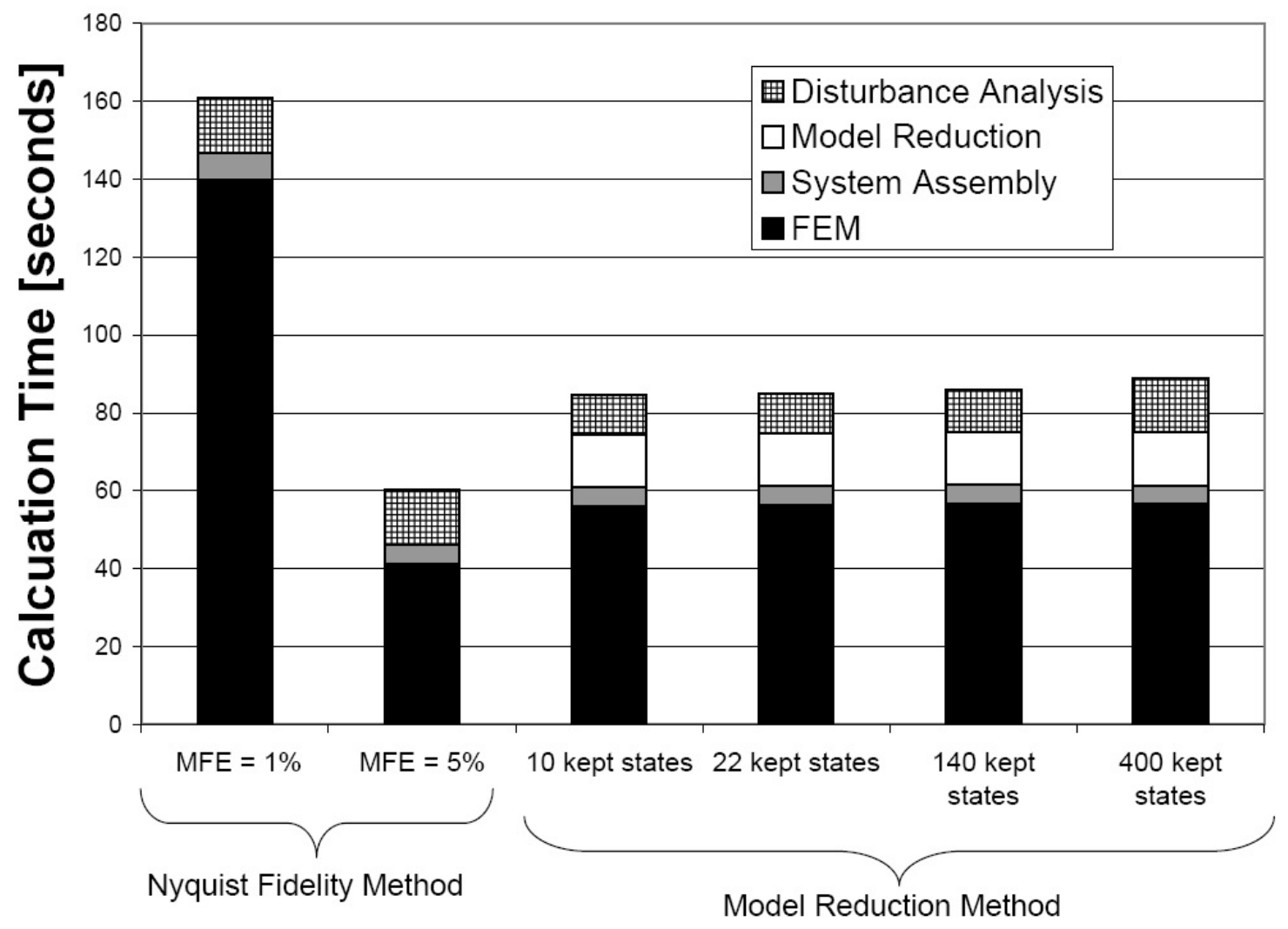

Figure 4-10. Calculation time comparison chart for MOST

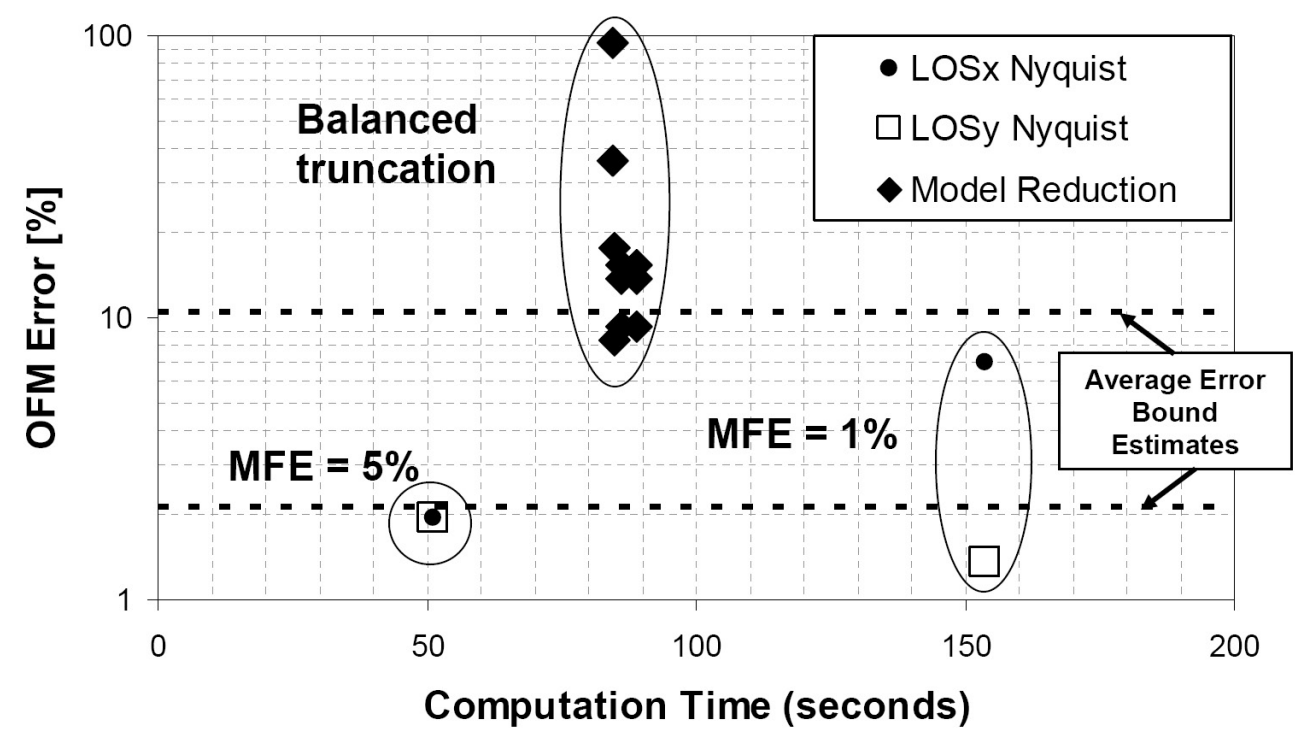

Figure 4-11. Calculation time vs. percent error for MOST 
In the beam model, it was shown that the Nyquist fidelity method requires $25 \%$ less computational time than the model reduction method while delivering accuracy within the estimated error bound. In the telescope model, the computational expense for the Nyquist fidelity method was not strictly lower than the model reduction method, however the accuracy was shown to be better in the Nyquist fidelity method.

A major benefit to using the Nyquist fidelity method over current methods is that there is no need to build a computationally expensive "truth" model to achieve reasonable accuracy results. In the model reduction method, a highly meshed model that is usually relatively computationally expensive must first be constructed. Furthermore, since the construction of this highly meshed model is based on engineering judgement, it might not contain enough fidelity to produce accurate results. The Nyquist fidelity method bases the model mesh sizes on numerical data gathered before model synthesis, and therefore does not strictly require a highly meshed "truth" model. 


\section{Chapter 5}

\section{Application to Complex Telescopes}

In this chapter, two case studies demonstrating the application of the Nyquist fidelity assessment method to real world complex systems are shown. These are the Thirty Meter Telescope (TMT) and the Stratospheric Observatory for Infrared Astronomy (SOFIA). Both of these programs are established and integrated models exist for each. The fidelity of these existing models is assessed using the method presented in Chapter 3 and suggestions are made to improve the fidelity of the model.

\subsection{Thirty Meter Telescope}

The Thirty Meter Telescope is a ground-based optical/infrared astronomical research observatory currently in the design development phase. It is scheduled for completion in 2015. It employs adaptive optics to provide diffraction limited performance over 1-2 arc minute fields with sensitivities that will enable imaging of some of the faintest objects in the universe. It will be able to resolve "crowded star fields into individual stars in galaxies as far away as 10 million light years. [9]" The TMT figure shown in Fig. 1-2 is a recent rendering, showing the optical telescope assembly (OTA) enclosed by a large dome.

The model examined in this section is the integrated TMT model developed by MIT in 2004. This model represents an earlier, conceptual design of TMT. A rendering of this earlier design can be seen in Figs. 5-1 (a) and (b). In Fig. 5-1 (a), the telescope structure is shown enclosed inside a dome designed to protect the telescope from stray light, dust, and wind disturbance. Also shown in Fig. 5-1 (a) are the wind disturbance paths through the dome. Fig. 5-1 (b) shows a more detailed view of the telescope structure 
without the dome. The structural supports underneath the primary mirror provide support and rotation about the elevation axis. To the side of the primary mirror, a platform for scientific instruments is visible. The main difference between the conceptual and current designs is that in the previous design the elevation axis is below the primary mirror and in the current design it is above the primary mirror, making the telescope shorter.

The purpose of the integrated model based on this design is to examine the trades between optical performance, mass, and cost over a wide range of design options. Therefore, a parametric model was created to rapidly evaluate different design configurations to reveal trends in optical performance, cost and mass. The tradespace results can be seen in Uebelhart [77] and [79].

Here we examine the model in the nominal configuration and evaluate the fidelity level of the structural model using the steps shown in Section 3.6

\subsubsection{TMT Model and Fidelity}

The TMT integrated model is designed to estimate mass and cost based on key design parameters and to estimate optical performances based on design parameters and disturbances. The integrated model (IM) consists of structural, optical, control and disturbance subsystems. The IM diagram can be seen in Fig. 5-2, The TMT Plant block contains the state space structural model of the OTA. In this model, only the OTA and part of the elevation structure are simulated. The dome, azimuthal structure, and instrument platform are absent since they are not needed for this analysis.

The optical model is represented by linear sensitivity matrices, shown as $C_{1}$ and $C_{2}$ in Fig. 5-2, These matrices transform structural displacements into Zernikes, which are optical metrics. Two controllers, $K_{P M}$ and $K_{S M}$ are the primary and secondary mirror controllers, respectively. They impose forces and torques on the structure according to Zernike values. These controllers are based on the control design shown in MacMartin [52]. The wind disturbance, shown here as an input to the plant, is represented as frequency based disturbance spectra. The outputs of the model are the Zernike values. These are translated into the output figures of merit (OFM) using equations discussed below.

For the fidelity assessment, the structural model of the OTA and part of the elevation structure are examined. The OTA consists of the primary and secondary mirrors, the secondary tower structure (SST), 


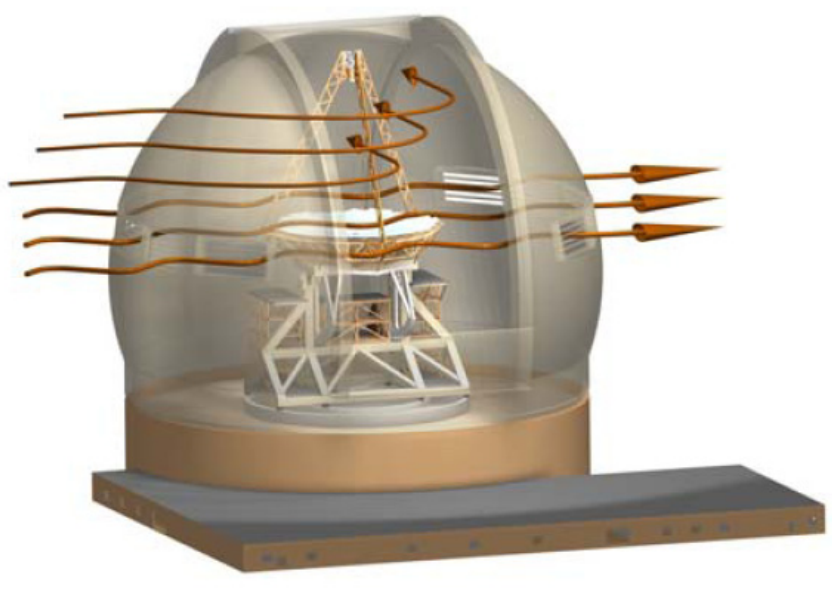

(a) Thirty Meter Telescope [7]

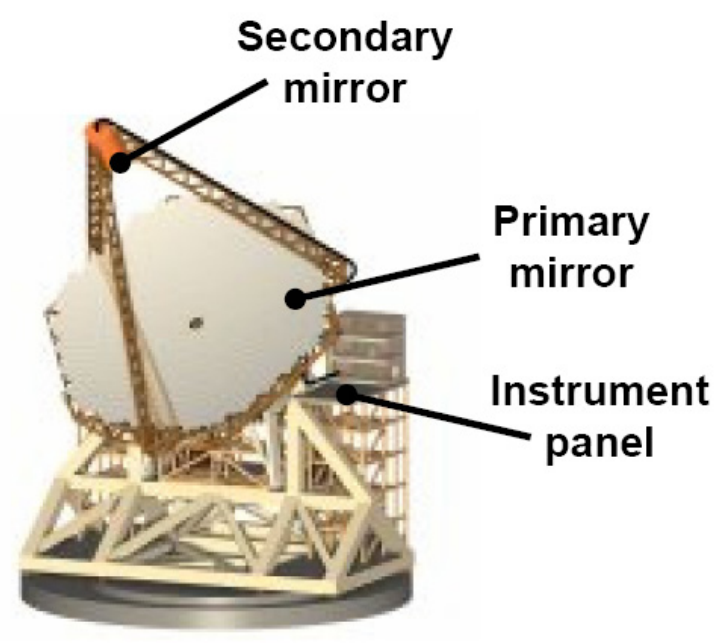

(b) Optical Telescope Assmebly of TMT [9]

Figure 5-1. TMT

and the primary mirror backstructure. The elevation structure consists of a lattice structure and elevation axis connection bars. The FEM of the OTA and elevation structure can be seen in Fig. 5-3.

\section{Step 1: Initialize model}

The first step is to define the model properties, geometry, disturbances and OFM. Like the MOST model, the TMT model is a parametric model where the integrated model is automatically generated based on a set of design parameters. The nominal design parameters and their values are shown in Table 5.1. The elevation axis distance is the vertical distance between the PM surface and the elevation axis points. The wireframe FEM of the TMT structure shows the geometry of the nominal model and can be seen in Fig. 5-3. It is attached to ground via springs attached to the elevation axis connection points. The elevation axis structure is partially modeled as a lattice structure and eight elevation axis support bars. The azimuthal structure is not explicitly modeled in this instance.

The thirty meter diameter primary mirror is made up of approximately 700 hexagonal segments. The segments are broken up into groups of seven segments. Each group is supported by a structure called a raft. Each raft and seven-segment group is modeled as a concentrated mass and is connected to the primary mirror backstructure via a stiff spring and beam elements. The primary mirror backstructure is made up of 


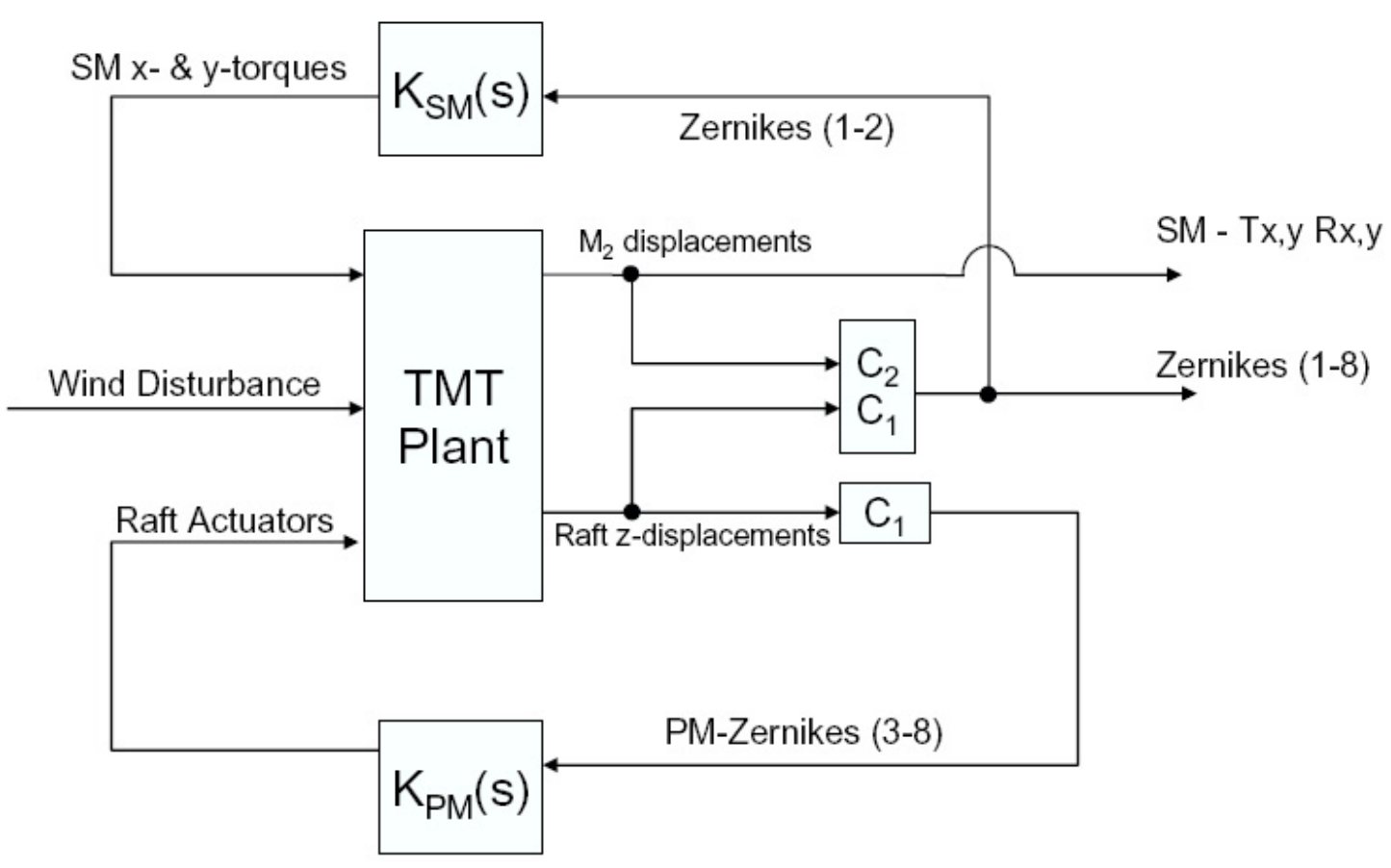

Figure 5-2. Integrated model diagram for TMT

hexapod cells. Each cell supports a raft. Four rafts are shown in Fig. 5-5, each supported by a hexapod cell, shown as a triangle. A side-view schematic of the raft mass connection to the PM backstructure can be seen in Fig. 5-4. The raft mass is connected to the PM backstructure via a zero-length spring and three raft support bars. Each bar connects the spring to the vertices of the hexapod cell. Note that the location of the actuators for primary mirror control loop are at the spring location in Fig. 5-4.

The SST is in a tripod shape and is made up of three two-dimensional trusses. Four cables are attached from two points on each SST truss to the PM backstructure. Attached near the top of the SST is the secondary mirror (SM) which is modeled as a concentrated mass. Similar to the PM raft mounts, the SM is mounted on a spring connected to rigid body elements which connect to the SST. The secondary mirror force actuators are located in this spring.

The entire structure, is comprised of beams, cables, springs, concentrated masses and rigid body elements. The cables' contribution to the fidelity assessment is ignored for this demonstration since the longitudinal deformations have not been treated using Nyquist. Also the springs do not have a direct treatment in the Nyquist fidelity assessment method. The Nyquist fidelity assessment method is used to examine the 
beams and the mirror masses in the structure.

The next step is to examine the disturbances. Since TMT is a ground based observatory, the major disturbance force encountered is from wind. Other disturbances consist of seismic and atmospheric disturbances. Seismic forces either have a very long time scale, on the order of years, or have a very short time scale, corresponding to earthquakes, which primarily are examined for failure conditions. Since this model simulates operating conditions over a relatively short time scale, seismic forces are ignored. While atmospheric disturbances distort the incoming science light, a separate optical performance budget is is allocated to optical degradation due to atmospheric disturbance. Therefore the main disturbance source examined in this model is wind disturbance.

The TMT is enclosed in a dome. Therefore, the wind disturbance on the OTA originates either from the slit (through which science light is observed) or from the vents shown as arrows in Fig. 5-1 (a). The wind disturbance model was built by the NOAO [53]. Four disturbance PSDs are defined: (1) forces on the primary mirror from the slit (M1s), (2) forces on the primary mirror from the vents, (3) forces on the secondary mirror from the slit, and (4) forces on the primary mirror due to the shear layer mode. The shear layer mode is due to vortices passing over the slit and is represented as a force applied at a single frequency with constant amplitude across the primary mirror surface. Wind forces are also imparted onto the SST, however, the mirrors make up a majority of the surface area over which the forces act, so the wind forces on the SST are ignored. The shear layer mode is a tone and therefore contains a single frequency. The three wind PSDs are shown in Fig. 5-6. It can be seen that the upper disturbance frequency in all four cases is 20 $\mathrm{Hz}$. The maximum frequency is a parameter set in the wind model set to $20 \mathrm{~Hz}$ as the nominal value. This is the upper maximum frequency considered for the Nyquist fidelity method. The frequency of the shear layer mode varies with the configuration. The shear layer frequency for the nominal model is $0.48 \mathrm{~Hz}$, well within the $20 \mathrm{~Hz}$ bandwidth.

The last part of Step 1 is to define the OFM. In this case, the OFM are the image motion and image quality, given as

$$
\begin{aligned}
& \text { image motion }=\mathrm{IM}=\sqrt{\sum_{j=1}^{4} \sum_{\mathrm{i}=2}^{3} \sigma_{i, j}^{2}} \\
& \text { image quality }=\mathrm{IM}=\sqrt{\sum_{j=1}^{4} \sum_{\mathrm{i}=4}^{9} \sigma_{i, j}^{2}}
\end{aligned}
$$


Table 5.1. TMT nominal design values

\begin{tabular}{lc}
\hline \hline Design Variable & Value \\
\hline Elevation axis [m] & 3.5 \\
PM focal ratio & 1.0 \\
PM segment radius [m] & 0.5 \\
Final focal ratio & -15 \\
Elevation angle [deg] & 25 \\
Optical control bandwidth [Hz] & 1 \\
\hline
\end{tabular}

where $\sigma_{i, j}$ is the $i^{\text {th }}$ RMS Zernike coefficient resulting from the $j^{\text {th }}$ disturbance source. There are four disturbance sources, listed above. The image motion is similar to line-of-sight jitter and is determined by the second and third Zernikes which represent tip and tilt of the image. The image quality is determined by the higher order Zernikes. The Zernikes, in turn, are determined by mirror displacements from the IM. Mass and financial cost are also determined from this model. Since cost and mass are not displacement-based OFM, they are not subject to the fidelity assessment.

\section{Step 2: Identify important components}

Two networks are shown in Fig. 5-7. The first is the network of the full system with the major subsystems and the connection to the ground shown. Since the nominal configuration is a Nasmyth focus, the scientific instruments are mounted on a platform to the side of the OTA, which is attached to the structure providing the azimuthal degree of freedom. The major subsystem not shown is the dome which is not connected to the azimuthal structure.

The second network, Fig. 5-7(b), represents the network of the TMT model presented in this section. Note that only specific wind disturbances are examined and the atmospheric and seismic disturbances are ignored. Furthermore, the azimuthal structure is not modeled. The elevation structure is partially modeled using the lattice structure and the elevation axis support bars shown in Fig. 5-3. The elevation axis connection points are connected to ground via stiff springs attached to these points. The tertiary mirror and scientific instruments are also not included in the model.

By examining the second network, the important components can be identified. The important subsystems are the OTA components since they occupy the path from the disturbance origin to the structures that determine the OFM values. The OTA consists of the mirrors, the SST and the PM backstructure. The 


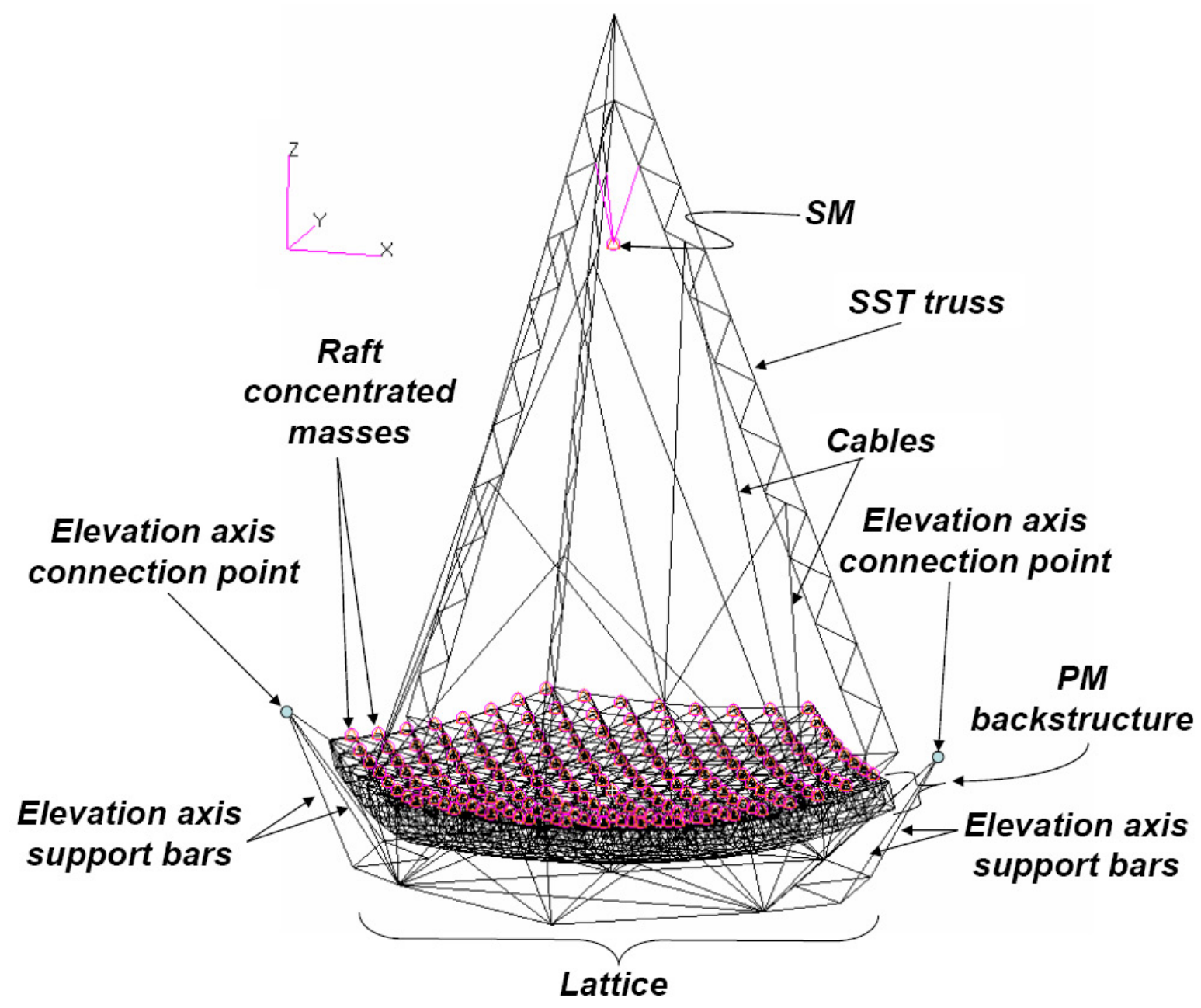

Figure 5-3. TMT structural FEM

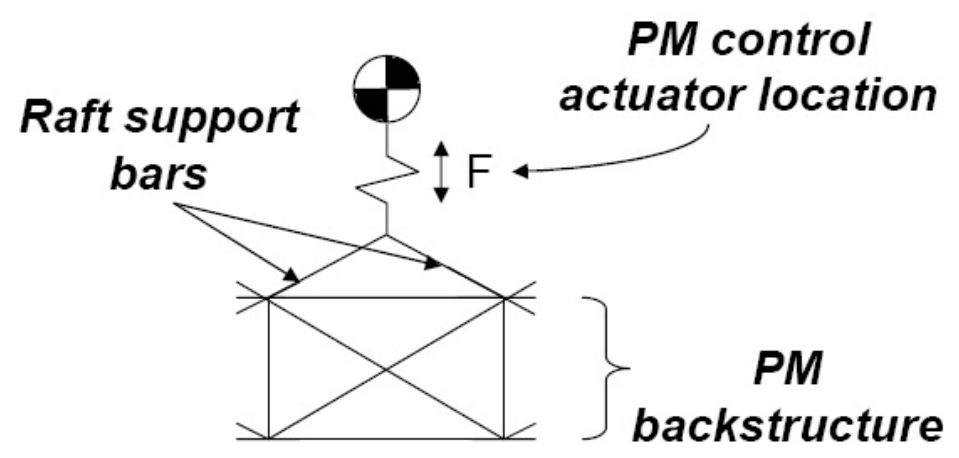

Figure 5-4. Raft support schematic [78] 


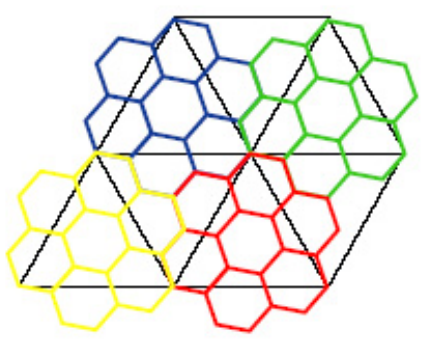

Figure 5-5. TMT raft layout [78]

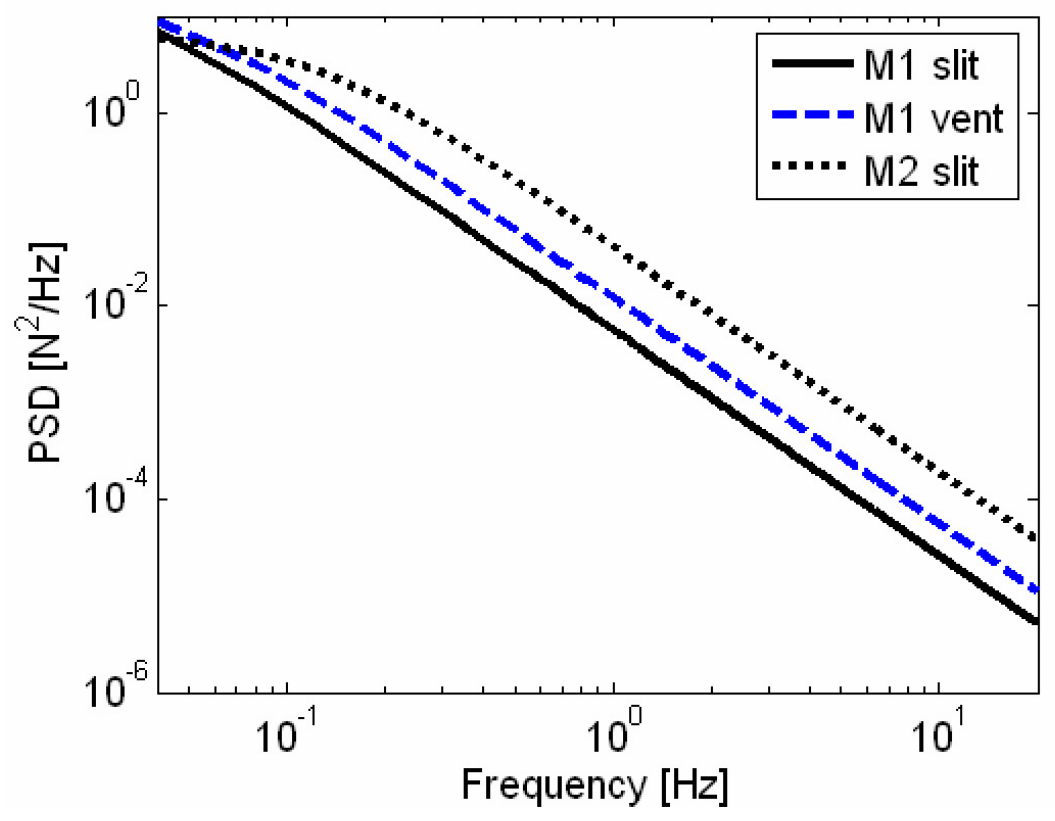

Figure 5-6. Wind disturbance PSDs 
structures that determine the OFM values are the mirrors. The elevation structure, while not lying on the disturbance path, cannot be considered an appendage since it provides support to the important subsystems and contains the boundary condition to ground. Therefore, the elevation structure is also considered an important component in the fidelity analysis.

In the model shown in Fig. 5-3, each beam is made up of one element. Each beam is examined and assessed for fidelity in the next section. In addition, the primary and secondary mirrors are examined to determine if it is appropriate to model them as concentrated masses.

\section{Step 3: Calculate fidelity}

The model shown in Fig. 5-3 contains 2335 beams. They can be separated into five groups representing five different beam properties: the PM backstructure, the raft support bars, the SST, the bars connected to the elevation axis points, and the lattice structure. Using fidelity methods and the upper disturbance frequency of $20 \mathrm{~Hz}$, the fidelity of each beam can be calculated. These results are shown as histograms in Figs. 5-8 and 5-9. Recall that there are only eight bars connecting the elevation axis to the lattice structure, four bars connecting to each point. There are many smaller bars in the PM backstructure and raft support bars, all with fidelity levels between 4.0 and 5.0. There are two bars that do not meet the minimum fidelity requirement of 1.0, shown in Fig. 5-9. They are lattice bars along the bottom of the lattice. All the other bars have fidelities between 1 and 5. The lattice and the elevation axis support bars are shown in Fig. 5-10 and the bars with fidelities less than one are highlighted in the isometric view using dashed lines. The bars in question lie directly below these arrows. These two bars are the longest bars in the lattice at $22.5 \mathrm{~m}$ and are modeled with a single beam element, which does not meet fidelity requirements.

The mirrors can also be examined. Here, they are modeled as concentrated elements and are assumed to be made of Schott-Zerodur ${ }^{\circledR}$ glass. Zerodur is advantageous since it has a very low thermal expansion coefficient. The first bending frequencies of the primary mirror segments and secondary monolithic mirror are estimated as $66.2 \mathrm{~Hz}$ and $36.3 \mathrm{~Hz}$, respectively using the Leissa reference [48]. The first bending frequencies exceed the upper disturbance frequency of $20 \mathrm{~Hz}$ and therefore the excitation of due to the wind does not produce bending behavior in these plates. Therefore, the dynamic bending behavior of the plates does not contribute to the OFM and it is valid to model the mirrors as concentrated masses. The 


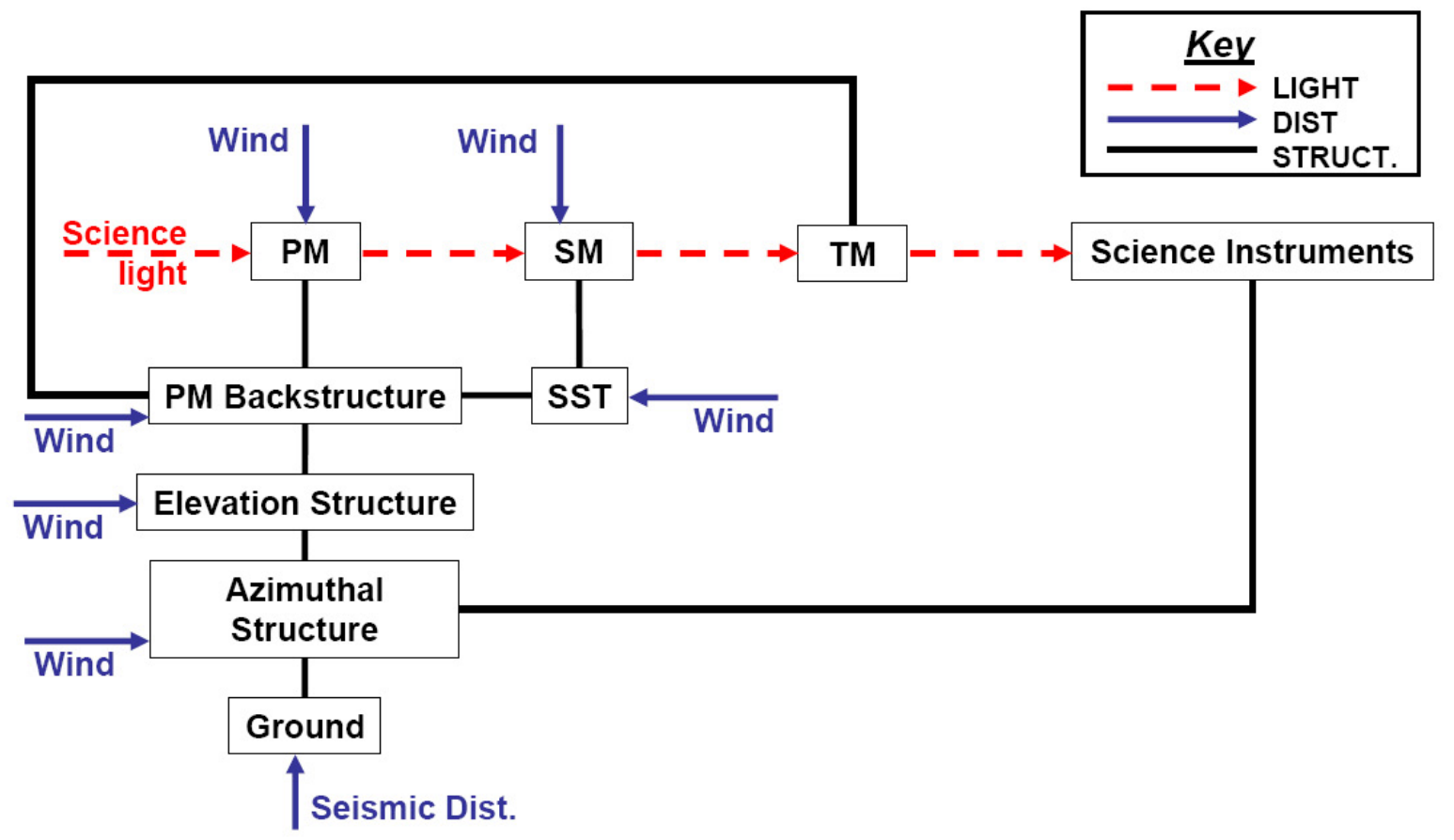

(a) Full TMT network [7]
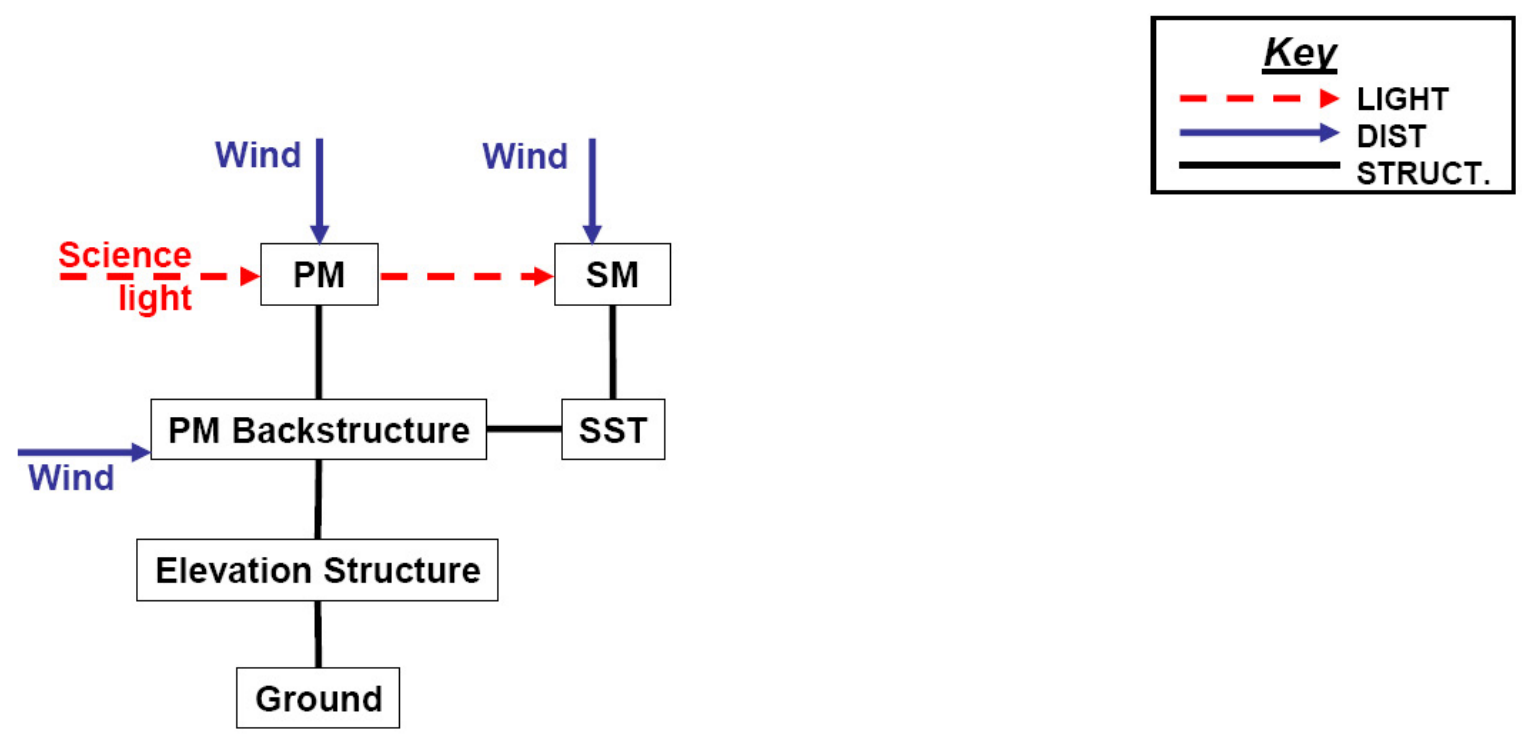

(b) TMT network for model presented

Figure 5-7. TMT networks 


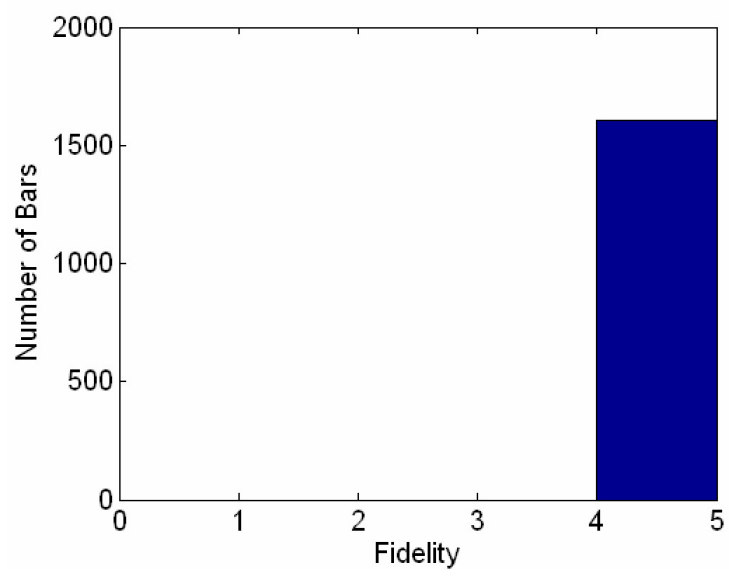

(a) PM backstructure

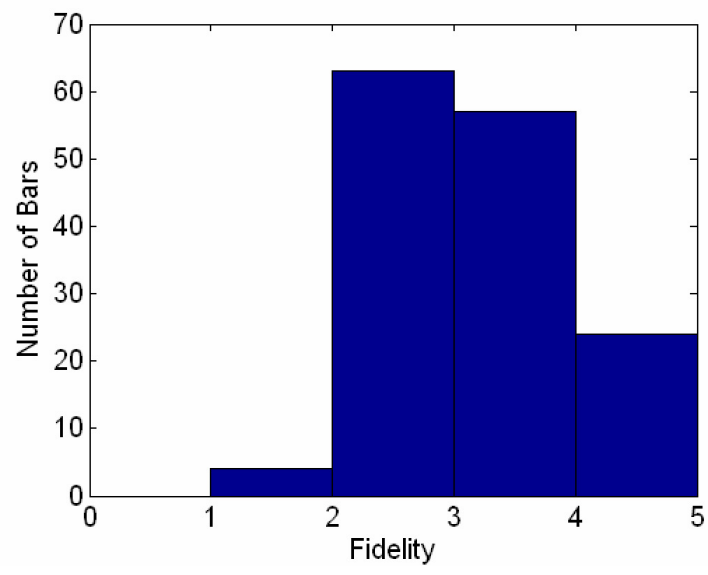

(c) SST

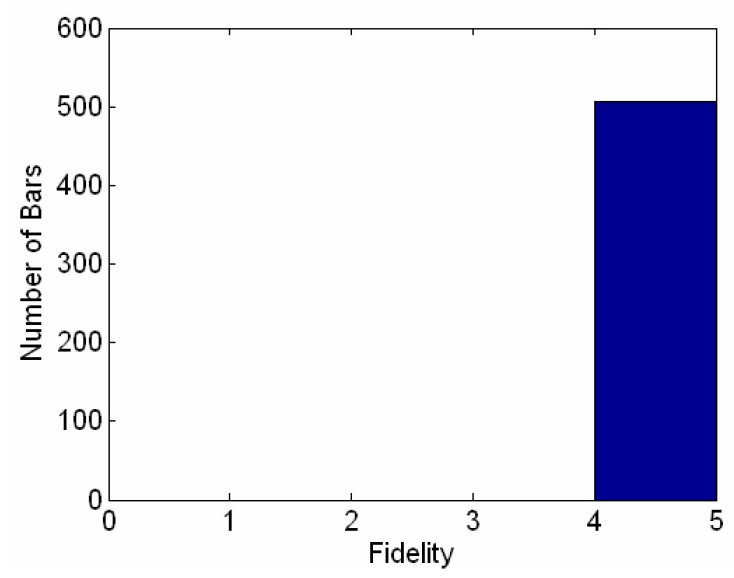

(b) Raft supports

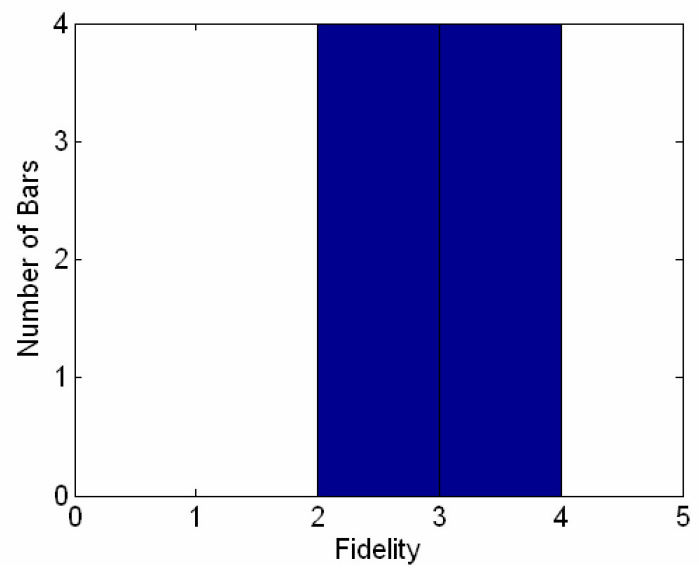

(d) Elevation axis

Figure 5-8. Quantity of bars at each fidelity level

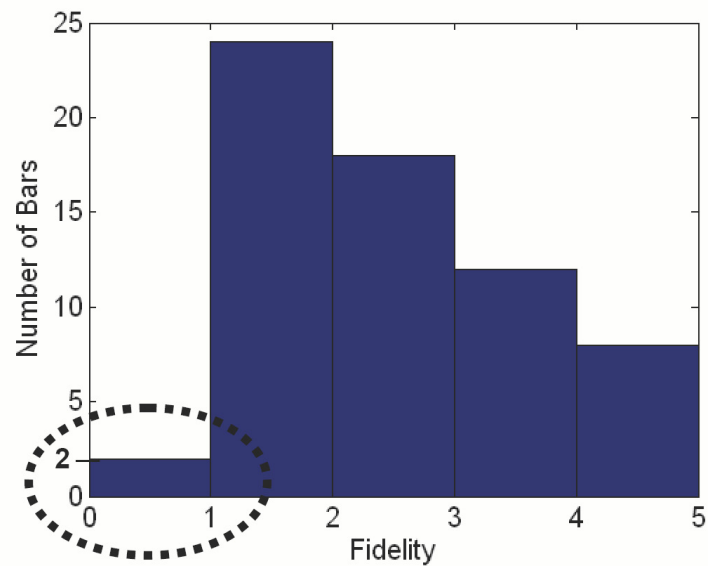

Figure 5-9. Quantity of lattice bars at each fidelity level 


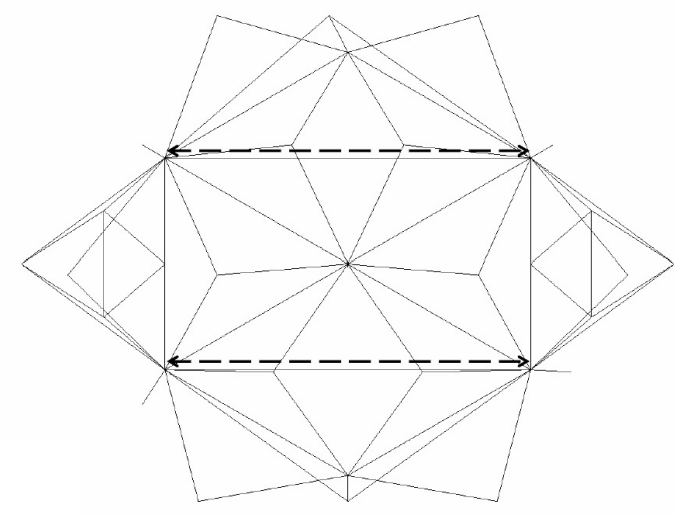

TOP

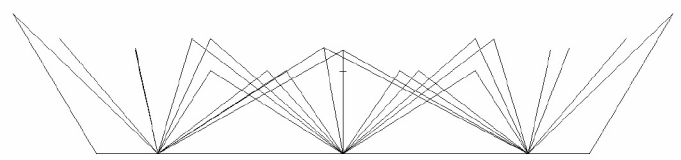

FRONT

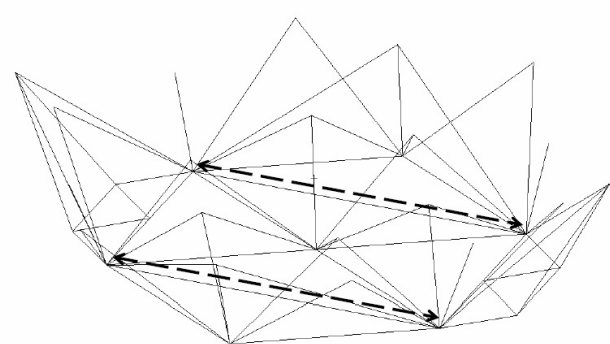

ISOMETRIC

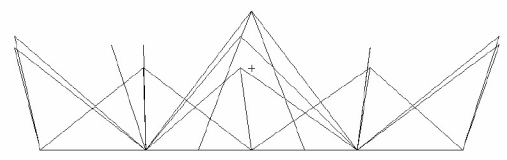

SIDE

Figure 5-10. Four views of the lattice and elevation axis bars

concentrated mass provides the representative inertia and the springs supporting the masses represent the equivalent quasi-static compliance of a plate.

\section{Step 4: Estimate modal frequency error}

Once the fidelities of each beam are calculated, the modal frequency error is estimated using Eqn. 3.12 . Figure 5-11 shows the modal frequency errors for the beams plotted against fidelity divided into the five groups of bars defined in Step 3. and broken up according to the five groups. The data point in Fig. 5-11 to the left of the minimum fidelity requirement is an overlap of the data points corresponding to the two lattice bars that have fidelity levels less than 1.0.

Besides the two offending lattice bars, the next lowest fidelity level across all the bars is 1.30 , present in the lattice and SST truss members. This corresponds to a modal frequency error of $2.08 \%$. Therefore, if the two offending lattice bars where sufficiently meshed to have a fidelity greater than or equal to 1.30 , then the expected modal frequency error in the model due to under-meshing would be $2.08 \%$. This is used in the next section to estimate OFM error. 


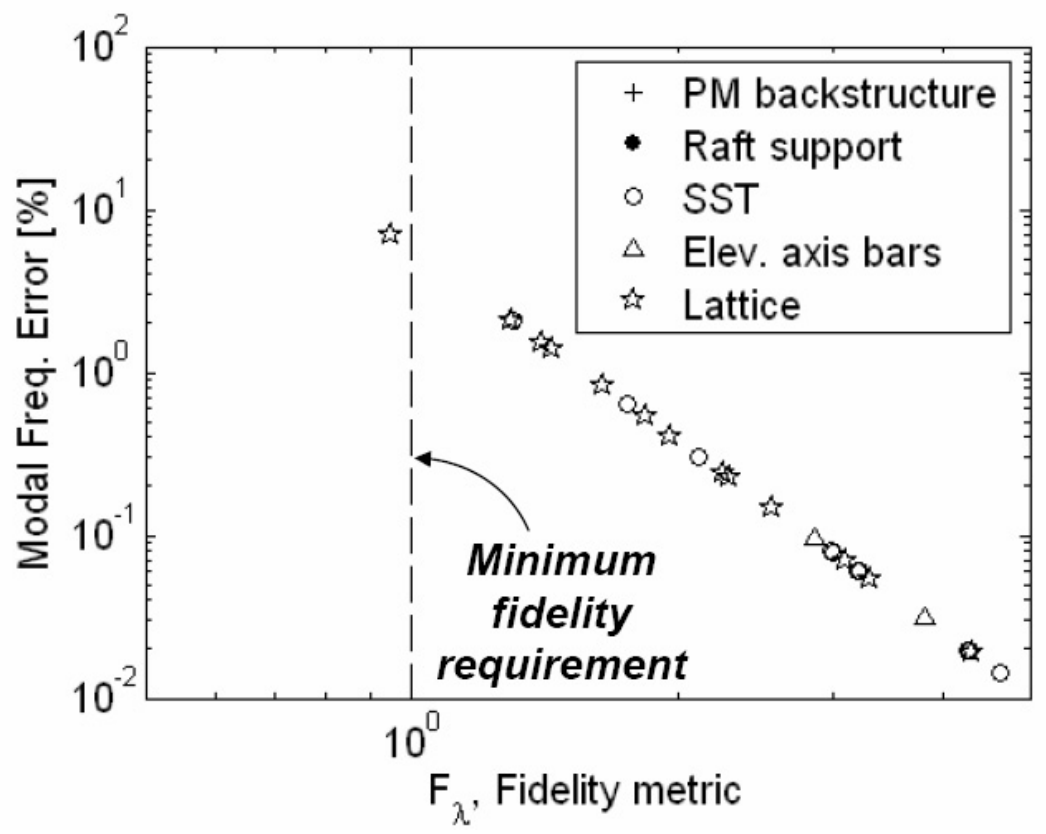

Figure 5-11. MFE vs. fidelity for TMT bars

\subsubsection{TMT error estimates}

To estimate the errors in the image motion and image quality, first the nominal performances are evaluated. These are

$$
\begin{aligned}
& \mathrm{IM}=2.6126 \mathrm{E}-5[\mathrm{~m}] \\
& \mathrm{IQ}=6.3102 \mathrm{E}-7[\mathrm{~m}]
\end{aligned}
$$

This is based on 100 modes extracted from the FEM. In fact, the highest mode extracted is at $17.08 \mathrm{~Hz}$, which falls below the upper disturbance frequency of $20 \mathrm{~Hz}$. Adjusting the analysis such that all modes at or below $20 \mathrm{~Hz}$ are extracted (total of 237 modes), gives slightly lower nominal performances of

$$
\begin{aligned}
& \mathrm{IM}=2.6124 \mathrm{E}-5[\mathrm{~m}] \\
& \mathrm{IQ}=6.2901 \mathrm{E}-7[\mathrm{~m}]
\end{aligned}
$$

Next, a sensitivity analysis is performed, which yields the sensitivity of the Zernike RMS values with respect to each system mode for each disturbance source. These sensitivities are labeled as:

$$
\frac{\partial \sigma_{M 1 s_{i}}}{\partial \omega_{k}}, \frac{\partial \sigma_{M 1 v_{i}}}{\partial \omega_{k}}, \frac{\partial \sigma_{M 2 s_{i}}}{\partial \omega_{k}}, \frac{\partial \sigma_{S H_{i}}}{\partial \omega_{k}}
$$


where $\sigma$ represents the Zernike RMS value, and $\omega_{k}$ is the modal frequency of the $k^{\text {th }}$ structural mode. Note that since controllers are present in the integrated model, the system modes include controller modes and therefore do not correspond one-to-one with the structural modes. The subscripts $M 1 s, M 1 v, M 2 s$, and $S H$ represent the disturbance forces on the primary mirror due to the wind entering through the slit, wind disturbing the primary mirror through the vents, wind disturbing the secondary mirror through the slit, and the shear layer mode, respectively. The subscript $i$ indicates the Zernike number. The reason that the sensitivity analyses are broken up according to disturbance sources is because a single sensitivity analysis incorporating all the disturbance sources is prohibitively expensive. However, the method employed here does not alter the error estimation procedure since the disturbances are assumed independent (uncorrelated).

The sensitivity of the OFM with respect to the frequencies of the structural modes is calculated using the chain rule on Eqn. 5.1. These sensitivities are:

$$
\begin{aligned}
& \frac{\partial I M}{\partial \omega_{k}}=\left[\sum_{j=1}^{4} \sum_{i=2}^{3} \sigma_{i, j}^{2}\right]^{-1 / 2}\left[\sum_{j=1}^{4} \sum_{i=2}^{3} \sigma_{i, j} \frac{\partial \sigma_{i, j}}{\partial \omega_{k}}\right] \\
& \frac{\partial I Q}{\partial \omega_{k}}=\left[\sum_{j=1}^{4} \sum_{i=4}^{9} \sigma_{i, j}^{2}\right]^{-1 / 2}\left[\sum_{j=1}^{4} \sum_{i=4}^{9} \sigma_{i, j} \frac{\partial \sigma_{i, j}}{\partial \omega_{k}}\right]
\end{aligned}
$$

The index $i$ indicates Zernike number. The index $j$ is the index to the four disturbance sources. The variable $\sigma_{i, j}$ is the nominal RMS value of the $i^{\text {th }}$ Zernike coefficient due to the $j^{\text {th }}$ disturbance force. The difference between the two expressions in Eqn. 5.3 are the $j$ indices. The OFM error estimate bounds are then calculated using equations similar to 3.28 and 3.29 . Assuming that

$$
\begin{aligned}
& \frac{\partial I M}{\partial \omega_{k}} \approx \frac{\Delta I M}{\Delta \omega_{k}} \\
& \frac{\partial I Q}{\partial \omega_{k}} \approx \frac{\Delta I Q}{\Delta \omega_{k}}
\end{aligned}
$$

the percent change in the OFM with respect to the percent change in the structural modal frequencies can be expressed as

$$
\begin{aligned}
& \frac{\text { \%changein } I M}{\text { \%changein } \omega_{k}} \approx \frac{\partial I M_{/} / M_{\text {nom }}}{\partial \omega_{k} / \omega_{k, n o m}}=\frac{\partial I M}{\partial \omega_{k}} \frac{\omega_{k, \text { nom }}}{I M_{\text {nom }}} \\
& \frac{\partial I Q_{/} / Q_{\text {nom }}}{\text { \%changein } I Q}=\frac{\partial I Q}{\partial \omega_{k}} \frac{\omega_{k, \text { nom }}}{I Q_{\text {nom }}}
\end{aligned}
$$


where $\omega_{k}$ is the $k^{t h}$ structural mode. The OFM error bounds are estimated as

$$
\begin{aligned}
& \delta I M=\sum_{k=1}^{m}\left|\frac{\% \text { changein } I M}{\% \text { changein } \omega_{k}}\right|(\delta \omega) \\
& \delta I Q=\sum_{k=1}^{m}\left|\frac{\% \text { changein } I Q}{\% \text { changein } \omega_{k}}\right|(\delta \omega)
\end{aligned}
$$

where $m$ is the number of structural modes below the disturbance frequency and $\delta \omega$ is the calculated modal frequency error.

With a $2.08 \%$ MFE, the OFM error estimate bounds are:

$$
\begin{aligned}
& \delta_{I M}=-473.45 \% \\
& \delta_{I Q}=-402.99 \%
\end{aligned}
$$

These are extremely large numbers are due to the high sensitivity of these OFM to the structural modes. Recall that this error measure does not indicate how much error is present in the OFM, rather it is an estimate of the error bound on the OFM due to under-meshing. Therefore the actual error in the OFM due to under-meshing is equal to or below these values.

As a comparison, were all the beams meshed sufficiently fine to produce a $0.01 \%$ modal frequency error, the OFM error estimates would be

$$
\begin{aligned}
& \delta_{I M}=2.2762 \% \\
& \delta_{I Q}=1.9374 \%
\end{aligned}
$$

which is a significant improvement in the error bound estimate. In order to achieve this, however, every beam in the model would have to be re-meshed, however, there would be significantly more confidence in the model since the error bound estimates are so dramatically reduced.

\subsubsection{TMT summary}

The fidelity assessment of the nominal TMT structural model is presented in this section. The structural model is made up of beams, cables, concentrated masses, springs, and rigid body elements. The fidelity of the beams and concentrated masses is assessed. In the existing TMT nominal model, the FE mesh size decision was based on engineering judgement.

Here, Nyquist fidelity analysis reveals two beams in the lattice structure do not contain sufficient fidelity 
to represent modeshapes within the disturbance bandwidth of $20 \mathrm{~Hz}$. The remainder of the beams in the model meet the minimum fidelity requirement of 1.0. This minimum fidelity requirement was established in Section 3.3. The modal frequencies of the primary mirror segments and the secondary mirror plates are estimated to ensure that their modal frequencies lie above the upper disturbance frequency. It was determined that it is acceptable to model the mirrors as they were as concentrated masses mounted on springs. For demonstration purposes, the two lattice beams are ignored and the modal frequency error of the system is estimated at $2.08 \%$. This indicates a bound on the modal frequency error in the structural modes. Sensitivity analysis is then used to translate the modal frequency error into error bounds on the output figures of merit, image motion and image quality. These error bounds were above $400 \%$. It was shown that with a modal frequency error of only $0.01 \%$, an OFM error bound of $2 \%$ could be achieved. This would require finer meshing of all of the beams in the model. 


\subsection{SOFIA}

The Stratospheric Observatory for Infrared Astronomy (SOFIA) is an airborne telescope developed as a cooperative effort between NASA and the DLR Deutsches Zentrum für Luft- und Raumfahrt (German Aerospace Agency) and supported by the Universities Space Research Association (USRA) and the Deutsches SOFIA Institut (DSI). SOFIA has a parabolic $2.7 \mathrm{~m}$ diameter primary mirror and is capable of gathering science light from the visible to the submillimeter (far-infrared) range $(0.3 \mu \mathrm{m}-1600 \mu \mathrm{m})$ with pointing accuracies less than 1 arc second. SOFIA is in the final stages of assembly and test and in April 2007, SOFIA completed its first test flight. SOFIA is scheduled to begin scientific observations in 2010 and is expected to continue observations for 20 years.

Designed to complement the science data gathered by current and future ground and space-based observatories such as Keck, Hubble and the James Webb Space Telescope, SOFIA presents important benefits over ground based and spacecraft telescopes. SOFIA is housed in the aft section of a Boeing 747SP and will perform science observations while flying at 39,000 to 45,000 feet. Therefore it bypasses much of the atmospheric disturbance effects (99\% of the water vapor) encountered by ground telescopes. This allows far-infrared observations, not normally accessible by ground telescopes and dramatically increases the imaging quality over ground based telescopes. The science instruments on SOFIA can also be swapped out while the plane is on the ground, so that state of the art instruments can be used. as more sophisticated instruments are developed, Similarly, if there is a problem with SOFIA, the plane can land for servicing. While the benefit of timely servicing is also present in ground observatories, it is more problematic on spaceborne telescopes. For earth orbiting space telescopes, servicing can be accomplished using costly human spaceflight options, such as the Hubble servicing missions. For telescopes operating further afield, the science instruments launched with the telescope are the ones it is designed to use throughout the rest of its life cycle. SOFIA, therefore, supports an important gap in the current astronomical capabilities, since it is both accessible and above most of the atmosphere.

Even though SOFIA is nearly complete and no longer in the conceptual design phase, the fidelity assessment can still be performed on the models used in the design phase. The fidelity of a SOFIA model is assessed in this section. First, a description of the physical system is given, then the fidelity assessment is performed. 


\subsubsection{SOFIA structural description}

A schematic of the SOFIA telescope structural system can be seen in Fig. 5-12, Source: [44]. The SOFIA telescope is in a dumbell configuration which straddles the pressurized and open sections of the fuselage. On one end of the dumbell is the optical telescope assembly (OTA) containing the optical surfaces and the supporting structures. On the other end is the scientific instrument flange (SIF) which includes the science instruments, counterweight systems and gyroscopes.

On the OTA end, the optical surfaces are the $2.7 \mathrm{~m}$ parabolic primary mirror, the $0.35 \mathrm{~m}$ secondary mirror and the small, flat tertiary mirrors. The OTA is in a Cassegrain configuration with a Nasmyth focus, which focuses the light on to the science instruments at the other end of the dumbell. A detailed diagram of the science light path can be seen in Fig. 5-13, Source: [45]. The primary, secondary and tertiary mirrors are labeled M1, M2 and M3, respectively. The tertiary mirror is a dicrohic. Its first surface, M3-1, reflects the visible light to the science instruments and its second surface, M3-2 directs visible light onto a focal plane imager that assists in the pointing. The infrared science light is captured by the cameras on the science instrument flange. Bittner et al. [13] describes the optical system in detail. This end of the dumbell is in the cavity opened to the outside during observations. This cavity is closed during take off and landing using a specially constructed fuselage door.

The components on the SIF end are the contained in the pressurized fuselage portion. The science instruments are designed to be swapped out, and in the model, these are modeled as heavy boxes. The focal plane imager and gyroscopes are also on this end of the dumbell. They assist in dynamic pointing corrections. The couterweight system is used to balance SOFIA since SOFIA is supported in the center of the dumbell configuration using a spherical bearing.

The center of the dumbell consists of the vibration isolation system (VIS), the drive system, the spherical bearing, and the Nasmyth tube. The VIS provides the connection between the aircraft fuselage and SOFIA. It is made up of 12 sets of springs and dampers and is designed to damp out the aircraft vibrations. The drive system, consisting of the coarse and fine drive systems, is located between the VIS and operates on the spherical bearing. The coarse drive system is made up of a roller bearing and geared drive units and is designed to adjust the telescope's gross elevation angle between 16.5 and 68.5 degrees. The fine drive system is powered by a magnetic torquing system acts directly on the spherical bearing. The magnetic 


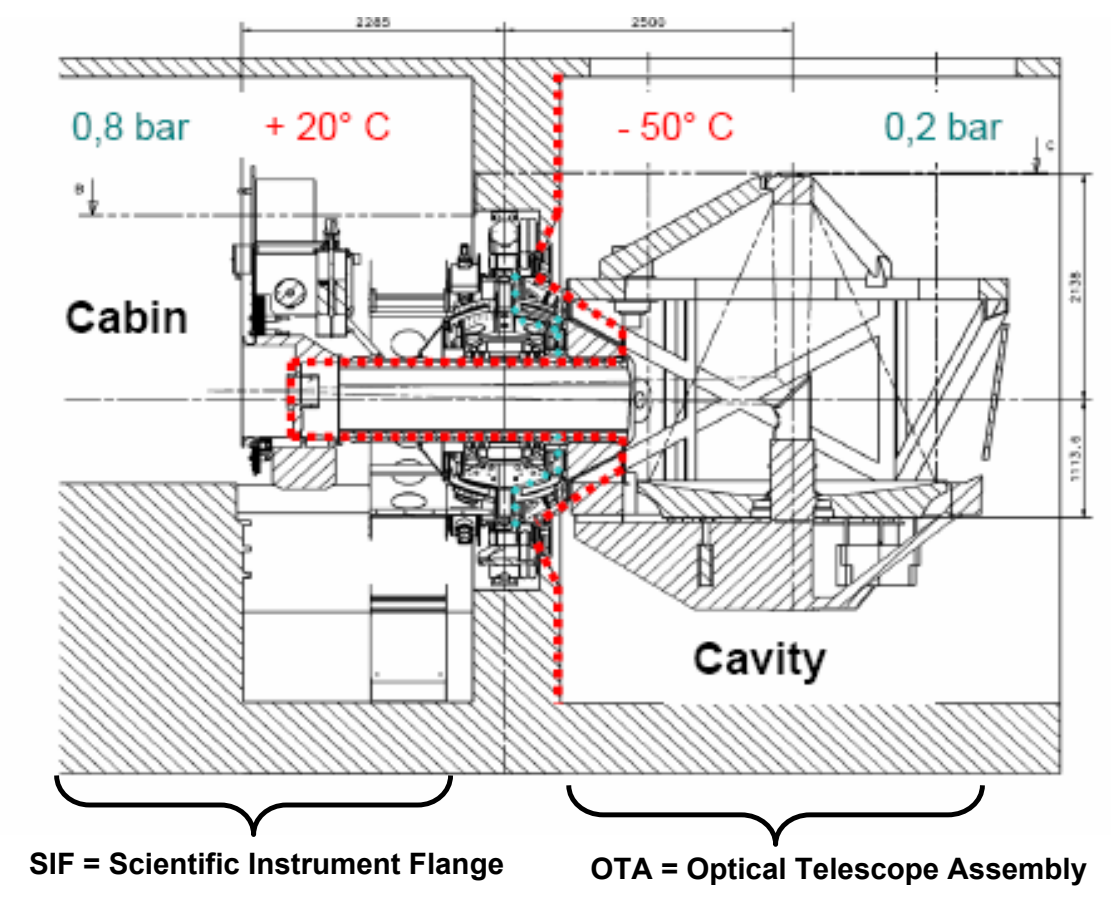

Figure 5-12. Schematic of SOFIA. Source: Kärcher [44]

torquers are used for the fine-tuning of the balance system, the elevation, the cross-elevation and the line-ofsight positions and can adjust the rotational degrees of freedom of the OTA by \pm 3 degrees. Attached to the spherical bearing is the backbone of the structure, the Nasmyth tube. It is through this tube that the science light travels.

The main challenges for SOFIA include counteracting the wind disturbance entering from the fuselage door and the aircraft disturbance entering through the bearing support system. The vibration isolation system consisting of the springs and dampers, an active secondary mirror and the magnetic torquers are used to counteract these dynamic disturbance effects. The Kuiper observatory, a NASA airborne telescope mission operating between 1974 and 1996 encountered the same type of disturbances, but on a much smaller scale; Kuiper had a 36 inch primary mirror compared to SOFIA's 98.6 inch primary mirror.

In the section presented next, the fidelity of SOFIA's structural model is examined. 


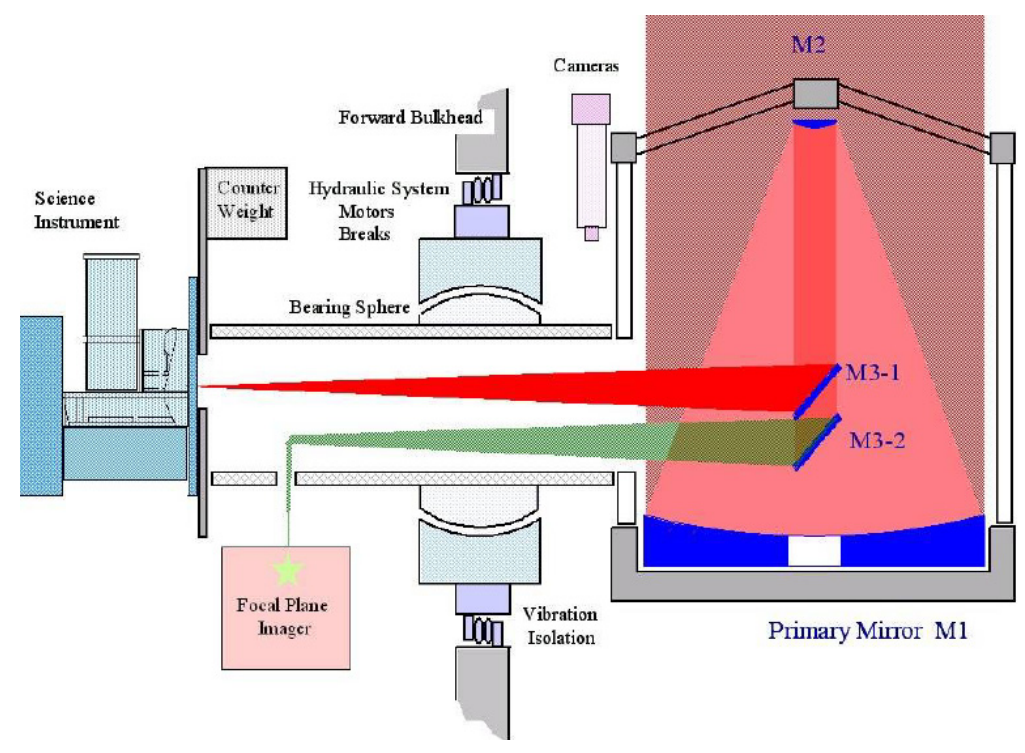

Figure 5-13. Schematic of SOFIA. Source: Krabbe and Kärcher [45]

\subsubsection{Fidelity assessment}

\section{Step 1: Initialize model}

The first step is to define the model properties, geometry, disturbances and output figures of merit (OFM). The overall geometric layout of the SOFIA structural model can be seen in the wireframe finite element model (FEMs) shown in Fig. 5-17. This model was first developed by the telescope's manufacturer, MAN AG of Mainz Germany, then converted to Nastran by Orbital Sciences Corporation [55]. It contains over 70,000 elements and over 331,000 degrees of freedom. The model properties for each of these elements are contained within the finite element model.

The two disturbances, aerodynamic and aircraft, are given as time-domain disturbances. Since the fidelity assessment is based on frequency domain techniques, these disturbances must first be converted to frequency domain power spectral density functions (PSD). These two PSDs are shown in Fig. 5-14. Note that the aircraft disturbance is measured in acceleration units and the aerodynamic disturbance is a force. Both disturbances are sampled at the same rate, $1219.5 \mathrm{~Hz}$, and therefore according to Nyquist, the spectra are reliable up to $610 \mathrm{~Hz}$. This, however, is not the upper disturbance frequency used in the Nyquist fidelity 


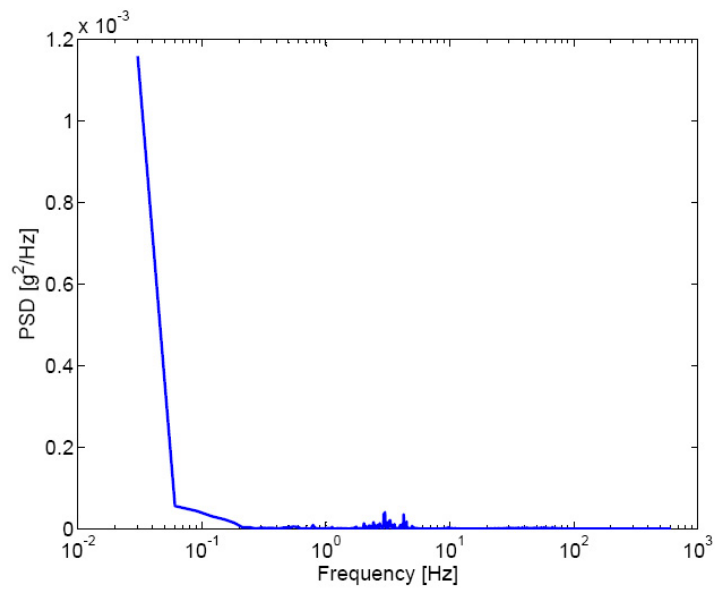

(a) Aircraft disturbance PSD

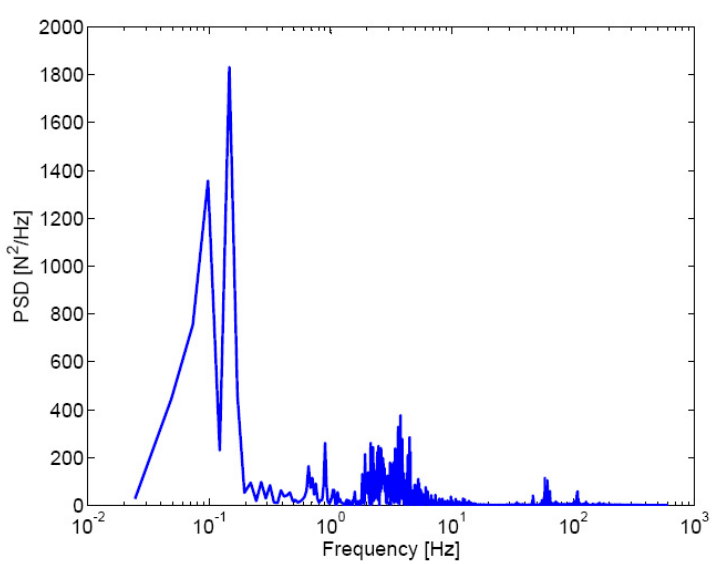

(b) Aerodynamic disturbance PSD

Figure 5-14. Disturbance PSDs for SOFIA

method. The upper disturbance frequency must be chosen. The cumulative RMS plots, which show the running integral of the PSDs in Fig. 5-14 help to determine this; they are shown in Fig. 5-15. Here, the upper frequency for the aircraft disturbance is chosen as $100 \mathrm{~Hz}$, since the cumulative RMS plot is relatively flat above $100 \mathrm{~Hz}$. The aerodynamic disturbance, also, starts a significant, though not complete, leveling-off at about about $100 \mathrm{~Hz}$. Therefore, the upper disturbance frequency chosen here is $100 \mathrm{~Hz}$.

The output figure of merit of this model is the RMS image motion, the output of the integrated model (IM). The SOFIA integrated model (IM) was developed by Ulrich Schönhoff at Darmstadt University of Technology and Martin Süess at MAN AG and simulates the structural, control, optical and disturbance subsystems [74], [75]. It was then further modified by Franziska Harms at the University of Stuttgart [40]. The IM is a state space system representing SOFIA's structural, optical, disturbance, and control dynamics which estimates the image motion due to aerodynamic and aircraft disturbances. The details of the control system can be found in Wandner [81] and Süss et al. [75].

\section{Step 2: Identify important components}

Similar to the MOST and TMT fidelity assessments, a network diagram is constructed which shows the structural connections between subsystems, shown in Fig. 5-16.

The major supporting structures through which the disturbance effects flow through the system are the VIS, the drive system, the spherical bearing, the Nasmyth tube and metering structure. The primary and 


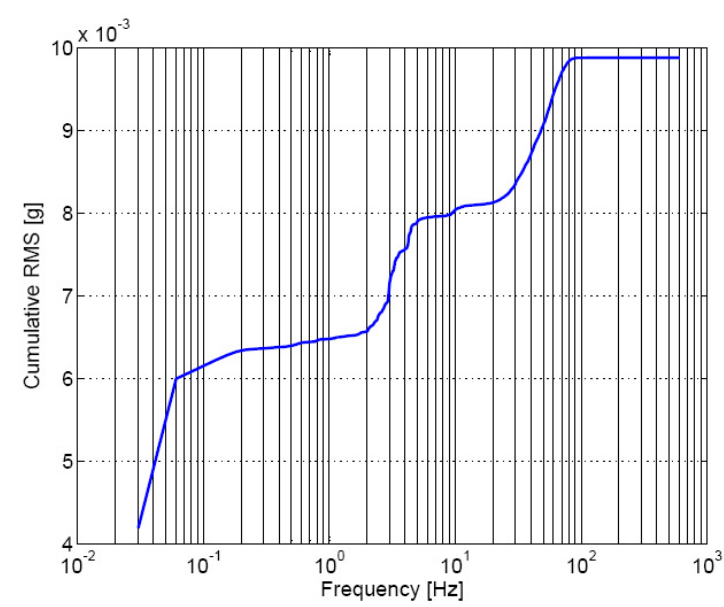

(a) Aircraft disturbance PSD

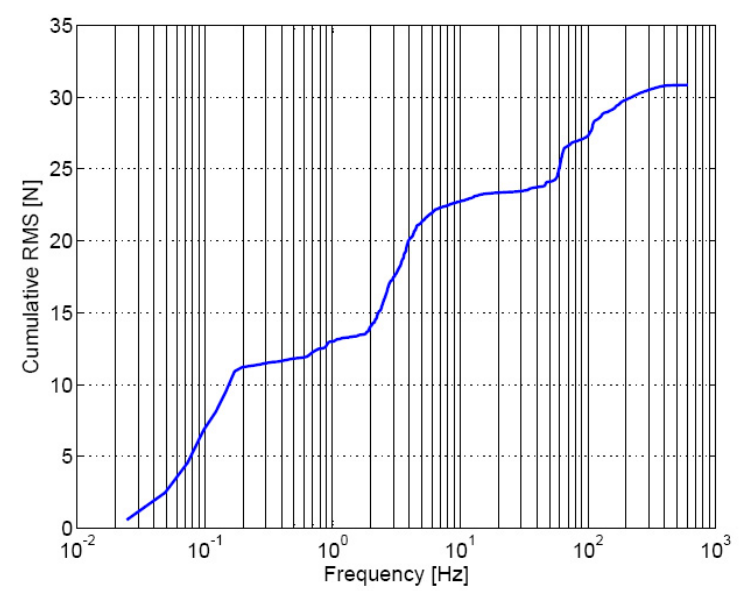

(b) Aerodynamic disturbance PSD

Figure 5-15. Cumulative integrals of SOFIA disturbance PSDs

secondary mirrors also experience disturbance directly through wind forces. The tertiary mirror and the science instrument flange, although they appear to be structural appendages, are also important structures since they directly manipulate the science light. Therefore, as in the TMT case, all the major structures represented in this model are considered important subsystems.

\section{Step 3: Calculate fidelity}

The FEM shown in Fig. 5-17 is made up of beams, plates, solid elements, springs, dampers, concentrated masses, and rigid body elements. The beam and plates in each of the systems shown in Fig. 5-16 are examined here. Since the SOFIA model contains a large number of beam and plate elements (74994) and since the SOFIA topology is so varied, the fidelity of each individual beam and plate element is examined. This is done because the fidelity can be assessed quickly for individual elements using programming code. Examining each large plate or beam would require much more time to manually separate the system into plates. This method is equivalent to examining the fidelity of a larger beam or plate meshed into smaller, individual beam or plate elements and provides the same fidelity assessment values, but just for a larger number of elements.

First, the plates are examined. Each of the important subsystems except for the secondary mirror (SM) and tertiary mirror (TM) contain plate elements. The SM and TM are modeled as a concentrated masses. 


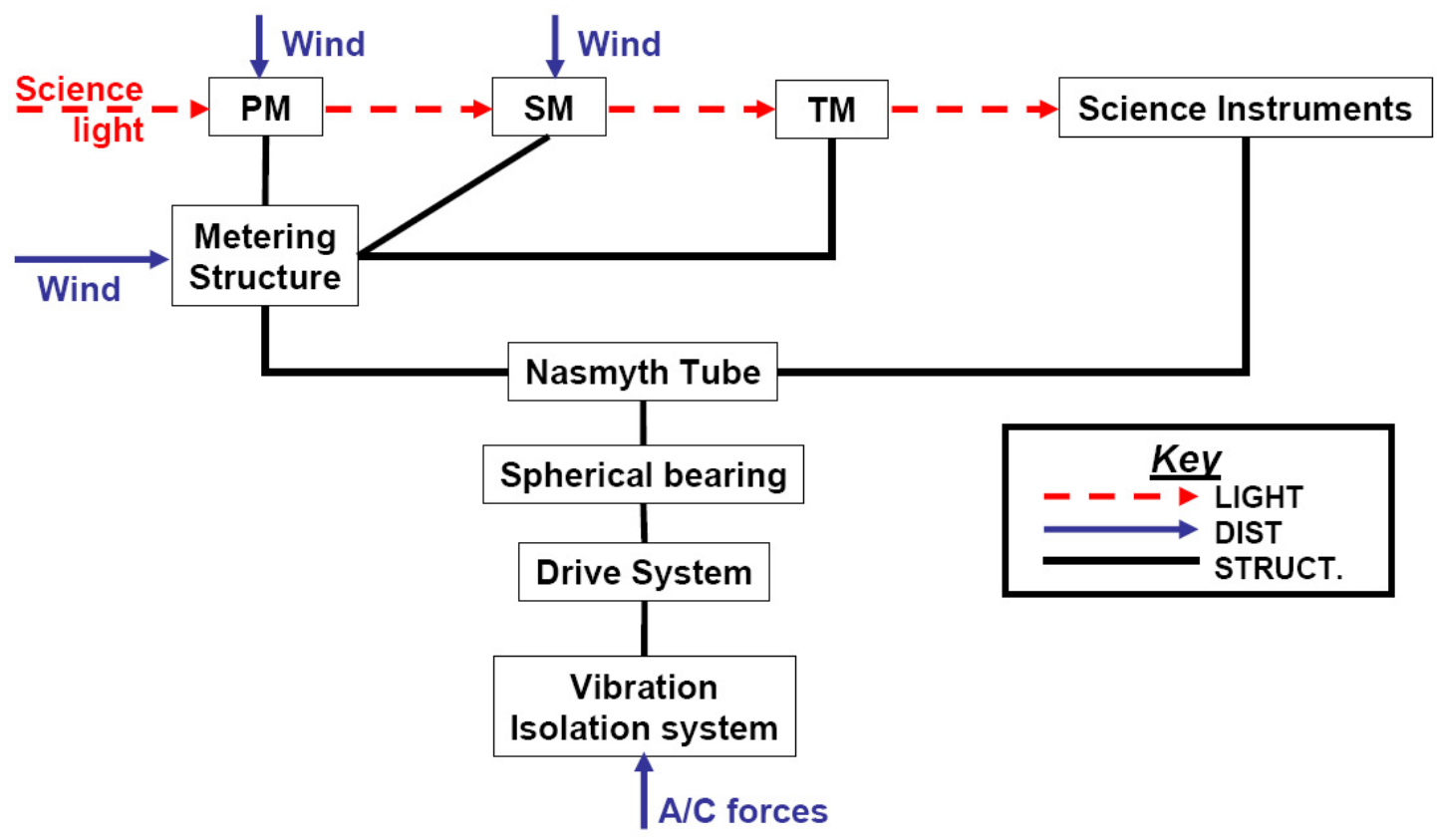

Figure 5-16. Network of SOFIA's structural model

Figures 5-18 and 5-19 show seven histograms displaying the quantity of plates at each fidelity level in each subsystem. Only fidelity levels up to 20 are shown for comparison purposes. Some of these subsystems contain elements with fidelities greater than 20. The VIS, the drive system, and the science instrument flange have plate elements with fidelities below the recommended minimum level of four. The rest of the subsystems meet or exceed this requirement. In the VIS system, there is a total of 7297 plates and 1461 plates have fidelity levels less than four. They correspond to the VIS plates arranged as spokes and some of the side plates. The spoke and the side plates are shown alongside the entire VIS system in Fig. 5-20. In the drive system, the 288 low fidelity plates are the inner and outer plates that represent the magnetic torquers. The magnetic torquer plates and the entire drive system is shown in Fig 5-21. There are only eight plates in the science instrument flange with fidelity levels less than four, between 3.75 and 3.83. These occur on the underside of the flange. These plates require only minimal refinement in order to meet the recommended minimum level of four. It is suggested that these plates be refined further. Some of the plates are over-meshed while others are under-meshed. The Nyquist fidelity method leads to more uniform fidelity throughout the system model.

Next, the beams are examined. The vibration isolation system, the metering structure, and the science 
instrument flange contain beam elements. The fidelities of these beams are shown in Fig. 5-22, As with the plates, there are beam elements with fidelities greater than 20 that are not shown here. All of these beams meet the minimum fidelity requirement of one. The vibration isolation system contains beams with the lowest fidelity; it has 474 beam elements with fidelities between one and two.

Finally the SM and TM models are examined. The SM and TM are modeled using rigid elements and concentrated masses. The concentrated masses also contain inertia terms. Bittner measures the first flexible mode of the secondary mirror at $2 \mathrm{kHz}$, far above the chosen upper disturbance frequency of 100 $\mathrm{Hz}$. Therefore, it is appropriate to model the SM as a concentrated mass, since its first mode is beyond the frequency range of interest. The fundamental mode of the tertiary mirror assembly was measured at $60 \mathrm{~Hz}$. Since this falls within the frequency range of interest, it is suggested that the TM be modeled instead using flexible finite elements with sufficient fidelity to capture all flexible frequencies below $100 \mathrm{~Hz}$.

The fidelity level of the system is the minimum fidelity across all components, and in this case it is 1.38 , dictated by the VIS plates.

\section{Step 4: Estimate modal frequency error}

The generalized beam and plate equations relating fidelity to modal frequency error are Eqn. 3.12 and 3.25 , respectively. They are used to estimate the modal frequency error of these components and to provide a modal frequency error estimate of the entire system. The modal frequency errors are shown in Figs 5-23 and 5-24 for the plates and beams, respectively, and are broken up according to subsystem.

As expected the plates in the vibration isolation assembly (VIS) with the lowest fidelity levels incur the highest modal frequency errors. It is clear that further mesh refinement is needed in the plates in the VIS and the drive system (DS), since the modal frequency errors are large, over $10 \%$ for these subsystems. A few plates in the scientific instrument flange also do not meet the minimum fidelity requirement. These plates have modal frequency errors of $8.5 \%$.

The beams present in these systems meet the minimum beam fidelity requirement of one. The VIS contains the beams with the lowest fidelity, and their corresponding modal frequency error is $2.42 \%$.

The estimated modal frequency error of the system is estimated as the maximum across components. In this case, since minimum fidelity requirements are not yet met, the modal frequency error cannot yet be 


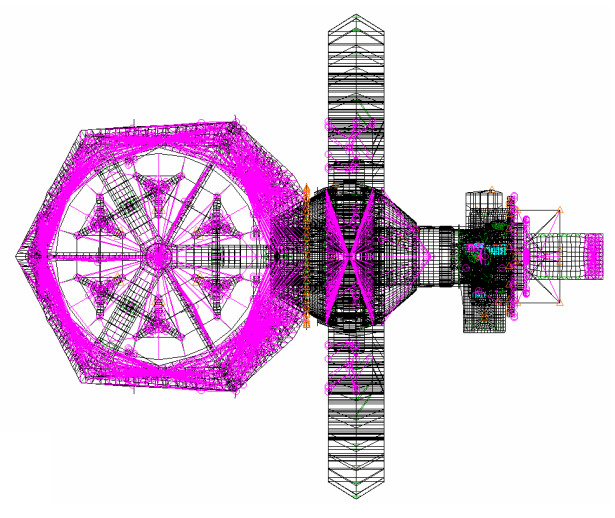

(a) Top view

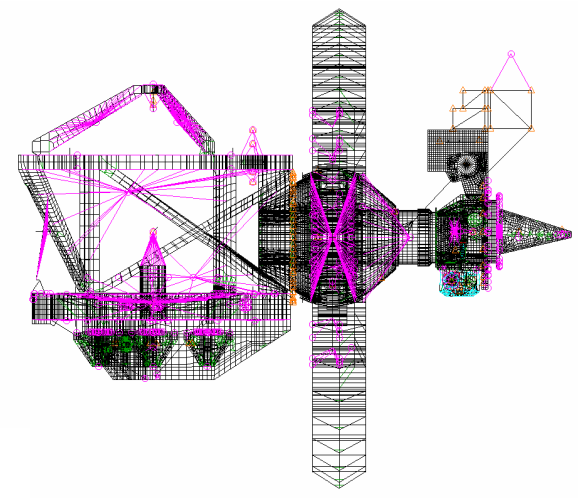

(b) Side view

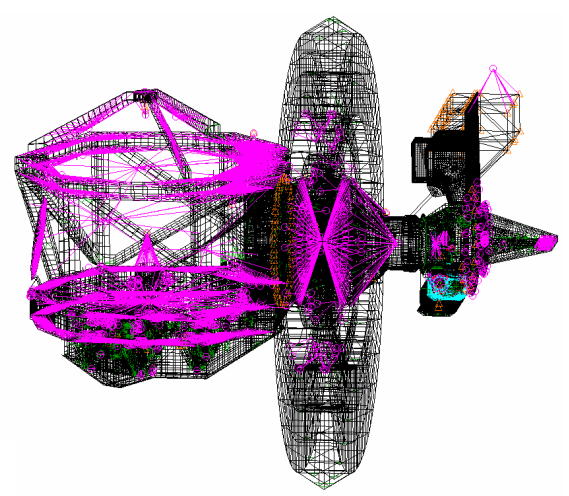

(c) Isometric view

Figure 5-17. SOFIA finite element model 


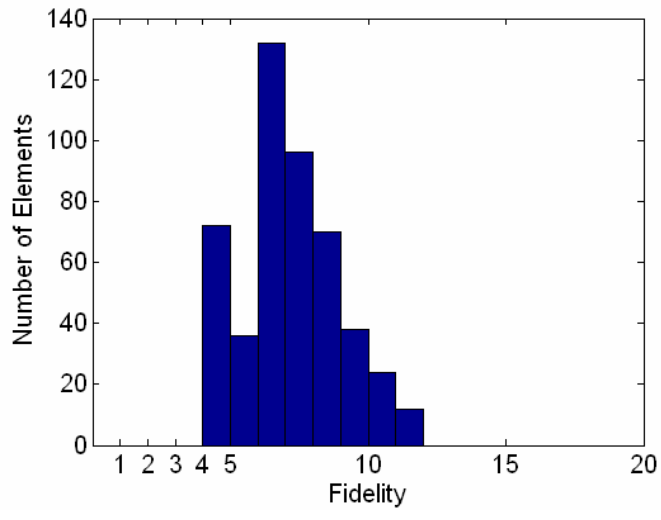

(a) Primary Mirror

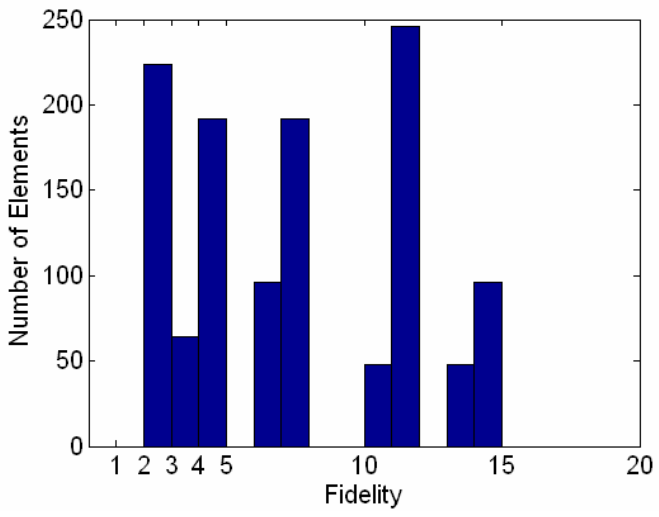

(c) Drive System

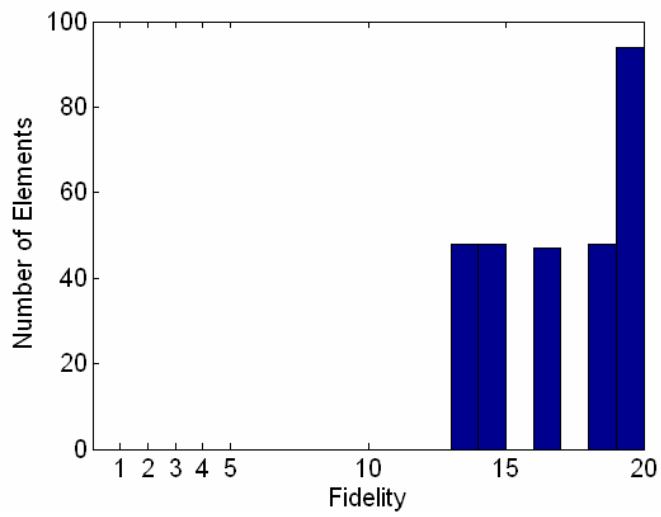

(e) Nasmyth Tube

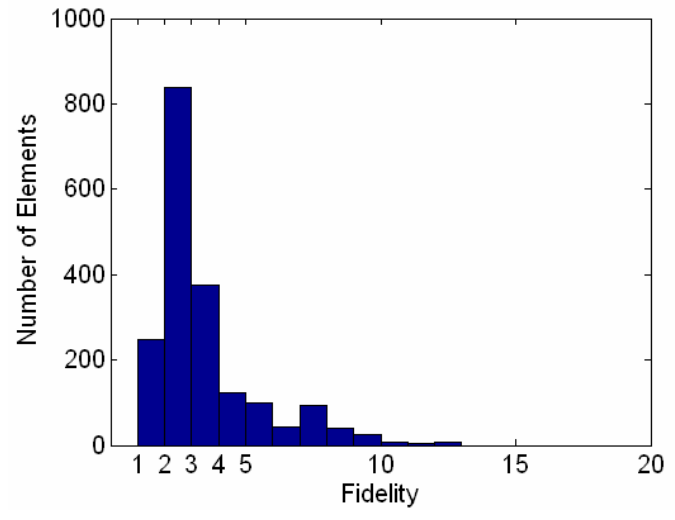

(b) Vibration Isolation System

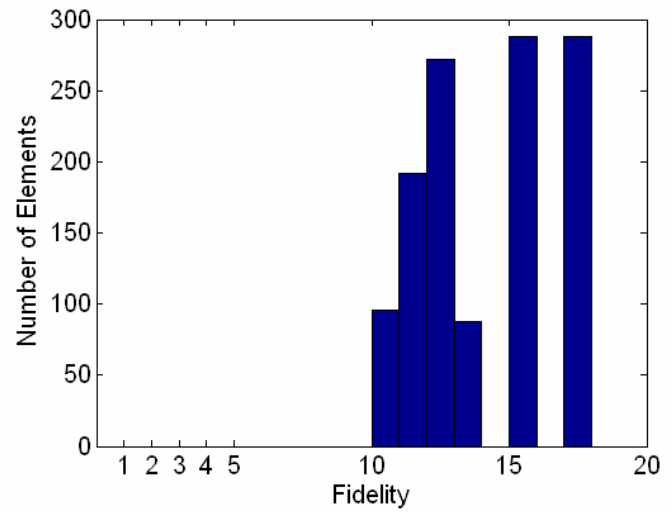

(d) Spherical Bearing

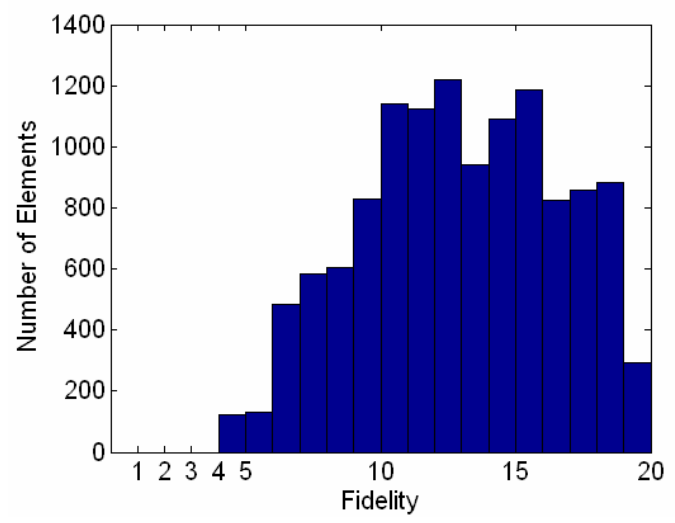

(f) Metering Structure

Figure 5-18. Quantity of plates at each fidelity level for SOFIA subsystems 


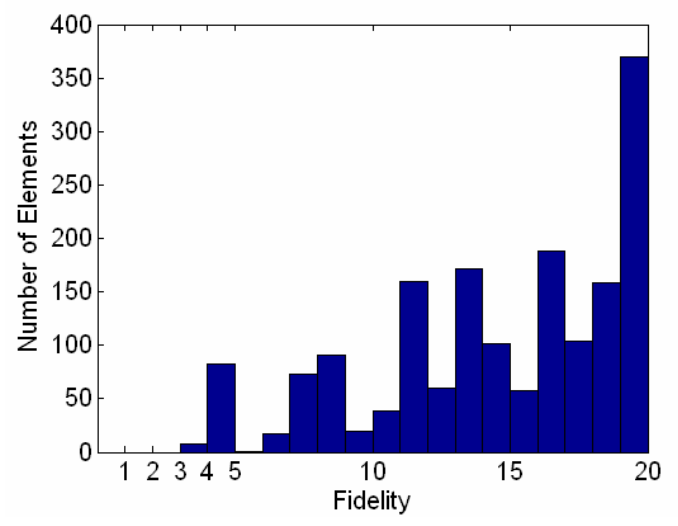

Scientific Instrument Flange

Figure 5-19. Quantity of plates at each fidelity level

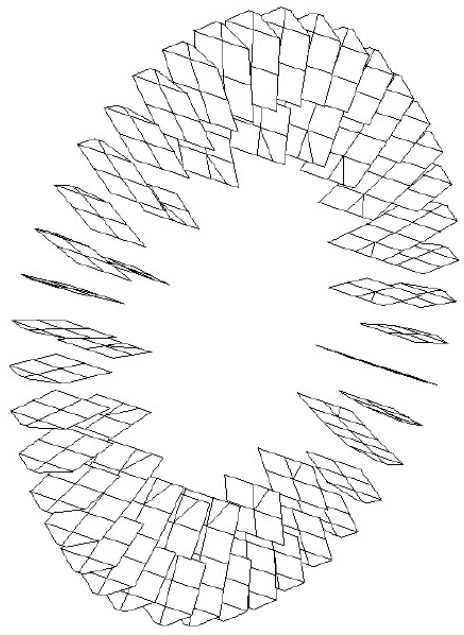

(a) VIS spoke plates

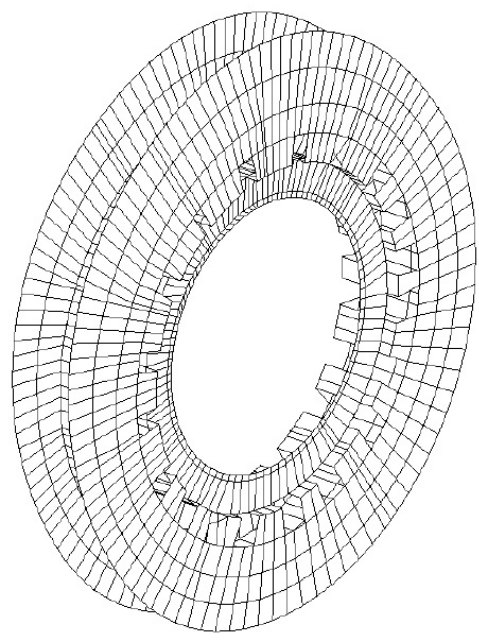

(b) VIS side plates

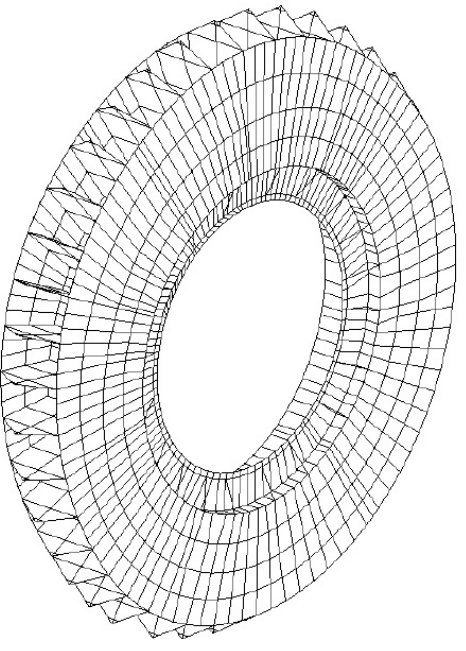

(c) Entire VIS system

Figure 5-20. Vibration Isolation System 


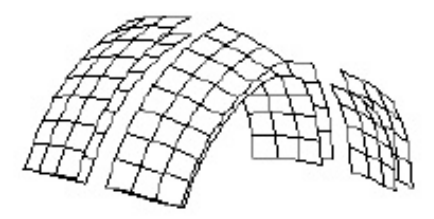

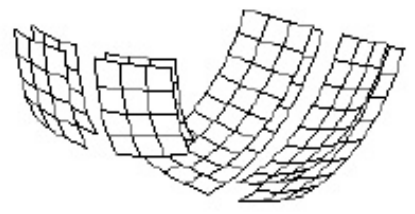

(a) Magnetic Torquer Plates

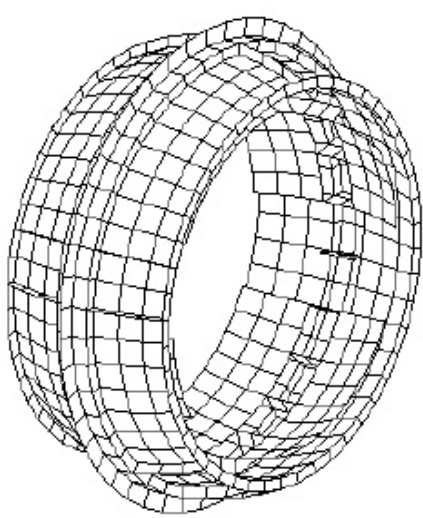

(b) Entire Drive System

Figure 5-21. Drive System

estimated. Further model refinement is required for this estimate.

\subsubsection{SOFIA summary}

The fidelity assessment of the models of the major SOFIA subsystems shown above highlight areas such as the plates in the vibration isolation and drive systems that require further mesh refinement to meet Nyquist requirements and to provide an estimate for the system's modal frequency error. Furthermore, the tertiary mirror assembly is identified as a structure that requires a new model. Its current model consists of a concentrated mass mounted on a rigid element which does not represent the flexible behavior. It was determined that a model with flexible behavior is required.

\subsection{Summary}

The two case studies shown in this chapter, TMT and SOFIA, demonstrate the Nyquist fidelity assessment procedure on real systems with complex models. In both studies, areas of low fidelity in the models were identified as well as areas that were over-meshed. In the TMT study, these were two supporting lattice beams underneath the primary mirror. The error bound estimation procedure revealed that the even though the TMT model meets fidelity requirements, with the exception of the two lattice bars, that the estimated error bound on the output figure of merit is large ( $400 \%$ ) and that further refinement is required to reduce 


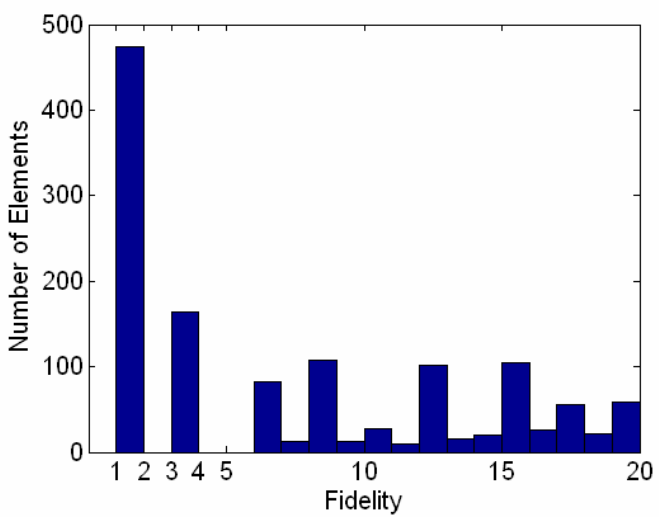

(a) Vibration Isolation System

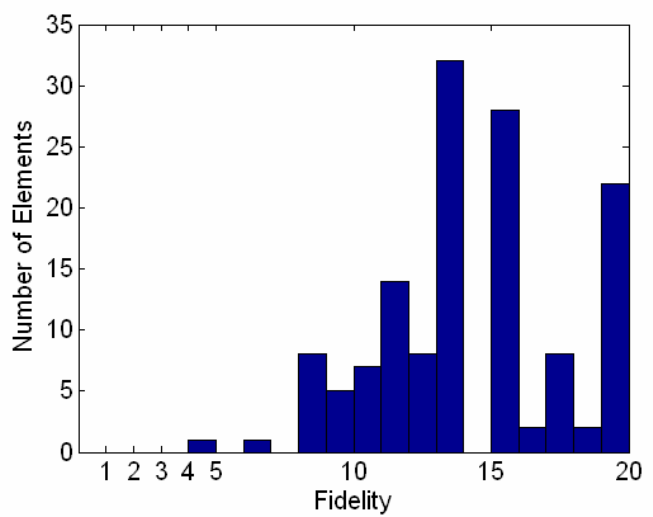

(b) Metering Structure

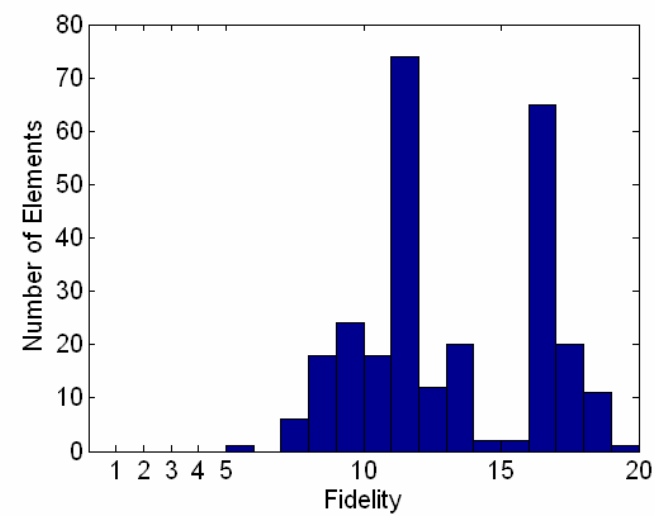

(c) Science Instrument Flange

Figure 5-22. Quantity of beams at each fidelity level 


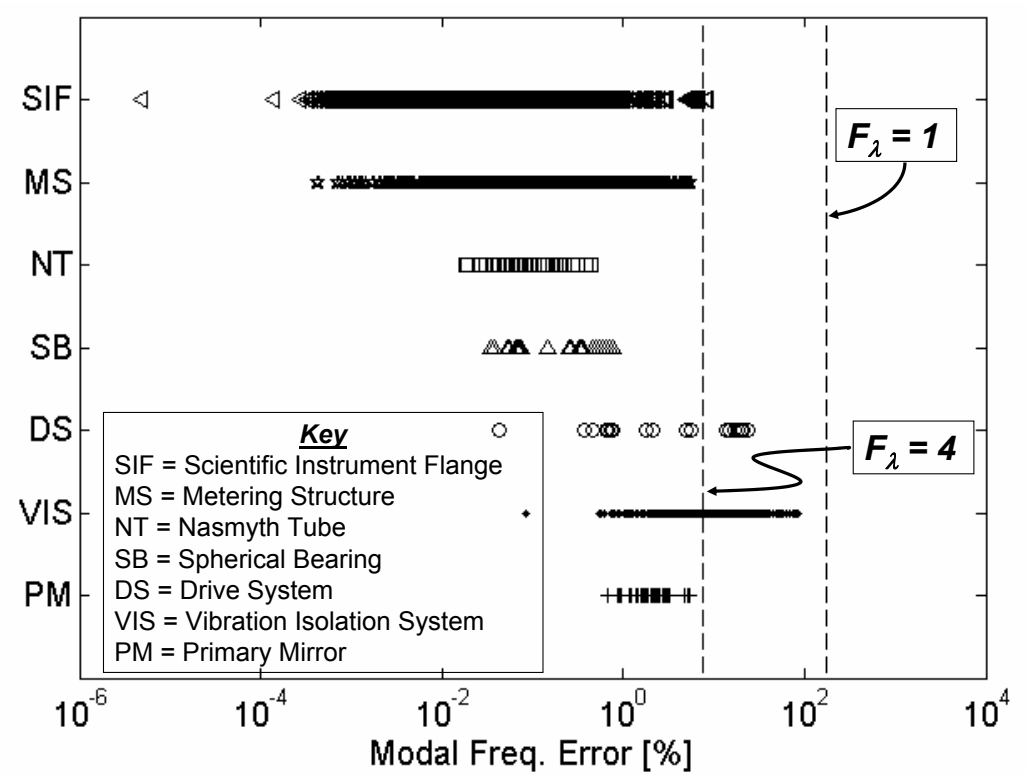

Figure 5-23. Modal frequency error for SOFIA plates

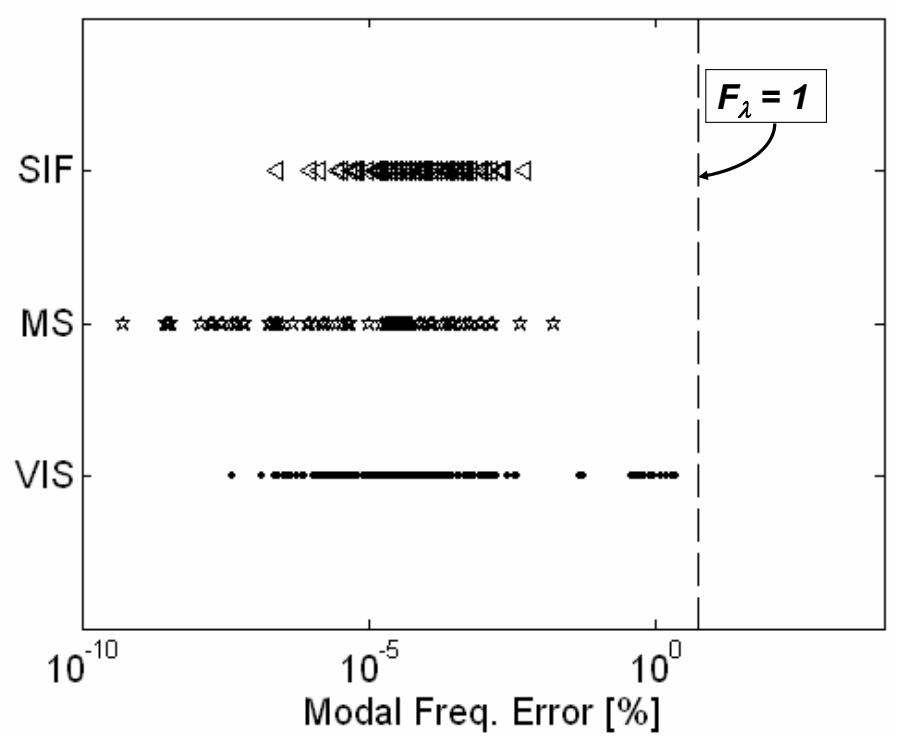

Figure 5-24. Modal frequency error for SOFIA beams 
the error bound to a $2 \%$ level. In the SOFIA study, areas of low fidelity include several plates in the vibration isolation and the drive systems. Additionally, the tertiary mirror assembly is identified as requiring a flexible model representation. 


\section{Chapter 6}

\section{Conclusions and Recommendations}

This chapter contains a thesis summary followed by a listing of the unique contributions of this thesis. A section on model management is presented, which summarizes the best practices for complex modeling based on six years of experience. Finally, a categorized listing of future work recommendations is given.

\subsection{Thesis Summary}

Several precision telescopes in development are due to be completed within the next 10 years. These include the Terrestrial Planet Finder and the Thirty Meter Telescope. These complex opto-mechanical systems are expensive to build and often incorporate new designs and technologies such as lightweight rib-stiffened mirrors and adaptive control of optical surfaces. Since these designs are new, their operating behavior is unknown. Heritage models verified on hardware data cannot be used. Furthermore, the engineering judgement gained from experience with older systems is similarly not directly applicable. In addition, it is expensive to build hardware prototypes of these systems and difficult to simulate operating environments such as micro-gravity and wind gusts.

For these reasons, these systems are ever more reliant on simulation to make design decisions in the conceptual and preliminary design stages. During these stages, there are a large number of design solutions from which to choose and these design decisions greatly impact the cost and performance of the resulting system. These design decisions are made in the absence of hardware data and are based on engineering judgement. 
What is needed is a measure of confidence in the simulation models of new systems that will aid engineers making the conceptual and preliminary design decisions. This thesis provides a quantifiable metric, called the fidelity metric, that measures the fidelity of structural finite element simulation models of telescope systems in the conceptual and preliminary design phases. The fidelity metric is a measure of appropriateness of the finite element model within a larger integrated model. It is not presented as a replacement of the valuable engineering judgement and experience gained on previous systems, but instead is presented as a tool to aid engineers faced with new designs. Also presented in this thesis is a methodology to use this fidelity metric in concert with engineering judgement and experience to build appropriate structural models and to assess the fidelity of existing models. This methodology is called the Nyquist fidelity method.

The simulation environment and modeling methods for integrated models of telescope systems is presented in Chapter 2. This represents the current state-of-the-art in integrated modeling methods. The process for frequency domain analysis, a popular method for model reduction, and the sensitivity analysis procedure are described. These methods offer a brief review of the simulation environment in which the fidelity metric and the Nyquist fidelity method can be applied. It is important to note that linear time invariant systems are considered. An example of a complex integrated model is the Modular Optical Space Telescope (MOST) system, which is composed of structural, optical, controls, and disturbance subsystems. It is a parametric model which automatically generates subsystem models based on architectural design parameters such as focal ratios and control bandwidth. This integrated model is described in detail and the performance analysis procedure is shown. It is important to note that structural models here are constructed using the solution to the homogenous finite element model. The performance analysis gives an estimate of the chosen output figures of merit (OFM). The OFM are generally displacement-based for telescope system models and measure the image degradation due to structural vibrations as a result of disturbance forces.

Next, the fidelity metric is defined and quantified in Section 3.2. The fidelity metric shown in Eqn. 3.2 is a measure of the relative ability of the structural model to represent spatial wavelengths. It is calculated by measuring the finite element mesh size and comparing it to the mesh size required by the spatial Nyquist criterion. It is important that the finite element model has the ability to represent spatial wavelengths since the solution to the homogenous finite element model is used in the integrated model. If the model cannot correctly represent modeshape because the mesh size is too large, the high resonant modeshapes included in 
the homogenous solution would be aliased to lower spatial frequency modes. The resulting displacementbased OFM would be inaccurate since those higher order modeshapes are inaccurate.

The fidelity metric is quantified for beam and plate elements. In the beam case the fidelity metric is given by Eqns. 3.2 and 3.3 A strong relationship between beam fidelity and modal frequency error is observed (Figs. 3-3 and 3-4) and a generalized beam relation is defined (Eqn. 3.12). It is shown that if the Nyquist criterion is met, the beam models can accurately represent spatial modeshapes. If the Nyquist criterion is not met, the modeshapes are aliased as shown in Figs. 3-6 and 3-7. The modal frequency error was calculated using the solution to the analytical model as the "truth" model.

The fidelity of the 2D plate elements is addressed in two ways. First, the Nyquist criterion is used in each dimension to define two companion fidelity metrics in Eqns. 3.18 and 3.19. Since most plates used in telescope system models represent mirrors that are circular or near-circular, the fidelity metrics are defined in the azimuthal and radial directions. A second plate fidelity metric is based on the Nyquist criterion corresponding to a single spatial wavelength number given by wave propagation theory, see Eqn. 3.24 . This is a generalized plate fidelity, applicable to arbitrarily shaped plates. In both plate fidelity metrics, the strong dependence of modal frequency error on fidelity is shown and characterized, see Figs. 3-11, 3-12, 3-19 and Eqn. 3.25 .

An important connection between fidelity and appropriateness of the model is made by estimating error bounds on the chosen output figures of merit (OFM) using the fidelity metric. This is accomplished by using sensitivity analyses to measure the change in the OFM with respect to the change in the structural modal frequencies, introduced in Section 2.2.3. The change in the structural modal frequencies is estimated using the fidelity metric. Combining these methods gives an estimate of the error bounds on the OFM, given in Eqn. 3.5. Once the OFM error bounds are calculated, a decision can be made as to increase or decrease the fidelity level, depending on the desired accuracy of the OFM. Note that this error bound measures the error due to under-meshing of the finite element model. Error in the form of uncertain parameters such as Young's Modulus is not included in this estimate.

Also described in Chapter 3 is the fidelity assessment method. In this method an existing integrated model with known disturbance spectra is assessed. The structural model is represented by a network and the important subsystems are identified. The components in these subsystems are each assigned fidelity 
metric values based on the disturbance spectra. The fidelity metrics translate into modal frequency error measures, which then through using sensitivity analyses can be translated into an error bound estimate on the OFM. The relationship between modal frequency error and the change in OFM is highly dependent on the specific integrated model. An example of the fidelity assessment method is shown by examining the nominal MOST model in Section 3.7. Two structural components in the secondary support tower are identified as having insufficient fidelity to meet minimum Nyquist requirements, see Table 3.7. Ignoring these bars for demonstration purposes, the modal frequency error of the system is estimated at $0.3 \%$. This translates into approximately a $0.31 \%$ error bound in the line-of-sight performance metric.

The procedures developed above are used to construct the Nyquist fidelity method shown in Fig. 4-1. This is an eight step method that begins with an architectural layout of the structural model, a diagram of the integrated model, identifying disturbance sources and determining the OFM. The important subsystems are then identified using the network diagram and the desired modal frequency error is chosen. Then mesh size of the important finite element component models calculated based on the corresponding fidelity metric values. The performance and sensitivity analyses are then run and the OFM error estimate is calculated. This can be an iterative method whereby the modal frequency error is adjusted based on the OFM error bound, the fidelity values are recalculated, the model is re-meshed, and the process repeats.

This method is demonstrated on the MOST model in Section 4.3. A comparison of computational time and OFM accuracy is made between the Nyquist fidelity method and current state-of-the-art method using model reduction. Two levels of modal frequency error are tested, $1 \%$ and 5\%. Significant time savings of $40 \%$ is observed for the Nyquist fidelity method for the 5\% case, see Fig. 4-10, For the $1 \%$ case the Nyquist method actually takes about $80 \%$ longer than the model reduction method, however in both cases, the Nyquist fidelity method is overall more accurate in estimating the OFM, see Fig. 4-11.

Finally, in Chapter 5, two integrated models of real-world telescopes, the Thirty Meter Telescope and the Stratospheric Observatory for Infrared Astronomy are assessed for fidelity in Chapter 5. It is revealed that some structural components do not meet minimum fidelity requirements. These parts are identified and suggestions are made to increase the fidelity of these parts. 


\subsection{Contributions}

The unique and relevant contributions of this thesis advance the field by developing tools to evaluate the appropriateness of integrated models of new and complex opto-mechanical systems. This can be considered the a quantifiable analysis of non-parametric uncertainty of models. The specific contributions are as follows:

- Quantification of fidelity of finite element beam and plate component models based on the satisfaction of the Nyquist criterion for a given finite element mesh and given disturbance spectra.

- Definition of the relationship between fidelity and modal frequency error of structural beam and plate component models. Incorporated sensitivity analysis to relate fidelity to accuracy of displacementbased output figures of merit.

- Development of a method to assess the fidelity of an existing integrated model. Includes identification of important subsystems, definition of system fidelity and estimation of OFM accuracy. Demonstration of fidelity assessment method on three separate telescope models, MOST, TMT and SOFIA.

- Development of a method to create an integrated model with a known level of fidelity that starts geometrical considerations, disturbance spectra, and OFM of interest to generic modeling rules, thereby reducing modeling effort, enabling more models with different configurations to be rapidly developed in conceptual design phase. Demonstration of this method using the MOST model.

- Demonstration of the computational benefits associated with using the Nyquist fidelity method over current methods using the MOST model.

\subsection{Best Practices for Incorporating Fidelity}

The Nyquist fidelity methods presented in thesis are developed as alternative modeling methods for conceptual design structural models of telescopes systems. In the conceptual and preliminary design phases, several configurations of a system are considered. The Nyquist fidelity method generates finite element models with a requested level of fidelity rapidly for each configuration considered. It is designed to be used 
in conjunction with parametric models to evaluate system architectures. The error estimate bound on the OFM due to low fidelity structural models can be added to the uncertainty bounds on the parametric model to make more informed design decisions regarding candidate architectures.

It is important that the amount of fidelity in the system is suitable to the accuracy requirements of the current design phase. Furthermore, for the same model, the model fidelity should increase as the design process progresses. Model tracking, which details the assumptions, configurations, requirements and rationale behind the model's construction, should be employed to track the model fidelity as the design progresses. For example one might have to justify the the mesh size of a component, or change a material property. In this case, the Nyquist fidelity method can be used to justify mesh size.

Once the configurations are narrowed down to a few, more exact methods such as mesh adaptation and balanced reduction can be used in the detailed design phase. In these cases, the Nyquist fidelity method can be used to create a starting mesh for the adaptive mesh method and a high fidelity model for the balanced reduction method.

\subsection{Recommendations for Future Work}

Suggestions for future work to support fidelity methods can be categorized into two main areas: the expansion of the fidelity metric definition and improvements to the Nyquist fidelity method.

\subsubsection{Expanding the fidelity metric definition:}

- In this thesis, the fidelity metric is defined for plate and beam elements. These are common elements in conceptual design models of telescope structural systems, however it would be beneficial to examine other types of commonly used finite elements, such as 3D brick elements.

- Elements containing higher-order interpolation functions should also be examined, since their relationships between fidelity and modal frequency error could be different.

- A few boundary conditions for the beam and plate elements were examined. Other common boundary conditions, such as the bipod mount of a mirror, could be explored. 
- The fidelity metric denominator is based on the maximum disturbance frequency of the system. Future work could include a determination of the appropriate frequency to used here.

- The effects of using a lumped mass matrix over a consistent mass matrix was also not examined here. Since mass lumping reduces computational time, it would be useful to quantify its effect on fidelity.

- In the plate case, an investigation into the violation of the generalized plate fidelity relation by the first mode of the hexagonal plate can be made.

- The two proposed fidelity metrics for the plate, azimuthal/radial and generalized, do not track each other well. This difference could be reconciled

\subsubsection{Improving the Nyquist fidelity method}

- The Nyquist methods presented in this thesis give a step by step roadmap on how to incorporate fidelity quantification into the modeling process. If these methods were automated in a software tool, they would be more accessible to a wider audience. It would also reduce the model set-up time.

- These methods could also be combined with existing parametric uncertainty methods to develop more robust and reliable designs in the conceptual and preliminary design phases.

- In the error estimation process, the sensitivity analysis results are used to estimate error bounds. It would be useful to evaluate the range of validity (linearity and conditioning) of the sensitivity results and incorporate those ranges into the error bound estimation, thereby making the error bounds would be more accurate.

- An assumption made during the Nyquist fidelity method was to model appendages with sufficient fidelity to capture their first fundamental mode. The effects of appendage modes on the coupled system modes could be evaluated, perhaps using component mode synthesis techniques. This could result in a new way of dealing with appendage substructures within the Nyquist fidelity method.

- In this work, it is assumed that the component (beam and plate) modal accuracies translate into coupled system modal accuracies. Further investigation into the effects on modal accuracy of coupling 
subsystem models can be done. This could use the quasi-static response to determine finer meshing at the interfaces. 


\section{Appendix A}

\section{Analytical Beam equations}

\section{A.1 Beam Analytical Solution}

The differential equation for a homogenous isotropic beam is [59]:

$$
\frac{d^{2}}{d x^{2}}\left[E I(x)\left(\frac{d^{2} W(x)}{d x^{2}}\right)\right]-\frac{d}{d x}\left[P(x) \frac{d W(x)}{d x}\right]=\lambda m(x) W(x) \lambda=\omega^{2}
$$

where $\mathrm{W}(\mathrm{x})$ is the transverse displacement, $\mathrm{P}(\mathrm{x})$ is the axial force and $\mathrm{m}(\mathrm{x})$ is the mass density. And $\mathrm{W}(\mathrm{x})$ must satisfy either

$$
-\frac{d}{d x}\left[E I(x)\left(\frac{d^{2} W(x)}{d x^{2}}\right)\right]+P(x) \frac{d W(x)}{d x}=0 \text { at } \mathrm{x}=0, \mathrm{~L}
$$

or

$$
W(x)=0 \text { at } \mathrm{x}=0, \mathrm{~L}
$$

and either

$$
E I(x) \frac{d^{2} W(x)}{d x^{2}}=0 \text { at } \mathrm{x}=0, \mathrm{~L}
$$

or

$$
\frac{d W(x)}{d x}=0 \text { at } \mathrm{x}=0, \mathrm{~L}
$$




\section{A.2 Pinned-pinned Uniform Beam, no axial force}

For a pinned-pinned uniform beam with no axial force, the resonant (modal frequencies) are [59]:

$$
\omega_{r}=(\mathrm{r} \pi)^{2} \sqrt{\frac{E I}{m L^{4}}}, r=1,2, \ldots
$$

The modeshapes are given as:

$$
W_{r}(x)=\sqrt{\frac{2}{m L}} \sin \frac{r \pi x}{L}, r=1,2, \ldots
$$

and the eigenvalues are normalized such that:

$$
\int_{0}^{L} m W_{r}^{2} d x=1
$$

where $r$ is the mode number, $\mathrm{E}$ is Young's modulus $[\mathrm{Pa}], \mathrm{I}$ is moment of inertia $\left[\mathrm{m}^{4}\right], m$ is mass per unit length $[\mathrm{kg} / \mathrm{m}]$, and $L$ is beam length $[\mathrm{m}]$.

\section{A.3 Cantilever Uniform Beam}

For a cantilever uniform beam, the modal frequencies are [59]:

$$
\cos \beta L \cosh \beta L=-1
$$

where $\beta^{4}=\frac{\omega^{2} m}{E I}$.

The modeshapes are

$$
\begin{aligned}
W_{r}(x)= & A_{r}\left[\left(\sin \beta_{r} L-\sinh \beta_{r} L\right)\left(\sin \beta_{r} x-\sinh \beta_{r} x\right)+\right. \\
& \left.\left(\cos \beta_{r} L-\cosh \beta_{r} L\right)\left(\cos \beta_{r} x-\cosh \beta_{r} x\right)\right], \mathrm{r}=1,2, \ldots
\end{aligned}
$$


where $A_{r}=C_{1} /\left(\sin \beta_{r} L-\sinh \beta_{r} L\right)$ and $C_{1}$ is a constant of integration. Here, the eigenvalues are not normalized.

\section{A.4 Free-free Unform Beam}

For the free-free uniform beam, there are two rigid body modes, one translation and one rotation about the center of the beam. The characteristic equation gives the modal frequencies and is [59]:

$$
\cos \beta L \cosh \beta L=1
$$

The modeshapes are given as:

$$
\begin{aligned}
W_{r}(x)= & A_{r}\left[\left(\cos \beta_{r} L-\cosh \beta_{r} L\right)\left(\sin \beta_{r} x-\sinh \beta_{r} x\right)-\right. \\
& \left(\sin \beta_{r} L-\sinh \beta_{r} L\right)\left(\cos \beta_{r} x-\cosh \beta_{r} x\right), \mathrm{r}=2,3, \ldots
\end{aligned}
$$

\section{A.5 Beam Wave Number}

For a Bernouli-Euler beam, the wave number based on frequency is [28]:

$$
k= \pm\left[\frac{\omega^{2} \rho A-i \omega \eta A}{E I}\right]^{1 / 4}
$$

The corresponding spatial wavelength is

$$
\lambda=\frac{2 \pi}{k}
$$




\section{Appendix B}

\section{Analytical Plate Equations}

\section{B.1 Analytical Plate Equations}

The governing differential equation of a thin plate in rectangular coordinates $(x, y)$ using classical smalldeflection theory is [76],

$$
\frac{\partial^{4} w}{\partial x^{4}}+2 \frac{\partial^{4} w}{\partial x^{2} \partial y^{2}}+\frac{\partial^{4} w}{\partial y^{4}}=\frac{p_{z}(x, y)}{D}
$$

where $w$ is the transverse displacement and $D$ is the flexural rigidity.

$$
D=\frac{E h^{3}}{12\left(1-v^{2}\right)}
$$

Classical small-deflection plate theory assumes an originally flat, constant thickness plate made of material that follows Hooke's law (is homogenous, isotropic and linear), and whose middle lateral surface does not undergo strain during bending. Equation B.1 is also called the nonhomogenous biharmonic equation. In polar coordinates, the biharmonic equation becomes [76],

$$
\nabla_{r}^{2} \nabla_{r}^{2} w=\frac{p_{s}(r, \varphi)}{D}
$$

where $\nabla_{r}^{2}$ is the Laplacian operator,

$$
\nabla_{r}^{2}=\frac{\partial^{2}}{\partial r^{2}}+\frac{1}{r^{2}} \frac{\partial^{2}}{\partial \varphi^{2}}+\frac{1}{r} \frac{\partial}{\partial r}
$$




\section{B.2 Circular Plate}

For a circular plate, the transverse displacement of resonant modes in polar coordinates is given as

$$
\mathrm{Z}(\mathrm{r}, \theta, \mathrm{t})=\left[\mathrm{a}_{\mathrm{i}} \mathrm{J}_{\mathrm{i}}\left(\frac{\lambda \mathrm{r}}{\mathrm{a}}\right)+\mathrm{b}_{\mathrm{i}} \mathrm{Y}_{\mathrm{i}}\left(\frac{\lambda \mathrm{r}}{\mathrm{a}}\right)+\mathrm{c}_{\mathrm{i}} J_{\mathrm{i}}\left(\frac{\lambda \mathrm{r}}{\mathrm{a}}\right)+\mathrm{d}_{\mathrm{i}} \mathrm{K}_{\mathrm{i}}\left(\frac{\lambda \mathrm{r}}{\mathrm{a}}\right)\right] \cos \mathrm{i} \theta \sin 2 \pi \mathrm{ft}
$$

where $\mathrm{f}$ is the natural frequency given by

$$
\mathrm{f}=\frac{\lambda^{2}}{2 \pi \mathrm{a}^{2}}\left[\frac{\mathrm{Eh}^{3}}{12 \gamma\left(1-\nu^{2}\right)}\right]^{1 / 2}
$$

and

- $\mathrm{J}_{\mathrm{i}}$ - Bessel function of the first kind

- $J_{\mathrm{i}}$ - modified Bessel of the first kind

- $\mathrm{Y}_{\mathrm{i}}-$ Bessel function of the second kind

- $\mathrm{K}_{\mathrm{i}}$ - modified Bessel of the second kind

The terms $\mathrm{Y}$ and $\mathrm{K}$ are only present in elliptical or annular plates. Therefore the modeshapes for a circular plate are [14]:

$$
\tilde{z}_{i j}(r, \theta)=\left[\mathrm{a}_{\mathrm{ij}} \mathrm{J}_{\mathrm{i}}\left(\frac{\lambda_{\mathrm{ij}} \mathrm{r}}{\mathrm{a}}\right)+\mathrm{b}_{\mathrm{ij}} \mathrm{J}_{\mathrm{i}}\left(\frac{\lambda_{\mathrm{ij}} \mathrm{r}}{\mathrm{a}}\right)\right] \operatorname{cosi} \theta
$$

For a simply supported (along the edge) circular plate, $\lambda$ is

$$
\frac{\mathrm{J}_{\mathrm{i}+1}(\lambda)}{\mathrm{J}_{\mathrm{i}}(\lambda)}+\frac{J_{\mathrm{i}+1}(\lambda)}{J_{\mathrm{i}}(\lambda)}=\frac{2 \lambda}{1-\nu}
$$

and the modeshape is:

$$
\left[\mathrm{J}_{\mathrm{i}}\left(\frac{\lambda \mathrm{r}}{\mathrm{a}}\right)-\frac{\mathrm{J}_{\mathrm{i}}(\lambda)}{J_{\mathrm{i}}(\lambda)} J_{\mathrm{i}}\left(\frac{\lambda \mathrm{r}}{\mathrm{a}}\right)\right] \cos \mathrm{i} \theta, \mathrm{i}=0,1,2, \ldots
$$




\section{B.3 MAC Plots for Circular Plate}

The plots shown below in Figs. B-1 and B-2 are the modal assurance criterion (MAC) plots of the first ten modes of the circular plate described in Section 3.4.1.

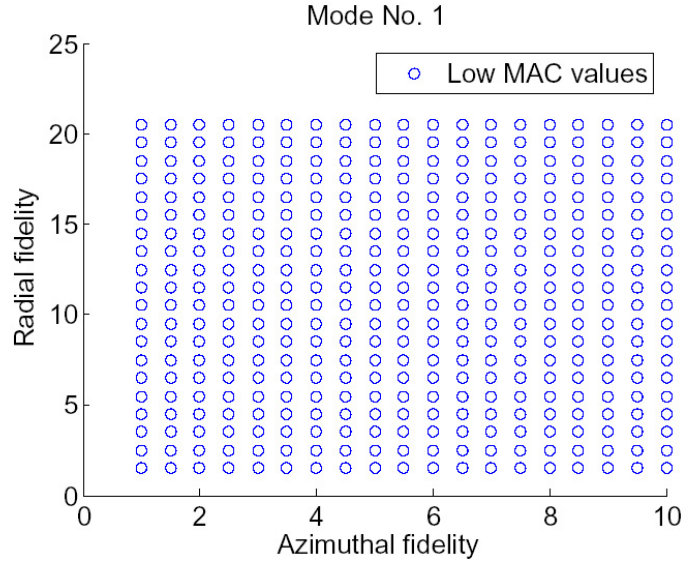

(a) Mode 1

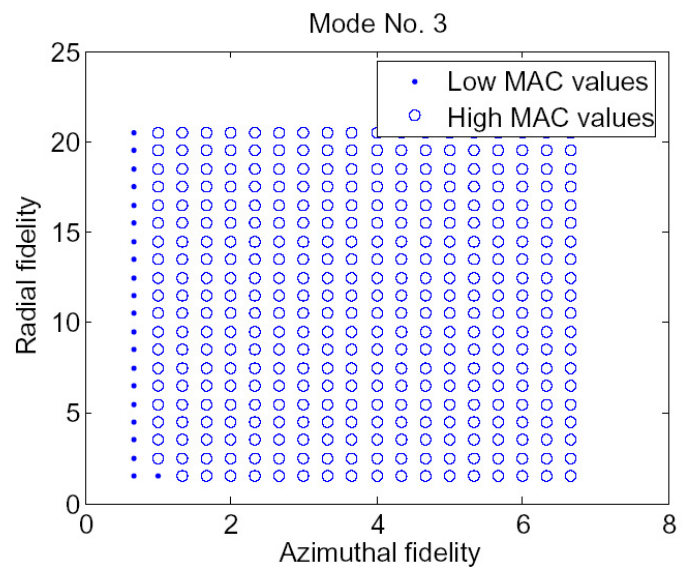

(c) Mode 3

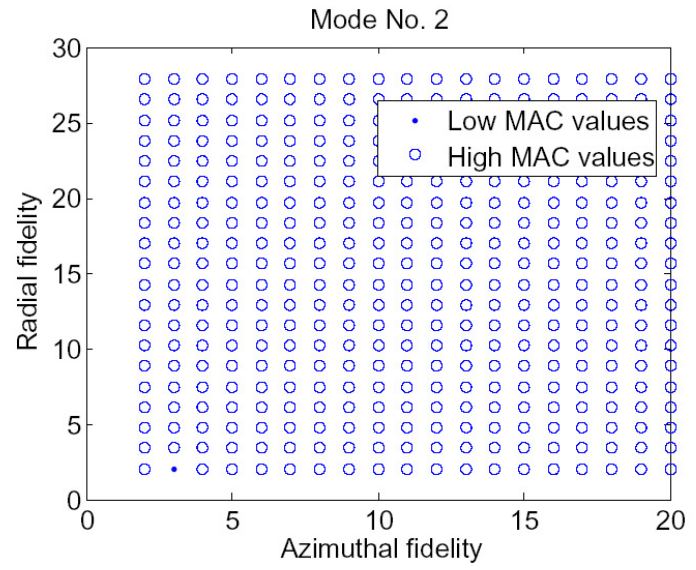

(b) Mode 2

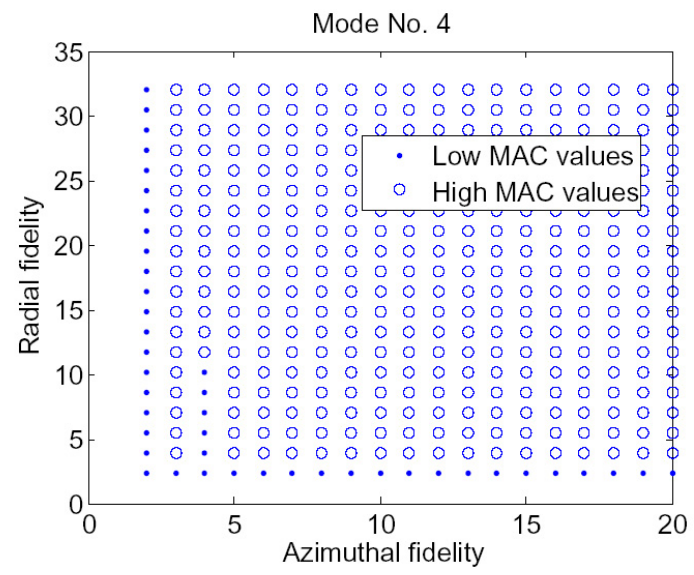

(d) Mode 4

Figure B-1. MAC values for the first 10 modes of a circular monolithic free free plate 


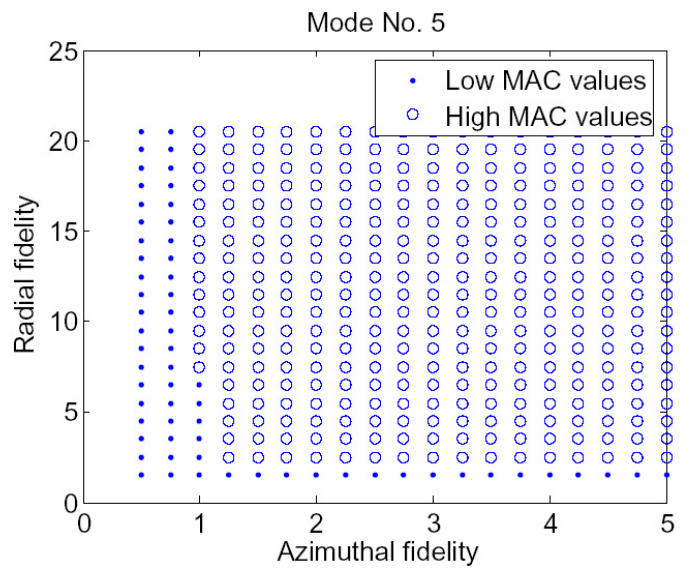

(e) Mode 5

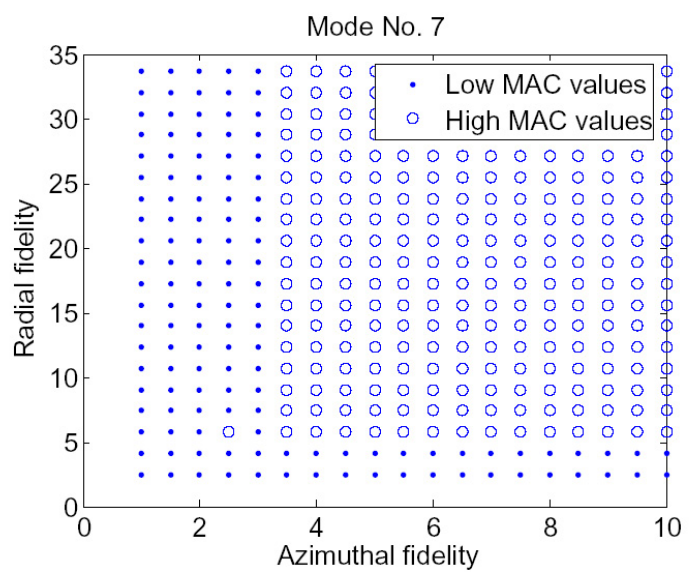

(g) Mode 7

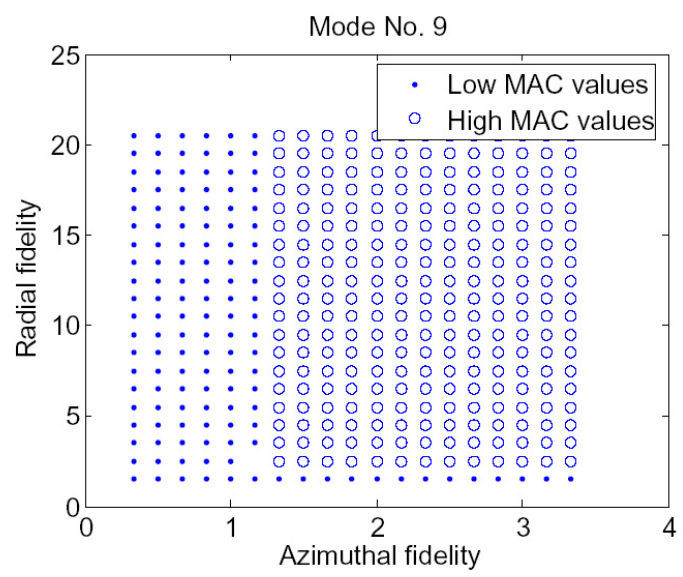

(i) Mode 9

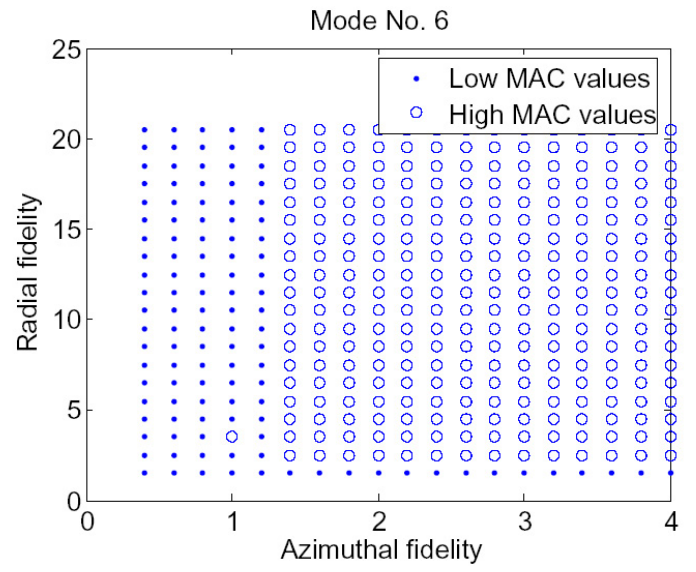

(f) Mode 6

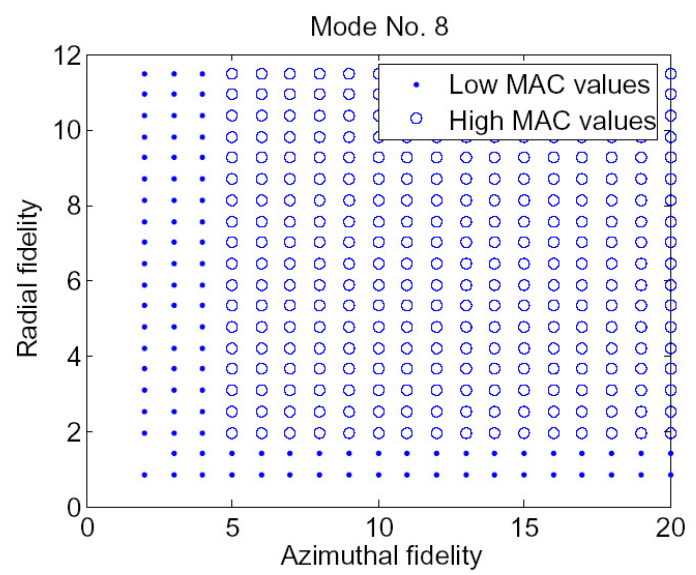

(h) Mode 8

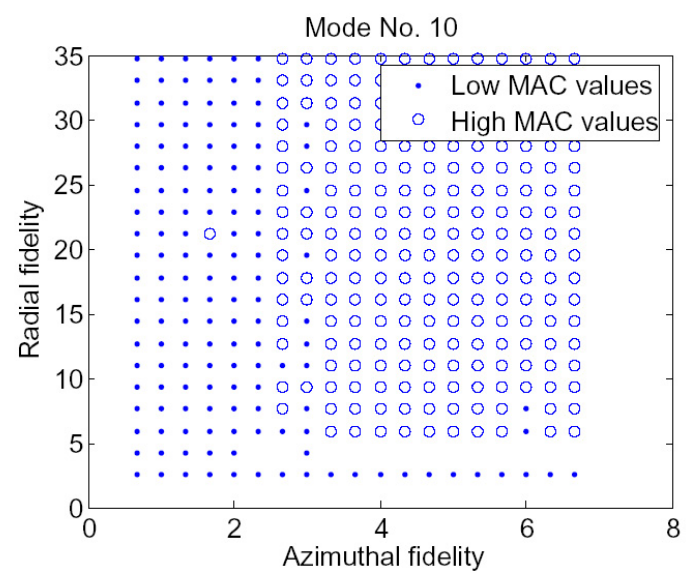

(j) Mode 10

Figure B-2. MAC values for the first 10 modes of a circular monolithic free free plate 


\section{Bibliography}

[1] Astronomy and Astrophysics in the New Millenium. National Academy Press, 2001.

[2] Structural vibration modeling and verification technology exchange forum. Technical report, Aerospace Corp., El Segundo, CA, April 2005.

[3] J.D. Achenbach. Wave propagation in Elastic Solids. North Holland Publishing Company, 1973.

[4] N.M. Alexandrov, R.M. Lewis, C.R. Gumbert, L.L. Green, and P.A. Newman. Approximation and model management in aerodynamic optimization with variable-fidelity models. Journal of Aircraft, 38(6):1093-1101, 2001.

[5] R.J. Allemang. Investigation of some multiple input/output frequency response function experimental modal analysis techniques. $\mathrm{PhD}$ thesis, Cincinnati University.

[6] George Angeli and Brooke Gregory. Linear optical model for a large ground based telescope. In Proceedings of SPIE Volume: 5178 Optical Modeling and Performance Predictions, January 2004.

[7] George Z. Angeli, Robert S. Upton, Anna Segurson, and Brent L. Ellerbroek. Active optics challenges of a thirty-meter segmented mirror telescope. In Proceedings of SPIE Volume: 5382 Second Backaskog Workshop on Extremely Large Telescopes, July 2004.

[8] A. Antoulas and D. Sorensen. Approximation of large-scale dynamical systems: An overview. International Journal of Applied Mathematics and Computational Science, 11:1093-1121, 2001.

[9] Association of Universities for Reserach in Astronomy New Initiaitves Office. TMT project. http://www.aura-nio.noao.edu/. 
[10] Ivo Babuska. The finite element method and its reliability. Clarendon Press, 2001.

[11] Klaus-Jürgen Bathe. Finite Element Procedures. Prentice Hall, 1996.

[12] Klaus-Jürgen Bathe, Nam-Sua Lee, and Miguel Luiz Bucalem. On the use of hierarchical models in engineering analysis. Computer Methods in Applied Mechanics and Engineering, 82, 1990.

[13] Hermann Bittner, Matthias Erdmann, Markus Erhard, and Peter Haberler. The Optical System of the SOFIA Telescope. Proc.SPIE Ground based Telescopes, 5489:805-816, Sept. 2004.

[14] Robert D. Blevins. Formulas for Natural Frequency and Mode Shape. Robert E. Krieger Publishing Company, 1984.

[15] A.J. Booth et al. Overview of the control system for the Keck interferometer. Proceedings of the SPIE, SPIE-4848:107-115, 2002.

[16] Max Born and Emil Wolf. Principles of Optics. Cambridge University Press, 1999.

[17] Robert Brown and Patrick Hwang. Introduction to Random Signals and Applied Kalman Filtering. John Wilely \& Sons, 3rd edition, 1997.

[18] Kwan J. Chang, Raphael T. Haftka, Gary L. Giles, and Pi-Jen Kao. Sensitivity-based scaling for approximating structural response. Journal of Aircraft, 30(2):283-288, March-April 1993.

[19] Lucy Cohan. Integrated modeling to facilitate control architecture design for lightweight space telescopes. Master's thesis, MIT, 2007.

[20] David A. Content and Virginia Ford. Engineering Studies of the TPF-Coronagraph Telescope. AIAA Space Conference, 2005. AIAA Paper 2005-6673.

[21] R.D. Cook and J. Avrashi. Error estimation and adaptive meshing for vibration problems. Computers \& Structures, 44(3):619-626, 1992.

[22] Roy R. Craig. Coupling of substructures for dynamic analysis: An overview. In 41st AIAA/ASME/ASCE/AHS/ASC Structures, Structural Dynamics, and Materials Conference and Exhibit, 2000. AIAA Paper 2000-1573. 
[23] L.P. Davis, J.F. Wilson, R.E. Jewell, and J.J. Roden. Hubble space telescope reaction wheel assembly vibration isolation system. Vibration Damping Workshop, March 1986. NASA Marshall Space Flight Center.

[24] Olivier de Weck. Integrated modeling and dynamics simulation for the next generation space telescope. Master's thesis, MIT, 1999.

[25] Olivier de Weck. Multivariable Isoperformance Methodology for Precision Opto-Mechanical Systems. PhD thesis, MIT, 2001.

[26] Olivier L. de Weck, David Miller, and Gary E. Mosier. Multivariable isoperformance methodology for precision opto-mechanical systems. In 43rd AIAA/ASME/ASCE/AHS/ASC Structures, Structural Dynamics, and Materials Conference, 2002. AIAA Paper 2002-1420.

[27] Olivier L de Weck, David W. Miller, Gregory Mallory, and Gary E. Mosier. Integrated modeling and dynamics simulation for the Next Generation Space Telescope (NGST). In UV, optical, and IR space telescopes and instruments Conference, 2000. SPIE Proceedings. Vol. 4013.

[28] James F. Doyle. Wave Propagation in Structures. Springer, 1997.

[29] A. Dutta, C.V. Ramakrishnan, and P. Mahajan. Adaptive finite element analysis of structures under transient dynamic loading using modal superposition. Finite Elements in Analysis and Design, 31:255$272,1999$.

[30] M.S. Eldred, A.A. Giunta, and S.S. Collis. Second-Order Corrections for Surrogate-Based Optimization with Model Heirarchies. In Proceedings of the 10th AIAA/ISSMO Multidisciplinary Analysis and Optimization Conference, 2004. AIAA 2004-4457.

[31] K. Gallivan, E. Grimme, and P. Van Dooren. Pade approximation of large-scale dynamic systems with Lanczos methods. In Proceedings of the 33rd IEEE Conference on Decision and Control, pages 443-448, 1994.

[32] Wodek Gawronski. Dynamics and Control of Structures. Springer, 1998. 
[33] Wodek Gawronski. Advanced structural dynamics and active control of structures. New York Springer, 2004.

[34] Goddard Space Flight Center, Systems Engineering Division. ”The NASA Mission Design Process", 1992.

[35] Joseph W. Goodman. Introduction to Fourier Optics. McGraw-Hill, 1996.

[36] Thomas Grätsch and Klaus-Jürgen Bathe. A posteriori error estimation techniques in practial finite element analysis. Computers \& Structures, 83(2):235-265, 2004.

[37] Robert L. Grogan and Robert A. Laskin. On multidisciplinary modeling of the space interferometry mission. American Control Conference,, 1998.

[38] G.P. Guruswamy. Use of high fidelity methods in multidisciplinary optimization - a preliminary study. In 9th AIAA/ISSMO Symposium on Multidisciplinary Analysis and Optimization, 2002.

[39] Homero L. Gutierrez and David W. Miller. Performance Assessment and Enhancement of Precision Controlled Structures During Conceptual Design. PhD thesis, Massachusetts Institute of Technology, February 1999.

[40] Franziska Harms, Patrick Waddell, Martin Süss, and Hans-Peter Röser. On sky testing and preliminary sensor alignment for the SOFIA telescope. In SPIE Astronomical Telescopes and Instrumentation, 2006.

[41] Thomas Hughes. The finite element method : linear static and dynamic finite element analysis. Prentice-Hall, 1987.

[42] W.C. Hurty. Dynamic analysis of structural systems using component modes. AIAA Journal, 3(4):678$685,1965$.

[43] S. Joshi, J. Melody, and G. Neat. A case study of the role of structural/optical model fidelity in performance prediction of complex opto-mechanical instruments. In Proceedings of the 36th Conference on Decision Control, 1997. 
[44] Hans J. Kärcher. Airborne environment - a challenge for telescope design. In SPIE Astronomical Telescopes and Instrumentation, 2000.

[45] A. Krabbe and H. Kaercher. Preparing for first light: The SOFIA telescope. In SPIE Conference on Astronomical Telescopes and Instrumentation, volume 5495, June 2004.

[46] A. Laub, M. Heath, C. Paige, and R. Ward. Computation of system balancing transformations and other applications of simultaneous diagonalization algorithms. IEEE Transactions on Automatic Control, AC-32(2):115-122, 1987.

[47] Oliver P. Lay et al. Architecture trade study for the terrestrial planet finder interferometer. Proceedings of the SPIE, SPIE-5905:8-20, 2005.

[48] Arthur W. Leissa. Vibration of Plates. Office of Technology Utilization, NASA, 1969. NASA Report: SP-160.

[49] T. Lieu, C. Farhat, and M. Lesoinne. Reduced-order fluid/structure modeling of a complete aircraft configuration. Computational Methods in Applied Mechanical Engineering, 195(41-43):5730-5742, 2006.

[50] David LoBosco, Carl Blaurock, Soon-Jo Chung, and David W. Miller. Integrated modeling of optical performance for the terrestrial planet finder structurally connected interferometer. In Modeling and Systems Engineering for Astronomy, 2004.

[51] J. L. Lumely. The structures of inhomogeneousturbulent flow. In A. M. Yaglom and V. I. Tatarski, editors, Atmospheric Turbulence and Radio Wave Propagation, pages 166-178, Nauka, Moscow, 1967.

[52] Doug MacMartin and Gary Chanan. Control of the California extremely large telescope primary mirror. SPIE Future Giant Telescopes Conference, 2002.

[53] Douglas G. MacMynowski, George Z Angeli, Konstantinos Vogiatzis, Joeleff Fitzsimmons, and Stephen Padin. Parametric modeling and control of telescope wind-induced vibration. SPIE Astronomical Telescopes Conference, pages 266-277, 2004. 
[54] Douglas G. MacMynowski, Carl Blaurock, and George Z. Angeli. Initial control results for the thirty meter telescope. AIAA Guidance, Navigation, and Control Conference and Exhibit, 2005. AIAA Paper 2005-6075.

[55] J.R. Maly, P.J. Keas, and R.M. Glaese. Damping SOFIA:passive and active damping for the stratospheric observatory for infrared astronomy. In SPIE Smart Structures and Materials Conference, volume 4331, 2001.

[56] Rebecca Masterson. Dyanmic Tailoring and Tuning for Space-Based Precision Optical Structures. PhD thesis, MIT, 2005.

[57] Rebecca A. Masterson and David W. Miller. Dynamic tailoring and tuning for precision optical space structures. In 45th AIAA/ASME/ASCE/AHS/ASC Structures, Structural Dynamics and Materials Conference, 2004.

[58] Mathworks Inc. Matlab Reference Manual.

[59] Leonard Meirovitch. Principles and Techniques of Vibration. Prentice Hall, 1996.

[60] D. W. Miller, O. L. de Weck, and G. E. Mosier. Framework for multidisciplinary integrated modeling and analysis of space telescopes. In Integrated Modeling of Space Telescopes, number SPIE-4757, 2002.

[61] D W Miller, O.L de Weck, S.A. Uebelhart, R. Grogan, and Basdogan I. Integrated dynamics and controls modeling for the Space Interferometry Mission (SIM). In 2001 IEEE Aerospace Conference, Big Sky, MT, 2001.

[62] D.W. Miller and Simon Grocott. Robust control of the multiple mirror telescope adaptive secondary mirror. Optical Engineering, 38(8):1276-1287, 1999.

[63] Douglas C. Montgomery. Design and Analysis of Experiments. John Wiley and Sons, 3rd edition.

[64] B.C. Moore. Principal component analysis of linear systems: Controllability, observability, and model reduction. IEEE Transactions on Autmatic Control, AC-26(1):17-32, 1981. 
[65] MSC.Software Corporation. MSC.Nastran Reference Manual,, 2004.

[66] C.T. Mullis and R.A. Roberts. Synthesis of minimum roundoff noise fixed point digital filters. IEEE Transactions on Circuits and Systems, CAS-23:551-562, 1976.

[67] National Aeronautics and Space Administration, Washington, DC. NASA Standard i7009: Standards for Models and Simulations, December 2006.

[68] Alan V. Oppenheim, Ronald Schafer, and John Buck. Discrete-time signal processing. Prentice Hall, 2nd edition, 1999.

[69] Sybil P. Parker, editor. McGraw-Hill Dictionary of Scientific and Technical Terms. McGraw-Hill, fifth ed. edition, 1994.

[70] C. Perrygo and R. Burg. Hand calculation of line-of-sight senstivity to optics displacements. Technical report, NGST Systems Engineering memorandum, January 1999.

[71] N.V. Queipo, R.T. Haftkaa, W. Shyya, T. Goela, R. Vaidyanathan, and P. K. Tucker. Surrogate-based analysis and optimization. Progress in Aerospace Sciences, 41(1):1-28, Jan. 2005.

[72] T.D. Robinson, K.E. Willcox, M.S. Eldred, and R. Haimes. Multifidelity optimization for variablecomplexity design. 11th AIAA/ISSMO Multidisciplinary Analysis and Optimization Conference, (AIAA 2006-7114), 2006.

[73] Phillip A. Sabelhaus et al. An overview of the James Webb Space Telescope (JWST) project. Proceedings of the SPIE, SPIE-5899:241-254, 2005.

[74] Ulrich Schönhoff, Peter Eisenträger, Karl Wandner, Hans Kärcher, Ranier Nordmann, and Martin Süss. End-to-end simulation of the image stability for the airborne telescope SOFIA. In SPIE Astronomical Telescopes and Instrumentation, 2000.

[75] Martin Süess, Karl Wandner, Hans Kärcher, Peter Eisenträger, and Ulrich Schönhoff. Airborne pointing and pointing improvement strategy for SOFIA. In SPIE Astronomical Telescopes and Instrumentation, volume 4857, 2002. 
[76] Rudolph Szilard. Theories and Applications of Plate Analysis. John Wilely \& Sons, 2004.

[77] Scott Uebelhart, D.J. Howell, and D.W. Miller. Evaluating alternative architectures for lightweight space telescopes using parameterized models. 2006 IEEE Aerospace Conference, Big Sky, Montana, March 4-11, 2006.

[78] Scott A. Uebelhart. Non-Deterministic Design and Analysis of Parameterized Optical Structures during Conceptual Design. PhD thesis, MIT, 2006.

[79] Scott A. Uebelhart, Lucy E. Cohan, and David W. Miller. Design exploration for a modular optical space telescope architecture using parameterized integrated models. 47th AIAA/ASME/ASCE/AHS/ASC Structures, Structural Dynamics and Materials Conference, Newport, Rhode Island, May 1-4, 2006. AIAA-2006-2083.

[80] Scott A. Uebelhart, David W. Miller, and Carl Blaurock. Uncertainty characterization in integrated modeling. In 46th AIAA/ASME/ASCE/AHS/ASC Structures, Structural Dynamics, and Materials Conference, 2005. AIAA Paper 2005-2142.

[81] Karl Wandner and Hans Kärcher. The pointing control system of SOFIA. In SPIE Astronomical Telescopes and Instrumentation, 2000.

[82] N.E. Wiberg and X.D. Li. A post-processed error estimate and an adaptive procedure for the semidiscrete finite element method in dynamic analysis. International Journal for Numerical Methods in Engineering, 37(21):3585-3603, 1994.

[83] K. Willcox and J. Peraire. Balanced model reduction via the proper orthogonal decomposition. AIAA Journal, 40(11):2323-2330, 2002.

[84] P. Zadeh and V. Toropov. Multi-fidelity multidisciplinary design optimization based on collaborative optimzation framework. In 9th AIAA/ISSMO Symposium on Multidisciplinary Analysis and Optimization, 2002. 